\title{
UICN
}

\section{Mallette pédagogique}

Pour effectuer la mission de coaching d'amélioration de la gestion des aires protégées et le développement du système d'information de l'observatoire des aires protégées et de la biodiversité du programme BIOPAMA (COMIT)

Carlo Paolini, Domoina Rakotobe et Donald Jomha Djossi

Version 1.2015

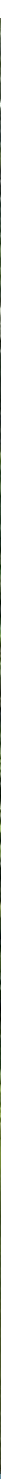

BIOPAMA (PROGRAMME POUR LA BIODIVERSITÉ ET LA GESTION DES AIRES PROTÉGÉES) - AFRIQUE CENTRALE ET DE L'OUEST

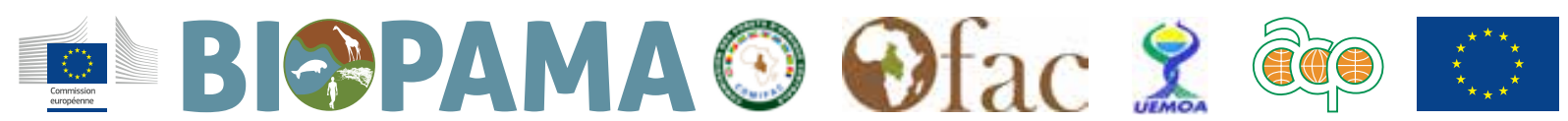


BIOPAMA

\section{À propos du BIOPAMA}

Le Programme pour la biodiversité et la gestion des aires protégées (BIOPAMA) cherche à lutter contre les menaces à la biodiversité dans les pays d'Afrique, Carailbes et Pacifique (ACP), tout en réduisant la pauvreté dans les communautés dans et autour des aires protégées. II est une initiative du Groupe ACP

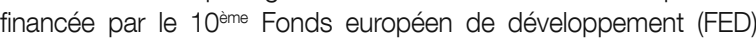
de l'Union européenne (UE). Le BIOPAMA associe l'amélioration de la disponibilité des données au renforcement des capacités, afin d'améliorer la gestion des aires protégées. II comprend deux composantes principales : I'une concerne les aires protégées et est mise en œuvre conjointement par l'Union internationale pour la conservation de la nature (UICN) et le Centre commun de recherche (CCR) de la Commission européenne, et l'autre l'Accès et le partage des avantages (APA), mis en œuvre par l'Initiative multi-donateurs de développement des capacités pour l'APA, gérée par l'Agence de coopération internationale allemande pour le développement (GIZ). www.biopama.org

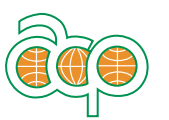

\section{À propos du Groupe des États ACP}

Le Groupe des États d'Afrique, des Carailbes et du Pacifique (ACP) est la plus grande organisation intergouvernementale transnationale de pays en développement du système international, avec 79 États membres d'Afrique, des Caraïbes et du Pacifique. Aujourd'hui, le but principal du groupe ACP est la promotion de la solidarité SudSud et la coopération Nord-Sud pour le développement durable des pays ACP et leur intégration dans l'économie mondiale. www.acp.int

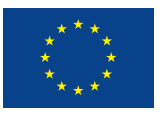

\section{À propos de l'Union européenne}

L'Union européenne est constituée de 28 États membres qui ont décidé de mettre en commun graduellement leur savoir-faire, leurs ressources et leurs destins. Durant une période d'élargissement de 50 ans, ils ont construit ensemble une zone de stabilité, de démocratie et de développement durable tout en préservant la diversité culturelle, la tolérance et les libertés individuelles. L'Union européenne est déterminée à partager ses réalisations et ses valeurs avec les pays et les peuples au-delà de ses frontières.

http://europa.eu/

\section{UICN}

\section{À propos de I'UICN}

L'UICN, l'Union internationale pour la conservation de la nature, aide le monde à trouver des solutions pragmatiques aux défis les plus pressants en matière d'environnement et de développement. L'UICN est la plus grande et la plus ancienne des organisations internationales environnementales, avec près de 1.300 gouvernements et ONG membres, et plus de 15.000 experts bénévoles répartis dans 185 pays. Le travail de l'UICN est soutenu par près de 1.000 employés répartis dans 45 bureaux, et des centaines de partenaires au sein du secteur public, des ONG, et du secteur privé dans le monde entier.

www.iucn.org

\section{À propos de la Commission européenne}

En tant que service scientifique interne de la Commission européenne, le Centre commun de recherche (Joint Research Centre - JRC) a pour mission de fournir aux politiques de l'UE un soutien scientifique et technique indépendant, fondé sur l'expérience tout au long du cycle d'élaboration des politiques. Travaillant en étroite coopération avec les directions générales chargées des politiques, le JRC relève des défis sociétaux de première importance tout en stimulant l'innovation grâce au développement de nouvelles méthodes, de nouveaux instruments et de nouvelles normes, et grâce au partage de son savoir-faire avec les États membres, la communauté scientifique et ses partenaires internationaux. https://ec.europa.eu/jrc/

\section{Ofac \\ À propos de l'Observatoire des Forêts d'Afrique Centrale}

L'OFAC est une cellule spécialisée de la COMIFAC en charge de la coordination de l'observatoire des forêts, des relations avec les antennes nationales et de la collaboration de l'ensemble des partenaires qui produisent et diffusent de l'information sur les forêts et les écosystèmes d'Afrique centrale. La cellule contribue à l'animation et à la diffusion des informations au sein du Partenariat pour les Forêts du Bassin du Congo (PFBC).

www.observatoire-comifac.net

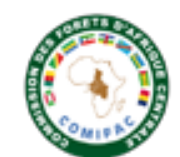

\section{À propos de la Commission des Forêts d'Afrique Centrale}

La COMIFAC est l'instance politique et technique d'orientation, de coordination, d'harmonisation et de décision en matière de conservation et de gestion durable des écosystèmes forestiers et de savanes en Afrique Centrale. Elle assure le suivi de la Déclaration de Yaoundé et veille à la mise en application des conventions internationales et des initiatives de développement forestier en Afrique Centrale.

www.comifac.org

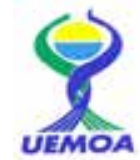

\section{À propos de l'Union économique et monétaire Ouest Africaine}

Créée le 10 janvier 1994 à Dakar, l'Union Economique et Monétaire Ouest Africaine (UEMOA) regroupe huit Etats que sont : le Bénin, le Burkina Faso, la Côte d'Ivoire, la Guinée-Bissau, le Mali, le Niger, le Sénégal et le Togo. L'UEMOA a pour objectif essentiel, l'édification, en Afrique de l'Ouest, d'un espace économique harmonisé et intégré, au sein duquel est assurée une totale liberté de circulation des personnes, des capitaux, des biens, des services, et des facteurs de production ainsi que la jouissance effective du droit d'exercice et d'établissement pour les professions libérales, de résidence pour les citoyens sur l'ensemble du territoire communautaire.

www.uemoa.int 


\section{Mallette pédagogique}

Pour effectuer la mission de coaching d'amélioration de la gestion des aires protégées et le développement du système d'information de l'observatoire des aires protégées et de la biodiversité du programme BIOPAMA (COMIT)

Carlo Paolini, Domoina Rakotobe et Donald Jomha Djossi 
La terminologie géographique employée dans cet ouvrage, de même que sa présentation, ne sont en aucune manière l'expression d'une opinion quelconque de la part de I'UICN ou des partenaires qui ont contribué à cet ouvrage sur le statut juridique ou l'autorité de quelque pays, territoire ou région que ce soit, ou sur la délimitation de ses frontières.

Les opinions exprimées dans cette publication ne reflètent pas nécessairement celles de I'UICN et ne peuvent aucunement être considérées comme reflétant le point de vue de l'Union européenne ou du Groupe des Etats ACP (Afrique, Caraïbes et Pacifique).

Le présent ouvrage a été élaboré par II'Union internationale pour la conservation de la nature (UICN) et le Centre commun de recherche de la Commission européenne (CCR-CE) dans le cadre du programme BIOPAMA (Programme pour la biodiversité et la gestion des aires protégées). Le BIOPAMA est une initiative du Groupe des Etats ACP financée par le 10ème Fonds européen de développement de l'Union européenne.

Ce document a bénéficié d'une relecture attentive, des précieux conseils et des contributions de Lawrence Baya (UICN Cameroun); Roxana Bucioaca (UICN Bruxelles); Romain Calaque (RAPAC, Cameroun); Youssouph Dieddhiou (UICN Sénégal); Kathryn Jeffery (ANPN Gabon); Albert Kembou (MINFOF, Cameroun); Rémy Ledauphin (PNUD Togo); Félicité Mangang (UICN Burkina Faso); Bora Masumbuko (UICN Burkina Faso); Elise Mazeyrac (CEDAMMMCS Gabon); Achile Mengamenya (MINFOF, Cameroun); Florence Palla (RAPAC Gabon); Sébastien Regnaut (UICN-PACO Burkina Faso); Paula Salnot (traductrice); Oumar Sadio (RAMPAO Sénégal); Carlos de Wasseige OFAC, RD Congo).

Publié par :

UICN, Gland, Suisse

Droits d'auteur :

(c) 2015 Union internationale pour la conservation de la nature et de ses ressources

La reproduction de cette publication à des fins non commerciales, notamment éducatives, est permise sans autorisation écrite préalable du [des] détenteur[s] des droits d'auteur à condition que la source soit dûment citée.

La reproduction de cette publication à des fins commerciales, notamment en vue de la vente, est interdite sans autorisation écrite préalable du [des] détenteur[s] des droits d'auteur.

Citation : $\quad$ Carlo Paolini, Domoina Rakotobe et Donald Jomha Djossi (2015). Mallette pédagogique pour effectuer la mission de coaching d'amélioration de la gestion des aires protégées et le développement du système d'information de l'observatoire des aires protégées et de la biodiversité du programme BIOPAMA (COMIT).

Gland, Suisse : UICN. 128pp.

ISBN :

$978-2-8317-1760-9$

$\mathrm{DOI}:$ http://dx.doi.org/10.2305/IUCN.CH.2015.BIOPAMA.1.fr

Photo couverture : Thomas Bacha, UICN

Photo verso : $\quad$ Thomas Bacha, UICN

Mise en page : Imre Sebestyén jr. / UNITgraphics.com

Disponible auprès de : $\quad$ UICN (Union internationale pour la conservation de la nature)

BIOPAMA, Le Programme pour la biodiversité et la gestion des aires protégées

Rue Mauverney 28

1196 Gland

Suisse

Tél +41229990000

Fax +41229990002

biopama@iucn.org

www.iucn.org/publications

Cet ouvrage est imprimé sur du papier obtenu à partir de fibre de bois provenant de forêts bien gérées, certifiées selon les normes du Forest Stewardship Council (FSC). 
PARTIE 1 : Comment parler du système d'aide à la décision $? \ldots \ldots \ldots \ldots \ldots \ldots \ldots \ldots \ldots$

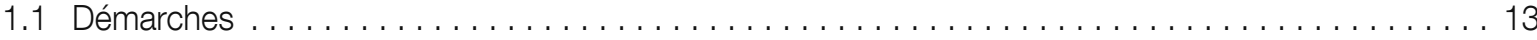

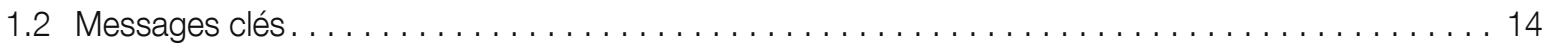

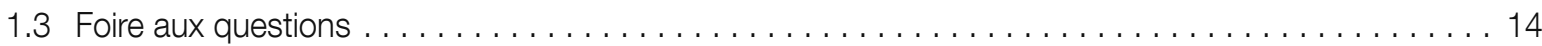

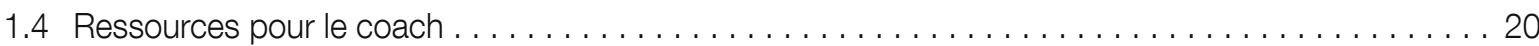

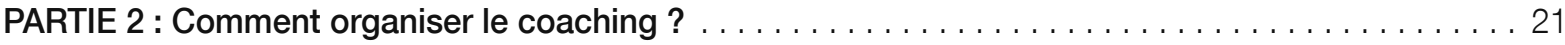

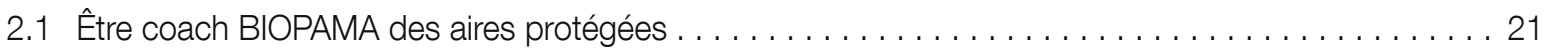

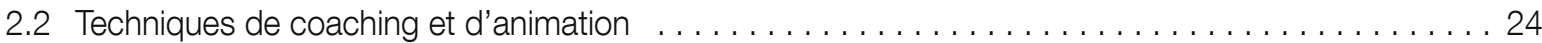

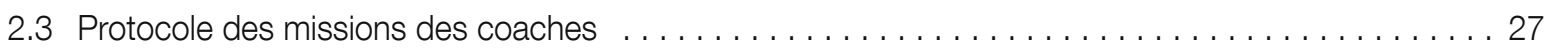

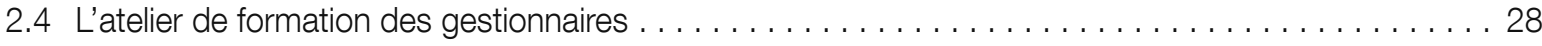

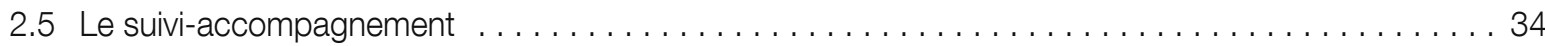

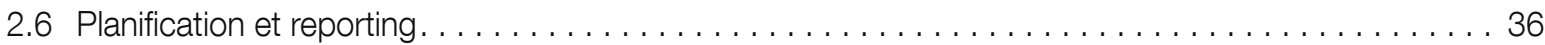

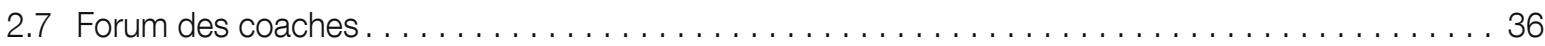

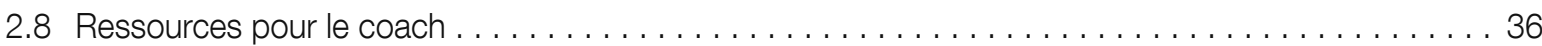

PARTIE 3 : Comment utiliser le système d'aide à la décision $? \ldots \ldots \ldots \ldots \ldots \ldots \ldots \ldots \ldots \ldots$

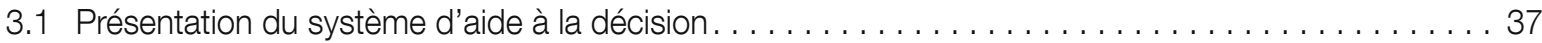

3.2 Utiliser le formulaire . . . . . . . . . . . . . . . . . . . . . . . . . . . . . . . . 54

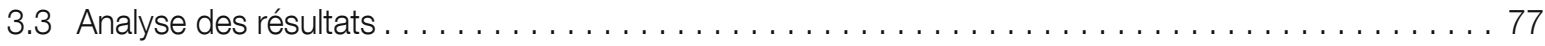

3.4 Formulation des propositions d'amélioration $\ldots \ldots \ldots \ldots \ldots \ldots \ldots \ldots \ldots \ldots \ldots \ldots \ldots$

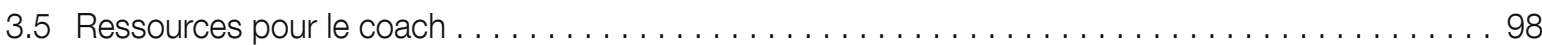

PARTIE 4 : Encodage des données dans le formulaire biopama $\ldots \ldots \ldots \ldots \ldots \ldots \ldots \ldots \ldots$

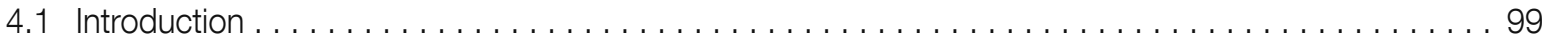

4.2 Accès au formulaire . . . . . . . . . . . . . . . . . . . . . . . . . . . . . . . . . . . . . . 100

4.3 Encodage des données.......................................... 101

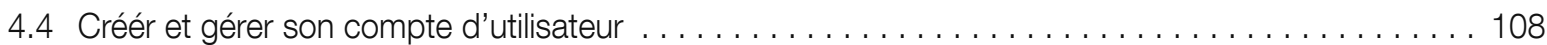

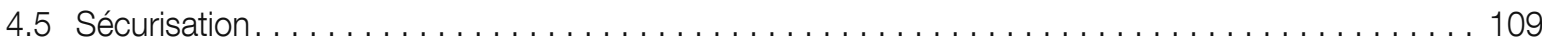

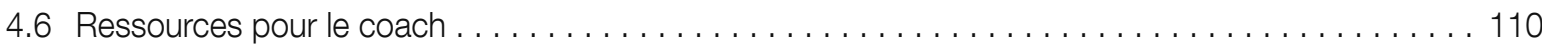

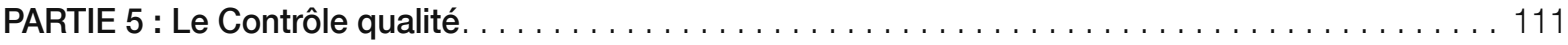

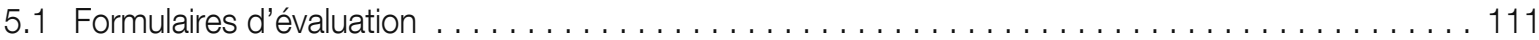

5.2 Ressources pour le coach . . . . . . . . . . . . . . . . . . . . . . . . . . . . . . . . . . . 120

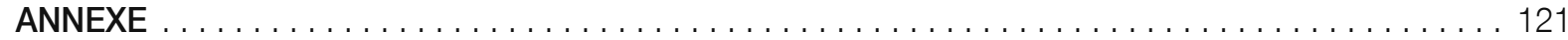




\section{LISTE DES EXERCICES}

Exercice 1 - Philosophie du coaching . . . . . . . . . . . . . . . . . . . . . . . . 26

Exercice 2 - Identification des pressions, menaces et vulnérabilités $\ldots \ldots \ldots \ldots \ldots \ldots \ldots \ldots \ldots$

Exercice 3 - Structuration schématique des menaces . . . . . . . . . . . . . . . . . . . . . . . . .44

Exercice 4 - Analyse Contrainte - Support -Pouvoir des acteurs . . . . . . . . . . . . . . . . . . . . 444

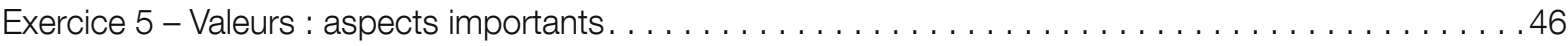

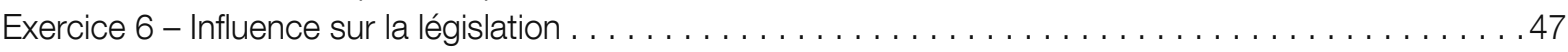

Exercice 7 - Changement du degré de classement $\ldots \ldots \ldots \ldots \ldots \ldots \ldots \ldots \ldots \ldots \ldots \ldots$

Exercice 8 - Pertinence de l'approche de gestion d'une AP . . . . . . . . . . . . . . . . . . .48

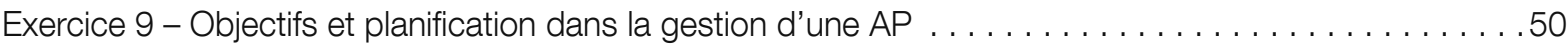

Exercice 10 - Analyse Contrainte - Support -Pouvoir des acteurs 2 . . . . . . . . . . . . . . . . . .74

Exercice 11 - Analyse du contexte de la gestion. . . . . . . . . . . . . . . . . . . . . . . . . . . . . 79

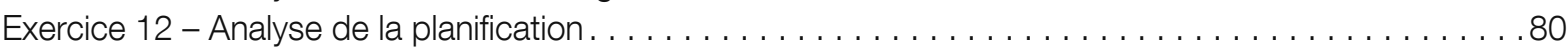

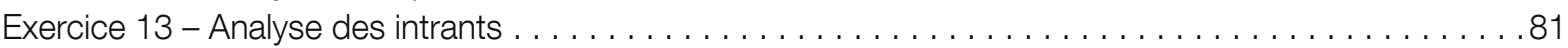

Exercice 14 - Analyse des systèmes et processus de gestion $\ldots \ldots \ldots \ldots \ldots \ldots \ldots \ldots \ldots$

Exercice 15 - Analyse de la protection . . . . . . . . . . . . . . . . . . . . . . . . . . 82

Exercice 16 - Analyse des relations avec les parties prenantes. . . . . . . . . . . . . . . . 83

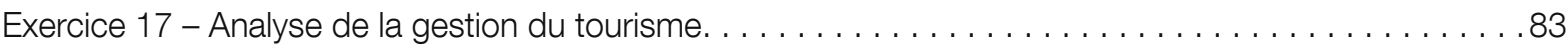

Exercice 18 - Analyse du suivi et de la recherche . . . . . . . . . . . . . . . . . . . . . . . . . . 84

Exercice 19 - Analyse de la gestion des effets du changement climatique et des services écosystémiques . 84

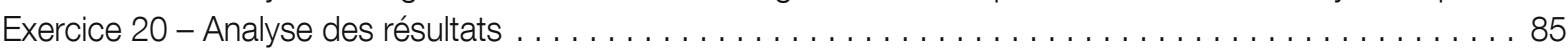

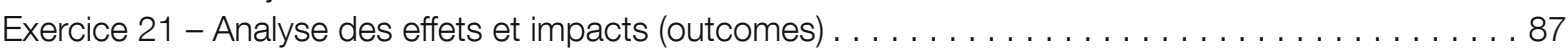

Exercice 22 - Éléments prioritaires à gérer . . . . . . . . . . . . . . . . . . . . . . . . . . . . . . . . 91

\section{LISTE DES ENCADRES}

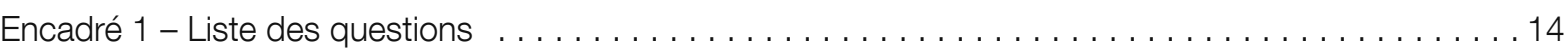

Encadré 2 - La charte du coach BIOPAMA des aires protégées . . . . . . . . . . . . . . . . . . . 22

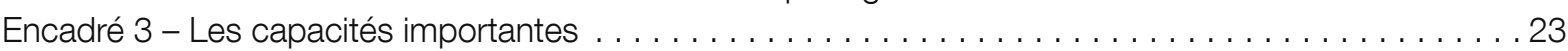

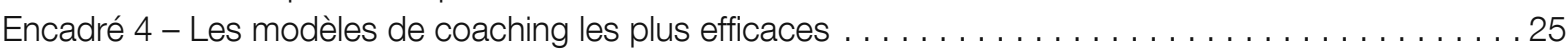

Encadré 5 - Quelques principes et techniques d'andragogie . . . . . . . . . . . . . . . . . . . . . . . 29

Encadré 6 - Liste de contrôle des tâches et calendrier de préparation . . . . . . . . . . . . . . . . . . . . . 31

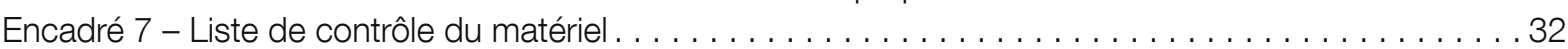

Encadré 8 - Modèle de programme de l'atelier national . . . . . . . . . . . . . . . . . . . . . 33

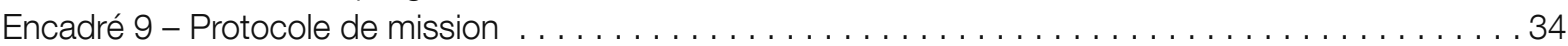

Encadré 10 - Liste de contrôle sur la préparation logistique . . . . . . . . . . . . . . . . . . . . . . . . . 35

Encadré 11 - La gestion adaptative, une approche structurée pour la prise de décision . . . . . . . . . . 37

Encadré 12 - Les éléments à mesurer . . . . . . . . . . . . . . . . . . . . . . . . . . . . . . . . . . . . 49

Encadré 13 - Enseignements tirés à la suite du remplissage du Formulaire lors des phases test . . . . . 64

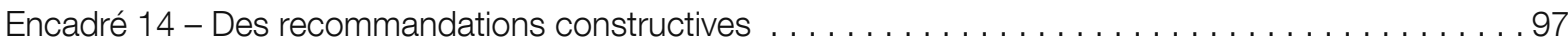

\section{LISTE DES TABLEAUX}

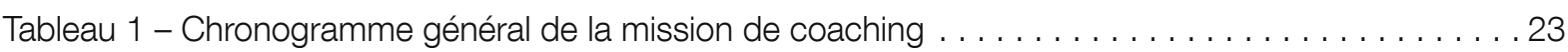

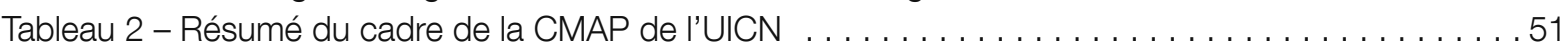

Tableau 3 - Objectifs et critères d'évaluation du cadre de la CMAP de l'UICN . . . . . . . . . . . . . . . 52

Tableau 4 - Exemple de gestion de la population des éléphants dans un parc national . . . . . . . . . 53 


\section{LISTE DES FIGURES}

Schéma 1 - Liens entre les services fournis par les écosystèmes et le bien-être humain. . . . . . . . 42

Schéma 2 - Graphique « Contrainte - Support - Pouvoir des acteurs » sur les interactions des

principaux acteurs du contexte de l'intervention avec l'aire protégée . . . . . . . . . . . .44

Schéma 3 - Environnement managérial d'une aire protégée (d'après Cusworth and Francks, 1993

in Hockings, et al.2008) . . . . . . . . . . . . . . . . . . . . . . . . . . . . 45

Schéma 4 - Liaison fonctionnelle entre les éléments du contexte d'intervention et l'efficacité de gestion .46

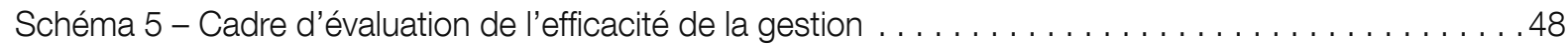

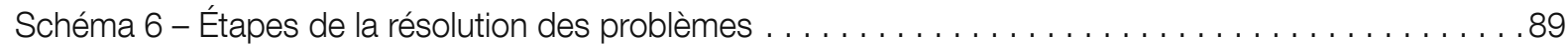

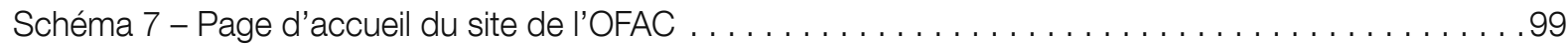

Schéma 8 - Page de connexion en ligne. . . . . . . . . . . . . . . . . . . . . . . . . . . 100

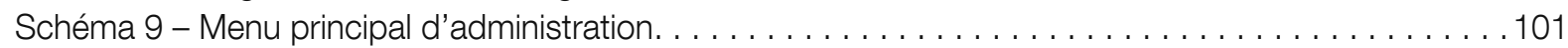

Schéma 10 - Extraction du fichier ZIP . . . . . . . . . . . . . . . . . . . . . . . . . . . . . 101

Schéma 11 - Choix de l'emplacement du dossier . . . . . . . . . . . . . . . . . . . . . . . . . . . . 102

Schéma 12 - Création du raccourci sur le bureau. . . . . . . . . . . . . . . . . . . . . . . . . . . . . 102

Schéma 13 - Page de connexion hors ligne. . . . . . . . . . . . . . . . . . . . . . . . . . 103

Schéma 16 - Liste de formulaires créés par pays . . . . . . . . . . . . . . . . . . . . . . . . . . 104

Schéma 14 - Menu principal d'administration. . . . . . . . . . . . . . . . . . . . . . . . . . . . . . . . . . . . . . . . 104

Schéma 15 - Sélection de pays . . . . . . . . . . . . . . . . . . . . . . . . . . . . . . . . . . . . . . . . 104

Schéma 17 - Création d'un formulaire . . . . . . . . . . . . . . . . . . . . . . . . . . . . 105

Schéma 19 - Constitution d'un module . . . . . . . . . . . . . . . . . . . . . . . . . . . . . . . . 106

Schéma 18 - Barre de sélection de pages . . . . . . . . . . . . . . . . . . . . . . . . . . 106

Schéma 20 - Insertion d'éléments . . . . . . . . . . . . . . . . . . . . . . . . . . . . . . . . . . . . 107

Schéma 21 - Boite de dialogue d'invite de recherche. . . . . . . . . . . . . . . . . . . . . . . . . . 107

Schéma 22 - Les menus accordéon . . . . . . . . . . . . . . . . . . . . . . . . . . . . . 108

Schéma 23 - Réinitialisation de barème . . . . . . . . . . . . . . . . . . . . . . . . . 108

Schéma 24 - Page de connexion (Initier la création d'un compte). . . . . . . . . . . . . . . . . . . . . 109

Schéma 25 - Création de compte d'utilisateur . . . . . . . . . . . . . . . . . . . . . . . . . . . . . . . . 109

Schéma 26 - Menu d'administration (Modifier son profil) . . . . . . . . . . . . . . . . . . . . . . . . 110

Schéma 27 - Modification des données de profil d'utilisateur . . . . . . . . . . . . . . . . 110 


\begin{tabular}{|c|c|}
\hline $\mathrm{ACP}$ & Afrique, Caraïbes et Pacifique \\
\hline AP & Aire protégée \\
\hline BIOPAMA & Programme pour la biodiversité et la gestion des aires protégées \\
\hline CARPE & Programme régional de l'Afrique centrale pour l'environnement \\
\hline CCR & Centre commun de recherche (UE) \\
\hline CMAP & Commission mondiale des aires protégées \\
\hline COMlT & $\begin{array}{l}\text { Mallette pédagogique pour effectuer la mission de coaching d'amélioration de la gestion } \\
\text { des aires protégées et le développement du système d'information de l'Observatoire des } \\
\text { aires protégées et de la biodiversité du programme BIOPAMA / Coach Mission Observatory } \\
\text { Information Toolkit }\end{array}$ \\
\hline DOPA & Observatoire numérique pour les aires protégées / Digital Observatory for Protected Areas \\
\hline DSS & Support d'aide à la décision / Decision Support System \\
\hline EoH & Mise en valeur de notre patrimoine - UNESCO / Enhancing Our Heritage - UNESCO \\
\hline GIZ & $\begin{array}{l}\text { Agence de coopération internationale allemande pour le développement / } \\
\text { Deutsche Gesellschaft für Internationale Zusammenarbeit }\end{array}$ \\
\hline IBA & Zones importantes pour les oiseaux / Important Bird Areas \\
\hline $\mathrm{ICCN}$ & Institut congolais pour la conservation de la nature \\
\hline LAB & Lutte anti-braconnage \\
\hline MAB & Programme L'homme et la biosphère/ Man and Biosphere \\
\hline MEIT & Outil de suivi de l'efficacité de la gestion / Management Effectiveness Tracking Tool \\
\hline OBAPAC & Observatoire des aires protégées du bassin du Congo \\
\hline OFAC & Observatoire des forêts d'Afrique centrale \\
\hline PAME & Efficacité de la gestion des aires protégées / Protected Area Management Effectiveness \\
\hline PAMETT & $\begin{array}{l}\text { Outil de suivi de l'efficacité de la gestion des aires protégées / Protected Area Management } \\
\text { Effectiveness Tracking Tool }\end{array}$ \\
\hline RAMPAO & Réseau régional d'aires marines protégées en Afrique de l'Ouest \\
\hline RAPAC & Réseau des aires protégées d'Afrique centrale \\
\hline RAPPAM & $\begin{array}{l}\text { Évaluation rapide \& priorisation de la gestion des aires protégées / Rapid Assessment \& } \\
\text { Prioritization of Protected Area Management }\end{array}$ \\
\hline RRIS & Système régional d'information de référence / Regional Reference Information System \\
\hline UICN & Union internationale pour la conservation de la nature \\
\hline PACO & Programme Afrique centrale et occidentale \\
\hline WWF & Fonds mondial pour la nature / World Wild Fund for Nature \\
\hline
\end{tabular}




\section{INTRODUCTION}

Le Programme pour la biodiversité et la gestion des aires protégées - Biodiversity and Protected Areas Management (BIOPAMA) - cherche à lutter contre les menaces qui pèsent sur la biodiversité dans les pays d'Afrique, Caraïbes et Pacifique (ACP), touten réduisant la pauvreté dans les communautés aux alentours et dans les aires protégées. Plus précisément, le Programme veut améliorer l'accès aux connaissances scientifiques disponibles pour consolider le travail des institutions et réseaux existants, et renforcer les capacités des décideurs et administrateurs dans la conservation de la biodiversité, la gestion des aires protégées, et l'accès et le partage des avantages.

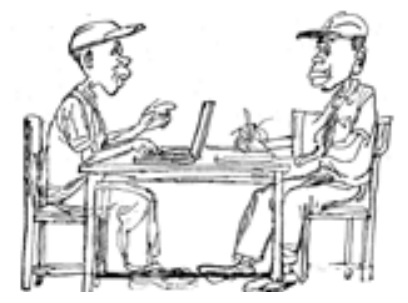

La stratégie de développement des capacités du programme BIOPAMA de l'Afrique centrale et occidentale vise à encourager les gestionnaires et administrateurs des aires protégées (AP) à utiliser le Système régional d'information, ou Observatoire régional sur la biodiversité et les aires protégées. Elle préconise pour l'année 20152016 la formation de formateurs-accompagnateurs, dénommés "Coaches BIOPAMA des aires protégées " (ou " coaches " dans le présent document) pour inciter prioritairement les gestionnaires des AP et ensuite les responsables au niveau national et régional à comprendre et à utiliser le Système d'information d'aide à la décision dans les processus d'analyse, de planification, de suivi, et d'évaluation de la gestion et de la gouvernance de la biodiversité et des AP.

Le présent document (la Mallette pédagogique pour effectuer la mission de coaching et développer le système d'information de l'Observatoire des aires protégées et de labiodiversitédu programmeBIOPAMA (COMIT)) est un support didactique destiné à aider les coaches à accomplir leur mission de formation/ accompagnement. La COMIT comprend le présent document imprimé ainsi qu'une clé USB contenant la version OFFLINE (la version ONLINE est disponible sur le site OFAC, http://www.observatoire-comifac.net/ admin/home.php) du Formulaire du système d'aide à la décision et des fichiers électroniques de support pour son utilisation. C'est un document de base, flexible, que le coach pourra adapter aux différentes situations et typologies de conservation et de gestion des ressources naturelles.

La COMIT peut être utilisée par une gamme plus large de bénéficiaires, avec des degrés variés d'adéquation par rapport aux objectifs de l'utilisateur, entre autres : les responsables de la planification et du suiviévaluation d'une AP ou des réseaux d'AP peuvent se former à l'utilisation du système d'aide à la décision grâce à la COMIT ; les formateurs professionnels ou en milieu universitaire dans les domaines relatifs à la gestion des aires protégées y trouveront des éléments pertinents sur l'évaluation et l'amélioration de l'efficacité de la gestion à intégrer dans leur programme ; et enfin, tous conservateurs désireux d'utiliser le système d'aide à la décision.

La COMIT est divisée en quatre parties distinctes :

- PARTIE 1: Comment parler du système d'aide à la décision?

- PARTIE 2 : Comment organiser le coaching ?

- PARTIE 3 : Comment utiliser le système d'aide à la décision?

- PARTIE 4 : Contrôle - qualité

Chaque partie comprend :

- Un texte explicatif ;

- Des encadrés permettant une lecture facile des points importants ;

- Une section "Ressources pour le coach" contenant une liste de documents et de sites web pour approfondir la section; et des contacts en cas de difficultés ;

- Des astuces pour le coach respectant la codification suivante :

La COMIT contient aussi des exercices pratiques basés sur des cas réels d'utilisation du formulaire d'évaluation de l'efficacité de la gestion!

\section{Codification/symbole}

(国): Notes pour le coach donnant les principes fondamentaux sur un thème précis

: Astuces pour accélérer, servir de repère, attirer l'attention dans le coaching

‡ : Exemple de questions à poser à un gestionnaire de l'AP

: Techniques de coaching suggérées 


\begin{tabular}{|c|c|}
\hline Dossier & \\
\hline $\begin{array}{l}\text { 0-Documents } \\
\text { de base }\end{array}$ & $\begin{array}{l}\text { 0-1Biopama_fact_sheet_fr_version } \\
\text { 0-2Biopama_presentation_ppt_fr__ } \\
\text { 0-3 Systeme d'information DSS } \\
\text { 0-4 C-EVAL1 } \\
\text { 0-5 C-EVAL2 } \\
\text { 0-6 C-EVAL3 } \\
\text { 0-7 Rapport Mission Parc } \\
\text { 0-8 Liste Participants Annexe 1 } \\
\text { 0-8 Rapport financier mission \& budget }\end{array}$ \\
\hline $\begin{array}{l}\text { 1-Gestion et } \\
\text { gouvernance } \\
\text { des AP }\end{array}$ & $\begin{array}{l}\text { Catégories UICN de gestion des AP } \\
\text { Lignes directrices pour la législation des AP } \\
\text { No.08 Sustainable tourism in PAs } \\
\text { No.10 Lignes directrices pour la planification de la gestion des AP } \\
\text { No.15 ldentification et analyse des lacunes des Zones clés pour la biodiversité } \\
\text { No.16 Sites naturels sacrés } \\
\text { No.18 Restauration écologique des AP } \\
\text { No.20 Gouvernance des AP } \\
\text { Triplet (2009) Manuel de gestion des AP francophones }\end{array}$ \\
\hline $\begin{array}{l}\text { 2-Efficacité de } \\
\text { la gestion }\end{array}$ & $\begin{array}{l}\text { 2-1 Coad et al (2013) Progress towards the CDB protected area management } \\
\text { effectiveness targets } \\
\text { 2-2 Hockings et al (2008) Evaluating effectiveness A framework for assessing } \\
\text { management effectiveness of protected areas } \\
\text { 2-3 Hockings et al (2008) Évaluation de l'efficacité Un cadre pour l'évaluation de l'efficacité } \\
\text { de la gestion des aires protégées } \\
\text { 2-4 Leverington et al (2008) Management effectiveness evaluation in protected areas - a } \\
\text { global study } \\
\text { 2-5 Leverington et al (2008) Management effectiveness evaluation in protected areas - a } \\
\text { global study Overview of approaches and methodologies } \\
\text { 2-6 Leverington et al (2010) Management effectiveness evaluation in protected areas - a } \\
\text { global study. 2nd Edition. } \\
\text { 2-7 Leverington et al (2010) A global analysis of protected areas management } \\
\text { effectiveness } \\
\text { 2-8 RAMPAO Évaluation de l'efficacité de la gestion des AMP du réseau des aires marines } \\
\text { protégées en Afrique de l'Ouest - RAMPAO }\end{array}$ \\
\hline $\begin{array}{l}\text { 3-Formulaire } \\
\text { hors ligne }\end{array}$ & Formulaire \\
\hline $\begin{array}{l}\text { 4-Coaching et } \\
\text { formation }\end{array}$ & $\begin{array}{l}\text { 4-1 AIR (2005) Conceptual overview : coaching in the professional development impact } \\
\text { study } \\
\text { 4-2 No.17 Protected Area staff training } \\
\text { 4-3 Meriaux \& Staub Guide_formation }\end{array}$ \\
\hline $\begin{array}{l}\text { 5-Conservation } \\
\text { en général }\end{array}$ & $\begin{array}{l}\text { 5-1Guidelines for Application of IUCN Red List Criteria } \\
\text { 5-2 IUCN Red List Brochure } \\
\text { 5-3 IUCN Red List Categories and Criteria } \\
\text { 5-4 IUCN Red List of Ecosystems } \\
\text { 5-5 Triplet_2015_Dictionnaire de la conservation } \\
\text { 5-6 UE Bien au-delà des éléphants Vol } 4 \text { AC } \\
\text { 5-7 UE Bien au-delà des éléphants Vol } 5 \text { AO } \\
\text { 5-8 WHO Ecosystem and human wb }\end{array}$ \\
\hline
\end{tabular}

NB: Des documents supplémentaires sur la formation des coaches à La Tapoa ont complété la clé 


\title{
Le Formulaire BIOPAMA (IMET, Integrated Management Effectiveness Tool ${ }^{1}$ )
}

\author{
Un outil intégré de planification, suivi et évaluation des aires protégées²
}

\section{Introduction}

Pour contribuer à l'amélioration de la gestion des aires protégées et l'atteinte des objectifs de conservation, le programme BIOPAMA propose un outil intégré en support de la planification, de suivi et de l'évaluation des aires protégées. Le formulaire du BIOPAMA dénommé IMET (outil intégré sur l'efficacité de gestion/Integrated Management Effectiveness Tool) favorise l'adoption d'une approche proactive axée sur les résultats, grâce à des outils qui peuvent décrire et quantifier l'environnement et son évolution.

En organisant les informations disponibles selon des modèles prédéfinis pour élaborer des systèmes d'aide à la décision ou DSS (Support d'aide à la décision/ Decision Support System), il est possible de définir des niveaux de référence, de faciliter la planification, d'analyser l'état de conservation et de concentrer les évaluations de l'efficacité de la gestion sur les objectifs de conservation.

Le formulaire IMET a ainsi été conçu et développé afin de soutenir directement les gestionnaires, sur le terrain ou au niveau central (agences nationales des aires protégées), à améliorer l'efficacité de la gestion des aires protégées et, plus généralement, la conservation de la biodiversité.

\section{BIOPAMA IMET}

Le formulaire IMET - conçu à l'origine pour les pays d'Afrique centrale et de l'ouest, mais utilisable pour toute aire protégée, aussi bien terrestre que marine est constitué de trois modules :

1. l'évaluation de l'état du contexte de l'intervention;

2. l'évaluation de l'efficacité de la gestion sur la base des références fournies par I'UICN (M. Hockings, F. Leverington et al.) ;

3. la visualisation des éléments et des analyses produites comme système d'aide à la décision.

\footnotetext{
1 Les Formulaires BIOPAMA sont désormais identifies comme ("IMET", Integrated Management Effectiveness Tool). Toutefois, cette dénomination ayant été définie successivement à la finalisation de ce rapport, dans le document la dénomination IMET n'est pas utilisée.

2 Auteurs: Carlo Paolini (consultant CCR) et Paolo Roggeri (CCR)
}

De ce fait, le formulaire IMET ne constitue pas un nouvel outil d'évaluation. Toutefois, la structuration des informations, la quantification des effets et impacts visés et la possibilité de visualiser la contribution relative de chacun d'entre eux à l'efficacité de gestion, fournissent aux gestionnaires tous les éléments leur permettant d'analyser la situation actuelle, d'identifier les points forts et les faiblesses et de définir les améliorations nécessaires pour atteindre les objectifs.

Le traitement statistique sous-tend et soutient les modalités de collecte et traitement des informations, l'utilisation des indicateurs simples et composés, le système d'évaluation, visualisation et interprétation de données. Le formulaire IMET conduit les parties prenantes à travers les différents éléments du puzzle de la gestion des aires protégées vers l'adoption d'une approche proactive aux efforts de conservation. II permet :

- d'identifier les éléments clé et prioritaires de la gestion afin d'adopter une démarche qui permette d'évoluer de la condition de conservation actuelle à celle favorable/ souhaitée ;

- d'orienter les interventions vers des objectifs ciblés et clairement identifiés de manière qualitative ou quantitative ;

- d'adopter une approche proactive dans les efforts de conservation.

\section{Module "Contexte d'intervention »}

Le module Contexte d'intervention apporte des informations détaillées :

- d'ordre général ;

- sur les superficies, limites et indice de forme, niveau de contrôle de l'aire protégée ;

- sur les ressources à disposition de la gestion des aires protégées ;

- sur les espèces, les habitats, la couverture des sols, les changements dans la couverture des sols, etc., suivant en partie les schémas d'information de l'Observatoire numérique DOPA, du CCR-EC ; 
- $\quad$ sur les menaces sur une base « revisitée » du calculateur des menaces ${ }^{3}$;

- $\quad$ sur les effets du changement climatique ;

- $\quad$ sur les services écosystémiques.

\section{Module "Efficacité de la gestion 》}

Le module "Efficacité de la gestion » prend ses origines de plusieurs sources:

- METT (Management Effectiveness Tracking Tool) ;

- $\quad$ EoH (Enhancing Our Heritage);

- RAPPAM (Rapid Assessment and Prioritization of Protected Areas Management) ;

- Global study, Secondedition-2010Management effectiveness évaluation in protected areas pour les indicateurs de synthèse et principaux identifiés par l'étude.

Ce module est un élément essentiel du puzzle du système de gestion des aires protégées.

Il adopte les indicateurs principaux et de synthèse proposés par l'analyse du document Global Study et les éléments du METT réorganisés suivant les différents éléments du cycle de gestion des aires protégées. Des éléments spécifiques et des indicateurs supplémentaires ont été rajoutés pour le niveau de gestion et de contrôle des valeurs et des éléments d'importance, les effets du changement climatique et les services écosystémiques à prendre en compte dans la gestion des aires protégées.

\section{Visualisation}

Les informations récoltées, organisées et élaborées statistiquement sont déployées à l'aide d'instruments de visualisation pour faciliter leur analyse et interprétation. L'utilisation régulière du formulaire IMET permet de suivre les évolutions des éléments clé dans le temps et, de ce fait, d'assurer l'adaptabilité de la gestion. L'objectif du système DSS est de rendre les facteurs et les phénomènes liés au processus de gestion plus compréhensibles, intégrés et facilitant la prise de décision aux différents niveaux de la gouvernance environnementale.

3 Le concept de Threat calculator (Calculateur de menaces) a été développé par un groupe de travail mis en place par NatureServe en 2004 (http://www.natureserve.org/conservation-tools/conservationrank-calculator). La classification des menaces a été adoptée par la CMAP de l'UICN, Salafsky et al. en 2008 (http://www.iucnredlist.org/ technicaldocuments/classification-schemes/threats-classification-scheme).

\section{Objectifs et approche}

Le formulaire IMET facilite l'identification des potentiels objectifs de l'aire protégée et des lignes directrices conséquentes pour améliorer l'efficacité de la gestion.

Les gestionnaires sont invités à identifier les éléments clé du contexte de gestion à partir d'un plus large examen du contexte d'intervention (contexte élargi) $^{4}$. Le formulaire IMET suggère le suivi de la tendance des éléments clé de la gestion par la définition d'un niveau de référence (baseline) et de la condition ou l'état de conservation souhaités. Le formulaire prévoit une matrice pour la détermination des objectifs, des indicateurs ${ }^{5}$ et des valeurs de référence (benchmarks) pour permettre d'assurer le suivi dans la démarche de gestion.

Le but, progressivement dans le temps, est de : i) disposer des informations nécessaires à la gestion ; ii) faciliter la mise en place d'interventions en faveur de la conservation fortement ciblées sur les résultats (outputs) et sur les effets/impacts (outcomes) attendus, identifiés aussi bien de manière qualitative que quantitative (approche proactive) ; et iii) favoriser ainsi la réflexion pour évoluer de l'état actuel d'intervention vers un état favorable de conservation et de maintien des services écosystémiques.

La formulation des objectifs et des stratégies de conservation impose le paramétrage du formulaire IMET par rapport aux aspects clé de la gestion de chaque aire protégée.

\section{Informations disponibles, niveaux d'intervention et flux d'informations}

La détermination de l'état du contexte d'intervention et l'évaluation de l'efficacité de la gestion, orientées vers l'identification des priorités de gestion et de gouvernance des aires protégées, reposent sur la collecte et l'organisation d'informations ciblées.

Les informations utilisées par les utilisateurs du formulaire IMET doivent être classifiées selon un degré de fiabilité, Élevé, Moyen et Faible, selon des

4 Dans l'analyse du contexte de gestion il est suggéré de ne retenir qu'un nombre réduit d'indicateurs pour les aspects plus importants de la gestion de l'aire protégée.

5 Suivant les indications du Biodiversity Indicator Partnership (BIP), http://www.bipindicators.net/ 
critères déterminés. La proposition d'utiliser des informations de moyenne ou faible fiabilité dans le processus de prise de décision est déterminée par les impératifs d'action et la nécessité d'identifier les connaissances prioritaires à améliorer à l'avenir.

Puisque le système s'appuie sur un modèle standardisé de collecte et sur l'analyse statistique des informations, il est possible d'établir des liaisons fonctionnelles entre les niveaux de gestion "Site de conservation - Landscape - Ecosystème - Réseau national - Réseau régional ».

Par le même principe il est possible d'établir des flux d'informations top-down et bottomup en aide à la prise de décision entre, d'une part, les différents niveaux de gestion et de gouvernance environnementale, et, d'autre part, les Observatoires BIOPAMA. Ce double échange d'information assure un renforcement mutuel et adaptatif, aussi bien dans l'amélioration des connaissances et que dans la définition des stratégies et des plans de gestion.

\section{Conclusions}

Le formulaire IMET vise à améliorer l'utilisation et l'appropriation des outils de planification-suiviévaluation au niveau des sites de conservation et des administrations au niveau central par l'orientation des informations à la prise de décisions avisée. Son utilisation est un objectif réaliste, puisque l'outil répond directement aux besoins des parties prenantes, concrètement, dans leur travail quotidien. Tous les tests effectués jusqu'à présent (Gabon1, RDC, Gabon2, Bolivie1, Bolivie2, COMIT), prouvent que I'utilisation du formulaire IMET n'a jamais été perçue comme un exercice supplémentaire inutile pour le personnel des sites de conservation et des agences nationales.

Malgré l'envergure de l'exercice, le remplissage du formulaire IMET ne présente pas un niveau de complexité particulière. Par contre, les choix des éléments clé de conservations et des éléments de suivi (indicateurs) relatifs, les analyses et la formulation des propositions de gestion, requièrent des capacités appropriées. C'est pour cela que I'adoption du formulaire IMET impose un changement dans les approches de formation avec la création des coaches dans le domaine de la conservation et la conception de cette boîte à outils nommée COMIT (Coach Mission Observatory Information Toolkit) pour l'utilisation du formulaire IMET.
Les coaches sont des opérateurs experts du formulaire IMET pour la diffusion et l'appropriation de l'outil et du processus de planification-suiviévaluation auprès des gestionnaires et l'amélioration de la gestion des sites de conservation. En même temps les coaches doivent être des opérateurs du système d'information "multi-segment " pour assurer l'indispensable liaison fonctionnelle à l'intérieur du réseau de conservation et pour pouvoir représenter les points focaux des observatoires digitaux des aires protégées (Observatoires BIOPAMA).

Dans le processus Planification - Suivi - Évaluation et dans le système d'analyse et de soutien à la prise de décision, le formulaire IMET :

- constitue une monographie synthétique des multiples aspects de l'aire protégée en examen ;

- représente une synthèse très opérationnelle de la planification de gestion (voir plan de gestion, plan financier, plan de travail) ;

- constitue la base de référence pour la gestion en l'absence d'instruments de programmation à moyen et long terme ;

- assure la cohérence entre les instruments de programmation à long terme et annuels et la continuité entre les instruments de planification annuelle ;

- constitue un système simplifié de suivi des interventions et des valeurs clé de conservation ;

- constitue un système d'autoévaluation caractérisé et gérable par les sites de conservation et les instances de coordination centrale.

En bref, le formulaire IMET consolide la partie conceptuelle de base des multiples systèmes de suivi et, de ce fait, vise à faciliter les gestionnaires dans le choix des parcours à adopter pour améliorer sur le terrain la gestion et la gouvernance de la biodiversité et des services écosystémiques des aires protégées. 
COMIT 


\section{PARTIE 1}

\section{Comment parler du système d'aide à la décision ?}

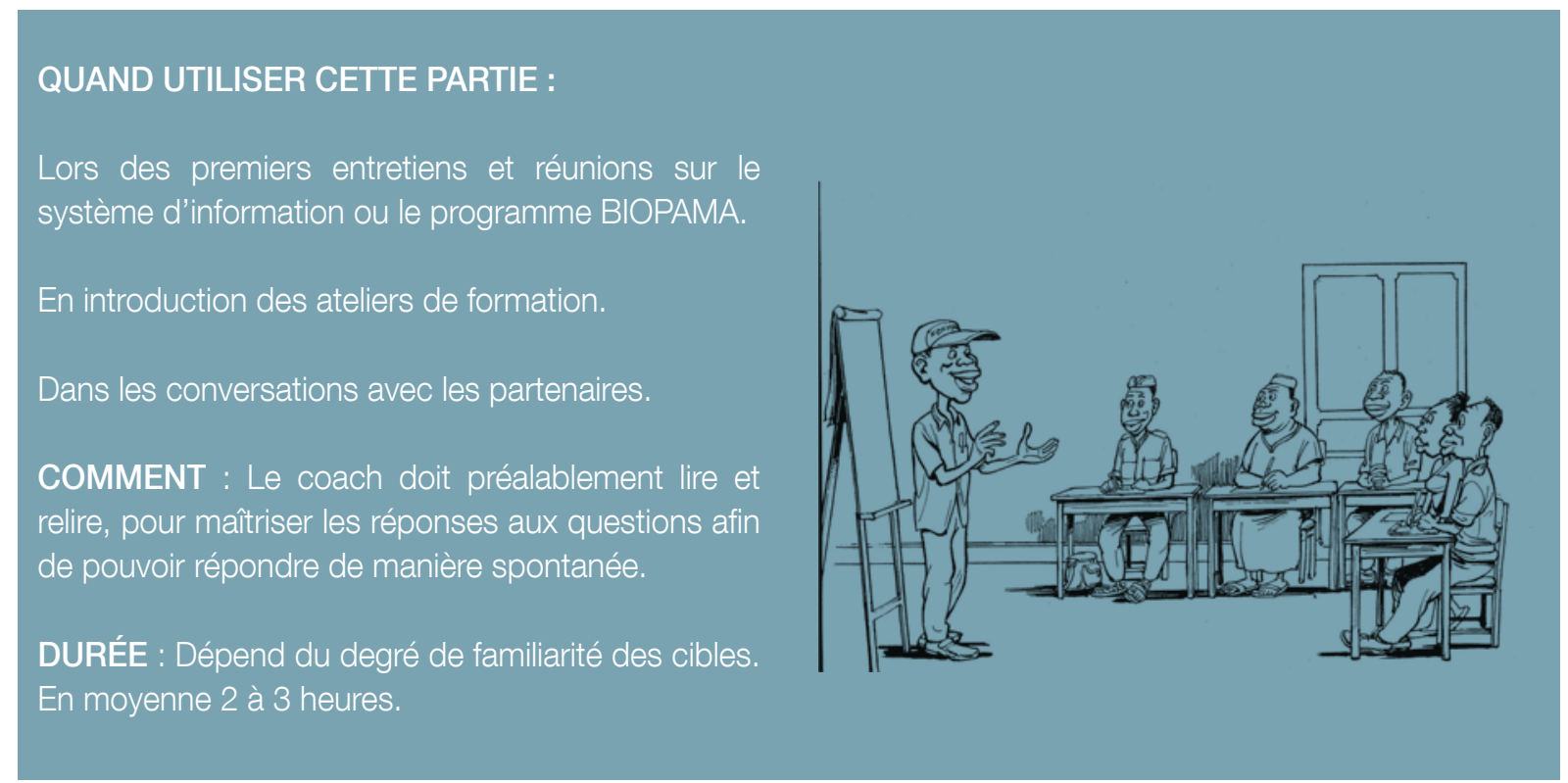

\subsection{Démarches}

Le programme BIOPAMA Afrique centrale et occidentale a pour objectif de soutenir les institutions et les individus gestionnaires des aires protégées, d'où une démarche considérant à la fois le cadre et les besoins institutionnels, ainsi qu'un accompagnement personnalisé au niveau de chaque équipe de gestion.

\section{Étape1 : Prise de contact}

Cette étape vise à introduire le coach et le système d'aide à la décision du programme BIOPAMA auprès des institutions nationales.

Habilité par le Programme des aires protégées pour l'Afrique centrale et occidentale de I'UICN (UICN-PACO) et soutenu par ses partenaires sous-régionaux et nationaux, le coach sera mis en contact avec l'institution nationale en charge de la gestion des aires protégées. II s'agit d'effectuer une visite de courtoisie ou une réunion introductive pour présenter le programme BIOPAMA, l'Observatoire régional et le système d'aide à la décision ainsi que les principales étapes de renforcement des capacités et de collecte de données. Cette étape assurera une pleine appropriation de l'approche et du projet par l'institution, et identifiera les interlocuteurs permanents du coach pour le projet.

Si le coach fait déjà partie de l'institution, cette étape sera orientée vers une discussion interne de la collaboration avec le programme BIOPAMA auprès de ses supérieurs hiérarchiques (étape 2), notamment pour que tout le travail de coaching puisse se faire jusqu'à la fin du processus même s'il y a un changement brutal du poste du coach dans l'institution.

Étape 2 : Présentation et discussion de la démarche (au moins deux mois avant la date prévue de formation nationale des acteurs de la gestion et gouvernance des AP).

Cette étape vise à clarifier le partenariat et organiser les étapes par une réunion de travail comprenant les responsables impliqués dans la planification/ les orientations stratégiques/les prises de décision au sein des institutions nationales en charge de la gestion des aires protégées. Le coach travaillera 
étroitement avec les interlocuteurs préalablement identifiés lors de l'étape 1.

\section{Agenda de réunion}

- Échanges avec les administrateurs et acteurs de la conservation et de la gestion des ressources naturelles sur le programme général ;

- Définition du choix des aires protégées avec les institutions en charge ;

- Décision des noms des participants à la formation nationale des gestionnaires des AP ;

- Arrêt de la date de l'atelier de formation ;

- Partage du programme-type d'accompagnement des gestionnaires des sites test et de la liste des éléments à préparer avant la visite ;

- Organisation logistique et financière des activités.

\subsection{Messages clés}

Le coach : une aide, un appui (et non un évaluateur).

Le Formulaire : un outil pour améliorer la prise de décision et recadrer la gestion de l'aire protégée.

La mission de coaching : une opportunité pour réfléchir ensemble sur la gouvernance et la gestion de l'aire protégée ou du réseau d'aires protégées, et apporter des améliorations concrètes.
Ensemble : Le coach ne peut rien faire sans la collaboration étroite du Conservateur et de l'institution nationale.

II n’y a pas de résultat ni de radar correct. L'outil BIOPAMA permet de mieux analyser la situation actuelle et de proposer des améliorations pour le futur.

\subsection{Foire aux questions}

Les questions suivantes permettront au coach de répondre aux questions susceptibles d'être posées par ses interlocuteurs, aussi bien dans les premières étapes de contact que dans les interventions ultérieures.

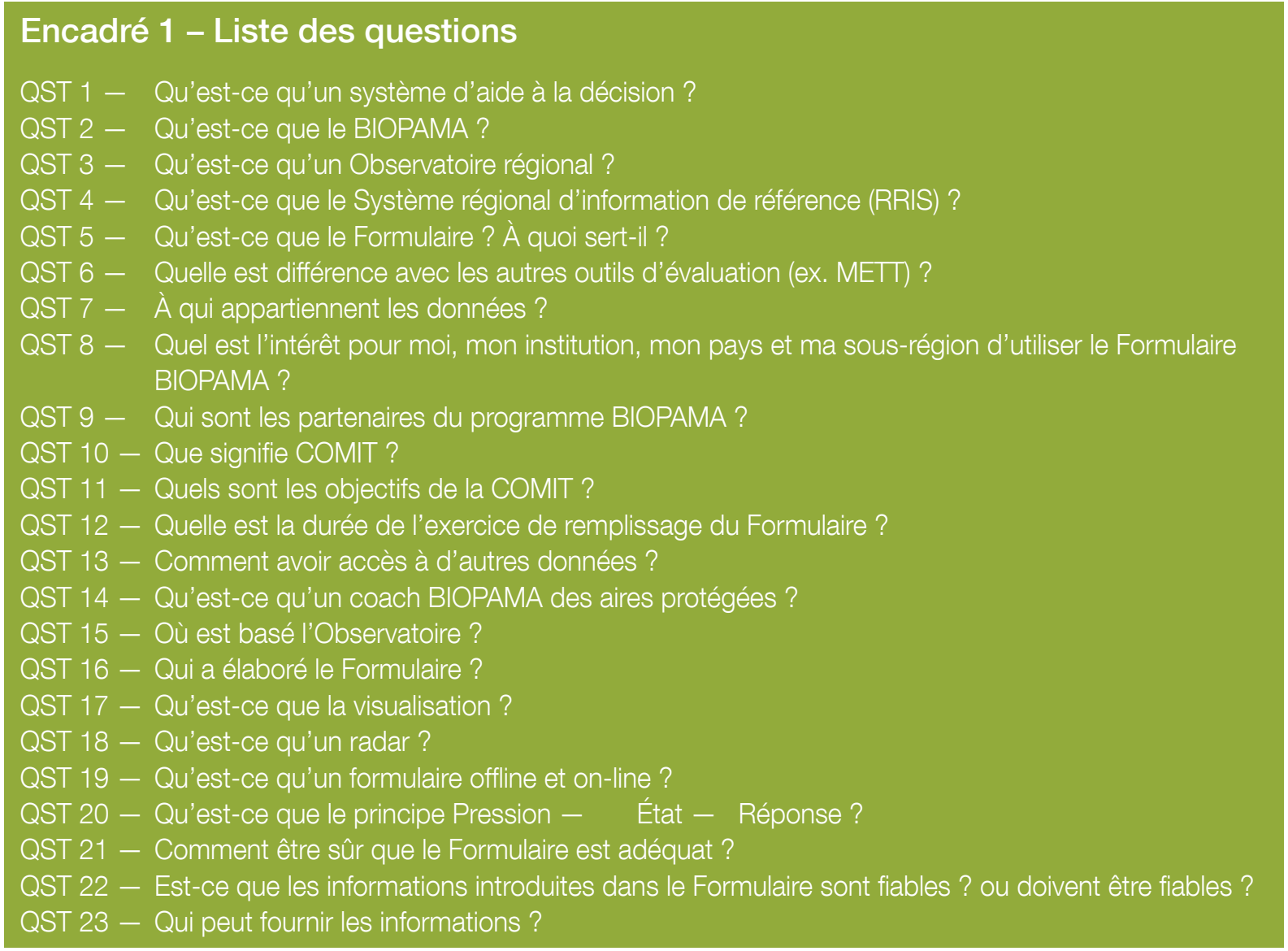




\section{QST 1 - Qu'est-ce qu'un système d'aide} à la décision?

Un système d'aide à la décision (Decision Support System - DSS) est destiné à faciliter la prise de décision. C'est un système qui couvre une large gamme de données et qui dispose de fonctions interactives pour structurer et organiser les données à la demande de l'utilisateur. Les meilleurs systèmes d'aide à la décision proposent des rapports synthétiques, des tableaux ou des graphiques qui facilitent l'interprétation d'informations pour la prise de décision.

II existe actuellement beaucoup d'informations disponibles sur les aires protégées, mais cela requiert du temps pour les chercher et des experts pour les analyser et les synthétiser pour permettre une application pratique. Or, les décideurs - aussi bien au niveau du site de l'aire protégée qu'au niveau national, régional ou global - ont besoin d'informations organisées et orientées vers la prise de décision. Le système d'aide à la décision leur permet de dégager, à partir de données brutes, l'essentiel des informations qui leur sont utiles et, par conséquent, d'adopter les solutions les plus appropriées et une approche proactive (par opposition à une intervention réactive). La planification, la gestion et le suivi de la biodiversité et des ressources naturelles imposent d'adopter des systèmes d'aide à la prise de décision.

Exemple de système d'aide à la décision : http://epi.yale.edu/

\section{QST 2 - Qu'est-ce que le BIOPAMA ?}

Le Programme pour la biodiversité et la gestion des aires protégées (BIOPAMA - Biodiversity and Protected Areas Management) cherche à lutter contre les menaces qui pèsent sur la biodiversité dans les pays d'Afrique, des Caraïbes et du Pacifique (ACP), tout en réduisant la pauvreté dans les communautés aux alentours et dans les aires protégées. Plus précisément, le Programme permettra d'améliorer l'accès aux connaissances scientifiques disponibles pour consolider le travail des institutions et des réseaux existants et renforcer les capacités, en vue d'améliorer les politiques et la prise de décision en matière de conservation de la biodiversité, de la gestion des aires protégées, et de l'accès et le partage des avantages.

Le BIOPAMA est une initiative de quatre ans (20122016) du Secrétariat ACP financé par I'Union Européenne.
Le programme BIOPAMA est une occasion à saisir pour :

- Établir des liens fonctionnels entre le personnel sur le terrain, les chercheurs et les décideurs et promouvoir des synergies parmi les professionnels de la conservation ;

- Résoudre les difficultés actuelles en établissant les priorités et orientant les actions au niveau local, national et régional ;

- Définir des objectifs mesurables pour les conditions de conservation souhaitées à partir de points zéro (lignes de base ou baselines) reliés ensemble par des valeurs de référence évolutifs ou graduels (benchmarks).

www.biopama.org

\section{QST 3 - Qu'est-ce qu'un Observatoire régional ?}

Les Observatoires régionaux pour les aires protégées et la biodiversité sont des centres de ressources régionaux pour améliorer la prise de décision pour la gouvernance et la gestion de la biodiversité et des ressources naturelles dans et en dehors des AP. Les Observatoires renforceront les capacités d'utilisation des données et les informations disponibles dans la conservation et la gestion des ressources naturelles. Les observatoires devront :

- Servir de centres de connaissances pour les régions ;

- Faciliter la mise en réseau des experts et des institutions ;

- Coordonner l'action politique et l'appui technique à l'intérieur des réseaux de conservation et entre les organisations nationales et régionales ;

- Garder les liens avec d'autres observatoires.

\section{QST 4 - Qu'est-ce que le Système} régional d'information de référence?

Le Système régional d'information de référence (RRIS, http://rris.biopama.org) du BIOPAMA est un système qui intègre une gamme diversifiée d'informations et de données pertinentes concernant les aires protégées et la biodiversité. II est construit grâce à un logiciel open-source et aux données de l'Observatoire numérique pour les aires protégées (DOPA), tout en disposant de son propre observatoire numérique. Les communautés de la biodiversité et de la conservation sont encouragées à l'utiliser et à participer au développement de ces outils.

http://dopa.jrc.ec.europa.eu 
QST 5 - Qu'est-ce que le Formulaire ? À quoi sert-il ?

Un Formulaire dénommé pour le moment " Formulaire BIOPAMA » a été établi pour permettre la collecte et l'organisation des données du contexte de l'intervention, et ainsi mesurer l'efficacité de la gestion des AP. Ce Formulaire BIOPAMA, ou plus simplement "le Formulaire " dans le présent document, s'inspire de plusieurs outils utilisés pour l'évaluation de l'efficacité de la gestion, par exemple : l'Outil de suivi de l'efficacité de la gestion (METT) ; la Mise en valeur de notre patrimoine $(\mathrm{EOH})$; la méthode d'Évaluation rapide et d'établissement des priorités de gestion des AP (RAPPAM) ; les indicateurs de synthèse et principaux identifiés par l'étude globale sur l'évaluation des aires protégées (Global study, Second edition - 2010) ; le Calculateur de menaces ; ou encore les propositions élaborées par le Centre conjoint de recherche avec l'Institut congolais pour la conservation de la nature (ICCN), l'Agence de coopération internationale allemande pour le développement (GIZ), le Réseau des aires protégées d'Afrique centrale (RAPAC) et l'Observatoire des forêts d'Afrique centrale (OFAC). II se base également sur des analyses de spécialistes dans le domaine, et des propositions élaborées sur le sujet par différents apports et contributions de la part de gestionnaires d'AP.

Le Formulaire BIOPAMA vise à :

- Établir les conditions d'intervention dans la gestion des AP avec des informations organisées sur une base commune et selon la logique Contexte - Gestion - Gouvernance ;

- Combler ou mettre en évidence les lacunes en matière d'informations prioritaires et indispensables à la gestion et à la gouvernance des AP ;

- Orienter les interventions selon la logique Pression - État - Réponse (voir QST 20) permettant d'améliorer la vision, la planification, la gestion, l'évaluation et le suivi dans la réalisation des actions en faveur de la conservation et de la gestion/valorisation des ressources naturelles ;

- Adopter une approche orientée sur les résultats et sur l'impact qui est supportée par la détermination des indicateurs et des valeurs de référence (benchmarks).

Le Formulaire n'est pas un outil scientifique pour la recherche, mais un outil de décision!
QST 6 - Quelle est différence avec les autres outils d'évaluation?

Le Formulaire BIOPAMA s'inspire de plusieurs outils d'évaluation de l'efficacité de la gestion des aires protégées largement utilisés dans le monde tels que ceux de l'Évaluation rapide et l'établissement des priorités de gestion des AP (RAPPAM), I'Outil de suivi de l'efficacité de la gestion (METT) ou la Mise en valeur de notre patrimoine $(\mathrm{EOH})$. II est basé sur les recommandations de la Commission mondiale des aires protégées (CMAP) sur le cadre d'évaluation de l'efficacité de la gestion des aires protégées. Ses résultats peuvent être convertibles en valeur dans l'Outil de suivi de l'efficacité de la gestion des aires protégées (PAMETT) ou l'Outil de suivi de l'efficacité de la gestion (METT) grâce au convertisseur vers le METT, et grâce aussi au tableau des indicateurs de synthèse et principaux de l'Étude mondiale (Leverington et al., 2010). Grâce à l'approche de cycle de gestion d'une AP, il permet de détecter les problèmes de gestion sous différents angles et amènent plus aisément à la formulation de propositions d'améliorations.

\section{QST 7 - A qui appartiennent les données?}

Toutes les données et informations transmises à l'Observatoire sont soumises à la licence Creative Commons (http://creativecommons.org/licenses/ by-nc-sa/4.0/deed.fr). Selon cette licence, les données appartiennent à celui qui les a produites. En les encodant dans le Formulaire, l'offrant accorde à l'Observatoire le droit de reproduire, distribuer et communiquer ces données au public à des fins non commerciales. L'Observatoire s'engage toutefois à citer le nom de l'auteur original de la manière indiquée par l'auteur de l'œuvre ou le fournisseur des données. Si l'Observatoire modifie, transforme ou adapte cette création, il n'a le droit de distribuer la création qui en résulte que sous un contrat identique à celui-ci. Les utilisateurs des données et informations sont soumis à cette même licence Creative Commons.

QST 8 - Quel est l'intérêt pour moi, mon institution, mon pays et ma sous-région d'utiliser le Formulaire BIOPAMA ?

Le Formulaire permet de :

- Représenter une synthèse très opérationnelle de la planification de la gestion ;

- Constituer une base de référence pour la gestion, en l'absence d'instruments de planification à moyen et long terme ; 
- Assurer la cohérence et la continuité par rapport aux instruments de planification annuelle ;

- Disposer d'un outil objectif de suivi et d'autoévaluation des processus de gestion et de gouvernance des aires protégées ;

- Établir une notification normalisée ;

- Afficher les résultats des efforts de conservation selon des normes reconnues.

Lors des phases test du Formulaire, l'exercice de remplissage du formulaire par les gestionnaires d'AP s'est révélé être une opportunité pour discuter des opinions de chacun, déceler des faiblesses et des possibilités d'amélioration de la gestion et de la gouvernance de l'AP.

\section{QST 9 - Qui sont les partenaires du programme BIOPAMA?}

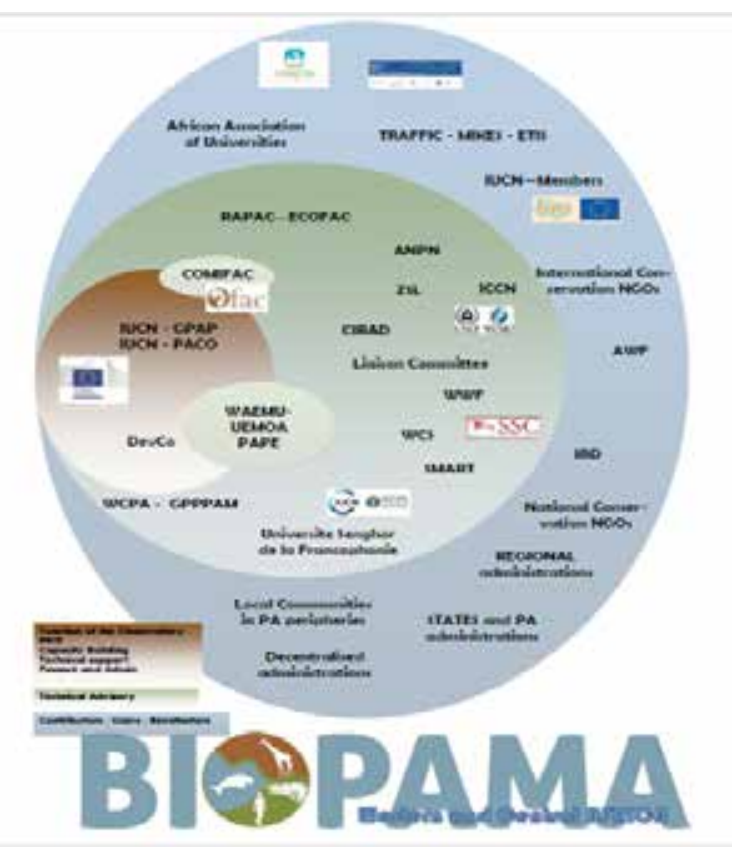

\section{QST 10 - Que signifie COMIT ?}

Mallette pédagogique pour effectuer la mission de coaching et développer le système d'information de l'Observatoire des aires protégées et de la biodiversité du programme BIOPAMA (en anglais, coach Observatory Mission Information Toolkit)

\section{QST 11 - Quels sont les objectifs de la COMIT ?}

Objectif général : Support didactique visant à aider les coaches à accompagner les acteurs de la conservation dans l'utilisation du système d'aide à la décision proposé par l'Observatoire régional des AP dans la gestion et la gouvernance des AP en Afrique centrale et occidentale.

\section{Objectifs spécifiques :}

- Fournir aux coaches les ressources nécessaires pour former et accompagner les gestionnaires des aires protégées mobilisés dans la campagne de renforcement des capacités du BIOPAMA ;

- Planifier les étapes pratiques pour la formation et l'accompagnement des gestionnaires ;

- Assurer une même norme de qualité dans les missions de coaching pour l'Observatoire régional des aires protégées et de la biodiversité.

\section{QST 12 - Quelle est la durée de l'exercice de remplissage du Formulaire?}

La durée du remplissage du Formulaire varie beaucoup en fonction de l'aire protégée, de la disponibilité et de l'accessibilité de l'information, et du degré de maîtrise de la gestion par les techniciens de I'AP. Généralement, le premier remplissage du Formulaire BIOPAMA demande plus de temps car il faut familiariser les acteurs à l'outil et au remplissage. Les révisions périodiques de l'état de gestion et gouvernance exigeront environ la moitié du temps passé la première fois.

Lors des phases tests du Formulaire, le premier remplissage a demandé entre 16 et 32 heures de travail (soit entre 2 et 4 jours), avec la présence d'un expert du Formulaire.

Certaines considérations techniques peuvent accélérer le remplissage, par exemple un pré-remplissage déjà effectué à l'avance, ou de bonnes conditions de travail (utilisation d'un vidéoprojecteur, mise à disponibilité de personnes-ressources).

\section{QST 13 - Comment avoir accès à d'autres données?}

En tant que centre de ressources, l'Observatoire régional permet d'accéder à d'autres informations et données issues d'autres aires protégées. II donne également des références sur d'autres sites ou bases de données. Les données seront visibles par n'importe qui sur le site internet de l'Observatoire et la grande majorité seront téléchargeables en format .pdf ou Excel. Le BIOPAMA RRIS intègre le logiciel GeoNode à partir duquel les données peuvent être déchargées. Ce logiciel (GeoNode, http://geonode-rris.biopama. org/) est actuellement géré de manière centralisée pour toutes les régions d'Afrique, des Carailbes et du Pacifique, mais il est prévu de développer des accès régionaux. 
QST 14 - Qu'est-ce qu'un coach BIOPAMA des aires protégées?

Le coach des aires protégées, établi dans le cadre du Programme BIOPAMA, est une personneressource de l'administration, ou du ressort des partenaires de la conservation, ou encore un expert indépendant ayant suivi une formation de coaches pour soutenir la diffusion et l'utilisation du système d'aide à la décision relayé par l'Observatoire régional des aires protégées et de la biodiversité.

\section{QST 15 - Où est basé l'Observatoire ?}

L'Observatoire est un système d'information et d'aide à la décision, accessible en ligne. Dans chaque sous-région, l'Observatoire est hébergé par une institution hôte. En Afrique centrale, il se trouve auprès de l'Observatoire des forêts d'Afrique centrale (OFAC) à Yaoundé, sous les auspices de la COMIFAC. En Afrique de l'Ouest, il est rattaché à l'Union économique et monétaire ouest-africaine (UEMOA).

\section{QST 16 - Qui a élaboré le Formulaire?}

Le Formulaire a été élaboré par le Centre commun de recherche (CCR) de la Commission européenne, avec la collaboration du RAPAC et OFAC, sur la base d'un travail de réflexion conduit conjointement sur le terrain et auprès du CCR, partant des différents outils existants pour le suivi et l'évaluation de la gestion des aires protégées (cfr. QST 5). La conceptualisation et le montage de l'outil « Formulaire BIOPAMA » ont été réalisés par C. Paolini et P. Roggeri, avec la collaboration technique de P. Mayaux, F. Palla et C. de Wasseige. Le développement informatique a été assuré par A. Marelli, avec l'appui de B. Djomo et D. Djossi. La partie statistique a été supervisée par P. Bialowolski. Des experts indépendants et des professionnels d'institutions en charge des aires protégées de la sous-région Afrique centrale ont contribué aux premières réflexions sur la mise en place d'une liste d'indicateurs utiles au suivi de la situation (état et gestion) des aires protégées, à l'occasion des ateliers organisés par le RAPAC et l'OFAC en 2008 et 2009. Ils ont aussi contribué à l'amélioration des premières versions de l'outil "Formulaire BIOPAMA » conçu par le CCR, à l'occasion des phases tests réalisées en 2014 et 2015 en RDC et au Gabon.

\section{QST 17 - Qu'est-ce que la visualisation?}

Le Formulaire permet de visualiser l'évaluation des éléments du cycle de gestion sous forme de radar, qu'il considère comme une aide à la décision.

La visualisation est une représentation, à l'aide de graphiques, de l'état du contexte de l'intervention et de l'évaluation des éléments du cycle de gestion. La visualisation permet d'afficher et de résumer les résultats des analyses, cependant elle n'assure qu'une représentation et doit être utilisée comme support et facilitation pour les réflexions en aide à la prise de décision.

\section{QST 18 - Qu'est-ce qu'un radar?}

Le radar est un graphique qui permet de visualiser l'évaluation des éléments du cycle de gestion comme aide à la décision (voir QST 17). II se construit au fur et à mesure que le Formulaire est rempli.

Pourquoi avoir choisi le radar pour la visualisation ? Le radar est la forme qui représente le mieux le système de gestion : 1) aspect circulaire 2) il montre les 6 aspects du cycle de gestion. Enfin, son côté basique facilite la lecture.

\section{QST 19 - Qu'est-ce qu'un formulaire offline et online?}

Le Formulaire BIOPAMA dans son format numérique est un petit logiciel qui peut être rempli offline et online. Le Formulaire offline est disponible sur clé USB et se remplit sans connexion Internet. Cela permet à tout gestionnaire, même sans accès à Internet, d'évaluer l'efficacité de la gestion de son site de conservation. Les résultats du Formulaire offline peuvent ensuite être synchronisés avec la base de données en ligne. Cependant, nous conseillons à tous les gestionnaires qui disposent d'une connexion internet d'utiliser le formulaire en ligne en se connectant sur http://www. observatoire-comifac.net/admin/home.php. II faut bien sûr avoir les droits d'encodage pour une ou plusieurs aire(s) protégée(s) pour que le formulaire puisse être utilisable.

\section{QST 20 - Qu'est-ce que le principe Pression- État - Réponse?}

Le formulaire permet d'analyser les problèmes environnementaux selon le principe Pression - État Réponse (Pressure - State - Response). 
Selon ce principe, les activités humaines exercent des pressions sur l'environnement (par ex. la pollution ou le changement d'utilisation des sols), qui peuvent induire des changements sur l'état de l'environnement (par ex. sur les niveaux de polluants ambiants, ou la diversité des habitats). La société ou une organisation répond à ces changements à travers des stratégies et des programmes qui visent à prévenir, à réduire ou à atténuer ces pressions et/ou les dommages environnementaux. Ce principe a évolué et inclut désormais les facteurs/forces motrices et les impacts : Facteurs - Pression-État-Impact-Réponse (FPEIR).

La fiche 5 approfondit ce principe dans le cadre de la gestion des aires protégées.

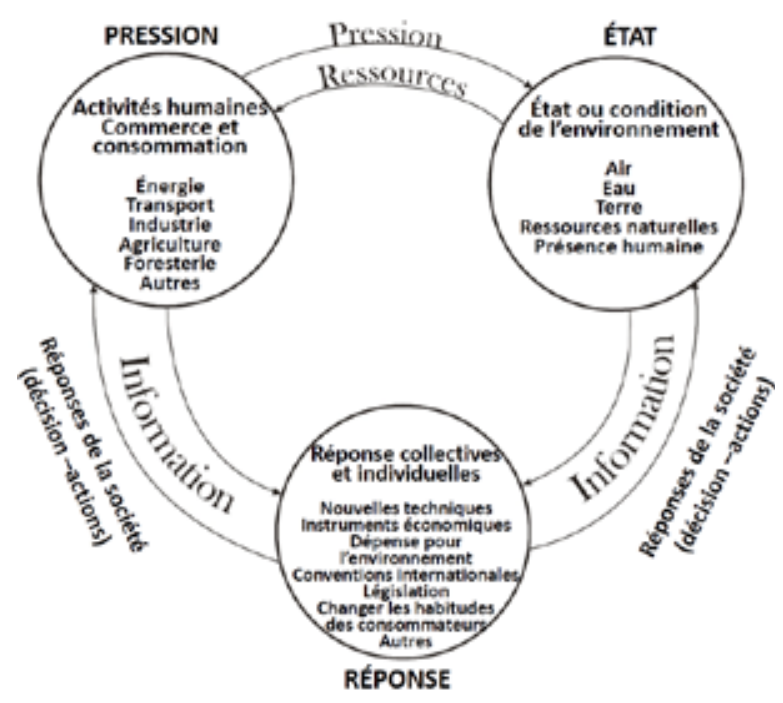

Source : http://www.fao.org/ag/againfo/programmes/ en/lead/toolbox/Refer/Envlndi.htm\#DPSIR

\section{QST 21 - Comment être sûr que le Formulaire est adéquat?}

Le Formulaire BIOPAMA s'inscrit dans le Cadre d'évaluation de l'efficacité de la gestion élaborée par la Commission mondiale des aires protégées (Hockings et al, 2008). Ce cadre commun ou méthodologie commune a été pensé par plusieurs experts et a fait ses preuves. Le Formulaire s'inspire de ces différentes méthodologies d'évaluation largement utilisées dans le monde.

Voir aussi QST 5, QST 6, QST 8.
QST 22- Est-ce que les informations introduites dans le Formulaire doivent être fiables?

Le Formulaire n'est pas un outil scientifique qui donnerait les résultats de la recherche. Certaines informations peuvent être extraites de rapports de recherche ou de publications. Toutefois, et souvent dans l'ensemble des cas, les informations sur la gestion dépendront delaperceptiondu Conservateur, de l'équipe de gestion et des partenaires présents lors du remplissage. C'est un outil qui va aider toutes les parties prenantes à la gestion à définir certaines orientations. Ainsi l'absence d'informations ou de données scientifiques ne doit pas être un obstacle au remplissage.

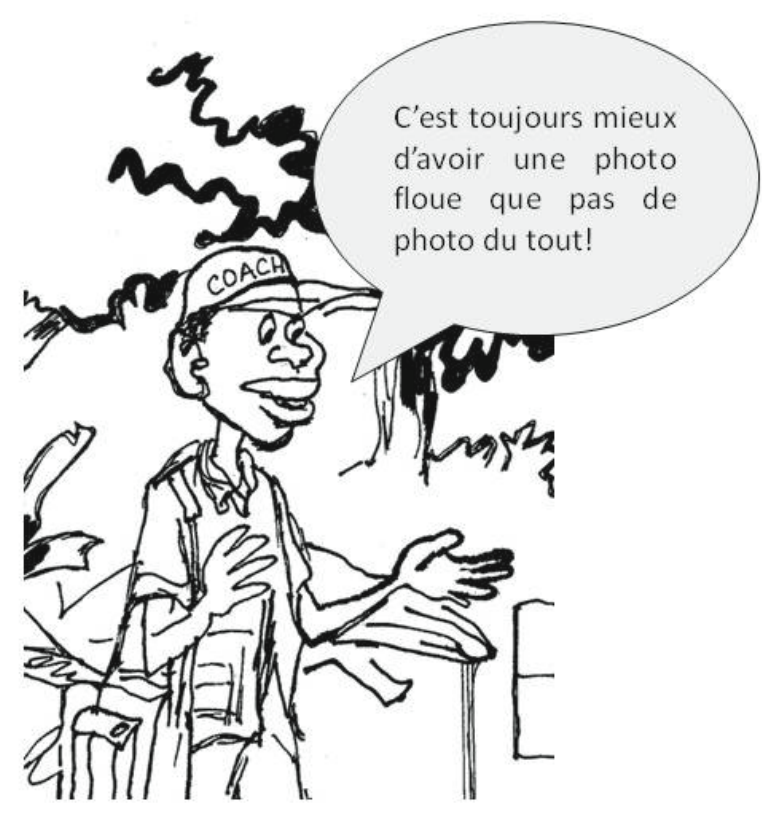

\section{QST 23 - Qui peut fournir les informations?}

Les informations à fournir lors du remplissage $\mathrm{du}$ Formulaire proviennent principalement $\mathrm{du}$ Conservateur et de son équipe de gestion (notamment le responsable du suivi-évaluation, la direction technique et des opérations au niveau de l'institution nationale), et éventuellement de certains partenaires locaux. Si toutefois le Conservateur et son personnel sont nouveaux, deux personnes ressources indiquées par l'administration nationale pourront les aider. 
Liens web et bibliographie

Site du Programme BIOPAMA

http://biopama.org/

Site de l'OFAC

http://www.observatoire-comifac.net/

Observatoire numérique de la Commission

Européenne

http://dopa.jrc.ec.europa.eu

Base de données mondiale sur les aires protégées http://www. protectedplanet.net/

\section{Fichiers dans la clé USB}

0-1Biopama_fact_sheet_fr_version

0-2Biopama_presentation_ppt_fr_

0-3 Système d'information DSS

\section{En cas de difficultés}

Pour les questions relatives à :

L'UICN et les partenariats du Programme BIOPAMA

Sébastien Régnaut, coordinateur régional de l'UICN Afrique centrale et occidentale,

Ouagadougou - Burkina Faso

Sebastien.Regnaut@iucn.org

Traitement et analyse des données :

Donald Jomha Djossi, COMIFAC-OFAC

ddjossi@observatoire-comifac.net

Pour la mise en œuvre du Programme BIOPAMA paco@iucn.org

Domoina Rakotobe

domoina.rakotobe@ext.iucn.org 


\section{PARTIE 2}

\section{Comment organiser le coaching?}

\section{QUAND UTILISER CETTE PARTIE :}

- Lors des premiers entretiens et réunions sur le Système d'information ou le Programme BIOPAMA

- Avant les ateliers de formation et les missions sur le terrain

COMMENT : Avec le binôme. En étroite collaboration avec l'institution de tutelle de l'aire protégée.

DURÉE : Dépend du degré de familiarité avec l'institution. Prévoir deux mois avant le début des activités.

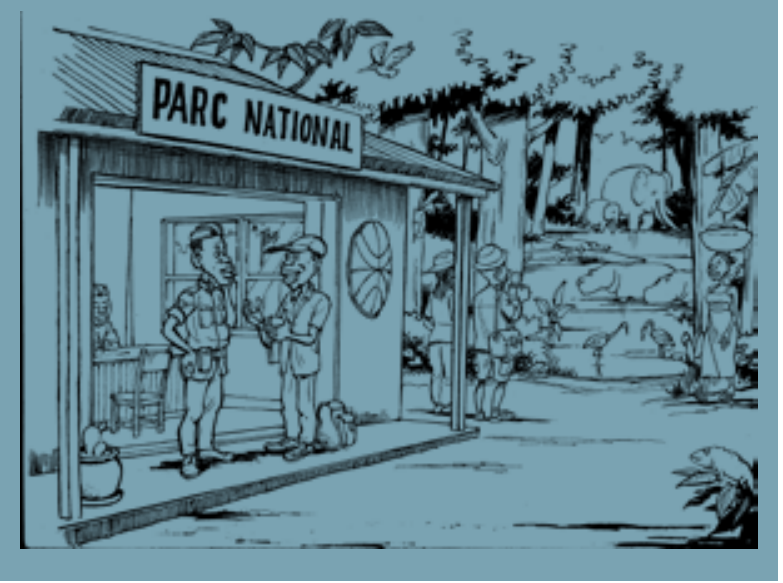

\section{1 Être coach BIOPAMA des aires protégées}

\subsubsection{Définition de la mission de coaching}

(1) L'instauration des coaches des aires protégées fait suite à un changement de paradigme dans le développement des capacités des gestionnaires des aires protégées. Le coach remplace le formateur - lequel est souvent lié à la transmission de connaissances et de techniques dans une période déterminée. En revanche, le coaching reflète un accompagnement dans le temps et de nouvelles relations d'entraide. La formation telle qu'on l'entend traditionnellement n'est pas exclue dans le processus de coaching mais elle est davantage centrée sur les besoins des gestionnaires des aires protégées. Le coach va aider à développer les nécessités de production de données, d'organisation des informations, d'analyse des états et des efforts de conservation et de formulation de propositions d'interventions. Le coaching vise aussi à renforcer les capacités intangibles qui auront des répercussions sur le long terme telles que la capacité d'analyse, de plaidoyer, d'utilisation des ressources existantes,

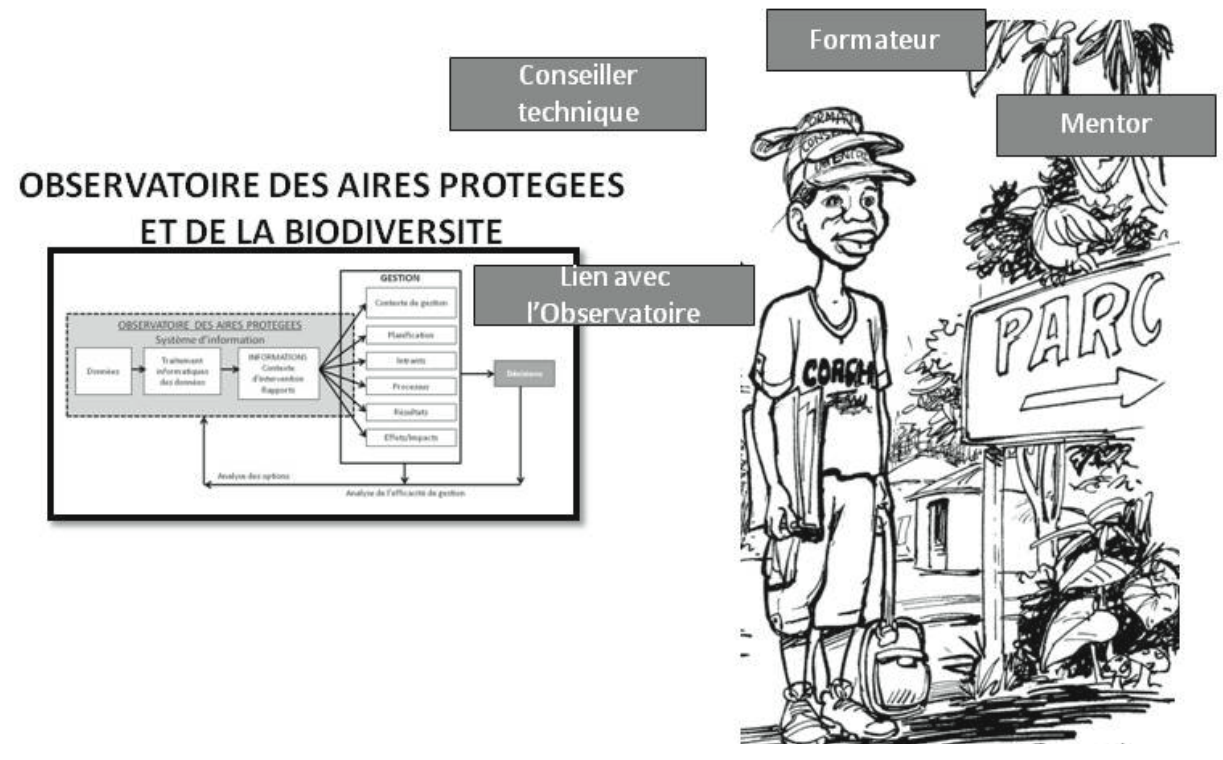


Le coaching est l'accompagnement des gestionnaires des aires protégées pour l'amélioration de l'efficacité de la gestion des AP d'Afrique centrale et occidentale par le développement de leurs potentiels et de leurs savoir-faire dans le cadre de l'utilisation du Système d'aide à la décision.

etc. Dans un premier temps, environ 25 coaches, issus d'une dizaine de pays d'Afrique centrale et occidentale, seront formés à l'utilisation de la Mallette pédagogique lors d'un atelier régional.

La charte du coach formule des points de repère déontologiques, compte tenu des spécificités du coaching en tant que processus d'accompagnement professionnel.

\subsubsection{Les attentes}

Il est attendu du coach de :

- Participer au niveau de chaque pays à I'identification du programme d'amélioration de la gestion et de la gouvernance des AP, en définissant les priorités d'intervention ;

- Introduire et former les équipes de gestion des
AP sélectionnées à l'utilisation du Formulaire de l'Observatoire, et analyser les résultats pour l'amélioration de la gestion et la gouvernance des AP et le développement du système de planification-suivi-évaluation interne. Ceci peut se faire sous forme d'atelier de formation ;

- Assister au niveau de chaque AP les équipes de gestion à l'utilisation du Formulaire et à l'analyse des résultats ;

- Suite à l'utilisation du Formulaire, soutenir les équipes de gestion des AP et l'institution de tutelle à l'élaboration de propositions d'amélioration de gestion, de suivi et de planification (plan de gestion et d'action - plan de travail) ;

- Assurer les restitutions basées sur les analyses de l'efficacité de gestion à la fin de chaque étape d'intervention ;

- Participer au réseau d'échange sur l'utilisation et l'amélioration de l'outil « Formulaire BIOPAMA »;

Jusqu'où va la mission de coaching ? II faut faire attention à ne pas s'engager dans des activités qui dépassent le cadre de la mission tel que le renouvellement du plan de gestion en entier.

\section{Encadré 2 - La charte du coach BIOPAMA des aires protégées}

\section{CHARTE DU COACH DES AIRES PROTEGÉES}

Titre 1 - Devoirs du coach

Art. 1-1 - Exercice du coaching : Le coach de l'Observatoire a été mandaté par son institution pour exercer cette fonction à partir de sa formation, de son expérience et de sa supervision.

Art. 1-2 - Confidentialité : Le coach s'astreint au secret professionnel. II consent à ne pas divulguer à des tiers des informations confidentielles auxquelles il pourrait avoir accès au cours de sa mission.

Art. 1-3 - Respect des personnes : Conscient de sa position, le coach s'interdit d'exercer tout abus d'influence et adopte le principe de la reconnaissance et du respect des autres.

Titre 2 - Devoirs du coach vis-à-vis de l'équipe de gestion (le « coaché »)

Art. 2-1 - Responsabilité des décisions : Le coaching est une technique de développement professionnel et personnel. Le coach abandonne de ce fait toute la responsabilité des décisions au coaché : à savoir le Conservateur, son équipe, ses partenaires de gestion ainsi que l'institution de rattachement.

Art. 2-2 - Protection des personnes et de l'organisation : Le coach adapte son intervention dans le respect des étapes de développement du coaché. Le coach est attentif au métier, aux usages, à la culture, au contexte et aux contraintes de l'organisation dans laquelle il est assigné. En particulier, le coach garde une position extérieure à l'organisation, ne prend pas position, et ne s'ingère pas dans des questions internes.

Art. 2-3 - Équilibre de l'ensemble du système : Le coaching s'exerce dans la synthèse des intérêts du coaché et de son organisation.

Adaptée de la charte de l'Association européenne de coaching et de la Société française de coaching 
- Contribuer à l'établissement des liens fonctionnels entre les réseaux des AP et l'Observatoire ;

- Organiser la restitution des résultats des missions de coaching auprès de l'institution de tutelle, des acteurs de l'aire protégée et du Programme BIOPAMA ;

- Contribuer àl'intégration du système d'information d'aide à la décision dans leur institution nationale.

Spécifiquement en tant que coach, il/elle aura la charge de :

- Encourager l'équipe de gestion dans le processus de familiarisation au Système d'information ;

- Répondre aux questions du coaché et fournir des orientations relatives à l'utilisation du
Système d'information et au développement des compétences ;

- Développer l'esprit d'équipe lors du remplissage du Formulaire BIOPAMA et de l'analyse des résultats de gestion et de gouvernance ;

- Gérer les interventions des différentes parties prenantes dans l'utilisation du Formulaire BIOPAMA ;

- $\quad$ Stimuler et favoriser l'esprit analytique à partir de preuves factuelles avec les équipes de gestion de l'AP ;

- Développer l'habilité du coaché à prendre des décisions et à réagir sur des problèmes spécifiques de gestion de l'aire protégée.

Tableau 1 - Chronogramme général de la mission de coaching

\begin{tabular}{|c|c|c|c|c|c|c|}
$\begin{array}{c}\text { Introduction de } \\
\text { la mission }\end{array}$ & $\begin{array}{c}\text { Formations des } \\
\text { gestionnaires }\end{array}$ & \multicolumn{3}{|c|}{$\begin{array}{c}\text { Missions sur sites Accompagnement Restitutions } \\
\text { pour chaque AP }\end{array}$} & $\begin{array}{c}\text { Restitutions } \\
\text { nationales } \\
\text { Rapports divers }\end{array}$ \\
\hline Mois & $\mathbf{1}$ & 2 & 3 & 4 & 5 & 6 \\
\hline
\end{tabular}

\section{Encadré 3 - Les capacités importantes}

Les capacités moins évidentes mais importantes pour développer le potentiel humain de l'Afrique

- Capacité à fournir un leadership visionnaire et stratégique ;

- Capacité à apprendre, à se concentrer et à élaborer des stratégies ;

- Capacité à exploiter et à utiliser efficacement les capacités et forces existantes ;

- Capacité à retenir, embaucher et utiliser efficacement un personnel compétent et productif ;

- Capacité à utiliser les potentiels sous-utilisés au sein d'une organisation ;

- Capacité à prévoir, adapter et répondre à un environnement volatile et en perpétuelle évolution ;

- Capacité à exploiter la créativité et l'innovation ;

- Capacité à fournir un espace pour le personnel par les gestionnaires et la capacité d'utiliser cet espace par le personnel (émancipation) ;

- Capacité à motiver et inspirer le personnel :

- Capacité à inculquer un plus grand sentiment d'appartenance parmi le personnel pour atteindre les objectifs organisationnels fixés ;

- Capacité à assurer la redevabilité et la responsabilité mutuelle ;

- Capacité à communiquer efficacement avec des audiences internes et externes ;

- Capacité à apprendre et à appliquer les leçons apprises pour améliorer les performances pour la prestation efficace de services, et pour ajuster et prendre des mesures correctives ;

- Capacité à suivre et à évaluer l'impact.

Source : NEPAD, 2009. The AU/NEPAD Capacity Development Strategic Framework. Seeing African People as the true Resource. Johannesburg : NEPAD.

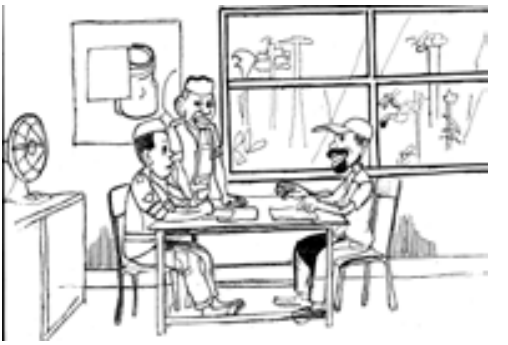

\section{QUESTIONS POUR LE COACH}

Comment comprenez-vous votre mission de coach ?

Avez-vous reçu l'aval de votre institution pour effectuer le rôle de coach pour l'Observatoire des aires protégées? 


\subsubsection{Binôme de coach}

(耳) Chaque pays considéré dans la campagne BIOPAMA de renforcement des capacités pour une meilleure gestion des aires protégées dispose de deux coaches qui vont former un binôme. Toutes les interventions devront être organisées et mises en œuvre ensemble par les deux coaches, dans la complémentarité de leurs expériences.

Le binôme est établi pour : i) assurer la continuité de l'exercice ; ii) se partager les tâches importantes au cours de la phase de terrain; et enfin iii) assurer la qualité dans le remplissage du Formulaire BIOPAMA.

Pour garantir la qualité au cours du remplissage du Formulaire, il est impératif de permuter les rôles entre

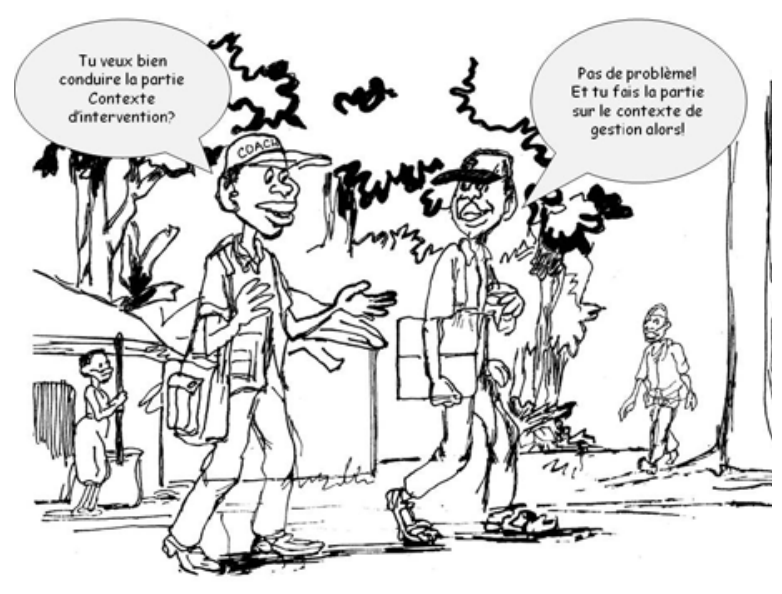

les deux coaches : l'un joue le rôle de coach leader et l'autre celui de réviseur. Le binôme a la liberté de s'organiser comme il l'entend.
Coach leader (ou principal)

- Dirige la session d'utilisation du Formulaire

- Interagit avec les acteurs de la conservation ;

- Suit le remplissage du Formulaire ;

- Encourage les échanges et les analyses.

\section{Coach réviseur (ou assistant)}

- Vérifie la qualité et la logique de l'exercice ;

- Aide à recentrer les discussions ;

- Fournit des informations ou des explications supplémentaires ;

- Soutient les participants en difficulté ;

- $\quad$ Prend des notes si nécessaire.

\subsection{Techniques de coaching et d'animation}

\subsubsection{Principes de coaching}

Le coaching part du principe que les personnes sont compétentes pour trouver les solutions à leurs problématiques. II permet d'accéder et de réactiver les ressources dont elles disposent déjà. Le coaching vise l'autonomie de la personne afin qu'elle puisse continuer une sorte d'auto-coaching après les séances de coaching.

Le coaching ouvre de nombreuses opportunités, il permet entre autres de :

- Recevoir le soutien et l'encouragement d'un pair ou d'un expert tout en évaluant les expériences, discutant des sentiments, décrivant les frustrations, et vérifiant les perceptions ;

- Affiner des stratégies ou des techniques à travers les remontées d'informations, et une assistance techniques ;

- Analyser les pratiques et amener la prise de décision à un niveau conscient ;

- Adapter ou généraliser les compétences ou les stratégies en tenant compte de ce qui est nécessaire pour améliorer les résultats ;
- Réfléchir sur les perceptions et/ou la façon dont les décisions sont prises, ce qui améliore la connaissance et la compréhension des pratiques professionnelles.

II n'existe pas un modèle unique de coaching. Chaque cas implique un objectif distinct, un rôle particulier pour le coach ainsi qu'un type de relations entre le coach et le coaché. Les coaches techniques aident à la maitrise d'une nouvelle technique ou procédé. Certains coaches aident à trouver une solution à un problème spécifique. D'autres vont mettre en place des pratiques auto-réflexives destinées à aider le coaché à réfléchir sur ses propres pratiques pour améliorer sa performance professionnelle. Enfin, d'autres coaches peuvent consolider l'esprit d'équipe d'une entreprise. Ces formes de coaching se retrouvent parfois ensemble, à des degrés divers. Elles impliquent l'utilisation d'une gamme de techniques, qui varient en fonction de la culture et des moyens disponibles.

Le coaching est l'art de poser les questions et de remettre en cause les hypothèses, et non de dire à l'autre ce qu'il doit faire. 
Encadré 4 - Les modèles de coaching les plus efficaces

Sur site : le coaché veut voir le coach faire la pratique lui-même, dans son milieu de travail, et voir qu'une technique est non seulement faisable mais bien faite.

Bien équilibré : en termes d’interventions. Le coaché ne doit se sentir ni opprimé ni délaissé.

Les bons coaches ne dictent pas : ils facilitent la réflexion et la prise de responsabilité des autres, ils sentent quand encourager le coaché et quand rester en arrière.

Des remontées d'informations efficaces, c'est-à-dire :

- Descriptives ( $\neq$ évaluatives) ;

- Spécifiques ( $\neq$ générales) ;

- Décrivent des évènements ou des comportements observables, au lieu de donner des opinions personnelles ;

- Se focalisent sur les comportements ( $\neq$ la personne) ;

- Partagent des informations au lieu de donner des avis ;

- Explorent d'autres alternatives au lieu de donner une solution (ou la solution) ;

- Commencent avec un point positif ;

- Décrivent des relations observables entre les comportements ou les évènements pour que le coaché puisse lui-même faire les relations de cause à effet ;

- Offre des informations que l'interlocuteur peut utiliser.

Favoriser les réflexions professionnelles en encourageant les analyses, l'auto-évaluation, les discussions avec les pairs ou une communauté de praticiens sur des problématiques et non sur un fait particulier.

Les coaches doivent être bien formés : à la fois sur les techniques de communication interpersonnelle, la maîtrise du domaine d'action (ici la gestion d'une aire protégée), les techniques de coaching.

Collaboratif : en permettant la collaboration avec le coaché, entre coaches, et avec les autres partenaires. Le coach n'a pas l'obligation de tout faire et encore moins de tout connaître.

Source: Koh, S. \& Neuman, S.B. (2006). Exemplary elements of coaching : Ann Arbor MI: University of Michigan Research Program on Ready to Read.

\subsubsection{Techniques de base du coaching}

Toute activité de coaching doit inclure les éléments suivants :

- Un accompagnement ;

- Une remontée d'informations (feedback) ;

- L'analyse de l'application d'une technique ou d'une stratégie ;

- La facilitation personnelle ;

- L'adaptation au rythme, à la culture et au style d'apprentissage du coaché.

Ces éléments impliquent quatre activités de base : l'observation, la démonstration, la communication et la remontée d'informations (feedback). II n'y a pas de directive précise sur l'ordre ou la fréquence de ces quatre activités.

\section{Techniques d'observation}

Le coach doit observer le coaché pour comprendre ses forces et ses faiblesses.

- Observation de la personne : son attitude, ses réactions, son style d'apprentissage, ses relations avec les autres ;

- Observation technique : comprenant à la fois la démarche méthodologique (ou processus) et les résultats.

\section{Techniques de démonstration}

II s'agit de faire, et non de "faire faire ". Le coach doit être capable d'utiliser les outils et d'expliquer les différentes étapes de manipulation d'un outil ou d'un procédé. En effet, s'il reste uniquement dans une position d'observateur ou de juge, une distance va se créer avec le coaché. 


\section{Techniques de communication et de remontée d'informations /feedback}

La communication est fondamentale dans la pratique du coaching. Selon le modèle de coaching utilisé, le coach doit assumer plusieurs fonctions de communication en :

- Fournissant des informations théoriques et pratiques ;

- Faisant remonter les informations et en donnant des suggestions ;

- Incitant à la réflexion sur la pratique.

\section{(1) Comment faire face à la réticence des gestionnaires à remplir le Formulaire ?}

Même si la mission de coaching a été validée par la direction nationale, il est possible que le Conservateur soit réticent à remplir correctement le Formulaire pour différentes raisons (le temps que cela prend, l'urgence d'autres affaires plus importantes...). Une astuce pour maintenir son attention et favoriser son engagement : prenez l'exemple d'une demande de financement dans une banque, il faut faire un dossier - cela prend du temps, c'est complexe, mais on peut anticiper les résultats.

\section{Comment réduire la peur d'être sanctionné ou noté ? Comment diminuer l'attitude défensive du Conservateur?}

Il est important de clarifier dès le départ et de rappeler fréquemment que l'exercice a pour but d'améliorer la gestion de l'aire protégée. Ayez une attitude collaborative. En effet, devant un coach enclin aux critiques et au jugement, le Conservateur pourrait avoir l'impression d'être jugé par quelqu'un d'externe - devant son personnel et ses partenaires qui plus est. C'est l'efficacité de la gestion de l'aire protégée qui sera évaluée et non le personnel. II revient donc au coach d'instaurer un climat de confiance, détendu et collaboratif avant le début de l'atelier de remplissage.

Sources : American Institute of Research (2005) Conceptual Overview : Coaching in the Professional Development impact Study.

Koh, S. \& Neuman, S.B. (2006). Exemplary elements of coaching : Ann Arbor Ml: University of Michigan Research Program on Ready to Read.

\section{Exercice 1 - Philosophie du coaching}

Avant de coacher les autres, le coach doit se connaître et savoir définir ce qui le motive à faire du coaching. Cet exercice, à remplir par le coach - et à relire tout au long de la mission de coaching - peut aider à mieux définir les valeurs et les objectifs du coaching en termes personnels. La philosophie de coaching peut évaluer au fil du temps.

Les valeurs qui me poussent à faire du coaching :

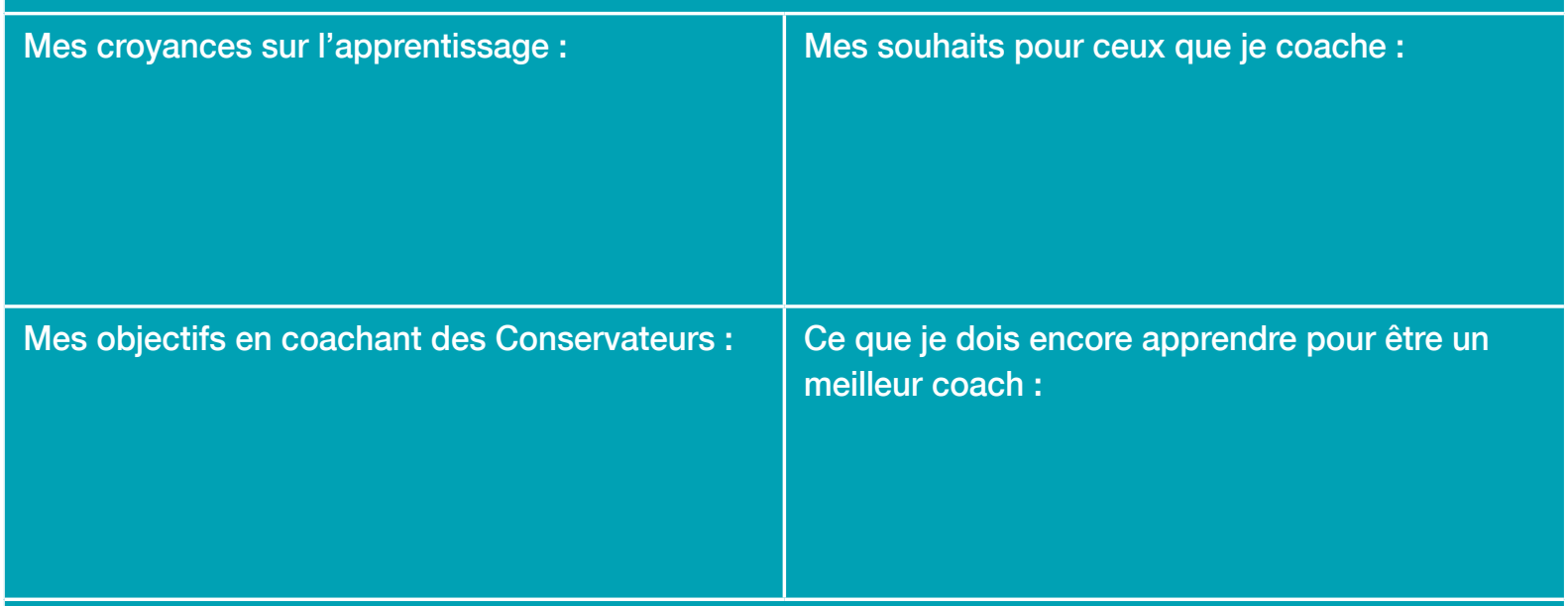

Source : Psencik K (2015) Philosophy of Coaching JSD Fev, 36 (1), p56-57 


\section{PROTOCOLE DES MISSIONS DES COACHES BIOPAMA POUR LES AIRES PROTÉGÉES}

\section{Cadre:}

- La COMIT est un outil initié et développé par l'Observatoire pour l'Afrique occidentale et centrale pour standardiser la formation des coaches et les actions de renforcement des capacités des gestionnaires des aires protégées, afin d'améliorer les décisions dans la gestion des aires protégées et de réaliser la collecte de données pour l'Observatoire.

- Dans sa conception, la COMIT a été envisagée comme un outil qui serait utilisé par les autres Observatoires BIOPAMA dans les pays ACP, et toute autre utilisation du Formulaire BIOPAMA.

\begin{abstract}
Mission :
Dans le cadre du programme BIOPAMA, la mission confiée aux deux coaches ou binôme est la charge de travail qui leur est assignée pour développer les capacités des gestionnaires des aires protégées et rendre disponible les meilleures informations et données pour améliorer la prise de décision.
\end{abstract}

La mission des coaches est approuvée par leur administration nationale conformément aux accords-cadres institutionnels et aux ressources mises à leur disposition selon un calendrier préétabli.

\section{Étapes des missions :}

La COMIT permet aux coaches de mener de manière efficace leur mission, qui comprend typiquement trois étapes :

1. Initiation et formation des gestionnaires des AP sur le Système d'aide à la décision de l'Observatoire. Les coaches travailleront étroitement avec les institutions nationales, les partenaires locaux concernés et l'Observatoire pour identifier les cibles des interventions.

2. Collecte et analyses des données basées sur le Formulaire BIOPAMA avec les équipes impliquées dans la gestion quotidienne des aires protégées. Les coaches accompagneront les gestionnaires des parcs pour prendre de meilleures décisions et améliorer leur plan de travail annuel dans un processus de gestion adaptative. Dans cette phase, les coaches peuvent apporter également un appui institutionnel en s'assurant que le Système d'aide à la décision est intégré au niveau de la cellule de planification-suivi-évaluation de l'institution nationale.

3. Restitution des missions : il s'agit d'organiser et de conduire un atelier national ou sous-régional pour présenter les résultats des missions de coaching auprès des représentants des administrations nationales et des institutions partenaires clés. Cet atelier permettra de faire surgir des recommandations d'amélioration de la gestion des aires protégées au niveau national, basées sur des données et des informations fiables. II est possible que la restitution se fasse également à l'échelle sousrégionale ou régionale.

\section{Prérequis pour les missions :}

- La sélection des experts pour devenir des coaches d'aires protégées doit être agréée par l'institution nationale et les partenaires. Les experts choisis doivent recevoir une formation et être certifiés compétents pour assurer leur mission de renforcement des capacités des gestionnaires des aires protégées à utiliser le Système d'aide à la décision de l'Observatoire.

- L'administration ou l'agence nationale en charge de la gestion des aires protégées du pays où les coaches résident doit être informée des nouvelles qualifications et rôles des experts formés en tant que coaches de l'Observatoire.

- L'administration ou l'agence nationale en charge de la gestion des aires protégées doit recevoir une lettre officielle de la part des partenaires de l'Observatoire BIOPAMA, et doit autoriser la mise en œuvre de la mission mentionnée préalablement. Elle doit également assurer que toutes les parties prenantes sont présentes, comprennent le processus et ont l'opportunité de s'exprimer

- Une lettre de mission doit définir les objectifs et le déroulement détaillé de la mission. Elle sera établie et signées par un représentant de l'Observatoire.

\section{Nature de la mission du binôme de coaches :}

- Missions de renforcement des capacités conformément à la COMIT. Cela inclut, sans s'y limiter, les réunions d'information et d'initiation au Système d'aide à la décision, l'atelier de formation avec le Formulaire BIOPAMA, les appuis-conseils de l'équipe de 
gestion au niveau du site ou au niveau central sur l'analyse des résultats et l'amélioration de la gestion.

- Les missions de collecte de données à travers le remplissage du Formulaire BIOPAMA. Les coaches sont chargés de diriger l'exercice de remplissage avec l'équipe de gestion du parc. Les coaches assureront le suivi de l'envoi du Formulaire rempli auprès de l'administration centrale et de l'Observatoire, selon un processus convenu entre les parties.

\section{Résumé des rôles des différentes parties prenantes}

\begin{tabular}{|c|c|}
\hline Coach (en binôme) & $\begin{array}{l}\text { - Servir de liens entre l'Observatoire, l'institution nationale, les équipes de } \\
\text { gestion des AP concernées ; } \\
\text { - Assurer la formation et le coaching des équipes de gestion des } \\
\text { aires protégées sur l'utilisation du Système d'aide à la décision de } \\
\text { l'Observatoire. }\end{array}$ \\
\hline Institution nationale & $\begin{array}{l}\text { Donner l'aval et faciliter le déroulement des missions des coaches en } \\
\text { mettant à leur disposition les personnes-ressources concernées et les } \\
\text { moyens disponibles; } \\
\text { - Valider les données pour envoi à l'Observatoire. }\end{array}$ \\
\hline UICN - PACO & $\begin{array}{l}\text { - Établir les accords-cadres de collaboration avec l'institution nationale et les } \\
\text { - Meaches ; } \\
\text { - } \quad \text { Sinancières pour assurer leur mission ; } \\
\text { - Servir de liens entre les pays et les partenaires régionaux et internationaux. }\end{array}$ \\
\hline Observatoire & $\begin{array}{l}\text { - Assurer la mise à disposition des données et informations sur les aires } \\
\text { protégées sur une plateforme facilement accessible. }\end{array}$ \\
\hline Points focaux & $\begin{array}{l}\text { - Octroyer des appuis techniques et stratégiques aux coaches de leur sous- } \\
\text { région pour le bon déroulement de leur mission; } \\
\text { - Animer le réseau des coaches de la sous-région. }\end{array}$ \\
\hline $\begin{array}{l}\text { Personnes-ressources } \\
\text { (consultants) }\end{array}$ & $\begin{array}{l}\text { - Fournir des appuis et conseils techniques aux coaches pour l'utilisation } \\
\text { des outils mis à leur disposition. }\end{array}$ \\
\hline
\end{tabular}

\subsection{L'atelier de formation des gestionnaires}

Cette section vise à aider le coach dans sa casquette de «formateur » et donc dans l'organisation de l'atelier national de formation des gestionnaires d'aires protégées. La maîtrise du Formulaire BIOPAMA n'est pas suffisante pour garantir l'appropriation des principes et des techniques par les participants, elle doit être accompagnée de l'utilisation de techniques de formation qui améliorent l'apprentissage et l'acquisition du savoir, du savoir-faire et des attitudes recherchés.

\subsubsection{Principes généraux pour rendre une formation efficace}

\section{L'andragogie}

(1) La formation est plus efficace lorsqu'elle tient compte des caractéristiques d'apprentissage des adultes, ou " andragogie ». En premier lieu, il est important de créer un climat positif de groupe qui favorise une meilleure assimilation de l'information. Certains experts (Kopylova \& Danilina, 2011) estiment quelaprincipalecomposantepour rendre uneformation efficace est l'environnement d'apprentissage. II est donc crucial de centrer la formation sur l'apprenant et de créer un milieu favorable à l'apprentissage.

Le Guide pour la formation du personnel des aires protégées de l'IUCN (Kopylova \& Danilina, 2011) suggère six points importants à considérer :

- Se focaliser sur les besoins de l'apprenant :

Le premier jour, voire la première heure, de l'atelier est très important pour déterminer l'atmosphère de l'atelier et l'attitude des participants. 
si une évaluation des besoins de formations a été faite préalablement, il est important de la considérer. L'outil d'évaluation des compétences C-EVAL 1 (Partie 4) sera utilisé pour évaluer les compétences des gestionnaires avant la formation. Certains besoins ne sont pas toujours exprimés mais sont importants, par exemple : « l'auto-actualisation » (le besoin d'un développement personnel continu et la mise en œuvre de ses propres idées et compétences), le désir d'apprendre et d'être en avance, le désir d'acceptation dans un groupe, etc.

- Améliorer l'image de soi du participant à la formation, qui commence par le respect de soi et de l'autre. Souvent les professionnels des aires protégées ne sont pas très valorisés par les autres membres de la société, et ont davantage besoin de reconnaissance. En les faisant participer et partager leurs expériences, les participants se sentent valorisés et appréciés.

\section{- Prendre en compte les souhaits des}

\section{Encadré 5 - Quelques principes et techniques d'andragogie}

Assurez-vous que la formation corresponde à ce que les participants veulent. Les adultes sont conscients de leurs besoins et veulent partager la responsabilité de leur propre apprentissage.

Techniques : Présentez à l'avance le programme de la formation, discutez-en en début de la formation.

Assurez-vous de combler des besoins immédiats. Les participants seront plus motivés si la formation cherche à combler leurs besoins immédiats.

Techniques : Interrogez-les sur leurs attentes, soit avant de venir à la formation, soit le premier jour de la formation. Adapter le programme et les activités en conséquence (s'ils entrent dans les objectifs de l'atelier).

Encouragez la participation active de tous les participants. Une formation qui repose sur l'expérience et l'action est plus durable et efficace.

Techniques : Diversifiez les techniques de formation : jeux de rôles, débats, exercices pratiques, travail en binôme, en groupe, mini-projets etc. Soyez créatifs, mais clairs !

Faites reposer la formation sur l'expérience. L'apprentissage le plus efficace repose sur l'échange d'expériences vécues.

Techniques : Laissez le temps aux participants de partager ou d'utiliser leur vécu, afin de servir d'exemples ou d'études de cas.

Accompagnez la réflexion en donnant aux participants l'opportunité de réfléchir sur le passé et d'en tirer les enseignements, et sur le moment de la formation.

Techniques : Planifiez des activités qui permettront aux participants de revoir ce qu'ils ont fait. Favorisez les réflexions sur les problèmes, et les stratégies utilisées pour les résoudre.

Créez une atmosphère saine en vous assurant que tout le monde est à l'aise. Une atmosphère saine, collaborative et dans la bonne humeur favorise l'apprentissage et la participation.

Techniques : Fournissez suffisamment de temps et d'informations sur l'atelier, incluant les dispositions logistiques. Brisez la glace et détendez l'atmosphère en début et en cours d'atelier. Soyez sympathiques !

Assurez-vous que le cadre est agréable en veillant à ce que les participants soient bien nourris, bien reposés et en bonne santé pour apprendre avec le maximum de rendement.

Techniques : Sélectionnez avec soin le lieu de formation. Prévoyez une collation pendant les pauses. Prenez en compte les plaintes.

Encouragez les réactions en permettant aux participants de faire des commentaires, positifs ou négatifs. Techniques : Encouragez les participants à émettre leurs points de vue, n'émettez pas de jugements sur les commentaires.

Source : Stone (1998) Quel est votre rôle ? La formation et son incidence sur l'organisation. Guide à l'intention des responsables de la formation à la gestion des aires protégées, Parcs 6-2 à 4 


\begin{tabular}{|l|l|}
\hline $\begin{array}{l}\text { Formation centrée sur le formateur } \\
\begin{array}{l}\text { Le savoir est transmis du coach-formateur au } \\
\text { participant. }\end{array}\end{array}$ & $\begin{array}{l}\text { Formation centrée sur l'apprenant } \\
\text { leurs connaissances par la recherche et la collecte } \\
\text { d'informations, la pensée critique, la résolution de } \\
\text { problèmes, la recherche et la communication. }\end{array}$ \\
\hline $\begin{array}{l}\text { Les participants reçoivent des informations } \\
\text { passivement. }\end{array}$ & $\begin{array}{l}\text { Les apprenants participent activement à la } \\
\text { formation. }\end{array}$ \\
\hline $\begin{array}{l}\text { La connaissance est acquise indépendamment de } \\
\text { l'application au monde réel. }\end{array}$ & $\begin{array}{l}\text { L'application effective des connaissances aux } \\
\text { problèmes du monde réel est mise en avant. }\end{array}$ \\
\hline $\begin{array}{l}\text { Le coach-formateur est le premier fournisseur } \\
\text { d'information et le principal évaluateur. }\end{array}$ & $\begin{array}{l}\text { Le coach-formateur est l'animateur et le formateur. } \\
\text { L'enseignant évalue l'apprentissage avec les } \\
\text { étudiants. }\end{array}$ \\
\hline $\begin{array}{l}\text { Les bonnes réponses sont mises en exergue. } \\
\text { apprenants. }\end{array}$ & $\begin{array}{l}\text { La formation permet de discuter et d'examiner les } \\
\text { réponses et d'apprendre à partir des erreurs. }\end{array}$ \\
\hline $\begin{array}{l}\text { Culture de formation individualiste et compétitive. } \\
\text { Culture de coopération, de collaboration et de }\end{array}$ & \begin{tabular}{l} 
soutien mutuel. \\
\hline
\end{tabular} \\
\hline
\end{tabular}

Adapté de : Huba, M.E. and J.E Freed. 2000. Pages 8-15, 66-67 in Learner-centered assessment on college campuses: shifting the focus from teaching to learning. Allyn and Bacon, Boston, Massachusetts, États-Unis.

participants : de manière générale, les formations relatives aux aires protégées sont focalisées sur le contenu pour satisfaire les ambitions professionnelles. II est cependant important de considérer ce que les participants aiment faire, et de rendre la formation agréable. Le responsable de formation doit être attentif et adapter le programme en conséquence.

- Faciliter l'avancement professionnel en démontrant clairement les liens entre le contenu de la formation et leur travail au quotidien. Par exemple, le coach-formateur peut donner un exemple du type de résultats à la fin du remplissage du Formulaire pour susciter l'intérêt du Conservateur.

- Favoriser un milieu favorable à l'apprentissage : l'encadré suivant donne plusieurs éléments pour rendre la formation agréable, propice à l'apprentissage et à la collaboration.

- Créer des situations informelles pour consolider le groupe et favoriser les échanges : il est fortement recommandé de rendre la formation agréable, sympathique avec des moments de loisirs et de détente.

\section{L'apprentissage actif et centré sur l'apprenant}

Les techniques d'enseignement ou de formation dites « actives » et " centrées sur l'apprenant » permettent d'intégrer l'apprenant dans le processus d'apprentissage. Voici quelques caractéristiques générales d'une formation active et centrée sur l'apprenant :

- Les apprenants sont impliqués au-delà de la simple écoute, ils participent et sont engagés dans des activités (ex : lecture, discussion, rédaction) ;

- L'accent est davantage mis sur le renforcement des capacités plutôt que sur la transmission d'informations ;

- Les apprenants sont impliqués dans des réflexions plus élevées (analyse, synthèse, évaluation) ;

- La diversité des techniques de formation utilisées permet de toucher différents styles d'apprenants ;

- L'accent est mis sur l'étude des valeurs et attitudes des apprenants.

En d'autres termes, on pourrait définir l'enseignement actif comme tout ce qui implique les apprenants à agir et à réfléchir sur ce qu'ils font. 


\begin{tabular}{|c|c|c|}
\hline Objectifs & \multicolumn{2}{|l|}{ Techniques } \\
\hline Comprendre une notion & $\begin{array}{l}\text { Cours } \\
\text { Visualisation d'une diapositives/film } \\
\text { Dramatisation } \\
\text { Débat, Dialogue } \\
\text { Exposition } \\
\text { Questions-réponses } \\
\text { Étude de cas }\end{array}$ & $\begin{array}{l}\text { Entretien (en binôme ou en petit } \\
\text { groupe) } \\
\text { Visite de sites } \\
\text { Démonstration, essai } \\
\text { Colloque, Forum }\end{array}$ \\
\hline Maîtriser une technique & $\begin{array}{l}\text { Démonstration, essais, } \\
\text { Simulation } \\
\text { Exercice pratique }\end{array}$ & $\begin{array}{l}\text { Démonstration et mise en pratique } \\
\text { Démonstration silencieuse } \\
\text { Stages }\end{array}$ \\
\hline $\begin{array}{l}\text { Acquisition d'attitudes et } \\
\text { de valeurs }\end{array}$ & $\begin{array}{l}\text { Discussion et échange sur l'expérience de chacun } \\
\text { Jeu de rôles } \\
\text { Étude de cas } \\
\text { Exercice non verbal } \\
\text { Groupe d'écoute }\end{array}$ & $\begin{array}{l}\text { Débriefing, pratique réflexive sur } \\
\text { une situation } \\
\text { Visite de site } \\
\text { Exercice de clarification des valeurs }\end{array}$ \\
\hline $\begin{array}{l}\text { Faire participer tout le } \\
\text { groupe }\end{array}$ & $\begin{array}{l}\text { Discussion ouverte } \\
\text { Carte de réponse }\end{array}$ & $\begin{array}{l}\text { Enquête, sondage } \\
\text { Exercices en plénière }\end{array}$ \\
\hline $\begin{array}{l}\text { Favoriser l'apprentissage } \\
\text { collaboratif }\end{array}$ & $\begin{array}{l}\text { Travail en groupe } \\
\text { Travail en binôme }\end{array}$ & Projet de groupe \\
\hline
\end{tabular}

\section{Les démarches proposées sont à adapter selon le contexte local}

Pour qu'une technique soit efficace : Soyez bien préparé !

Considérez d'abord votre objectif en tant que formateur pour choisir la technique appropriée.

Préparez à l'avance par écrit toutes les étapes de la technique. Une technique peut comporter plusieurs étapes. Prévoyez aussi les outils et équipements nécessaires.

Énoncez clairement les instructions lorsque tout le monde a votre attention. Par exemple, il est préférable de donner les instructions avant de départager les groupes.

Plus c'est tôt, mieux c'est!

\section{Encadré 6 - Liste de contrôle des tâches et calendrier de préparation}

Au moins 1 mois à l'avance :

- Recontacter l'institution nationale et les mêmes personnes rencontrées lors de la première séance de travail (Partie 1.1)

- Rédaction du cahier des charges de l'atelier

- Communication aux partenaires

- Préparation préliminaire du calendrier des missions sur le terrain

- Identification/confirmation du lieu de l'atelier : hébergement, salle de formation, restauration, hébergement des participants

- Préparation et soumission du budget

Au moins 2 semaines à l'avance :

- Réception des noms des participants et confirmation

- Élaboration du plan détaillé de formation avec répartition claire des rôles pour la préparation et la conduite de la formation

Au moins 1 semaine à l'avance, obtenir du gestionnaire et de l'institution les informations suivantes :

- La liste définitive des participants

- Le programme final de la formation, le partage des responsabilités

- Les détails logistiques

1 semaine après la formation :

Rédaction d'une version provisoire du rapport de la formation

1 mois après la mission :

Soumission de la version définitive du rapport technique et financier 


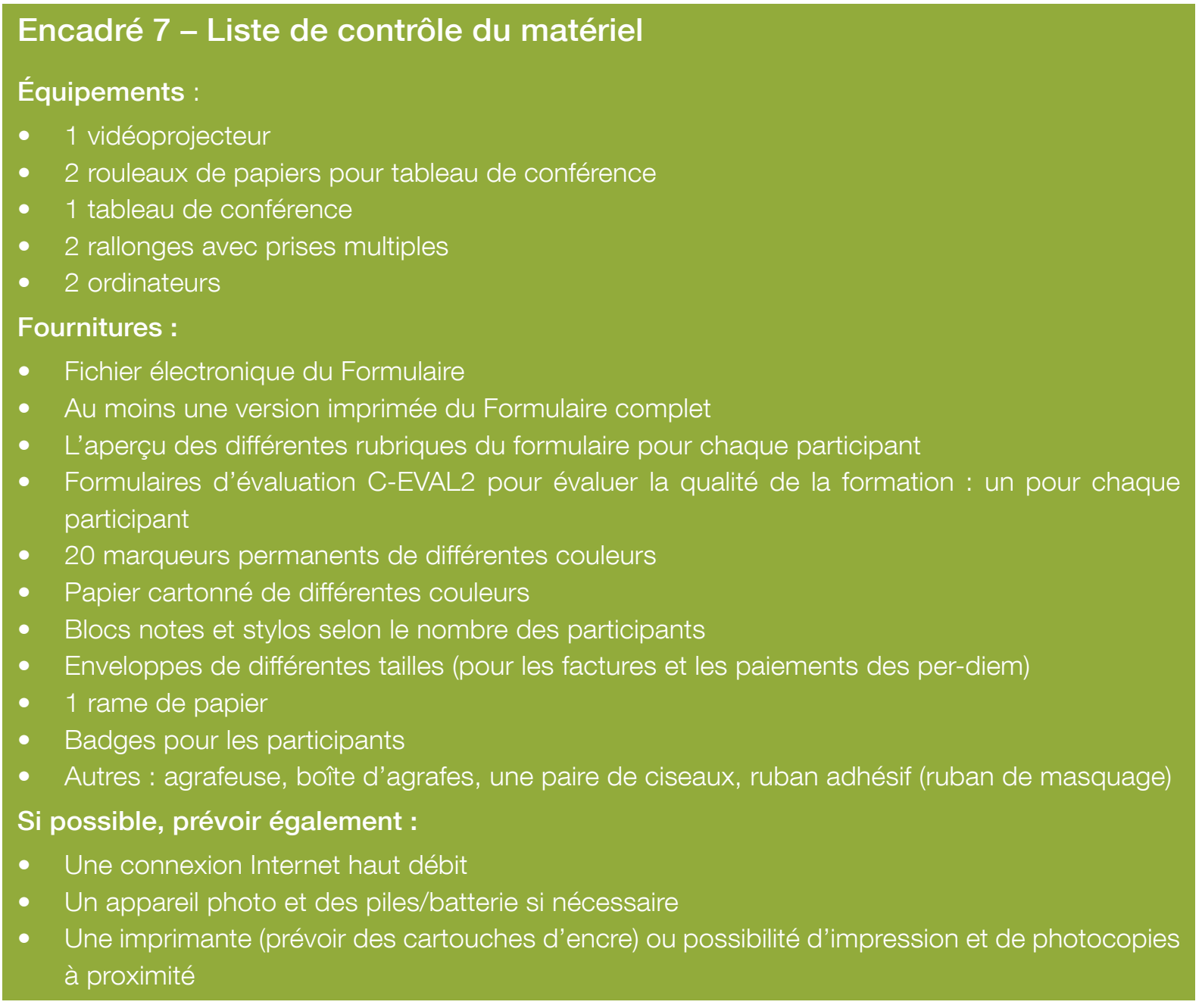

\subsubsection{Quelques techniques utilisables dans la formation}

On trouve sur Internet ou dans des rapports des milliers de suggestions de techniques de formation visant à rendre l'apprenant acteur de son propre apprentissage. Une technique peut être utilisée de plusieurs manières selon les situations (nombre d'apprenants, espace et temps disponibles, objectifs souhaités). Les spécialistes en formation ont souvent attribué des noms à ces techniques pour les différencier les unes des autres. Les suggestions suivantes sont données à titre indicatif.

\subsubsection{Procédures d'organisation}

L'organisation de la formation des gestionnaires d'aires protégées d'un pays est un projet collaboratif étroitement lié avec l'institution de tutelle de ces AP. Les démarches préliminaires (voir partie 1 du document) ont permis d'établir le calendrier des interventions et les procédures d'organisations (notamment les dates, le lieu et le nom des participants ou AP concernées). Le binôme de coaches et un ou deux représentants de l'institution de tutelle formeront le comité d'organisation de la formation.

Les encadrés 6 et 7 sont établis pour servir de liste de contrôle (checklist).

\subsubsection{Déroulement de l'atelier national d'appropriation de l'outil}

L'atelier d'appropriation de l'outil au niveau national a pour but de démontrer l'utilité du Formulaire BIOPAMA, de former les gestionnaires sur le formulaire et de poser les fondements de la campagne de renforcement des capacités. À la fin de cet atelier, les administrateurs et les gestionnaires des AP concernées auront compris l'importance et le fonctionnement de l'outil, et auront développé ensemble un plan d'action spécifique pour organiser les missions d'utilisation de l'outil. Le canevas suivant est proposé pour guider l'élaboration du programme détaillé de cet atelier de formation par le binôme de coaches. 


\section{Encadré 8 - Modèle de programme de l'atelier national}

Jour 1:

Accueil des participants. Présentation des participants et des objectifs de l'atelier et des messages clés. Règles de base de l'atelier

\section{SESSION 1 : Présentation générale}

- Le BIOPAMA et l'Observatoire régional

- Formulaire BIOPAMA : utilités et valeurs ajoutées

SESSION 2 : La gestion et la gouvernance des aires protégées

- Principes généraux

- Contexte de l'intervention, efficacité de la gestion et qualité de la gouvernance

- Cycle de gestion

- Généralités sur l'évaluation de l'efficacité de la gestion

Jour 2:

\section{SESSION 3 : Présentation du Formulaire}

- Contexte de l'intervention

- Efficacité de la gestion : Contexte de la gestion, planification, apports, processus, résultats et effets/ impacts

SESSION 4 : Exercices sur des éléments du Formulaire : Contexte de l'intervention

- Identification des valeurs et importance d'une AP

- Analyse des menaces

- Identification des effets du changement climatique et des services écosystémiques

- Analyse à l'aide des éléments de visualisation

Jour 3 :

SESSION 5 : Exercices sur des éléments du Formulaire : Efficacité de la gestion

- Contexte de la gestion, planification, apports, processus, résultats et effets/impacts

- Analyse à l'aide des éléments de visualisation

- Formulation des objectifs, indicateurs et valeurs de référence (benchmarks)

- Notification du contexte de l'intervention, de l'efficacité de la gestion et des propositions d'intervention

Jour 4 :

SESSION 6 : Étude de cas : Exemple en salle ou au cours d'une brève visite sur terrain

- Contexte de l'intervention

- Efficacité de la gestion

- Analyse à l'aide des éléments de visualisation

- Formulation des objectifs, indicateurs et valeurs de référence (benchmarks)

Jour 5 :

SESSION 7 : Préparation de l'étape de terrain

- Organisation logistique

- Collecte et analyse des données existantes

- Pré-remplissage du Formulaire

SESSION 8 : Analyses et restitution

- Synthèse des informations, résultats, analyses et propositions

- Présentation et discussion

- Formulation des étapes à venir des interventions et du coaching 


\subsection{Le suivi-accompagnement}

\subsubsection{Démarches d'accompagnement}

L'accompagnement est un processus étalé sur le temps. C'est au coach de maintenir les contacts et l'intérêt des personnes et des institutions dans le processus à travers un compte-rendu régulier, des échanges par emails, voire des visites. Les démarches suivantes donnent les étapes principales.

\subsubsection{La mission sur site}

La mission du binôme de coaches dans l'aire protégée est une étape importante de l'accompagnement après l'atelier de formation nationale. Si celui-ci avait permis des premières planifications entre l'équipe de gestion et les coaches, la mission dans l'aire protégée permettra d'entrer en détail dans l'évaluation de l'efficacité de la gestion du site en question. La section précédente permet de préparer au mieux cette visite sur le terrain.

\section{TRÈS IMPORTANT : Le pré-remplissage doit être effectué avant la visite sur site.}

Deux cas de figure peuvent se présenter, selon les contraintes d'organisation logistique et de programmation :

1. La mission consiste à faire une visite de terrain pour mieux appréhender les réalités sur le terrain. II s'agit alors d'une courte mission (1-2 jours maximum) pour les

\section{Encadré 9 - Protocole de mission}

Au moins 1 mois à l'avance :

- Collaborer avec le gestionnaire formé pour confirmer sa disponibilité sur les dates fixées lors de la formation. Fixer de nouvelles dates si nécessaire.

- Recontacter l'institution nationale (copie au gestionnaire déjà formé) en charge de l'AP pour prévenir de la mission sur terrain. De préférence, contacter les mêmes personnes rencontrées lors de la première séance de travail (Partie 1.1) :

- Informer et valider les dates fixées

- Obtenir une lettre d'introduction ou lettre d'invitation officielle

- Mobiliser les bonnes personnes : l'équipe de gestion de l'AP et quelques représentants de la cellule suivi-évaluation ou la direction technique

- Proposer un programme de la mission : dates terrain et journées bloquées d'analyse

- Organiser la logistique : trajet, matériels et équipements

Au moins 2 semaines à l'avance :

- Obtenir du gestionnaire le plan de gestion de l'AP ou des AP. Répartir les tâches de préremplissage entre le binôme de coach

- Consulter d'autres documents sur l'AP

- Commencer le pré-remplissage du Formulaire de l'AP

Au moins 1 semaine à l'avance, obtenir du gestionnaire et de l'institution les informations suivantes :

- La liste définitive des participants. Y a-t-il des personnes qui assisteront uniquement à la restitution ? ex : les partenaires locaux

- Le programme final de la mission, le partage des responsabilités

- Les détails logistiques : départ pour le terrain, pendant la mission, retour.

1 semaine après la mission :

Rédaction d'une version provisoire du rapport de mission comprenant les premières recommandations.

1 mois après la mission :

Soumission de la version définitive du rapport technique et du rapport financier 


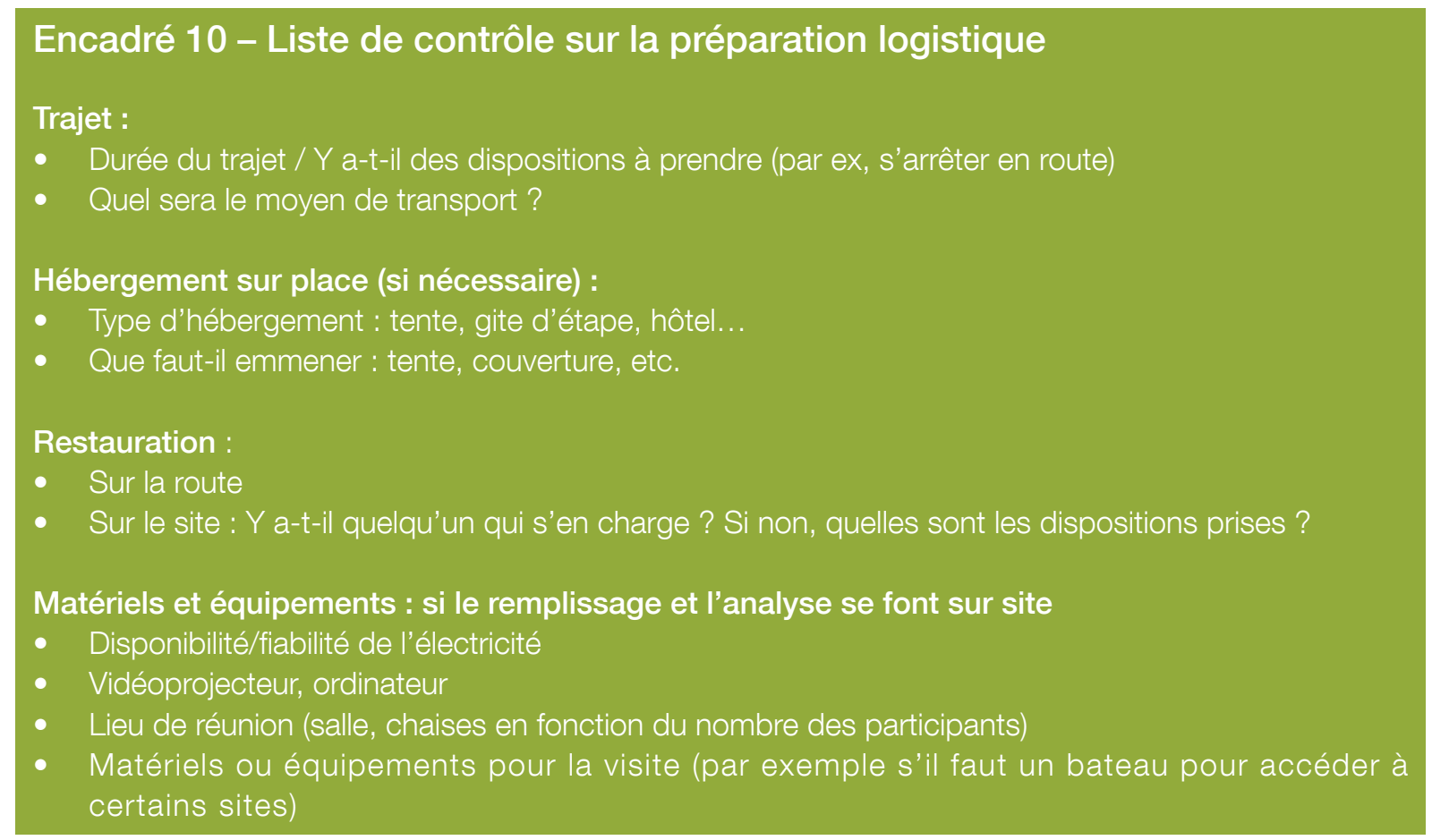

coaches, sans séance de travail. L'utilisation du Système d'information comme aide à la décision se fera dans un autre endroit, tel que les bureaux de l'agence nationale, en ville.

2. La mission comprend à la fois une visite des lieux et une séance de travail pour l'utilisation du Système d'information. Les coaches doivent alors s'organiser conformément à la partie 3 du présent document.

À prévoir pour la visite des lieux :

- Une carte de l'aire protégée, avec plan d'aménagement

- Une carte de la région

- Le Formulaire offline pré-rempli, avec une liste de questions pour le gestionnaire.

\subsubsection{L'accompagnement après un premier remplissage}

Après la visite sur le terrain et la séance de travail pour remplir le Formulaire BIOPAMA, le coach doit maintenir un contact régulier avec l'équipe de gestion, soit à distance (par email), soit dans le cas où le coach est rattaché à l'institution nationale, par de courtes visites ou des petites réunions de travail. L'objectif pour le coach est de pouvoir :

- Fournir des remontées d'informations techniques face aux questions éventuelles du gestionnaire sur le Formulaire BIOPAMA.
En effet, du temps lui est donné pour étudier le Formulaire ;

- Fournir un support dans l'élaboration des propositions d'amélioration de l'efficacité de la gestion ;

- Préparer les séances de présentation au niveau de l'institution nationale ou auprès de partenaires techniques et financiers locaux ;

- Aider à la collecte des Formulaires remplis ;

- Encourager l'intégration progressive du remplissage du Formulaire BIOPAMA dans le plan de travail annuel, et l'utilisation des informations du Système d'information (Observatoire) pour la prise de décision.

Au niveau de l'institution nationale, le coach est appelé à travailler étroitement avec la/les direction(s) chargée(s) de la planification et du suivi-évaluation notamment pour :

- Contribuer à améliorer le flux d'informations entre l'organe central et les aires protégées impliquées dans le Programme BIOPAMA ;

- Contribuer à une analyse du réseau ou système d'aires protégées grâce à la comparaison des différents résultats reçus ;

- Améliorer les mécanismes de prise de décision, de planification et de suiviévaluation grâce à l'intégration progressive de l'utilisation du Système d'information (Observatoire) ;

- Préparer des restitutions auprès de la direction générale et des partenaires nationaux et internationaux. 


\subsection{Planification et reporting}

La planification des activités du coach se fera en concertation avec son binôme, l'institution nationale en charge des aires protégées, le Programme BIOPAMA ainsi que toute autre institution nationale ou sous-régionale impliquée.

Le binôme de coaches devra remettre les rapports de ses interventions auprès du BIOPAMA et des entités concernées. Les rapports principaux à soumettre sont le rapport de l'atelier de formation nationale avec les gestionnaires des aires protégées, et le rapport des missions de remplissage du Formulaire et d'accompagnement au niveau des aires protégées. Le coach doit également participer à l'élaboration du rapport sur les résultats obtenus et les propositions d'amélioration de gestion des aires protégées établi par le Conservateur.

\section{Des modèles de rapport sont disponibles dans la clé USB.}

\subsection{Forum des coaches}

La campagne de renforcement des capacités du Programme BIOPAMA ne se limite pas uniquement à une interaction entre le coach et le coaché (équipe de gestion d'une aire protégée et son institution de tutelle). Elle vise également à renforcer l'expertise des coaches dans l'évaluation de l'efficacité de la gestion et l'accompagnement à la prise de décisions. La création d'une communauté de pratique de coaching des aires protégées est un des résultats attendus de la campagne.
Les coaches sont encouragés à utiliser le forum sur le site du Système régional d'information de référence du BIOPAMA http://rris. biopama.org/forum pour :

- Partager leurs expériences de coaching ;

- Poser des questions et recevoir des remontées d'informations des coaches et des experts ;

- Réfléchir sur des situations qu'ils ont rencontré grâce aux échanges et des remontées d'information avec les autres coaches.

II suffit de s'inscrire pour avoir accès au forum. Chaque coach pourra ainsi publier des commentaires ou des questions, et recevoir les réponses.

\subsection{Ressources pour le coach}

\section{Liens web et bibliographie}

Sur le coaching :

Association européenne de coaching www.aecoaching.eu

Société française de coaching www.sfcoach.org

International coach federation www.coachfederation.org

Sur l'organisation de formation et la pédagogie active Stone R. (1998) Quel est votre rôle ? La formation et son incidence sur l'organisation. Guide à l'intention des responsables de la formation à la gestion des aires protégées. Série Biodiversité africaine $n^{\circ} 5$. Washington, DC : Biodiversity Support Program

Kopylova, S.L. and Danilina, N.R. (Editors) (2011). Protected Area Staff Training: Guidelines for Planning and Management. Gland, Suisse : UICN.
Domroese M.C. Fialkowski C. et E.J. Sterling (2005). Nouvelles techniques pour enseigner la conservation de la biodiversité. Module du Réseau des Éducateurs et Professionnels de la Conservation. Synthèse. 40 pp

Mériaux S. (FIBA) et Staub F. (Biodiversité Conseil), 2014, Préparer, animer et évaluer une formation guide pratique, p.24 http://www.biodiv-conseil.fr/ PDF/Guide_formation.pdf

\section{Fichiers dans la clé USB}

4-2 No. 17 - Protected area staff training

4-3 Meriaux \& Staub Guide_formation

0-7 Rapport Mission Parc

0-8 Liste participants Annexe 1

0-9 Rapport financier mission

\section{En cas de difficultés}

Coordination de la campagne:

Domoina Rakotobe

domoina.rakotobe@ext.iucn.org 


\title{
PARTIE 3
}

\section{Comment utiliser le système d'aide à la décision?}

\author{
QUAND UTILISER CETTE PARTIE : \\ Pendant l'atelier de formation des gestionnaires et \\ acteurs de la conservation. \\ Pendant la réunion de travail avec l'équipe de \\ gestion. \\ COMMENT : Cette partie est composée des \\ définitions de concepts clés et d'instructions pour le \\ remplissage manuel et l'interprétation des résultats. \\ Le coach doit maîtriser les concepts de bases \\ avant d'entamer le remplissage du Formulaire de \\ l'Observatoire.
}

\begin{abstract}
DURÉE : Le remplissage du Formulaire dure entre 16 et 32 heures de réunion de travail. L'accompagnement de l'équipe de gestion peut prendre jusqu'à six mois, avec des interventions plus ou moins fréquentes.
\end{abstract}

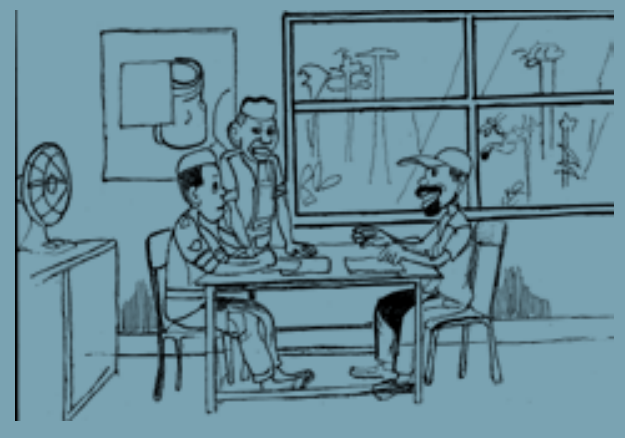

\subsection{Présentation du système d'aide à la décision}

Attention ! Cette partie se focalise sur les questions de compréhension des indicateurs à renseigner dans le Formulaire et n'abordera pas les aspects liés à la manipulation informatique (voir partie 4).
L'Observatoire des aires protégées et de la biodiversité est organisé en un Système d'aide à la décision (Decision Support System (DSS)). Le Formulaire BIOPAMA permet de collecter une large gamme de données et de les présenter de façon à faciliter l'interprétation d'informations pour la prise de décision dans une approche de gestion adaptative.

Encadré 11 - La gestion adaptative, une approche structurée pour la prise de décision

La gestion adaptative est une approche systématique pour améliorer la gestion des ressources par l'apprentissage des impacts de la gestion. Elle est rarement mise en œuvre, même si de nombreux documents de planification des ressources la recommandent et de nombreux gestionnaires de ressources s'y réfèrent. Beaucoup considèrent qu'en suivant des activités et en faisant à l'occasion des changements, ils font une gestion adaptative. Contrairement à cette croyance répandue, la gestion adaptative est beaucoup plus que le suivi et le changement de l'orientation de la gestion pour faire face à des stratégies ratées. En fait, une telle tactique pourrait plutôt être non-adaptative. Une approche adaptative consiste à explorer d'autres moyens d'atteindre les objectifs de la gestion, de prédire les impacts des alternatives, en se basant sur l'état actuel des connaissances, la mise en œuvre d'une ou plusieurs de ces alternatives, le suivi des impacts des actions de gestion, et l'utilisation des résultats pour mettre à jour et ajuster les mesures de gestion. La gestion adaptative se concentre sur l'apprentissage et l'adaptation, grâce à des partenariats avec les gestionnaires, les scientifiques et d'autres intervenants qui apprennent ensemble comment créer et maintenir des systèmes de ressources durables.

Tiré de : DOI/AMWG (2012) Adaptive Management Application Guides, chapitre 1.

Consulté sur le site http://www.doi.gov/ppa/Adaptive-Management.cfm 


\subsubsection{Présentation du Formulaire BIOPAMA}

\section{Nota Bene \\ Le présent guide d'utilisation du Formulaire BIOPAMA est destiné à la première version de l'outil d'analyse du contexte de l'intervention, d'évaluation de l'efficacité de la gestion des aires protégées en Afrique centrale et occidentale.}

\section{Organisation du Formulaire}

Pour rappel, le Formulaire s'inspire de plusieurs sources et instruments utilisés pour l'évaluation de l'efficacité de la gestion. II est constitué de trois parties :

- L'état du contexte de l'intervention ;

- L'évaluation de l'efficacité de la gestion ;

- La visualisation de l'évaluation des éléments du cycle de gestion comme aide à la décision.

\section{A. L'état du contexte de l'intervention}

Les éléments de l'état du contexte de l'intervention permettent d'organiser et de synthétiser les informations de base sur l'aire protégée. Le résultat est :

- La disponibilité ou la mise à jour d'une monographie du parc pour avoir les informations importantes et essentielles du contexte de l'intervention de la conservation de l'aire protégée ;

- La détermination des éléments à utiliser dans l'analyse de l'efficacité de la gestion de l'aire protégée.

\section{B. L'évaluation de l'efficacité de la gestion}

Comme pour la majorité des outils d'évaluation de l'efficacité de la gestion, le Formulaire organise les éléments de l'analyse sur la base du cycle de gestion des aires protégées (UICN, Prof. Hockings et collègues). L'évaluation de l'efficacité de la gestion est divisée en six éléments :

1. Contexte de la gestion

2. Planification

3. Intrants

4. Processus

5. Résultats (Outputs)

6. Effet/Impact (Outcomes)

Sur la base des indications de l'étude Management effectiveness evaluation in protected areas - a global study - Second edition - 2010, et considérant son importance dans l'analyse et sa complexité, le Processus est subdivisé en six sous-éléments :

1. Systèmes et processus de gestion interne

2. Protection/Gestion

3. Relations avec les parties prenantes

4. Gestion du tourisme

5. Suivi et recherche

6. Gestion des effets du changement climatique et des services écosystémiques.

\section{La visualisation de l'évaluation des éléments du cycle de gestion comme aide à la décision}

La représentation à l'aide de graphiques permet d'afficher et de résumer les résultats de l'évaluation de l'efficacité de la gestion de l'aire protégée. Cependant, la visualisation ne donne qu'un affichage des résultats des analyses et de l'évaluation. Les outils de visualisation doivent être utilisés seulement comme support et facilitation de la réflexion à conduire pour la prise de décision.

\section{Explication de l'utilisation du Formulaire}

Ce Formulaire a été établi en distinguant différentes sections, chaque section permettant de renseigner un élément spécifique de l'état du contexte de l'intervention et de l'efficacité de la gestion.

Le Formulaire permet de déterminer les objectifs, les résultats à proposer comme priorités ou les lignes directrices pour améliorer l'efficacité de la gestion. L'outil favorise ainsi la réflexion pour passer d'un état d'intervention vers un état favorable aux valeurs de conservation ou de gestion durable des ressources naturelles de l'aire protégée.

Pour permettre des évaluations plus détaillées, à même de mettre en évidence les évolutions dans la gestion, le Formulaire est organisé en série de questions. Les réponses doivent être fournies en utilisant des barèmes simplifiés selon une échelle $0-1-2-3$ ou, dans certains cas, des valeurs positives $(+1 /+2 /+3)$ pour évaluer des processus d'amélioration, ou négatives pour évaluer des processus de dégradation (-1/ -2/ -3) d'un phénomène. II est important de préciser que les valeurs affichées ne sont pas des estimations absolues, mais sont représentatives de l'évaluation, permettant toutefois de faciliter la représentation des analyses et des considérations formulées pour constituer une aide à la décision (voir point suivant). 


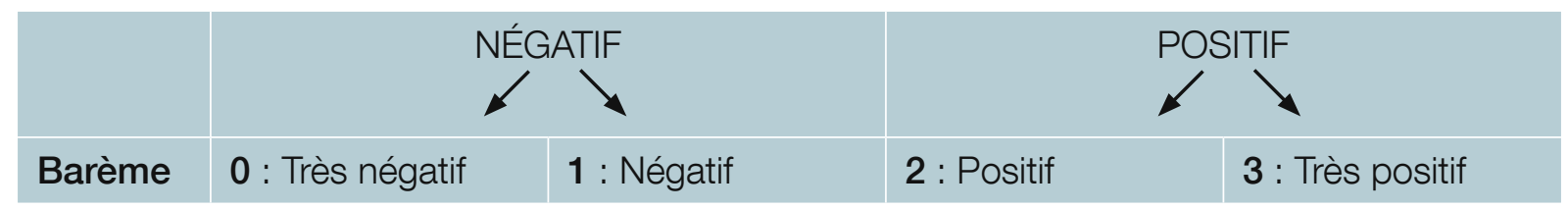

Les évaluations formulées selon les barèmes 0 $-1-2-3$ ou les évaluations de la tendance du phénomène sont estimées selon une échelle de 0-100 sur la base de critères et formules statistiques. Enfin les résultats sont représentés à l'aide de graphiques permettant une visualisation des analyses d'aide à la décision.

\section{Paramétrage du Formulaire}

La détermination de l'état du contexte de l'intervention et l'évaluation de l'efficacité de la gestion, orientés vers l'identification des priorités de gestion et de gouvernance des aires protégées, reposent sur la collecte d'informations ciblées. L'exigence de disposer d'une analyse spécialisée impose un certain paramétrage du Formulaire par rapport à chaque aire protégée et pays. Pour exécuteruneanalyse ciblée, ilest donc indispensable d'effectuer le paramétrage spécifique du Formulaire BIOPAMA à l'occasion de la première évaluation d'une aire protégée. Au moment de l'évaluation de la première aire protégée dans un pays, il est recommandé, en plus du paramétrage spécifique à l'aire protégée, d'insérer dans le Formulaire les critères communs au système de conservation du pays. La configuration "pays " du Formulaire permettra d'éviter la répétition de la recherche de certaines variables, facilitera la réalisation de l'évaluation et permettra I'harmonisation des critères d'analyse dans toutes les autres aires protégées du même pays.
Cependant, chaque élément à analyser présente des variables qui peuvent ne pas correspondre à une aire protégée spécifique, ou bien il peut être nécessaire pour l'AP en question d'insérer des éléments complémentaires. En raison de ce dernier aspect, chaque élément d'analyse présente la possibilité d'intégrer les indications de base en soutien de l'analyse. En complément des indications précédentes, chaque élément ou paramètre d'analyse est accompagné d'un espace permettant la formulation d'observations ou de précisions sur les informations ou l'analyse portées.

Cet exercice d'insertion des éléments de base du système de conservation ou de l'aire protégée dans le Formulaire ne sera plus répété à l'avenir, mais seulement affiné en fonction de l'évolution du processus de gestion, le cas échéant.

Cet exercice de paramétrage assure la spécificité de l'outil d'évaluation par rapport à la spécificité de chaque aire protégée, mais n'empêche pas l'utilisation des résultats des analyses pour la prise de décision au niveau supérieur (national et régional) en raison de l'utilisation de la même structuration des informations recueillies. De plus au niveau national et régional, il est possible de fixer des résultats et effets/impacts communs et de fixer en conséquence des indicateurs et de valeurs de référence (benchmarks) à atteindre pour les réseaux de conservation. L'analyse et l'évaluation de l'évolution des efforts de conservation pourront être suivies à l'aide du Formulaire. 


\section{Les différentes rubriques du Formulaire}

\section{CONTEXTE DE L'INTERVENTION}

CTX.1. Informations générales sur l'aire protégée

CTX.2. Superficies, limites et indices de forme, niveau de contrôle de l'AP

CTX.3. Ressources humaines, financières et matérielles de l'aire protégée

CTX.4. Eléments clés des aires protégées terrestres et marines : i) espèces phares, menacées, endémiques ; ii) habitats ; iii) changements dans la couverture des sols et iv) gestion des ressources

CTX.5. Pressions et menaces sur l'aire protégée

CTX.6. Changement climatique et conservation

CTX.7 Services écosystémiques et dépendance des communautés de l'aire protégée envers ces services

\section{EFFICACITÉ DE LA GESTION}

Contexte de la gestion

\begin{tabular}{|l|l|}
\hline C1 & Valeur et importance \\
\hline C1.1 & Gouvernance \\
\hline C1.2 & Classifications \\
\hline
\end{tabular}

C1.3 Espèces clés

C1.4 Habitats

C1.5 Changement climatique

C1.6 Services écosystémiques

C2 Contrainte ou soutien par I'environnement politique et civil externe

C3 Menaces

C4 Détermination des objectifs-cibles de conservation et des indicateurs relatifs au contexte de la gestion de l'aire protégée

Planification

\begin{tabular}{|l|l|}
\hline P1 & Adéquation des dispositions législatives et réglementaires \\
\hline P2 & Conception et configuration de l'aire protégée \\
\hline P3 & Démarcation de l'aire protégée \\
\hline P4 & Plan de gestion \\
\hline P5 & Plan de travail \\
\hline P6 & Objectifs de l'aire protégée \\
\hline Intrants & \multicolumn{2}{|l|}{} \\
\hline I1 & Informations de base \\
\hline I2 & Personnel \\
\hline I3 & Ressources financières \\
\hline I4 & Sécurisation du budget \\
\hline I5 & Infrastructures, équipements et installations \\
\hline Processus \\
\hline PR1 & Capacités et formation du personnel \\
\hline PR2 & Politiques et procédures de gestion des ressources humaines \\
\hline PR3 & Systèmes et processus de gestion des ressources humaines \\
\hline PR4 & Administration et leadership interne \\
\hline PR5 & Gestion administrative/comptable et financière \\
\hline PR6 & Entretien des infrastructures, des équipements et des installations \\
\hline
\end{tabular}




\begin{tabular}{|c|c|c|}
\hline PR7 & Gestion des valeurs et des aspects importants de l'aire protégée & \multirow{4}{*}{ Protection et gestion } \\
\hline PR8 & $\begin{array}{l}\text { Systèmes de protection des valeurs et des aspects importants de l'aire } \\
\text { protégée }\end{array}$ & \\
\hline PR9 & Contrôle de l'aire protégée & \\
\hline PR10 & Application des lois & \\
\hline PR11 & $\begin{array}{l}\text { Implication des communautés locales, des ayants droit et des parties } \\
\text { prenantes }\end{array}$ & \multirow{3}{*}{$\begin{array}{l}\text { Relations avec les } \\
\text { parties prenantes }\end{array}$} \\
\hline PR12 & Avantages/assistance appropriés au profit des collectivités & \\
\hline PR13 & Relations avec les parties prenantes & \\
\hline PR14 & Gestion des visiteurs & \multirow{2}{*}{ Gestion du tourisme } \\
\hline PR15 & Visiteurs et impacts & \\
\hline PR16 & $\begin{array}{l}\text { Systèmes de suivi des valeurs et des aspects importants de l'aire } \\
\text { protégée }\end{array}$ & \multirow{2}{*}{ Suivi et recherche } \\
\hline PR17 & Recherche et biosuivi & \\
\hline PR18 & Gestion des effets du changement climatique & \multirow{2}{*}{$\begin{array}{l}\text { Gestion des effets du } \\
\text { changement climatique } \\
\text { et des services } \\
\text { écosystémiques }\end{array}$} \\
\hline PR19 & Services écosystémiques & \\
\hline \multicolumn{3}{|c|}{ Résultats (Ouptuts) } \\
\hline R1 & \multicolumn{2}{|l|}{ Mise en œuvre du programme de travail } \\
\hline $\mathrm{R} 2$ & \multicolumn{2}{|l|}{ Résultats atteints } \\
\hline \multicolumn{3}{|c|}{ Effets/impacts (Outcomes) } \\
\hline E/l1 & \multicolumn{2}{|l|}{ Réalisation des objectifs de conservation } \\
\hline $\mathrm{E} / \mathrm{I} 2$ & \multicolumn{2}{|l|}{ État de conservation des valeurs désignées de l'aire protégée } \\
\hline $\mathrm{E} / \mathrm{I3}$ & \multicolumn{2}{|l|}{ Tendances de l'état de conservation des valeurs désignées de l'aire protégée } \\
\hline$E / 14$ & \multicolumn{2}{|l|}{ Effets/Impacts sur les communautés locales } \\
\hline$E / 15$ & \multicolumn{2}{|l|}{ Effets/Impacts sur l'atténuation et l'adaptation au changement climatique } \\
\hline$E / 16$ & \multicolumn{2}{|l|}{ Effets/Impacts sur les services écosystémiques } \\
\hline
\end{tabular}

\section{Poser les bases}

Dans plusieurs cas, le Formulaire est le premier exercice pour constituer une ligne de base (baseline) pour l'aire protégée. II constitue donc la Baseline T0 (temps zéro). Les informations seront obtenues à partir des ressources bibliographiques existantes, notamment le plan de gestion, des études, du suivi et les cas échant des connaissances de l'équipe de gestion de l'aire protégée. Pour les années suivantes, il sera uniquement question de mises à jour. II est donc normal que le premier remplissage du Formulaire prenne beaucoup plus de temps.

Les fiches suivantes concernent des éléments importants pour bien comprendre, remplir et analyser le Formulaire.

\begin{tabular}{|l|l|}
\hline FICHE 1 & Contexte de l'intervention et contexte de la gestion \\
\hline FICHE 2 & Services écosystémiques \\
\hline FICHE 3 & Menaces, pressions et soutiens extérieurs \\
\hline FICHE 4 & Cycle de gestion des aires protégées \\
\hline FICHE 5 & Évaluation de l'efficacité de la gestion \\
\hline
\end{tabular}




\section{FICHES ET EXERCICES POUR MAITRISER L'ÉVALUATION D’UNE AIRE PROTÉGÉE}

\section{FICHE 1 - Services écosystémiques}

(⿴) Les services écosystémiques sont les bienfaits que les hommes obtiennent des écosystèmes. Ces services sont nombreux, et peuvent être répartis en services d'approvisionnement tels que la nourriture et l'eau, services de régulation tels que la régulation des inondations et des maladies, services culturels tels que les bénéfices spirituels, récréatifs et culturels, et services de soutien qui maintiennent des conditions favorables à la vie sur Terre, tels que le cycle des éléments nutritifs.

Ce schéma illustre les liens entre les différentes catégories des services écosystémiques et les principales composantes du bien-être humain, et inclut des indications sur les liens possibles avec des facteurs socio-économiques.

Dans le Formulaire BIOPAMA, les services écosystémiques pris en compte correspondent à un service rendu ou à leur existence avec la possibilité de rendre un service à l'avenir. Par exemple, un aspect spirituel existant dans une aire protégée constitue une valeur ; sa protection et éventuellement sa valorisation représentent un service écosystémique rendu à prendre en compte dans la gestion de l'AP.

Voir fichier 5-8 WHO Ecosystem and human wb

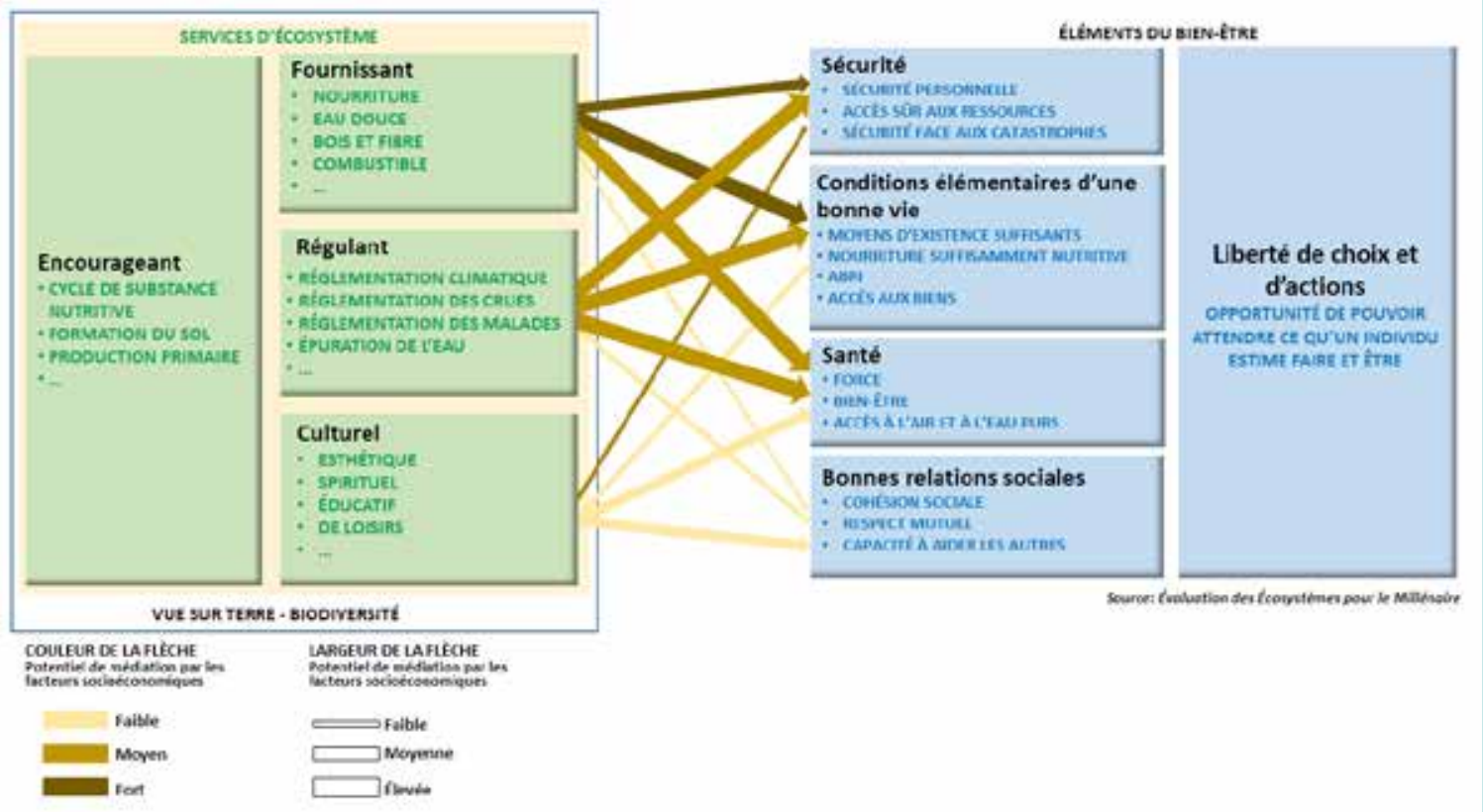

Schéma 1 - Liens entre les services fournis par les écosystèmes et le bien-être humain

Source : WHO (2005) Ecosystems and human well being 


\section{FICHES ET EXERCICES POUR MAITRISER L'ÉVALUATION D'UNE AIRE PROTÉGÉE}

\section{FICHE 2 - Menaces, pressions et soutiens extérieurs}

L'analyse du contexte de l'intervention, pour la définition des éléments importants dans la gestion de l'aire protégée, impose une connaissance détaillée des éléments exerçant une influence positive ou négative sur les activités de l'AP. À ce propos, le Formulaire propose deux instruments : le premier, plus sophistiqué, correspond à l'analyse des menaces ; le deuxième correspond à l'analyse des contraintes et des soutiens extérieurs.

\section{Menaces :}

(1) Dans le texte de référence en anglais, les pressions, les menaces et les vulnérabilités sont indiquées par le terme Threats - menaces. Bien que cette terminologie puisse être utilisée couramment, il est important d'un point de vue conceptuel de faire la distinction entre les trois aspects négatifs qui peuvent peser sur la gestion et la gouvernance des aires protégées :

La matrice d'estimation des menaces (Threat calculator ${ }^{6}$ ) proposée dans le Formulaire assure l'évaluation qualitative des trois forces et des aspects négatifs qui peuvent peser sur la gestion et la gouvernance d'une aire protégée.

6 Le concept de Threat calculator (Calculateur de menaces) a été développé par un groupe de travail mis en place par NatureServe en 2004 (http://www.natureserve.org/conservation-tools/conservation-rank-calculator). La classification des menaces a été adoptée par la CMAP de l'UICN, Salafsky et al. en 2008 (http://www.iucnredlist. org/technical-documents/classification-schemes/threats-classification-scheme).

\begin{tabular}{|l|l|}
\hline \multirow{2}{*}{ Pressions } & $\begin{array}{l}\text { Forces et éléments négatifs actuels qui ont un impact préjudiciable aujourd'hui sur } \\
\text { la conservation, la gestion et la valorisation durable des ressources naturelles de } \\
\text { l'écosystème de référence (AP, périphérie de l'AP, ou autre). }\end{array}$ \\
\hline Menaces & $\begin{array}{l}\text { Forces et éléments négatifs potentiels qui risquent d'avoir un impact préjudiciable } \\
\text { à l'avenir, à court ou long terme, mais qui n'existent pas encore aujourd'hui, sur la } \\
\text { conservation, la gestion et la valorisation durable des ressources naturelles dans } \\
\text { l'écosystème de référence (AP, périphérie de l'AP, ou autre). }\end{array}$ \\
\hline Vulnérabilités & $\begin{array}{l}\text { Faiblesses d'un système de conservation (p. ex. épizooties sporadiques, niveau élevé de } \\
\text { pauvreté, etc.) qui, s'ils se déclenchent, risquent d'avoir un impact préjudiciable sur le } \\
\text { patrimoine et les ressources naturelles à l'avenir, à court ou long terme. }\end{array}$ \\
\hline
\end{tabular}

\section{Exercice 2 - Identification des pressions, menaces et vulnérabilités}

L'exercice vise à réaliser une analyse détaillée des différents éléments de pression, menaces et vulnérabilités qui pèsent sur l'aire protégée afin d'établir un niveau de référence, d'assurer le suivi de l'évolution des menaces et d'effectuer une analyse des dispositions à prendre pour diminuer les effets négatifs qui pèsent sur la gestion et la gouvernance des aires protégées.

L'analyse du contexte de l'intervention du Parc national 2 au Pays 2 avait permis à l'équipe de gestion de mieux repérer l'importance croissante du conflit homme/éléphant et d'en tenir compte dans la gestion.

\section{Questions}

1. Par rapport au contexte de l'intervention et de gestion du parc, quels aspects pourraient indiquer l'importance croissante du conflit homme/éléphant ?

2. Par rapport au processus de gestion, quels éléments doivent prendre en compte des réponses adéquates au conflit homme/éléphant ? 


\section{FICHES ET EXERCICES POUR MAITRISER L'ÉVALUATION D'UNE AIRE PROTÉGÉE}

Exercice 3 - Structuration schématique des menaces
Dans l'aire protégée 3, Pays 3, un exercice sur les menaces a été conduit en adoptant comme outil une
séance de réflexion (brainstorming), suivie par une vérification des éléments de menace à l'aide de la
matrice présentée dans le Formulaire. L'analyse comparée a permis de remarquer, à l'aide du Calculateur
de menaces, l'existence de trois autres menaces, dont une particulièrement importante (surpâturage
dans les zones de gestion communautaire).
Questions
1. Considérez-vous la structuration systématique des éléments de la gestion et de la gouvernance
d'une aire protégée présentés dans le Formulaire comme un avantage ou un inconvénient ?
2. Considérez-vous que la structure très schématique des éléments présentés dans le Formulaire
puisse être supportée par d'autres instruments d'aide à la collecte des informations indispensables
à la gestion et la gouvernance des aires protégées ?
3. Préférez-vous introduire la demande de collecte des informations par d'autres outils pour lancer et
faciliter l'opération et, dans ce cas, que suggérez-vous comme outils de soutien ?

\section{Contraintes et supports extérieurs :}

L'analyse apporte des informations importantes sur le comportement et l'aptitude des acteurs en fonction aussi de leur pouvoir d'influence dans les différents processus de décision relatifs au contexte de l'intervention. Elle sert à mieux comprendre les interactions politicoinstitutionnelles, administratives et sociales des principaux acteurs avec l'aire protégée et leurs interventions de conservation, gestion et valorisation des ressources naturelles. L'analyse apporte aussi des éléments complémentaires sur le contexte de gouvernance et de gestion de l'aire protégée.

L'analyse est facilitée avec I'utilisation du graphique "Contrainte - Support - Pouvoir des acteurs ", proposé ci-dessous.

Le graphique permet de positionner dans un champ d'analyse idéal les différents acteurs intervenant dans le contexte de l'intervention par rapport à leur pouvoir d'influence négative (contrainte) ou positive (support/soutien) aux processus de gestion et de gouvernance entamés par l'aire protégée. Le graphique facilite l'identification et la valeur de l'influence des acteurs sur la gestion et la gouvernance de l'aire protégée. Les valeurs attribuées sont ainsi à reporter dans le point C2 pour le traitement, l'interprétation et la présentation statistique des données

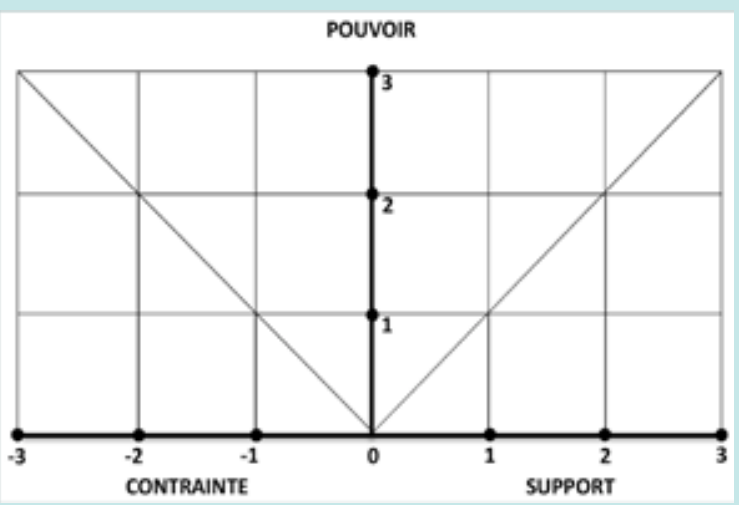

Schéma 2 - Graphique « Contrainte - Support - Pouvoir des acteurs » sur les interactions des principaux acteurs du contexte de l'intervention avec l'aire protégée

\section{Exercice 4 - Analyse Contrainte - Support -Pouvoir des acteurs}

Questions

1. Considérez-vous que l'analyse «Contrainte - Support - Pouvoir des acteurs » puisse aussi être utilisée pour mieux évaluer la typologie de gouvernance de l'aire protégée ?

2. Estimez-vous que l'appréciation précédente peut ou doit être renforcée par d'autres points du Formulaire et dans ce cas seriez-vous en mesure de les préciser?

3. Pouvez-vous suggérer les analyses nécessaires pour adopter des dispositions d'intervention ? 


\section{FICHES ET EXERCICES POUR MAITRISER L'ÉVALUATION D'UNE AIRE PROTÉGÉE}

\section{FICHE 3 - Contexte d'intervention et contexte de la gestion}

Le formulaire d'évaluation de l'efficacité de la gestion distingue le contexte de l'intervention (ou contexte élargi) et le contexte de la gestion (ou contexte spécifique de gestion de l'aire protégée).

\section{Contexte de l'intervention}

Le système de suivi et d'autoévaluation de la gestion des AP exige une parfaite connaissance du contexte de l'intervention dans lequel évolue la gestion de l'AP. Le terme " contexte d'intervention » est utilisé pour identifier l'ensemble des éléments directement et indirectement liés à la gestion et à la gouvernance des AP. II s'agit de l'environnement managérial de l'aire protégée. Le parc n'est pas une forteresse isolée, en dehors du système dans lequel il est situé. II comprend tous les facteurs externes à l'aire protégée et à son administration susceptibles d'influencer la gestion. Ces facteurs externes peuvent se situer au niveau local, national, régional et international, avec des degrés décroissants de contrôle du gestionnaire de l'aire protégée.
L'analyse, à l'aide des listes et des tableaux, soutient la validation et facilite l'identification des éléments à intégrer dans le cycle de gestion et plus spécifiquement le contexte de la gestion.

- Éléments considérés comme des aspects importants puisqu'ils peuvent orienter les décisions et les dispositions à prendre (p.ex. les statuts de classement, le contexte historique, socioéconomique, etc. ou les menaces ou vulnérabilités de l'aire protégée) ;

- Éléments à prioriser dans la gestion interne ou comme indicateurs de référence (p.ex. les espèces et les habitats clés, la gestion du personnel et des ressources diverses, les valeurs des conditions souhaitées pour évaluer l'effet et l'impact des efforts de conservation);

- Éléments au-delà des capacités d'intervention de l'aire protégée et à faire suivre par un niveau de décision supérieur puisqu'ils influencent positivement ou négativement la gestion (p.ex. des dispositions d'amélioration du statut de classement, mesures d'application des lois sur la conservation ou la gestion des ressources naturelles).

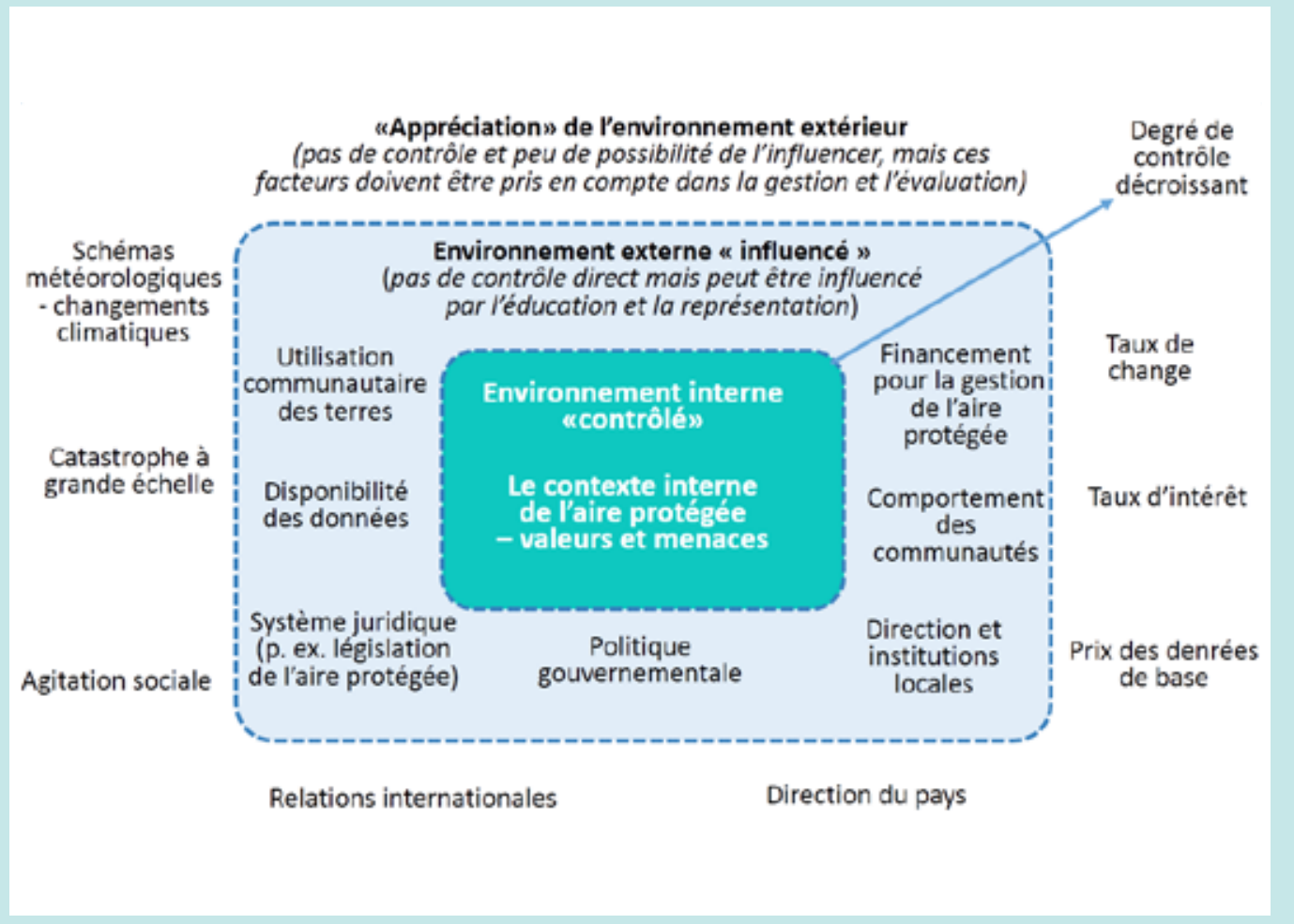

Schéma 3 - Environnement managérial d'une aire protégée (d'après Cusworth and Francks, 1993 in Hockings, et al.2008) 


\section{FICHES ET EXERCICES POUR MAITRISER L'ÉVALUATION D'UNE AIRE PROTÉGÉE}

\section{Contexte de la gestion}

(国) Le contexte de la gestion concerne l'environnement interne " contrôlé ». Les aires protégées sont créées pour des valeurs spéciales dont la connaissance est importante pour la planification et l'évaluation de la gestion. Le contexte de la gestion comprend la gouvernance/partenariat, Le schéma indicatif ci-dessous montre la connexion entre l'identification et la tendance des valeurs et des éléments importants relevés par le contexte d'intervention, et leur prise en compte dans le système d'évaluation de l'efficacité de gestion (voir Schéma 1). les valeurs et significations de I'AP, les menaces, les contraintes et soutiens extérieurs influençant directement la gestion quotidienne de l'AP.

\section{Exercice 5 - Valeurs : aspects importants}

II s'agit de disposer d'une manière synthétique et structurée des informations relatives aux statuts de classements, aux contextes (historiques, socioéconomique, etc.), aux menaces ou vulnérabilités de l'aire protégées, etc.

L'analyse du contexte de l'intervention du Parc national 1 du Pays 2 a permis à l'équipe de gestion de se rappeler du statut de Zone importante pour les oiseaux (GA 005) accordé en raison des critères A1, A2, $\mathrm{A} 3, \mathrm{~A} 4 \mathrm{i}, \mathrm{A} 4 \mathrm{iii}{ }^{*}$ et d'en tenir compte dans la gestion.

Questions

1. Comment expliquez-vous que l'équipe de gestion ne soit pas au courant de cette classification?

2. Quels changements cette classification pourrait-elle entraîner dans la gestion du PN 1 ?

3. Faut-il considérer la méconnaissance du statut de Zone importante pour les oiseaux comme une erreur relative au contexte de l'intervention ou de planification?

${ }^{*} A 1$. Espèces d'intérêt pour la conservation mondiale ; A2. Espèces à aire réduite ; A3. Espèces restreintes à un biome ; A4. Congrégations : i) Le site est connu ou dit abriter, régulièrement $\geq 1 \%$ d'une population biogéographique d'une espèce grégaire d'oiseau d'eau ; iii) Le site est connu ou dit abriter, régulièrement $\geq 20000$ d'oiseaux d'eau ou $\geq 10000$ paires d'oiseaux de mer d'une espèce ou plus ; Voir aussi la classification official http://www.birdlife.org/datazone/info/ibacritg/ob

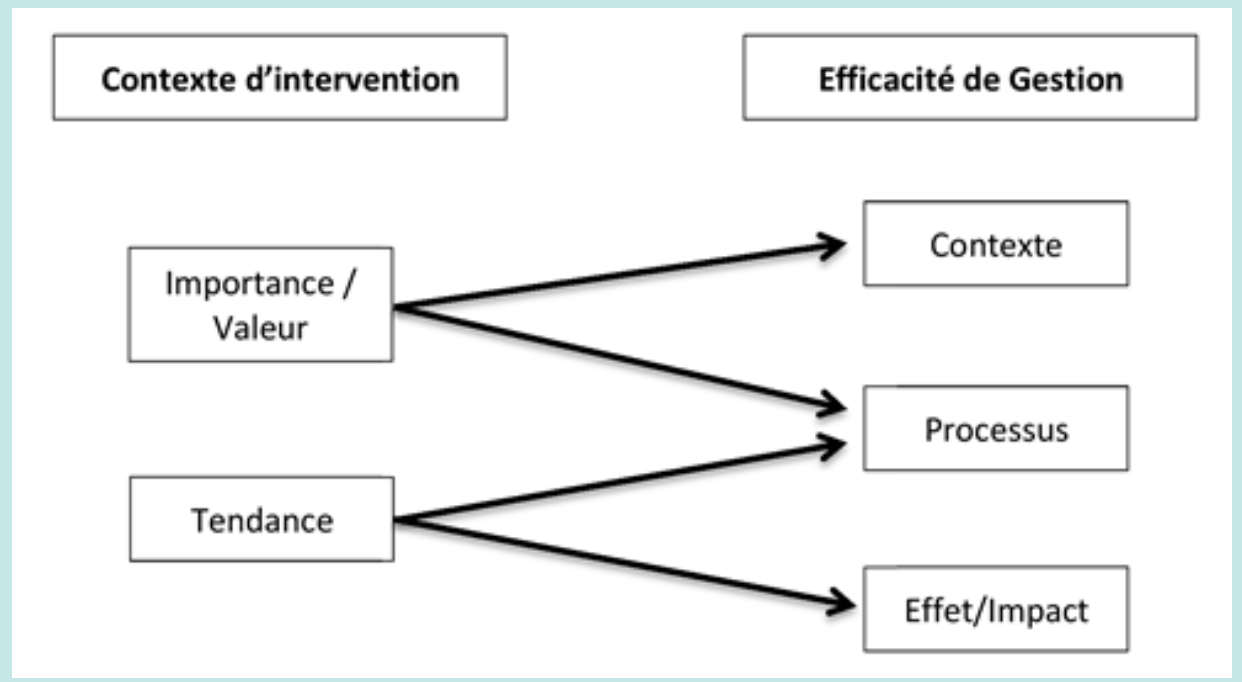

Schéma 4 - Liaison fonctionnelle entre les éléments du contexte d'intervention et l'efficacité de gestion 


\section{FICHES ET EXERCICES POUR MAITRISER L'ÉVALUATION D'UNE AIRE PROTÉGÉE}

\section{Éléments extérieurs au-delà des capacités d'intervention de l'aire protégée}

I| s'agit d'identifier les aspects importants pour solliciter les niveaux de décision supérieurs pour l'amélioration de la gestion de l'aire protégée (p.ex. des dispositions d'amélioration du statut de classement, des mesures d'application des lois sur la conservation ou sur la gestion des ressources naturelles, etc.)

\section{Exercice 6 - Influence sur la législation}

L'analyse du contexte de l'intervention de plusieurs aires protégées dans plusieurs pays a permis de définir les difficultés d'application des conventions internationales sur la conservation ou les conflits existants entre les dispositions liées à la conservation et l'utilisation des ressources naturelles par d'autres organismes étatiques. Il est donc nécessaire d'identifier et de faire avancer au niveau supérieur, d'une manière coordonnée entre les gestionnaires des aires protégées, les requêtes pour l'amélioration du contexte juridique d'application.

\section{Questions}

1. À votre avis, quelles sont les situations conflictuelles les plus dangereuses et menaçantes pour la bonne gestion des aires protégées ?

2. Dans quelle mesure l'aire protégée peut-elle influencer un changement dans les lignes de la politique nationale?

3. Quelles dispositions proposez-vous pour l'amélioration d'une ou de plusieurs situations de conflit dans la gestion des ressources naturelles ?

4. Avez-vous des exemples à partager avec vos collègues ?

\section{Exercice 7 - Changement du degré de classement}

L'analyse du contexte de l'intervention et de l'efficacité de la gestion du Parc national 2 au Pays 2 a permis de conclure que le décret de classement (aspect délimitation) du parc était à revoir. Les efforts de gestion avaient déjà permis d'atteindre une partie des objectifs du plan de gestion. Cependant, les valeurs à protéger et les menaces futures exigeaient une révision de la délimitation du parc pour assurer l'amélioration des processus de gestion de l'aire protégée.

\section{Questions}

1. Comment en est-on arrivé à définir que le décret de classement du parc était à revoir pour permettre une amélioration dans la gestion ?

- en discutant avec les responsables ;

- dans l'analyse croisée, entre quels éléments du cycle de gestion de l'aire protégée ?

- par l'analyse des menaces ;

- par une autre analyse (préciser, SVP) :. .

2. À votre avis et dans des cas identiques, n'est-il pas suffisant de revoir et de mettre à jour les documents de planification sans avoir recours à une demande pour le changement de classement ? 


\section{FICHES ET EXERCICES POUR MAITRISER L'ÉVALUATION D'UNE AIRE PROTÉGÉE}

\section{FICHE 4 - Cycle de gestion des AP}

Le cadre de la Commission mondiale des aires protégées (CMAP) se base sur l'idée selon laquelle la gestion efficace d'une aire protégée se caractérise par six étapes distinctes :

1. Cela commence par la compréhension du Contexte des valeurs et des menaces,

2. en passant par la Planification pour l'organisation et le plan des interventions visant des cibles bien définies,

3. l'allocation et la mise à disposition de ressources conséquentes (Intrants ou Inputs) et
4. la mise en œuvre des activités et des dispositions de gestion (Processus).

5. On aboutit à la production de biens et services ou (Extrants : Actions et Résultats ou Outputs),

6. qui entraînent des effets et les impacts nécessaires à atteindre les objectifs de conservation déterminés (Effets/Impacts ou Outcomes), le terme "Résultats » dans le diagramme doit être entendu dans le sens plus large et il est préférable d'utiliser le terme effet/impact correspondant au mot Outcome en anglais).

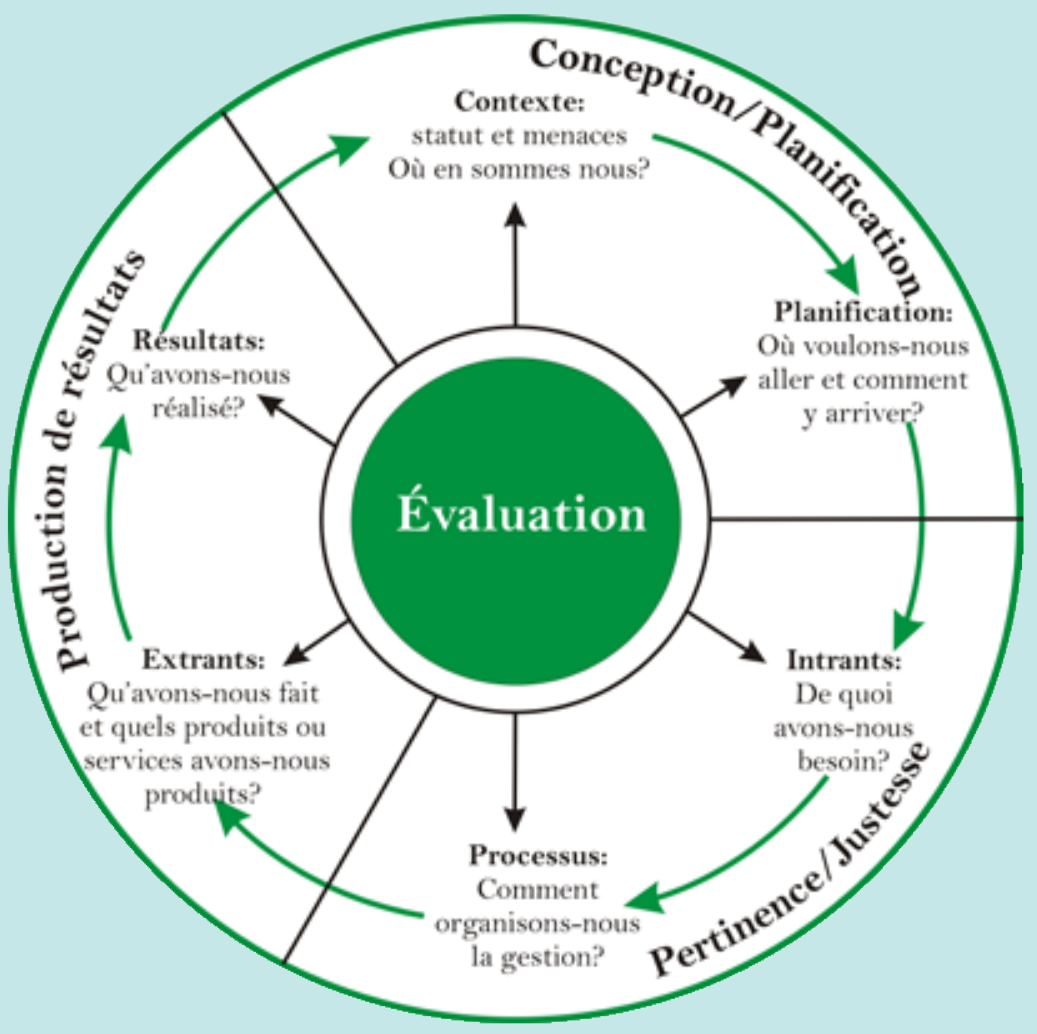

Schéma 5 - Cadre d'évaluation de l'efficacité de la gestion

\section{Exercice 8 - Pertinence de l'approche de gestion d'une AP}

\section{Questions}

1. Pensez-vous que la gestion et la gouvernance des aires protégées nécessitent cette complexité d'analyse et de mise en œuvre ?

2. Avez-vous des alternatives aux aspects conceptuels proposés (cycle de gestion, processus tourné vers la réalisation des effets et impacts pour atteindre des objectifs) ?

3. Considérez-vous que les approches proposées puissent être utilisées aussi pour déterminer des objectifs de gouvernance, d'amélioration des conditions de vie des populations riveraines, du maintien et de la viabilité des services écosystémiques, etc. et gérer les interventions établis à ce but ? 


\section{FICHES ET EXERCICES POUR MAITRISER L'ÉVALUATION D’UNE AIRE PROTÉGÉE}

\section{Encadré 12 - Les éléments à mesurer}

Le cadre de la Commission mondiale des aires protégées vise à fournir une méthodologie globale dans le développement du processus d'évaluation. Le cadre est fondé sur l'idée qu'une bonne gestion d'une aire protégée peut être appréhendée à partir de six entrées distinctes :

\section{Contexte : où en est-on ?}

Cette question vise à examiner la conservation et d'autres valeurs d'une aire protégée, son état actuel, les menaces auxquelles elle est exposée et les possibilités qu'elle offre, y compris le contexte politique dans son ensemble. Lorsqu'on effectue une évaluation afin de dresser une liste des priorités de gestion au sein d'un réseau d'aires protégées, ou pour déterminer le temps et les ressources à consacrer à une aire déterminée, il peut s'agir de la principale tâche à accomplir.

\section{Planification : à quoi veut-on aboutir?}

Cette question touche les résultats que l'on compte obtenir dans l'aire protégée. Une évaluation doit examiner la pertinence de la législation et des politiques nationales concernant les aires protégées. Elle peut permettre d'examiner la conception d'une aire protégée en fonction de l'intégrité et de l'état de cette ressource.

\section{Intrants : de quoi a-t-on besoin ?}

Cette question touche à la suffisance des ressources par rapport aux objectifs de gestion d'un réseau ou d'un site, en se fondant principalement sur une estimation du personnel, des fonds, du matériel et des installations nécessaires, au sein de l'organisme responsable ou sur le terrain, tout en tenant compte de l'importance des partenariats.

\section{Processus : comment peut-on s'y prendre ?}

Cette question permet de juger de la pertinence des systèmes et des processus de gestion par rapport aux objectifs de gestion d'un réseau ou d'un site. L'évaluation doit tenir compte d'une variété d'indicateurs comme les enjeux que représentent l'entretien courant, la pertinence des approches concernant les collectivités locales, et les différents types de gestion des ressources naturelles et culturelles.

5. Résultats (Outputs) : qu'a-t-on accompli et quels produits ou services a-t-on générés ?

Ces questions concernent l'évaluation des extrants et ce qui a été accompli grâce à la gestion. Elles visent à examiner le degré de réalisation des objectifs et d'exécution des programmes ou des plans de travail. On peut habituellement fixer ces objectifs dans le cadre de plans de gestion ou de programmes annuels de travail. Le contrôle des extrants consiste bien plus à déterminer si les mesures ont permis d'atteindre les objectifs visés (évaluation des résultats) qu'à apprécier si les activités ont été accomplies comme prévues et si la mise en œuvre des plans de gestion à long terme donne lieu à des progrès.

\section{Effets / Impacts (Outcomes) : quels résultats a-t-on obtenus ?}

Cette question sert à établir si la gestion a été fructueuse compte tenu des objectifs d'un plan de gestion, des plans nationaux et des buts correspondants à la catégorie d'aires protégées établie par l'UICN. L'évaluation des résultats est plus éloquente lorsque la législation ou les politiques nationales, de même que les plans de gestion de sites particuliers, sont assortis d'objectifs concrets. Les approches visant l'évaluation des résultats impliquent la surveillance à long terme de l'état des ressources biologiques et culturelles d'un site, des aspects socio-économiques de son utilisation et des incidences de sa gestion sur les collectivités locales. En dernière analyse, l'évaluation des résultats est une mesure tangible de l'efficacité de la gestion.

Source avec adaptation : Triplet, P. (2009). Manuel de gestion des aires protégées d'Afrique francophone. Awely, Paris, p. 1215. 


\section{FICHES ET EXERCICES POUR MAITRISER L'ÉVALUATION D'UNE AIRE PROTÉGÉE}

\section{Exercice 9 - Objectifs et planification dans la gestion d'une AP}

L'exercice vise à présenter des exemples d'application (ou non) des principes du cycle de gestion et du système de Planification - Suivi - Évaluation dans une aire protégée. L'approfondissement de ces principes conceptuels permet d'établir une bonne introduction à la compréhension de la logique d'élaboration et de l'utilisation du Formulaire ainsi que des opportunités d'analyses pour l'amélioration de la gestion et de la gouvernance des aires protégées.

L'utilisation du Formulaire dans une aire protégée 3, Pays 1, avait relevé l'absence d'objectifs dans le plan de gestion en cours d'élaboration, mais à un niveau d'avancement important.

\section{Questions}

1. Pensez-vous que ce type d'omission aurait pu se produire si le personnel de gestion et d'élaboration du plan de gestion avait suffisamment intériorisé les aspects conceptuels du processus [cycle de gestion] orienté à l'acquisition des effets et impacts pour atteindre des objectifs ?

2. Pensez-vous que cette découverte aurait pu se faire aussi facilement en l'absence de l'utilisation du Formulaire ou bien son utilisation systématique assure en même temps une analyse du contexte de l'intervention, de la planification et l'évaluation des dispositions et des actions de conservation ?

3. Pensez-vous que le Formulaire puisse vraiment constituer une base pour l'analyse des documents de planification de la gestion et de la gouvernance des aires protégées ?

4. Pensez-vous que le Formulaire puisse aussi être utilisé pour fournir les éléments de base permettant de constituer provisoirement une base de planification des interventions orientées sur des objectifs en l'absence d'un plan de gestion ou dans le cas de son expiration ? 


\section{FICHES ET EXERCICES POUR MAITRISER L'ÉVALUATION D'UNE AIRE PROTÉGÉE}

\section{FICHE 5 - Évaluation de l'efficacité de la gestion}

(1) L'efficacité de la gestion donne la mesure dans laquelle les produits d'une intervention ont eu des effets/impacts directs, et que ces derniers ont contribué à l'objectif recherché (objectif de la gestion). L'efficacité des interventions de conservation peutêtre synthétiquement analysée à l'aide du cadre d'évaluation de l'efficacité de la gestion des aires protégées de la commission mondiale des aires protégées (CMAP) de l'UICN (Hockings et al, 2008) pour les aires protégées et les réseaux d'aires protégées.

Tableau 2 - Résumé du cadre de la CMAP de l'UICN
Le cadre de la CMAP de I'UICN a pour objectif d'établir des directives pour l'élaboration de systèmes d'évaluation et encourager la mise en place de normes d'évaluation et de compte-rendu. C'est un outil de référence, pour les gestionnaires et autres personnes concernées, qui contribue à l'harmonisation des processus d'évaluation dans le monde. En conséquence, le Formulaire prend en compte l'application du cadre de la CMAP de I'UICN ou les outils ou la méthodologie qui se basent sur ce schéma. Le tableau 1 présente un résumé du cadre et le tableau 2 illustre synthétiquement les objectifs et les critères d'évaluation du cadre de la CMAP de I'UICN.

\begin{tabular}{|c|c|c|c|}
\hline $\begin{array}{l}\text { Éléments } \\
\text { d'évaluation }\end{array}$ & Explication & Critères évalués & $\begin{array}{l}\text { Point focal de } \\
\text { l'évaluation }\end{array}$ \\
\hline $\begin{array}{l}\text { Contexte de } \\
\text { la gestion }\end{array}$ & $\begin{array}{l}\text { La situation actuelle } \\
\text { Évaluation de l'importance, des } \\
\text { menaces et du cadre politique }\end{array}$ & $\begin{array}{l}\text { - Importance } \\
\text { - Menaces } \\
\text { - Vulnérabilité } \\
\text { - Contexte national } \\
\text { - Partenaires }\end{array}$ & Situation \\
\hline Planification & $\begin{array}{l}\text { La situation recherchée } \\
\text { Évaluation de la planification de } \\
\text { l'aire protégée }\end{array}$ & $\begin{array}{l}\text { - Contexte juridique et politique } \\
\text { de l'aire protégée } \\
\text { - Définition des priorités, des } \\
\text { cibles d'intervention et des } \\
\text { objectifs de l'aire protégée par } \\
\text { rapport aux objectifs du réseau } \\
\text { d'aires protégées nationale } \\
\text { - Plan de gestion et plan de travail } \\
\text { annuel ou pluriannuel }\end{array}$ & Faisabilité \\
\hline Intrants & $\begin{array}{l}\text { Les besoins } \\
\text { Évaluation des ressources } \\
\text { nécessaires }\end{array}$ & $\begin{array}{l}\text { - Ressources humaines } \\
\text { - Ressources financières } \\
\text { - Moyens disponibles }\end{array}$ & Ressources \\
\hline Processus & $\begin{array}{l}\text { La gestion } \\
\text { Évaluation du mode de gestion }\end{array}$ & $\begin{array}{l}\text { - Adéquation et pertinence des } \\
\text { processus de gestion }\end{array}$ & $\begin{array}{l}\text { Faisabilité et } \\
\text { Efficience }\end{array}$ \\
\hline $\begin{array}{l}\text { Actions/ } \\
\text { Résultats }\end{array}$ & $\begin{array}{l}\text { Les actions et les résultats de la } \\
\text { gestion } \\
\text { Évaluation de la mise en œuvre } \\
\text { des programmes et activités de } \\
\text { gestion ; produits et services fournis }\end{array}$ & $\begin{array}{l}\text { - Résultats des activités de } \\
\text { gestion } \\
\text { - Services et produits }\end{array}$ & $\begin{array}{l}\text { Efficience et } \\
\text { Efficacité }\end{array}$ \\
\hline Effet/Impact & $\begin{array}{l}\text { Les effets et les impacts de la } \\
\text { gestion } \\
\text { Évaluation des effets et des impacts } \\
\text { et de leur contribution aux objectifs }\end{array}$ & $\begin{array}{l}\text { Effets et impacts : atteints par } \\
\text { les dispositions et les activités } \\
\text { de gestion des objectifs de } \\
\text { gestion et de conservation }\end{array}$ & $\begin{array}{l}\text { Efficacité et } \\
\text { faisabilité }\end{array}$ \\
\hline
\end{tabular}




\section{FICHES ET EXERCICES POUR MAITRISER L'ÉVALUATION D'UNE AIRE PROTÉGÉE}

Tableau 3 - Objectifs et critères d'évaluation du cadre de la CMAP de l'UICN

\begin{tabular}{|c|c|c|c|c|c|c|}
\hline \multirow{2}{*}{$\begin{array}{l}\text { Éléments } \\
\text { du cycle } \\
\text { de gestion }\end{array}$} & \multicolumn{2}{|c|}{ Conception } & \multicolumn{2}{|c|}{ Conformité/Adéquation } & \multicolumn{2}{|c|}{ Effets } \\
\hline & $\begin{array}{l}\text { Contexte de la } \\
\text { gestion }\end{array}$ & Planification & Intrants & Processus & $\begin{array}{l}\text { Actions/ } \\
\text { résultats }\end{array}$ & $\begin{array}{l}\text { Effets/ } \\
\text { impacts }\end{array}$ \\
\hline $\begin{array}{l}\text { Objet de } \\
\text { l'évaluation }\end{array}$ & $\begin{array}{l}\text {-Évaluation de } \\
\text { l'importance, } \\
\text { des pressions, } \\
\text { des menaces, } \\
\text { des politiques } \\
\text { environnementales, } \\
\text { etc. }\end{array}$ & $\begin{array}{l}\text {-Évaluation de } \\
\text { la conception } \\
\text { et de la } \\
\text { planification } \\
\text { de l'aire } \\
\text { protégée }\end{array}$ & $\begin{array}{l}\text { •Évaluation } \\
\text { des } \\
\text { ressources } \\
\text { nécessaires } \\
\text { pour mener } \\
\text { à bien la } \\
\text { gestion }\end{array}$ & $\begin{array}{l}\text { - Évaluation } \\
\text { de la } \\
\text { manière dont } \\
\text { la gestion } \\
\text { est effectuée }\end{array}$ & $\begin{array}{l}\text {-Évaluation } \\
\text { de la } \\
\text { mise en } \\
\text { œuvre des } \\
\text { programmes } \\
\text { et des } \\
\text { actions de } \\
\text { gestion } \\
\text { - Livraison des } \\
\text { produits et } \\
\text { des services }\end{array}$ & $\begin{array}{l}\text { •Évaluation } \\
\text { des effets } \\
\text { et des } \\
\text { impacts; et } \\
\text { dans quelle } \\
\text { mesure les } \\
\text { objectifs } \\
\text { sont atteints }\end{array}$ \\
\hline $\begin{array}{l}\text { Critères } \\
\text { évalués }\end{array}$ & $\begin{array}{l}\text { - Importance/Valeurs } \\
\text { Pressions } \\
\text { - Menaces } \\
\text { - Vulnérabilité } \\
\text { - Intervenants } \\
\text { - Contexte national }\end{array}$ & $\begin{array}{l}\text { - Politique et } \\
\text { législation } \\
\text { sur les aires } \\
\text { protégées } \\
\text { - Conception } \\
\text { du système } \\
\text { des AP } \\
\text { - Conception } \\
\text { de l'AP } \\
\text { spécifique } \\
\text { - Planification } \\
\text { de la gestion }\end{array}$ & $\begin{array}{l}\text { - Ressources } \\
\text { disponibles } \\
\text { pour l'aire } \\
\text { protégée }\end{array}$ & $\begin{array}{l}\text { - Adéquation } \\
\text { des } \\
\text { processus } \\
\text { de gestion } \\
\text { et évaluation } \\
\text { dans } \\
\text { laquelle les } \\
\text { processus } \\
\text { établis ou } \\
\text { acceptés } \\
\text { sont mis en } \\
\text { œuvre }\end{array}$ & $\begin{array}{l}\text { - Résultats } \\
\text { des } \\
\text { dispositions } \\
\text { de gestion } \\
\text { - Services et } \\
\text { produits }\end{array}$ & $\begin{array}{l}\text { - Effets et } \\
\text { impacts : } \\
\text { réalisation } \\
\text { des objectifs } \\
\text { de gestion }\end{array}$ \\
\hline
\end{tabular}

Puisque la terminologie peut générer des incompréhensions, il est important de faire la distinction entre les aspects fondamentaux qui

Un exemple est donné dans le tableau suivant. déterminent l'appréciation de la gestion et la gouvernance des aires protégées, à savoir :

\begin{tabular}{|l|l|}
\hline Action & $\begin{array}{l}\text { L'action décrit l'accomplissement de quelque chose et cela contribue à générer des } \\
\text { résultats. }\end{array}$ \\
\hline Résultat & $\begin{array}{l}\text { Le résultat est ce que l'on obtient en agissant dans un but défini à travers des actions et } \\
\text { cela contribue à générer un effet. }\end{array}$ \\
\hline Effet & $\begin{array}{l}\text { L'effet décrit comment et dans quelle mesure le résultat ou les résultats ont contribué à un } \\
\text { changement et cela contribue à générer un impact. }\end{array}$ \\
\hline Impact & $\begin{array}{l}\text { L'impact désigne les conséquences (indirectes ou non) sur l'état de la conservation et cela } \\
\text { contribue à atteindre l'objectif (objectif de conservation). }\end{array}$ \\
\hline Efficacité \\
de la
\end{tabular}$\quad \begin{aligned} & \text { L'efficacité de la gestion permet de savoir dans quelle mesure les produits d'un processus } \\
& \text { ont eu des effets/impacts directs et les effets/impacts directs ont contribué à l'objectif } \\
& \text { (objectif de gestion). }\end{aligned}$




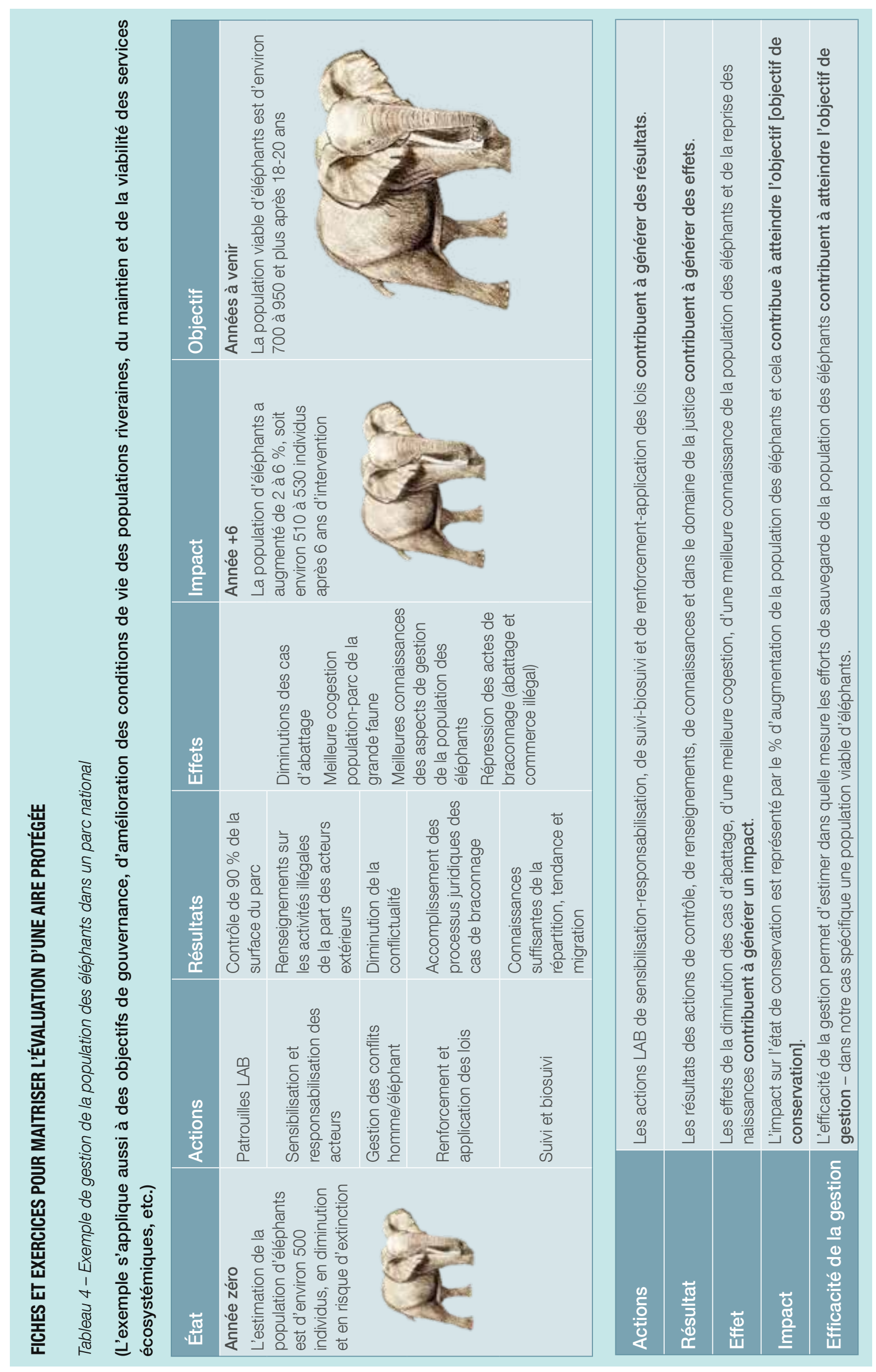


Attention : Lors des discussions, il est fréquent de généraliser la situation avec celle des autres parcs ou même du pays. II faut donc faire attention de bien rester dans le cadre de l'aire protégée en question.

\section{CE QU'IL FAUT RETENIR DU} FORMULAIRE

- Le Formulaire est une liste de contrôle : i donne toutes les options possibles, mais certaines peuvent ne pas concerner l'AP. ॥ faut passer en revue chaque rubrique une par une, ne pas partir du principe que certaines ne sont pas nécessaires pour l'AP.

- Le Formulaire n'a pas un but scientifique, mais un objectif d'évaluation de gestion. Le coach doit faire confiance à l'appréciation et à l'intuition du Conservateur et de son équipe. Toutefois, le coach doit se baser sur les documents existants, notamment le plan de gestion et d'aménagement.

- Le Formulaire a également un rôle d'alerte (clignotant ou avertisseur) pour l'équipe de gestion concernant des aspects oubliés ou négligés et dont certains paramètres méritent d'être suivis à l'avenir.

- C'est un travail itératif. Les différentes rubriques permettent de regarder sous différents angles. II ne faut pas avoir peur de faire un va-et-vient entre les différents éléments du Formulaire.

- Lorsque cela ne concerne pas votre cas : ne rien mettre ou suivre les indications de remplissage (dans certains cas la formule statistique impose de mettre la valeur conventionnelle de -99 qui ne sera pas objet de calcul). Zéro est déjà une valeur.

- Utiliser la colonne «Observations » pour les compléments d'informations.

- Après la mission sur le terrain, le Conservateur et son équipe peuvent revenir sur le Formulaire pour approfondir et préciser certains aspects ; certainement pas pour changer des valeurs en vue d'améliorer l'estimation de l'évaluation.

- Le Formulaire concerne uniquement le parc (et non la zone adjacente ni la zone tampon), même si ces zones ont un impact sur le parc.

\subsubsection{Avant d'exploiter le Formulaire}

Avant de commencer, demander aux participants si la démarche et les éléments de base sont clairs. II pourra être important de rappeler :

- Le système de barèmes des réponses 0/1/2/3 qui permet de déterminer avec les valeurs « 0 et 1 » des appréciations négatives et « 2 et 3 » des estimations positives. Dans le cas d'analyse des tendances vous devez utiliser les valeurs -3 / -2 / -1 pour des évolutions négatives ; 0 pour des situations stables ; +1/ +2 / +3 pour les évolutions positives. Pour établir l'appréciation d'un sujet, il faut commencer par déterminer si l'estimation est négative ou positive. Dans le premier cas, déterminer si l'estimation est très négative (0) ou juste négative (1). Dans le deuxième cas, déterminer si l'estimation est positive (2) ou très positive (3). Le coach n'est pas le décideur, mais il oriente le Conservateur vers la réponse.

- La méthodologie étape par étape : Certaines rubriques peuvent ne pas concerner I'AP mais il faut les lire une par une, sans en sauter certaines. De même, s'il y a un problème identifié (ou ayant un point négatif) à résoudre, il est préférable de terminer l'analyse du sujet de conservation et ensuite de fixer la/les problématiques à analyser en vue de formuler les solutions à adopter (voire les rubriques : «Détermination des objectifs-cibles ».

Conditions pour bien remplir le Formulaire :

- Participation des personnes concernées ;

- Certaines cases peuvent ne pas être remplies si les informations ne sont pas disponibles, (mais si le sujet est important au point de vue de la gestion/gouvernance, il faudra prendre en compte la démarche pour disposer des données) ;

- Logistique bien établie : lieu calme de réunion, vidéoprojecteur...

- Prévoir un temps suffisant : au moins deux jours de travail pour un premier remplissage.

\subsubsection{Pré-remplissage (module Contexte de l'intervention)}

Le pré-remplissage doit être réalisé bien avant l'atelier de remplissage et de la manière la plus approfondie possible. Le coach est chargé de faire 
le pré-remplissage, ce qui lui permettra de bien• connaître l'aire protégée. II remplira principalement la partie sur le contexte de l'intervention. II pourra ensuite passer en revue avec le Conservateur ces rubriques de manière plus rapide pour la validation ou le complètement des informations manquantes.

Les sources pour faire le pré-remplissage sont :

- Le plan de gestion ;

- Le plan financier ;

- Le plan de travail annuel ;

- Les autres documents de planification et d'organisation de la gestion de l'aire protégée au niveau local et national ;

- Les résultats des études, des recherches, du suivi ;
Les connaissances et l'information grise de l'équipe de gestion de l'aire protégée ;

- Les rapports déjà établis par l'institution nationale: rapport d'activités, rapport de recherches ;

- Les données de l'Observatoire numérique pour les aires protégées (DOPA) et de l'Observatoire des forêts d'Afrique centrale (OFAC) ;

La base de données mondiale de l'UICN sur les aires protégées http://www. protectedplanet.net/

Le pré-remplissage insuffisant des éléments du contexte de l'intervention entraînera une augmentation du temps d'échanges avec les équipes des parcs.

\section{Liste des codes : CONTEXTE DE L'INTERVENTION}

CTX .1. Informations générales sur l'aire protégée

CTX.1.0 Responsable de la compilation du fichier

CTX.1.1 Données de base

CTX.1.2 Gouvernance et partenariat

CTX.1.3 Statut spécial : Patrimoine mondial, Programme Homme et biosphère, Site Ramsar, Zones importantes pour les oiseaux, etc.

CTX.1.4 Localisation géographique

CTX 1.5 Appartenance à un réseau

CTX.1.6 Vision - mission - objectifs

CTX.1.7 Références des contextes historique, politique, juridique et institutionnel

CTX.1.8 Autres éléments spécifiques de l'aire protégée à disposition pour mieux identifier le caractère de protection

CTX.1.9 Détermination des objectifs-cibles de la conservation et des indicateurs relatifs à la classification, gouvernance, partenariat, etc. de l'AP

CTX.2. Superficies, limites et indice de forme, niveau de contrôle de l'aire protégée

CTX.2.1 Superficie de l'AP et du contexte de conservation (à valider par les responsables de I'AP)

CTX.2.2 Zonage/secteurs de l'aire protégée

CTX.2.3 Niveau de contrôle de l'aire protégée

CTX.2.4 Contexte territorial de référence de l'aire protégée

CTX.2.5 Détermination des objectifs-cibles de la conservation et des indicateurs relatifs aux superficies, limites et indice de forme, niveau de contrôle de l'aire protégée

CTX.3. Ressources humaines, financières et matérielles de l'aire protégée

CTX.3.1 Taille et composition du personnel de l'aire protégée 
CTX.3.2 Ressources financières

CTX.3.2.1 Budget et coûts de gestion

CTX.3.2.2 Budget disponible

CTX 3.2.3 Lignes budgétaires du plan opérationnel/plan de travail budgétisé annuellement

CTX 3.2.4 Financement des partenaires

CTX.3.3 Ressources matérielles (infrastructures, équipement, installations)

CTX. 3.4 Détermination des objectifs-cibles de la conservation et des indicateurs relatifs aux ressources humaines et financières / partenariat et en moyens dans la gestion de l'aire protégée

CTX.4. Éléments clés des aires protégées terrestres et marines : i) espèces phares, menacées, endémiques ; ii) habitats ; iii) valeurs culturelles de l'aire protégée et iv) valeurs touristiques

CTX.4.1 Espèces animales phares, menacées, endémiques, importantes, invasives, exploitées, avec données insuffisantes (mammifères, oiseaux, amphibiens, reptiles, poissons) choisies comme indicateurs de l'aire protégée

CTX.4.2 Espèces végétales phares, menacées, endémiques, importantes, invasives, exploitées, avec données insuffisantes, choisies comme indicateurs de l'aire protégée

CTX.4.3 Écosystèmes et habitats

CTX.4.3.1 Écosystèmes et habitats à caractéristiques importantes et significatives de l'aire protégée

CTX.4.3.2 Présence, étendue et répartition des habitats et stratus marins clés

CTX.4.4 Maintien de la couverture des sols (land cover) ou type de terrain physique - forêt, eau, routes, etc.

CTX.4.5 (Pour les aires protégées à gestion des ressources naturelles] Utilisation légale mais non durable de l'aire protégée terrestre ou marine (utilisation des terres land use ou des ressources par l'homme)

CTX. 4.6 Détermination des objectifs-cibles de conservation et des indicateurs relatifs aux éléments clés et aux espèces phares, menacées, endémiques et avec données insuffisantes de l'AP

CTX.5. Pressions et menaces sur l'aire protégée

CTX.5.1 Pressions et menaces qui pèsent sur l'aire protégée

CTX.5.2 Détermination des objectifs-cibles de conservation et des indicateurs relatifs aux pressions et menaces qui pèsent sur l'AP

CTX.6. Changement climatique et conservation

CTX.6.1 Changement climatique et conservation - Éléments importants

CTX.6.2 Changement climatique et conservation - Tendances des valeurs

CTX.6.3 Détermination des objectifs-cibles de conservation et des indicateurs relatifs au changement climatique et à la conservation

CTX.7 Services écosystémiques et interdépendance des collectivités de l'aire protégée envers ces services

CTX.7.1 Services écosystémiques et dépendance des collectivités de l'aire protégée envers ces services Éléments importants

CTX.7.2 Services écosystémiques et dépendance des collectivités de l'aire protégée envers ces services Tendances des valeurs

CTX.7.3 Détermination des objectifs-cibles au maintien des services écosystémiques et à la dépendance des collectivités de l'aire protégée envers ces services - Tendances des valeurs 


\section{CONTEXTE DE L'INTERVENTION}

Cette partie constitue une sorte de monographie de l'aire protégée.

\section{Informations générales sur l'aire protégée}

\section{CTX.1.0}

\section{Responsables de la compilation du fichier}

Les questions 1.0 permettent d'identifier les référents et les responsables ayant compilé le Formulaire ou fourni les informations, d'enregistrer la date de fin du remplissage du Formulaire et la durée de l'exercice.

Voir le lien avec CTX1.2. Si la gouvernance est centralisée, il serait normal que le remplissage soit uniquement réalisé par des agents de l'État. Dans le cas d'une AP au mode de gouvernance partagée, cela amènerait à poser des questions.

\section{CTX.1.1}

\section{Données de base}

Les questions 1.1 permettent d'identifier et de rapporter les données essentielles de l'aire protégée ainsi que son appartenance à des unités écologiques d'échelle supérieure (biome et écorégion). Les informations permettent aussi d'apporter les corrections nécessaires des banques de données au niveau national, régional et global.

\section{CTX.1.2}

\section{Gouvernance et partenariat}

Les questions CTX 1.2 visent:

- à préciser la typologie de la gouvernance selon la classification de référence fournie par I'UICN et, éventuellement, d'en souligner les spécificités ;

- à indiquer les partenariats en cours en soutien de l'action de gestion et de gouvernance de l'aire protégée.

Pour information : le terme "gouvernance » dans le Formulaire doit être entendu comme le processus de prise de décision dans la gestion des aires protégées. Les partenariats ne concernent que les aspects de collaboration dans les activités de gestion, un aspect très important dans les interventions de conservation.

\section{CTX.1.3}

Statut spécial : Patrimoine mondial, Homme et biosphère (MAB), Site Ramsar, Zones importantes pour les oiseaux, etc.
Les éléments CTX 1.3 permettent de préciser les classifications et statuts ainsi que les critères de désignation de l'aire protégée. Certains éléments demandés sont pré-renseignés sur la base des données disponibles au niveau des institutions internationales concernées, mais ces informations doivent être vérifiées et modifiées en cas d'erreur par les gestionnaires de l'aire protégée.

Il faudra faire dans le pré-remplissage une vérification sur les sources internationales. II peut arriver que tous les responsables de l'équipe de gestion ne connaissent pas tous les statuts qui concernent leur AP.

\section{CTX.1.4}

\section{Localisation géographique}

Les éléments CTX 1.4 permettent de préciser la localisation géographique et la localisation administrative de la zone protégée (province, région, etc.).

\section{CTX.1.5}

\section{Appartenance à un réseau}

Les éléments CTX 1.5 permettent de préciser l'appartenance technique/administrative de l'aire protégée à un réseau de conservation. Outre l'éventuelle appartenance à des réseaux officiels (par ex. parcs transfrontaliers) ou à un paysage (p.ex. du Programme régional de l'Afrique centrale pour l'environnement (CARPE)), il est important de citer ici l'appartenance de l'aire protégée à des réseaux de conservation spéciaux au niveau national et international. Lorsque l'aire protégée appartient à un réseau ou système de conservation, les autres aires protégées associées doivent être citées également.

\section{CTX.1.6}

\section{Vision, mission, objectifs}

Les éléments CTX 1.6 permettent de faire ressortir la vision, la mission et le/les objectif(s) de l'aire protégée et qui sont normalement définis dans le plan de gestion. Plus spécifiquement, le/les objectif(s) de l'aire

Si la vision, la mission ou les objectifs ne sont pas clairement définis ou s'ils sont dépassés, faire le lien avec CTX 1.9. Ce n'est donc pas le moment de définir la vision dans cette section, mais de noter en CTX 1.9 qu'il faudra prendre en considération des changements dans les efforts de conservation. Il est néanmoins possible de rédiger des premières hypothèses concernant la révision de la vision, de la mission ou du/des objectif(s) de l'aire protégée et qui seront intégrés dans les instruments de gestion. 
protégée peuvent aussi être définis au niveau local (par• ex. maintien des services écosystémiques au profit des populations riveraines), au niveau national (par ex. protection d'une espèce, d'habitats particuliers ou de valeurs culturelles), ou au niveau international (par ex. protection d'une espèce endémique, contribution au maintien d'un patrimoine partagé).

Une autre façon de définir la vision : Comment voyez-vous le parc à l'avenir, d'ici 10-20 ans ?

\section{CTX.1.7}

\section{Références des contextes historique, socioéconomique, politique, juridique et institutionnel}

Les éléments CTX 1.6 permettent de faire ressortir les aspects les plus importants des contextes historique, socioéconomique, politique (au niveau du pays), juridique et institutionnel, qui ont (ou ont eu) une influence importante, négative ou positive, sur le contexte de l'intervention actuelle, et en conséquence ont influencé ou influencent les interventions de la conservation dans l'aire protégée. II est important de renseigner les aspects négatifs (points faibles) et positifs (points forts) assortis d'éventuelles observations concernant l'influence des éléments historiques, sociaux et socioéconomiques, politiques (au niveau du pays), juridiques et institutionnels, etc.

\section{CTX.1.8}

\section{Autres éléments spécifiques de l'aire protégée à disposition pour mieux identifier le caractère de protection}

Les éléments CTX 1.7 permettent aux gestionnaires de l'aire protégée de mentionner des aspects et de fournir des informations qui n'auraient pas été abordées dans les points précédents, mais qui sont importantes pour mieux identifier les spécificités et caractériser la gestion adoptée ou à adopter pour l'aire protégée.

\section{CTX.1.9}

\section{Détermination des objectifs-cibles de conservation et des indicateurs relatifs aux aspects de : gouvernance et partenariat, statut, réseau d'appartenance, mission, contexte historique, socioéconomique, politique, juridique et institutionnel, autres que l'aire protégée}

À la fin du chapitre " Informations générales sur l'aire protégée » du Formulaire, un tableau permet d'identifier les éléments importants à prendre en compte dans la gestion de l'aire protégée. Dans le cas, le tableau est organisé pour insérer : les lignes de base de l'état de conservation ;

- les conditions souhaitées ou à atteindre (objectif) ;

- $\quad$ et les valeurs de référence (benchmarks) à utiliser pour l'amélioration de la gestion.

S'il y a eu un vide ou un problème identifié dans CTX 1 à 8, il faut le mentionner ici (surtout dans les cas où le plan de gestion est obsolète ou absent).

Les valeurs définies peuvent être utilisés pour la gestion et le suivi des activités de l'aire protégée et plus spécifiquement pour les phases de planification, recherche des ressources (intrants), processus, détermination des résultats et des objectifs d'impact.

\section{Superficies, limites et indice de forme,} niveau de contrôle de l'aire protégée

\section{CTX. 2.1}

\section{Superficies de l'aire protégée et du contexte de conservation (à valider par les responsables de l'aire protégée)}

Les éléments CTX 2.1 permettent de fournir une série d'éléments sur la surface et la forme de l'aire protégée. Plus spécifiquement, les valeurs de la surface sont analysées en fonction de la typologie et des réseaux d'appartenance de l'aire protégée. Les informations doivent permettre de relever les différences - si elles existent - entre les valeurs de la surface protégée rapportée par les différentes sources d'information. Les différences dans la détermination de la surface du domaine classé sont dues à des erreurs de transmission, aux estimations historiques par rapport à celles d'aujourd'hui plus précises, etc. Ce point doit également permettre de déterminer la relation entre la surface de l'aire protégée et le contexte plus général de conservation au niveau national et transfrontalier. Enfin, la note doit permettre le transfert d'informations relatives à l'existence de limites géo-référenciées (donc certaines et univoques) et de données SIG officielles.

\section{CTX. 2.2}

\section{Zonage/secteurs de I'AP}

L'élément CTX 2.2 permet de rapporter les informations essentielles sur le zonage et les aspects principaux des différents secteurs de gestion et de conservation de l'aire protégée. Les informations relatives au zonage pourront être utilisées dans le cadre du contexte de l'intervention ainsi que l'évaluation de l'efficacité de la gestion. 


\section{CTX. 2.3}

\section{Niveau de contrôle de l'aire protégée}

Les éléments CTX 2.3 permettent une réflexion concernant le niveau de contrôle actuel de l'aire protégée. À ce propos, plusieurs méthodes de mesure de la surface contrôlée sont proposées : hectares, km², hommes/jours de patrouille, \% de la surface ou autres si existantes. Le Formulaire permet aussi d'estimer le niveau de contrôle par secteur si cette démarche peut aider à la détermination de la valeur totale ou plus utile sur le plan de l'organisation de la gestion de l'aire protégée. Pour mieux préciser le niveau de contrôle, qui peut varier en fonction des spécificités du parc, le Formulaire introduit des informations concernant la surface soumise au suivi écologique et les valeurs de l'utilisation légale/illégale de l'aire protégée (utilisation des terres par l'homme dans le cas des aires protégées à gestion des ressources naturelles). Les valeurs connues et disponibles sont introduites au préalable pour permettre aux responsables de l'aire protégée de les vérifier.

\section{CTX. 2.4}

\section{Contexte territorial de référence de l'aire protégée}

Les éléments CTX 2.5 permettent de déterminer le contexte territorial dans lequel agit et interagit l'aire protégée. La détermination et la connaissance du contexte territorial d'intervention servent à mieux connaître les relations en termes de gouvernance et d'approche intersectorielle. De même, la connaissance du contexte territorial de référence permet de mieux estimer les menaces et les pressions qui pèsent sur l'aire protégée afin de mieux évaluer et examiner les aspects liés à l'efficacité de la gestion. Les valeurs connues et disponibles sont introduites au préalable pour faciliter l'analyse et permettre aux responsables de l'aire protégée de les vérifier.

\section{CTX. 2.5}

\section{Détermination des objectifs-cibles de conservation et des indicateurs relatifs aux superficies, limites et indice de forme, niveau de contrôle de l'aire protégée}

À la fin du chapitre "Superficies, limites et indice de forme, niveau de contrôle de l'aire protégée " du Formulaire, un tableau permet d'identifier les éléments importants à prendre en compte dans la gestion de l'aire protégée. Dans ce cas, le tableau est organisé pour insérer :

- les lignes de base de l'état de conservation ;

- les conditions souhaitées ou à atteindre (objectif) ;

- et les valeurs de référence (benchmarks) à utiliser pour l'amélioration de la gestion.
Les valeurs définies peuvent être utilisées pour la gestion et le suivi des activités de l'aire protégée et plus spécifiquement pour les phases de planification, recherche des ressources (intrants), processus, détermination des résultats et des objectifs d'impact.

\section{Ressources humaines, financières et matérielles de l'aire protégée}

\section{CTX. 3.1}

\section{Taille et composition du personnel de l'aire protégée}

Les éléments CTX 3.1 permettent d'identifier les ressources humaines employées directement dans la gestion de l'aire protégée. La liste des effectifs doit comprendre le personnel étatique ainsi que toutes les autres ressources humaines employées dans les différentes activités du parc par les partenaires de l'aire protégée. L'organisation et la classification des postes du personnel du parc sont normalement tirées des documents de planification de l'aire protégée et doivent être paramétrées pour correspondre aux dispositions d'organisation et d'encadrement du personnel par l'administration publique.

À la question : "Y a-t-il un effectif idéal ? » la réponse varie suivant plusieurs critères : la forme de l'aire, les menaces, les enjeux (statut/ menaces), le financement...

\section{CTX. 3.2}

\section{Ressources financières}

\section{CTX. 3.2.1}

Budget et coûts de gestion

Les éléments CTX 3.2.1 permettent de reporter les estimations financières à partir du Plan de gestion/ Plan financier et les estimations des coûts de fonctionnement à partir du Plan opérationnel/Plan de travail annuel. Les valeurs sont rapportées en fonction de la surface pour déterminer le coût au $\mathrm{km}^{2}$ par an.

\section{CTX. 3.2.2}

\section{Budget disponible}

Les éléments CTX 3.2.2 analysent les ressources mises réellement à la disposition de la gestion par les différents intervenants et les éventuelles recettes issues de la valorisation de la conservation de la biodiversité. La ligne prévoit la division en budget de fonctionnement et d'investissement.

\section{CTX. 3.2 .3}

Lignes budgétaires du plan opérationnel/plan de travail budgétisé annuellement 
Les éléments CTX 3.2.3 permettent de connaitre les lignes budgétisées et de pouvoir faire une comparaison entre priorités d'intervention et les ressources financières mises à la disposition, entre interventions, d'évaluer l'absorption du budget des différentes lignes, etc.

\section{CTX. 3.2.4}

\section{Financements des partenaires}

Les éléments CTX 3.2.4 permettent d'associer en termes d'engagement (temps et montant) les ressources mises à la disposition de la gestion par les différents intervenants de conservation de la biodiversité.

Face à la réticence à communiquer les ressources financières, ou parfois même l'incapacité à montrer de manière transparente le niveau de gestion, remplir ce qui est possible. Noter les actions à faire pour améliorer la situation financière et budgétaire dans les "Objectifs".

\section{CTX. 3.3}

\section{Ressources matérielles (infrastructures, équipement, etc.) - Disponibilité des infrastructures, des équipements et des installations}

Les éléments CTX 3.3, par un tableau long et très détaillé, permettent d'analyser la disponibilité des infrastructures, équipement, moyens mis à la disposition de la gestion de l'aire protégée. Les gestionnaires de l'AP peuvent utiliser le tableau comme inventaire des plus importantes ressources matérielles et pour identifier l'existence et programmer les nécessités.

CTX. 3.4

\section{Détermination des objectifs-cibles de conservation et des indicateurs relatifs aux ressources humaines et financières/appui des partenariats et en moyens dans la gestion de l'aire protégée}

À la fin du chapitre "Ressources humaines, financières et matérielles de l'aire protégée " du Formulaire, un tableau permet d'identifier les éléments importants à prendre en compte dans la gestion de l'aire protégée. Dans ce cas, le tableau est organisé pour insérer :

- les lignes de base de l'état de conservation ;

- les conditions souhaitées ou à atteindre (objectif) ;

- et les valeurs de référence (benchmarks) à utiliser pour l'amélioration de la gestion.
Les valeurs définies peuvent être utilisées pour la gestion et le suivi des activités de l'aire protégée et plus spécifiquement pour les phases de planification, recherche des ressources (intrants), processus, détermination des résultats et des objectifs d'impact.

\section{Éléments clés (AIRE PROTÉGÉE TERRESTRE/MARITIME) : i) espèces phares, menacées, endémiques, importantes, invasives, exploitées, mais avec données insuffisantes ; ii) habitats ; iii) changements dans la couverture des sols; et iv) gestion des ressources naturelles}

Pour cette partie, la Liste rouge des espèces menacées et la Liste CITES des espèces protégées peuvent aider à déterminer les valeurs biologiques de l'aire protégée.

Voir www.iucnredlist.org \& www.cites.org

Les paramètres relatifs aux éléments clés sont à utiliser pour les aires protégées terrestres, marines et mixtes. Pour les habitats marins, il existe un tableau spécifique (CTX 4.3.2).

\section{CTX. 4.1}

\section{Espèces animales phares, menacées, endémiques, importantes, invasives, exploitées, avec données insuffisantes (mammifères, oiseaux, amphibiens, reptiles, poissons) choisies comme indicateurs de l'aire protégée}

Les éléments CTX 4.1 cherchent à la fois à mieux cibler l'intervention de la conservation et à mieux organiser les informations relatives aux animaux clés et emblématiques de l'aire protégée. La gestion de l'aire protégée peut obtenir des résultats plus importants s'il est possible d'identifier et de cibler l'intervention de la conservation au profit des animaux symboliques et importants. Le personnel de l'aire protégée doit préciser dans le tableau les espèces les plus importantes, à savoir les espèces phares, menacées, endémiques, invasives, exploitées, avec données insuffisantes, etc. II est possible aussi d'identifier les animaux emblématiques selon une caractérisation différente comme les espèces charismatiques, parapluies, à conflit et architecte (voir ci-dessous) :

- Espèce "phare » ou charismatique : utilisée pour générer de l'intérêt et un appui financier pour la conservation de toutes les espèces sauvages qui partagent ses habitats ; 
- Espèce «parapluie ", dont la protection garantit la sécurité pour la biodiversité en général et pour l'industrie du tourisme ;

- Espèce « à conflit » homme-faune et qui constitue une problématique aigüe, à minimiser pour réduire la conflictualité entre les parties dans le même contexte de l'intervention ;

- Espèce " architecte ", capable de modifier les habitats à l'avantage ou au détriment d'espèces végétales et animales à l'échelle locale ou plus large, selon la nature et l'étendue de l'impact.

Après l'identification des espèces animales les plus importantes, le tableau propose d'introduire les estimations des populations par rapport à un état de conservation de base ou niveau de base (baseline), l'état (de conservation) souhaité à l'avenir, l'actuelle tendance et la fiabilité de l'information. Dans certains cas, ces informations sont difficilement disponibles et caractérisées par un faible niveau de fiabilité, toutefois leur analyse est importante pour l'évaluation de l'efficacité de la gestion de l'aire protégée.

\section{CTX. 4.2}

\section{Espèces végétales phares, menacées, endémiques, importantes, mais avec données insuffisantes, invasives, exploitées - choisies comme indicateurs de l'aire protégée}

La détermination de ces paramètres CTX. 4.2, relatif aux espèces végétales, peut suivre les indications du point précédent CTX. 4.1, même si les définitions des espèces parapluies, à conflit et architectes ne sont généralement pas utilisées pour les espèces végétales.

\section{CTX. 4.3}

\section{Écosystèmes et habitats}

\section{CTX. 4.3.1}

Écosystèmes et habitats à caractéristiques importantes et significatives de l'aire protégée

Les paramètres CTX. 4.3.1, relatif aux écosystèmes et aux habitats, peuvent suivre les indications des points précédents CTX. 4.1 et 4.2. Les gestionnaires, sur la base des paramètres relatifs à la spécificité, à l'endémisme ou à leur caractère unique ou irremplaçable ${ }^{7}$, doivent déterminer les habitats terrestres et d'eau douce les plus importants de l'aire protégée. L'identification des habitats emblématiques ou mis en danger servira pour mieux cibler l'intervention de la conservation.

7 Élément de grande valeur, rare ou unique, qui ne peut pas être remplacé.
CTX. 4.3.2

Présence, étendue et répartition des habitats et stratus marins clés

Les paramètres CTX. 4.3.2 permettent de déterminer la présence, l'étendue et la répartition des habitats marins clés. Les informations sur les habitats doivent permettre l'organisation et l'évaluation de la gestion de l'aire protégée marine.

\section{CTX. 4.4}

Maintien de la couverture des sols (ou type de terrain physique - forêt, eau, routes, etc.) [valeurs totales voir point 2.2]

Les paramètres CTX. 4.4 visent à déterminer l'état et l'évolution de la couverture des sols selon le Système de classification de la couverture des sols (Land Cover Classification System - LCCS). Cet élément du Formulaire contient la liste et la surface ou pourcentage des catégories identifiées pour l'aire protégée spécifique issue de l'information provenant des institutions internationales. Les valeurs et les tendances affichées doivent contribuer à la détermination des valeurs de gestion de l'aire protégée.

\section{CTX. 4.5}

\section{[Pour les aires protégées à gestion des} ressources naturelles] Utilisation légale, mais non durable de l'aire protégée terrestre ou marine (utilisation de la terre - land use - ou des ressources par l'homme) [valeurs totales voir point 2.2]

Les paramètres CTX. 4.5 doivent s'appliquer seulement aux aires protégées qui, par leur typologie de classement (par ex. catégorie VI de I'UICN : Aire protégée avec utilisation durable des ressources naturelles) ou par la présence des droits coutumiers, permettent l'utilisation de la terre et des ressources naturelles à l'intérieur du domaine classé.

\section{CTX. 4.6}

Détermination des objectifs-cibles de conservation et des indicateurs relatifs aux éléments clés et aux espèces phares, menacées, endémiques ou avec des données insuffisantes de l'aire protégée

À la fin du chapitre "Éléments clés (aire protégée terrestre/maritime) : i) espèces phares, menacées, endémiques, importantes, invasives, exploitées, mais avec données insuffisantes ; ii) habitats ; iii) changement dans la couverture des sols et valeurs culturelles de l'aire protégée et iv) gestion des ressources naturelles » du Formulaire, un tableau permet d'identifier des éléments importants à 
prendre en compte dans la gestion de l'aire protégée. Dans ce cas, le tableau est organisé pour insérer :

- les lignes de base de l'état de conservation ;

- les conditions souhaitées ou à atteindre (objectif) ;

- $\quad$ et les valeurs de référence (benchmarks) à utiliser pour l'amélioration de la gestion.

Les valeurs définies peuvent être utilisées pour la gestion et le suivi des activités de l'aire protégée et plus spécifiquement pour les phases de planification, recherche des ressources (intrants), processus, détermination des résultats et des objectifs d'impact.

\section{Pressions et menaces sur l'aire protégée}

\section{CTX. 5.1}

\section{Pressions et menaces qui pèsent sur l'aire protégée}

Les paramètres CTX 5.1 sont composés d'un long tableau d'analyse des pressions, menaces et vulnérabilités de l'aire protégée. Ce tableau est dénommé Calculateur des menaces (du modèle Threat calculator $^{8}$, adapté pour le système d'analyse et d'évaluation du Formulaire). Le but est de formuler une analyse détaillée des menaces (ce terme sera utilisé pour représenter les pressions, menaces et vulnérabilités qui pèsent sur l'aire protégée). Dans ce tableau, il faut noter chaque pression pertinente pour l'aire protégée en fonction des informations concernant : a) l'impact ; b) l'ampleur ; c) la durée ; d) l'évolution au cours d'une période donnée (préciser le nombre d'années) ; et e) la probabilité d'une menace à l'avenir. Les résultats de cette analyse seront utilisés pour améliorer le processus de gestion visant à annuler ou à réduire les impacts des menaces sur la biodiversité. L'analyse des menaces qui pèsent sur l'aire protégée est facilitée

Se référer à la fiche $n^{\circ} 2$ si nécessaire.

par la visualisation des valeurs par un histogramme.

\section{CTX. 5.2}

\section{Détermination des objectifs-cibles de conservation et des indicateurs relatifs aux pressions et aux menaces qui pèsent sur l'aire protégée}

À la fin du chapitre "Pressions et menaces sur l'aire protégée » du Formulaire, un tableau permet

\footnotetext{
8 Le calculateur a été développé par un groupe de travail mis en place par NatureServe en 2004 (http://www.natureserve.org/conservationtools/conservation-rank-calculator). La classification des menaces a été adoptée par la CMAP de l'UICN, Salafsky et al. en 2008 (http:// www.iucnredlist.org/technical-documents/classification-schemes/ threats-classification-scheme).
}

d'identifier les éléments importants à prendre en compte dans la gestion de l'aire protégée. Dans ce cas, le tableau est organisé pour insérer :

- les lignes de base de l'état de conservation ;

- les conditions souhaitées ou à atteindre (objectif) ;

- $\quad$ et les valeurs de référence (benchmarks) à utiliser pour l'amélioration de la gestion.

Les valeurs définies peuvent être utilisées pour la gestion et le suivi des activités de l'aire protégée et plus spécifiquement pour les phases de planification, recherche des ressources (intrants), processus, détermination des résultats et des objectifs d'impact.

\section{Changement climatique et conservation}

\section{CTX. 6.1}

\section{Changement climatique et conservation - Éléments importants}

Les paramètres CTX 6.1 permettent d'identifier les éléments importants du changement climatique liés à l'aire protégée. II s'agit d'analyser les éléments de valeur et de ressources naturelles importants de l'aire protégée affectée, actuellement ou potentiellement à l'avenir, par le changement climatique, à savoir : classification, importance et mission du domaine de conservation, espèces clés, habitats, services écosystémiques, etc. L'analyse doit également prendre en compte les analyses spécifiques, les études pertinentes, les interventions en cours (par ex. la REDD+).

\section{CTX. 6.2}

\section{Changement climatique et conservation - Tendances des valeurs}

Les paramètres CTX 6.2 permettent de relever les principaux effets du changement climatique, par exemple l'augmentation des menaces, les variations dans la présence et la répartition des espèces et des habitats, etc., et les éventuelles actions d'atténuation ou d'adaptation proposées ou adoptées. Ces éléments de base permettront d'analyser les éventuelles actions d'atténuation ou d'adaptation au changement climatique mises en œuvre ou à adopter.

\section{CTX. 6.3}

\section{Détermination des objectifs-cibles de conservation et des indicateurs relatifs au changement climatique et conservation}

À la fin du chapitre "Changement climatique et conservation » du Formulaire, un tableau permet d'identifier des éléments importants à prendre en compte dans la gestion de l'aire protégée. Dans ce cas, le tableau est organisé pour insérer : 
- les lignes de base de l'état de conservation ;

- les conditions souhaitées ou à atteindre (objectif) ;

- et les valeurs de référence (benchmarks) à utiliser pour l'amélioration de la gestion.

Les valeurs définies peuvent être utilisées pour la gestion et le suivi des activités de l'aire protégée et plus spécifiquement pour les phases de planification, recherche des ressources (intrants), processus, détermination des résultats et des objectifs d'impact.

\section{Services écosystémiques et dépendance des collectivités de l'aire protégée envers ces services}

CTX. 7.1

\section{Services écosystémiques et dépendance des collectivités de l'aire protégée envers ces services - Éléments importants}

Le paramètre CTX. 7.1 permet de guider les gestionnaires de l'aire protégée dans l'identification des services écosystémiques rendus par l'aire protégée au bénéfice du bien-être humain. Les paramètres CTX 7.1 permettent de classer les services écosystémiques identifiés en trois grandes catégories spécifiques à l'aire protégée : approvisionnement ; régulation ; et culturel. Les services écosystémiques pris en compte par le questionnaire correspondent à un service rendu et à leur existence avec la potentialité de rendre un service à l'avenir. Par exemple, un aspect spirituel existant dans une aire protégée constitue une valeur; sa protection et éventuellement sa valorisation représentent un service écosystémique rendu à protéger.

Pour les services écosystémiques relatifs à la catégorie "approvisionnement ", il est important de noter que l'attribution du caractère légal ou illégal de l'approvisionnement dépend de la catégorie de classification de l'aire protégée et des éventuelles utilisations coutumières tolérées à l'intérieur du domaine classé. La catégorie " culturel » inclut les services culturels (qui comprennent aussi les valeurs touristiques), spirituels et religieux. La catégorie culturelle doit prend en compte les valeurs existantes historiquement et culturellement à l'intérieur de l'aire protégée.

Les paramètres CTX 7.1 proposent de relever les services écosystémiques existants, valorisés ou potentiels de l'aire protégée. Le Formulaire facilite l'analyse par la présentation d'un tableau avec les services écosystémiques rendus pour le bien-être humain commun dans les aires protégées, mais les évaluateurs peuvent insérer d'autres éléments que ceux proposés.

L'analyse doit permettre de promouvoir l'intégration de ces valeurs dans les systèmes de gestion de l'aire protégée visant à assurer le maintien et la valorisation des services écosystémiques.

\section{CTX. 7.2}

\section{Pressions et menaces qui pèsent sur l'aire protégée}

Les paramètres CTX 7.2 doivent permettre de relever les tendances des principaux services écosystémiques identifiés dans le point précédent (CTX. 7.1). Ces éléments de base permettront d'analyser les éventuelles mesures de gestion à adopter pour la préservation et la valorisation des services écosystémiques.

\section{CTX. 7.3}

\section{Détermination des objectifs liés au maintien des services écosystémiques et à la dépendance des collectivités de l'aire protégée envers ces services - Tendances des valeurs}

À la fin du chapitre "Services écosystémiques et dépendances des collectivités de l'aire protégée envers ces services " du Formulaire, un tableau permet d'identifier les éléments importants à prendre en compte dans la gestion de l'aire protégée. Le tableau est organisé pour insérer :

- les lignes de base de l'état de conservation ;

- les conditions souhaitées ou à atteindre (objectif) ;

- et les valeurs de référence (benchmarks) à utiliser pour l'amélioration de la gestion.

Les valeurs définies peuvent être utilisées comme éléments importants pour la gestion et le suivi des activités de l'aire protégée et plus spécifiquement pour les phases de planification, recherche des ressources (intrants), processus, détermination des résultats et des objectifs d'impact.

\subsubsection{Utilisation du Formulaire}

Cette deuxième étape concerne la révision, la fin du remplissage du contexte de l'intervention et l'exploitation du module du contexte de la gestion. Le remplissage du Formulaire se fait au cours d'un atelier de travail avec les responsables de la gestion de l'aire protégée. Normalement, il s'agit du Conservateur 
et son équipe de gestion. Les populations et les partenaires locaux peuvent y participer, selon la typologie de gouvernance de l'aire protégée.

Conditions souhaitées pour la séance de remplissage :

- Les coaches ont exploité les informations et les données disponibles sur l'aire protégée ;

- Le pré-remplissage a été réalisé ;

- Le binôme de coach a déjà effectué une visite du site pour mieux comprendre les réalités ;
- L'équipe de gestion du parc a été préalablement informée et est disposée à consacrer 2 à 3 jours de travail pour cette séance, et par conséquent, un endroit favorable au travail (calme, climatisé, si nécessaire) a été identifié.

Programme général du déroulement de la séance de remplissage :
JOUR 1

- Rappel général : objectifs de la mission, étapes franchies, Formulaire, méthodologie de remplissage, échelles

- Contexte de l'intervention : vérification des données et ajouts des informations manquantes

- Contexte de la gestion et planification
JOUR 2

- Contexte de la gestion et planification (suite)

- Intrants

- Processus

- Résultats
JOUR 3

- Effets et impacts

- Analyse des résultats, des effets et de l'impact des efforts de conservation

- Formulation des propositions d'amélioration de la gestion

- Prochaines étapes de l'accompagnement

\section{Encadré 13 - Enseignements tirés à la suite du remplissage du Formulaire lors des phases test \\ Le Formulaire en aide à la planification, le suivi et l'évaluation de l'efficacité de la gestion du BIOPAMA présente une certaine difficulté pour sa compréhension et demande du temps pour son déroulement en raison des multiples aspects pris en compte dans l'analyse du contexte de l'intervention et de l'efficacité de la gestion. Cependant, les difficultés initiales diminuent à mesure que l'exercice apporte les premiers supports en termes de connaissance du contexte et des réponses en aide à la gestion.}

L'expérience acquise au cours des tests montre deux cas de figure :

Dans les aires protégées disposant de moins de capacités et, en conséquence, d'informations d'aide à la décision, la méfiance envers l'outil est forte. L'équipe du parc considère l'outil comme un système d'évaluation extérieur plutôt que comme un support interne à l'amélioration de la gestion. La méfiance envers l'outil diminue par étapes et notamment après la découverte que le Formulaire : i) est un instrument destiné à l'auto-évaluation, qui doit donc être paramétré spécifiquement pour l'aire protégée ; ii) fournit un apport pour la planification ; iii) permet la prise de décision sur des aspects concrets de la gestion ; et iv) introduit l'harmonisation du système de suivi par rapport aux interventions de conservation.

Dans les aires protégées disposant de davantage de capacités de gestion et en conséquence d'informations d'aide à la décision, l'outil a été estimé utile voire très utile, car il permet : i) une amélioration de la compréhension du contexte de l'intervention ; ii) la simplification et la focalisation des instruments de planification vers la gestion ; iii) un soutien pour une meilleure priorisation des interventions ; iv) une possible amélioration de l'organisation du travail ; v) le perfectionnement du système de suivi, etc.

En conclusion, malgré diverses appréciations du Formulaire BIOPAMA de la part des responsables des aires protégées, les avis sont globalement positifs.

RAPAC/ECOFAC (2014) Analyse de l'état de la conservation et la de gestion des aires protégées d'Afrique centrale, p. 36 


\section{Liste des codes: EVALUATION DE L'EFFICACITE DE LA GESTION}

\begin{tabular}{|l|l|}
\hline C1.Contexte de la gestion \\
\hline C1 & Valeur et importance \\
\hline C1.1 & Valeur et Importance : Sous-indicateur Gouvernance / Partenariat \\
\hline C1.2 & Valeur et Importance : Sous-indicateur : Classifications \\
\hline C1.3 & $\begin{array}{l}\text { Valeur et Importance : Sous-indicateur : Espèces animales et végétales phares, menacées, } \\
\text { endémiques, exploitées, invasives, avec données insuffisantes }\end{array}$ \\
\hline C1.4 & $\begin{array}{l}\text { Valeur et Importance: Sous-indicateur : Habitats terrestres et marins et couverture des sols/ } \\
\text { changement dans l'utilisation des sols }\end{array}$ \\
\hline C1.5 & Valeur et importance : Sous-indicateur : Changement climatique \\
\hline C1.6 & Valeur et importance : Sous-indicateur : Services écosystémiques \\
\hline C2 & Contraintes ou appuis externes \\
\hline C3 & Menaces \\
\hline & $\begin{array}{l}\text { Détermination des objectifs-cibles de conservation et des indicateurs relatifs au contexte de la } \\
\text { gestion de l'aire protégée }\end{array}$ \\
\hline
\end{tabular}

\section{Planification}

P1 Adéquation des dispositions législatives et réglementaires

P2 Conception et configuration de l'aire protégée

P3 Démarcation de l'aire protégée

P4 Plan de gestion

P5 Plan de travail

P6 $\quad$ Objectifs de l'aire protégée

Détermination des objectifs-cibles de conservation et des indicateurs aux exercices et outil de planification de l'aire protégée

Intrants

I1 Informations de base

12 Personnel

I3 Budget actuel

$14 \quad$ Sécurisation du budget

15 Infrastructures, équipements et installations

Détermination des objectifs-cibles de conservation et des indicateurs relatifs aux intrants

nécessaires pour la mise en œuvre de la planification de l'aire protégée

\begin{tabular}{|l|l|}
\hline 1 & Systèmes et processus de gestion interne \\
\hline PR1 & Capacités et formation du personnel \\
\hline PR2 & Politiques et procédures de gestion des ressources humaines \\
\hline PR3 & Systèmes et processus de gestion des ressources humaines \\
\hline PR4 & Administration et leadership interne \\
\hline PR5 & Gestion comptable et financière \\
\hline PR6 & Entretien des infrastructures, des équipements et des installations \\
\hline 2 & Protection et gestion \\
\hline PR7 & Gestion des valeurs et des aspects importants de l'aire protégée \\
\hline PR8 & Systèmes de protection des valeurs et des aspects importants de l'aire protégée \\
\hline PR9 & Contrôle de l'aire protégée \\
\hline PR10 & Application des lois \\
\hline
\end{tabular}




\begin{tabular}{|l|l|}
\hline 3 & Protection et gestion \\
\hline PR11 & Implication des communautés locales, des ayants droit et des parties prenantes \\
\hline PR12 & Avantages/ Assistance approprié au profit des collectivités \\
\hline PR13 & Relations avec les acteurs \\
\hline 4 & Gestion du tourisme \\
\hline PR14 & Gestion des visiteurs \\
\hline PR15 & Visiteurs et impacts \\
\hline 5 & Suivi et recherche \\
\hline PR16 & Systèmes de suivi des ressources naturelles et culturelles \\
\hline PR17 & Recherche et biosuivi \\
\hline 6 & Gestion des effets du changement climatique et des services écosystémiques \\
\hline PR18 & Gestion des effets du changement climatique \\
\hline PR19 & Services écosystémiques \\
\hline & $\begin{array}{l}\text { Détermination des objectifs-cibles de conservation et des indicateurs relatifs au Processus de } \\
\text { l'aire protégée }\end{array}$ \\
\hline Résultats (Ouptuts) \\
\hline R1 & Mise en œuvre des principales activités du programme de travail annuel ou pluriannuel \\
\hline R2 & Réalisation des principaux résultats attendus du plan de travail annuel ou pluriannuel \\
\hline Effets et impacts (Outcomes) \\
\hline E/11 & Réalisation des objectifs de conservation \\
\hline E/12 & État de conservation des valeurs désignées de l'aire protégée \\
\hline E/13 & Tendances de l'état de conservation des valeurs désignées de l'aire protégée \\
\hline E/14 & Effets et impacts sur les communautés locales \\
\hline E/15 & Effets et impacts sur l'atténuation et l'adaptation au changement climatique \\
\hline E/16 & Effets et impacts sur les services écosystémiques \\
\hline
\end{tabular}

\section{CONTEXTE DE LA GESTION}

Très important de bien préciser que nous sommes maintenant dans le contexte de la gestion

\section{C1}

\section{Valeur et importance}

C1.1

Valeur et Importance : Sous-indicateur

Gouvernance/Partenariat

L'élément C1.1 permet d'estimer la valeur et l'importance des formes de gouvernance et de partenariats adoptées ou qu'il serait nécessaire d'adopter pour améliorer l'efficacité de la gestion de l'aire protégée. L'analyse est supportée par la suggestion des éventuelles typologies de collaboration et d'intégration entre les parties prenantes. II est possible de compléter les champs d'analyse avec des indications spécifiques ou nécessaires à la gestion de l'aire protégée en examen. L'évaluation de l'importance des formes de gouvernance et de partenariats dans la gestion de l'aire protégée doit se faire selon les barèmes indiqués. Dans l'espace réservé aux commentaires, il est nécessaire de reporter la typologie de gouvernance de l'aire protégée définie au point CTX 1.2 du contexte d'intervention pour mieux évaluer la valeur et l'importance des formes de gouvernance et de partenariats adoptées par l'aire protégée.

Attention, cette section suscite généralement beaucoup de discussions, et peut prendre beaucoup de temps. II revient au coach assistant de canaliser les discussions.

\section{C1.2}

Contexte

Valeur et Importance : Sous-indicateur Classifications

Le point C1.2 permet d'estimer la valeur et l'importance du (des) statut(s) accordé à l'aire protégée au profit de la conservation au niveau 
national, régional ou international. L'importance des classifications de l'aire protégée doit se faire sur la base de la liste (indicative et non-exhaustive) des statuts spéciaux identifiés au point CTX 1.3 du contexte de l'intervention et des autres statuts possibles. L'estimation de l'importance attribuée aux classifications dans la gestion de l'aire protégée doit se faire selon les barèmes indiqués.

Il est possible d'avoir une valeur 0 lorsque la gestion du parc ne prend pas en compte une classification. Par ex., le site est classé Zone importante pour les oiseaux mais rien n'est fait en considération de ce statut. Attention, cela ne veut pas dire que la Zone importante pour les oiseaux n'est pas importante mais que la gestion du parc ne prend pas en compte la classification en Zone importante pour les oiseaux.

\section{C1.3}

Contexte

Valeur et Importance : Sous-indicateur Espèces animales et végétales phares, menacées, endémiques, exploitées, invasives, avec données insuffisantes

Le point C1.3 permet d'estimer le degré d'importance et la prise en compte dans le processus de gestion de l'aire protégée des espèces phares, menacées, endémiques, exploitées, invasives, avec données insuffisantes, pouvant être adoptées comme indicateurs écologiques d'excellence. L'évaluation doit se faire sur la base de la liste des espèces identifiées aux points : CTX 4.1 ; CTX 4.2 du contexte de l'intervention. L'estimation de l'importance attribuée aux espèces utilisées comme indicateurs dans la gestion de l'aire protégée doit se faire selon les barèmes indiqués.

Important : les espèces listées ici vont faire l'objet d'un suivi. II faut bien préciser ici qu'il s'agit d'actions spécifiques pour les espèces mentionnées.

\section{C1.4}

Contexte

Valeur et Importance : Sous-indicateur habitats terrestres et marins et couverture des sols et changement dans l'utilisation des sols (land-cover et land-change)

Le point C1.4 permet d'estimer le degré d'importance et la prise en compte, dans le processus de gestion de l'aire protégée, des habitats terrestres et marins et de l'utilisation et de la couverture des sols pouvant être adoptées comme indicateurs d'excellence. L'évaluation doit se faire sur la base de la liste des éléments identifiés au point CTX 4.3 du contexte d'intervention. L'estimation de l'importance attribuée aux habitats terrestres et marins et à l'utilisation et à la couverture des sols dans la gestion de l'aire protégée doit se faire selon les barèmes indiqués.

\section{C1.5}

Contexte

\section{Valeur et Importance : Sous-indicateur Changement Climatique}

Le point C1.5 permet d'estimer le degré d'importance et la prise en compte dans le processus de gestion de l'aire protégée des effets du changement climatique les plus significatifs et pouvant être adoptés comme indicateurs écologiques d'excellence pour suivre l'efficacité de l'atténuation et de l'adaptation des réponses au phénomène. L'analyse est facilitée par la présentation de la liste des catégories et l'attribution de l'importance par rapport à l'aire protégée des services écosystémiques adoptés au point CTX 6.1 du contexte de l'intervention. L'estimation de l'importance attribuée au maintien et à la valorisation des services écosystémiques dans la gestion de l'aire protégée doit se faire selon les barèmes indiqués.

\section{C1.6}

Contexte

Valeur et Importance : Sous-indicateur Espèces animales et végétales phares, menacées, endémiques, exploitées, invasives, avec données insuffisantes

Le point C1.6 permet d'estimer le degré d'importance et la prise en compte dans le processus de gestion de l'aire protégée des services écosystémiques les plus significatifs et pouvant être adoptés comme indicateurs écologiques. L'analyse est facilitée par la présentation de la liste des catégories et l'attribution de l'importance par rapport à l'aire protégée des services écosystémiques adoptés au point CTX 7.1 du contexte d'intervention. L'estimation de l'importance attribuée au maintien et à la valorisation des services écosystémiques dans la gestion de l'aire protégée doit se faire selon les barèmes indiqués.

\section{C2}

\section{Contraintes ou appuis externes}

Le point C2 permet d'estimer les contraintes ou les appuis et avantages exercés par l'environnement politico-institutionnel et social externe. L'analyse est soutenue par la suggestion des éventuelles contraintes ou appuis externes à la gestion de l'aire protégée. II est possible de compléter les champs d'analyse avec des indications spécifiques ou nécessaires à la gestion de l'aire protégée étudiée. L'évaluation doit se faire sur la base de la liste des 
contraintes, ou appuis externes pré-identifiés. II est possible de compléter les champs d'analyse avec des indications spécifiques ou nécessaires à la gestion de l'aire protégée étudiée. L'importance de ces contraintes ou appuis externes dans la gestion de l'aire protégée doit être identifiée, puis estimée en fonction de critères organisés selon les barèmes indiqués. L'analyse est facilitée avec l'utilisation du graphique "Contrainte - Support - Pouvoir des acteurs » (voir schéma 3), proposé à la page 58.

\section{C3}

\section{Menaces}

Le point C3 permet d'estimer le degré d'importance et la prise en compte dans le processus de gestion de l'aire protégée des menaces principales et pouvant être adoptées comme indicateurs d'excellence dans la gestion de l'aire protégée. L'analyse est facilitée par la présentation de la liste des catégories de menaces adoptées au point CTX 5.1 du contexte d'intervention. L'importance de ces menaces dans la gestion de l'aire protégée doit déjà être identifiée par l'utilisation très recommandée du Calculateur de menaces et les valeurs sont à reporter dans les catégories indiquées. La dernière version du Formulaire doit en principe reporter automatiquement les valeurs dans le tableau. Si l'analyse n'a pas voulu ou pu utiliser le Calculateur de menaces, les niveaux de menaces peuvent être estimés de manière indicative selon les barèmes indiqués.

À la fin du chapitre "Contexte de la gestion " du Formulaire, un tableau permet d'identifier des éléments importants à prendre en compte dans la gestion de l'aire protégée. Le tableau est organisé pour insérer :

- les lignes de base de l'état de conservation ;

- les conditions souhaitées ou à atteindre (objectif) ;

- et les valeurs de référence (benchmarks) à utiliser pour l'amélioration de la gestion.

Les valeurs définies peuvent être utilisées comme éléments importants pour la gestion et le suivi des activités de l'aire protégée, et plus spécifiquement pour les phases de planification, recherche des ressources (intrants), processus, détermination des résultats et des objectifs d'impact.

\section{PLANIFICATION}

P1

Planification

\section{Adéquation des dispositions législatives et réglementaires}

Le point P1 permet d'estimer le niveau d'adéquation ou d'appropriation des réglementations en vigueur pour le contrôle et l'utilisation des terres et des ressources naturelles (par ex. la cueillette) dans l'aire protégée (l'analyse prend aussi en compte les aires protégées avec des droit coutumiers et celles avec gestion des ressources naturelles). L'analyse est soutenue par une liste des dispositions législatives et réglementaires possibles qui interviennent dans, ou influencent, la gestion de l'aire protégée. II est possible de compléter les champs d'analyse avec des indications spécifiques ou nécessaires à la gestion de l'aire protégée en examen. L'adéquation ou la faiblesse des réglementations en vigueur par rapport aux exigences de gestion doivent être estimées selon les barèmes indiqués.

Planification

\section{Conception et configuration de l'aire protégée}

Le point P2 permet d'estimer si la conception et la configuration de l'aire protégée assurent la protection de ses valeurs et les aspects importants pour laquelle elle a été créée. Plus précisément, nous devons estimer si la taille et la forme de l'aire protégée sont convenables pour protéger les espèces, les habitats et assurer les fonctionnements naturels comme les processus écologiques, les captages d'eau, etc. II est également demandé d'estimer la configuration de l'aire protégée à travers l'indice de forme (surface/contour soit km²/ $\mathrm{km}$ ) afin de prendre en considération les difficultés

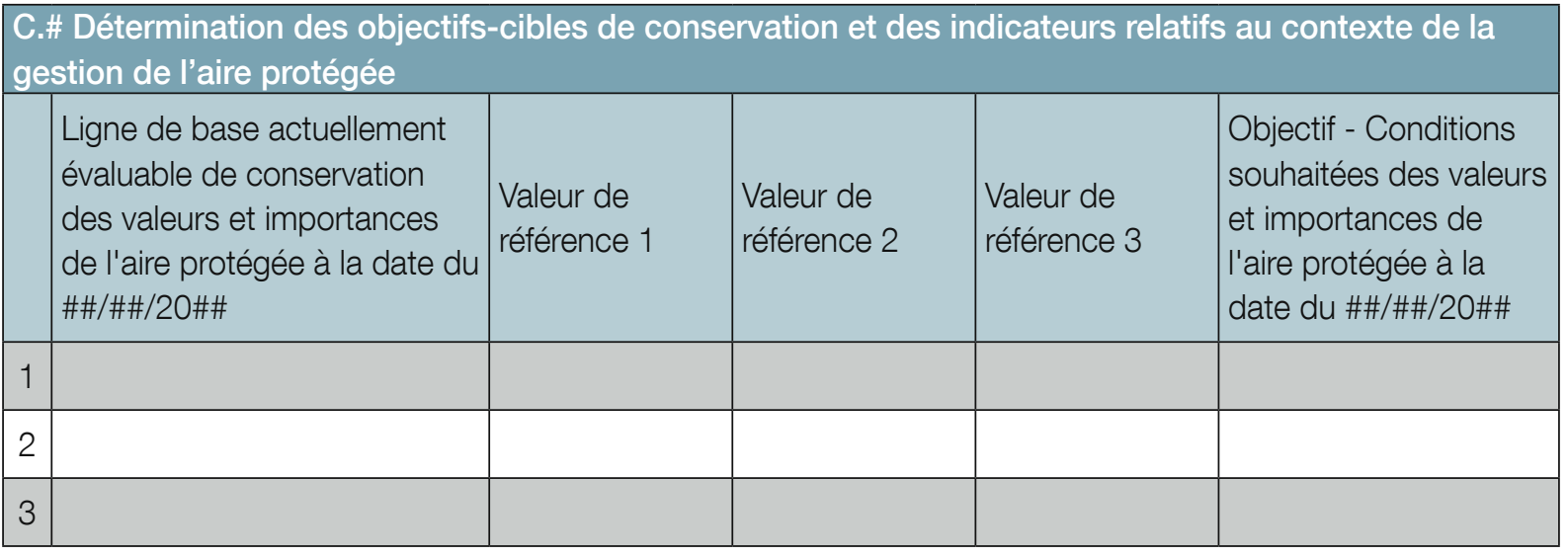


à assurer le contrôle de l'aire protégée. L'analyse est facilitée par la présentation de la liste des catégories des aspects liés aux caractéristiques géophysiques de l'aire protégée. II est possible de compléter les champs d'analyse avec des indications spécifiques ou nécessaires à la gestion de l'aire protégée étudiée. L'adéquation de la conception et des caractéristiques géophysiques de l'aire protégée pour la gestion et la protection des valeurs de l'aire protégée doit être estimée en fonction de critères positifs ou négatifs selon les barèmes indiqués.

\section{P3}

\section{Démarcation de l'aire protégée}

Le point P3 analyse le degré de connaissances et de signalisation des limites de l'aire protégée quant aux dispositions de gestion à adopter pour assurer la protection de ses valeurs importantes. L'analyse est facilitée par la proposition de cinq cas de figure (valeur « 4 " seulement dans le cas où les limites sont parfaitement connues et signalés à 100\%) par rapport à la connaissance des limites et à leur signalisation. $\mathrm{Si}$ les gestionnaires de l'aire protégée connaissent exactement le pourcentage du périmètre délimité de l'aire protégée, cette valeur doit être enregistrée. Dans le cas contraire, il est nécessaire de choisir une des quatre catégories indiquées dans le barème relatif.

\section{P4}

Planification

\section{Plan de gestion}

Le point P4 analyse l'existence et l'application du plan de gestion de l'aire protégée. L'examen est facilité par la proposition d'une double analyse, facilitée par la proposition de quatre cas de figure pour chaque estimation. La première analyse concerne l'élaboration, l'approbation et la mise en œuvre du plan de gestion. La deuxième analyse concerne la qualité du plan relativement à la définition d'une vision, une mission et des objectifs clairs et bien ciblés. Chaque analyse est facilitée par quatre cas de figure. II est donc nécessaire de choisir une des quatre catégories indiquées dans le barème relatif. Étant donné la complexité de la question, il faudra inviter les évaluateurs internes à préciser les aspects les plus importants relatifs à l'élaboration, à l'adoption et à la mise en œuvre du plan de gestion dans l'espace dédié aux observations.

\section{P5}

\section{Plan de travail}

Le point P5 analyse l'existence et l'application du plan de travail (annuel ou pluriannuel) de l'aire protégée. L'examen est facilité par la proposition d'une double analyse facilitée par la proposition de quatre cas de figure pour chaque estimation. Il est donc nécessaire de choisir une des quatre catégories indiquées dans le barème relatif. La première analyse est facilitée par la proposition de quatre cas de figure par rapport à l'existence, au niveau de mise en œuvre des interventions programmées et à l'exécution des activités sur la base des objectifs du plan de travail. La deuxième analyse concerne la correspondance des interventions et des cibles de conservation/ gestion par rapport à l'orientation donnée par le plan de gestion (vision - mission - objectifs). Étant donné la complexité de la question, il faudra inviter les évaluateurs internes à préciser les aspects les plus importants relatifs à l'élaboration, à l'adoption et à la mise en œuvre du plan de travail annuel ou pluriannuel dans l'espace dédié aux observations.

\begin{tabular}{|c|c|c|c|c|c|}
\hline & $\begin{array}{l}\text { Détermination des objectifs } \\
\text { til de planification de l'aire }\end{array}$ & $\begin{array}{l}\text {-cibles de c } \\
\text { jrotégée }\end{array}$ & rvation et & idicateur & itifs aux exercices et \\
\hline & $\begin{array}{l}\text { Ligne de base actuellement } \\
\text { évaluable de l'adéquation } \\
\text { des dispositions législatives, } \\
\text { configuration de l'AP et } \\
\text { de l'élaboration des outils } \\
\text { de gestion à la date du } \\
\text { \#\#/\#\#/20\#\# }\end{array}$ & $\begin{array}{l}\text { Valeur de } \\
\text { référence } 1\end{array}$ & $\begin{array}{l}\text { Valeur de } \\
\text { référence } 2\end{array}$ & $\begin{array}{l}\text { Valeur de } \\
\text { référence } 3\end{array}$ & $\begin{array}{l}\text { Objectif - Conditions } \\
\text { souhaitées de } \\
\text { l'adéquation } \\
\text { des dispositions } \\
\text { législatives, } \\
\text { configuration de l'AP } \\
\text { et de l'élaboration } \\
\text { des outils de } \\
\text { gestion à la date du } \\
\text { \#\#/\#\#/20\#\# } \\
\end{array}$ \\
\hline 1 & & & & & \\
\hline 2 & & & & & \\
\hline 3 & & & & & \\
\hline
\end{tabular}


P6

Planification

\section{Objectifs de l'aire protégée}

Le point P6 permet d'évaluer si la gestion de l'aire protégée est effectuée en fonction d'objectifs correctement identifiés par des indicateurs et des valeurs de référence pertinents pour assurer la conservation des valeurs et l'importance de l'aire protégée. Pour vérifier ce dernier point, on doit analyser la correspondance entre les objectifs de gestion, les documents de programmation et l'état du contexte. L'analyse est facilitée par la présentation des catégories d'éléments clé de la gestion et la gouvernance d'une aire protégée. L'estimation doit prendre en compte la correspondance entre valeurs et objectifs selon les barèmes indiqués. Étant donné la complexité de la question, il faudra inviter les évaluateurs internes à préciser les aspects les plus importants relatifs à l'adoption des objectifs de gestion dans l'espace dédié aux observations.

À la fin du chapitre "Planification " du Formulaire, un tableau permet d'identifier les éléments importants à prendre en compte dans la gestion de l'aire protégée. Le tableau est organisé pour insérer :

- les lignes de base de l'état de conservation ;

- les conditions souhaitées l'état de conservation favorable ou à atteindre (objectif) ;

- et les valeurs de référence (benchmarks) à utiliser pour l'amélioration de la gestion.

Les valeurs définies peuvent être utilisées comme éléments importants pour la gestion et le suivi des activités de l'aire protégée, et plus spécifiquement pour les phases de planification, recherche des ressources (intrants), processus, détermination des résultats et des objectifs d'impact.

\section{INTRANTS}

\section{I1}

\section{Informations de base}

Le point I1 permet analyser le niveau des informations fondamentales par rapport aux exigences de gestion de l'aire protégée. L'analyse impose de lister les éléments disposant ou non d'informations fondamentales pour assurer la gestion de l'aire protégée sur la base des thématiques du contexte d'intervention, points CTX 4 ; CTX 5 ; CTX 6 et CTX 7. II est possible de compléter les champs d'analyse avec des indications spécifiques ou nécessaires à la gestion de l'aire protégée étudiée. Le niveau d'informations de base disponibles pour chaque élément doit être estimé selon les barèmes indiqués.

\begin{tabular}{cc}
12 & Intrants \\
\hline Personnel &
\end{tabular}

Le point 12 permet d'analyser l'adéquation des effectifs des ressources humaines par rapport aux exigences de gestion de l'aire protégée. L'analyse est facilitée par l'insertion manuelle ou automatique (selon la version du logiciel exploité) de la liste des catégories du personnel adoptée au point CTX 3.1 du contexte de l'intervention. Le niveau d'adéquation des effectifs par rapport aux besoins de gestion de l'aire protégée doit être estimé selon les barèmes indiqués.

\section{3}

Intrants

\section{Budget actuel}

Le point 13 assure une réflexion concernant la disponibilité des ressources financières par rapport aux exigences de conservation de l'aire protégée. L'évaluation est facilitée par l'analyse des ressources financières au point CTX 3.2 du contexte de l'intervention. Si les gestionnaires de l'aire protégée ont connaissance de l'exacte disponibilité des ressources financières, cette valeur doit être enregistrée. Dans le cas contraire, pour l'évaluation, il est nécessaire de choisir une des quatre catégories indiquées dans le barème relatif. Étant donné la complexité de la question, il faudra inviter les évaluateurs internes à préciser les aspects les plus importants relatifs à la disponibilité des ressources financières dans l'espace dédié aux observations.

\section{4}

Intrants

\section{Sécurisation du budget}

Le point 14 estime le niveau de sécurisation du budget par rapport aux exigences de conservation de l'aire protégée. L'analyse est facilitée par l'analyse des ressources financières au point CTX 3.2.4 du contexte de l'intervention et par la proposition de quatre cas de figure par rapport à la sécurisation budgétaire annuelle ou pluriannuelle et des sources de financement. Pour l'évaluation, il est nécessaire de choisir une des quatre catégories indiquées dans le barème relatif. Étant donné la complexité de la question, il faudra inviter les évaluateurs internes à préciser les aspects les plus importants relatifs à la sécurisation du budget dans l'espace dédié aux observations.

\section{5}

Intrants

\section{Infrastructures, équipements et installations}

Le point 15 évalue l'adéquation des infrastructures, équipements et installations par rapport aux 


\begin{tabular}{|l|l|l|l|l|l|}
\hline \multicolumn{7}{|l|}{$\begin{array}{l}\text { \#. Détermination des objectifs-cibles de conservation et des indicateurs relatifs aux intrants } \\
\text { nécessaires pour la mise en ouvre de la planification de l'aire protégée }\end{array}$} \\
\hline \multicolumn{2}{|l|}{$\begin{array}{l}\text { Ligne de base actuellement } \\
\text { évaluable des intrants à la } \\
\text { date du \#\#/\#\#/20\#\# }\end{array}$} & $\begin{array}{l}\text { Valeur de } \\
\text { référence 1 }\end{array}$ & $\begin{array}{l}\text { Valeur de } \\
\text { référence 2 }\end{array}$ & $\begin{array}{l}\text { Valeur de } \\
\text { référence 3 }\end{array}$ & $\begin{array}{l}\text { Objectif - Conditions } \\
\text { souhaitées des } \\
\text { intrants à la date du } \\
\# \# / \# \# / 20 \# \#\end{array}$ \\
\hline 1 & & & & & \\
\hline 2 & & & & & \\
\hline 3 & & & & & \\
\hline
\end{tabular}

exigences de gestion de l'aire protégée. L'analyse est facilitée par la présentation de la liste des catégories des infrastructures, équipements et installations adoptée au point CTX 3.3 du contexte de l'intervention. Le niveau d'adéquation des infrastructures, équipements et installations par rapport aux exigences de gestion de l'aire protégée doit être estimé selon les barèmes indiqués.

À la fin du chapitre "Intrants " du Formulaire, un tableau permet d'identifier les éléments importants à prendre en compte dans la gestion de l'aire protégée. Le tableau est organisé pour insérer :

- les lignes de base de l'état de conservation ;

- les conditions souhaitées l'état de conservation favorable ou à atteindre (objectif) ;

- et les valeurs de référence (benchmarks) à utiliser pour l'amélioration de la gestion.

Les valeurs définies peuvent être utilisées comme éléments importants pour la gestion et le suivi des activités de l'aire protégée et, plus spécifiquement, pour les phases de planification, recherche des ressources (intrants), processus, détermination des résultats et des objectifs d'impact.

\section{PROCESSUS}

\section{Systèmes et processus de gestion interne}

\section{PR1}

\section{Capacités et formation du personnel}

Le point PR1 évalue les compétences par rapport au rôle et aux fonctions attribuées, et l'adéquation en formation professionnelle du personnel par rapport aux exigences de gestion de l'aire protégée. L'analyse est facilitée par l'insertion manuelle ou automatique (selon la version du logiciel exploité) du personnel identifié au point CTX 3.1 du contexte de l'intervention et évalue le niveau de compétence et de formation du personnel disponible par rapport au poste de service et aux plus importantes thématiques de gestion de l'aire protégée. Le niveau moyen de compétence et de formation du personnel disponible par rapport aux exigences de gestion doit être estimé en fonction de critères organisés selon les barèmes indiqués.

\section{PR2}

Processus

\section{Politiques et procédures de gestion des ressources humaines}

Le point PR2 analyse l'adéquation des politiques et des procédures de gestion des ressources humaines par rapport aux exigences de gestion de l'aire protégée. L'analyse s'appuie sur la liste des conditions indispensables pour avoir une bonne politique et des procédures de gestion des ressources humaines adéquates dans une aire protégée. II est possible de compléter les champs d'analyse avec des indications spécifiques ou nécessaires à la gestion de l'aire protégée étudiée. L'estimation de la conduite d'une bonne politique et des procédures correctes de gestion des ressources humaines doivent être estimées en fonction des barèmes indiqués.

\section{PR3}

Processus

\section{Systèmes et processus de gestion des ressources humaines}

Le point PR3 permet d'estimer le degré de motivation ou l'aptitude au travail du personnel de service de l'aire protégée. L'analyse s'appuie sur la liste des conditions indispensables pour entretenir un bon niveau de motivation du personnel. II est possible de compléter les champs d'analyse avec des indications spécifiques ou nécessaires à la gestion de l'aire protégée étudiée. Les conditions indispensables pour entretenir un bon niveau de motivation du personnel dans une aire protégée doivent être estimées en fonction des barèmes indiqués.

\section{PR4}

Processus

\section{Management et leadership interne}

Le point PR4 évalue le niveau de gouvernance interne et de leadership. Plus spécifiquement, il apporte une évaluation de l'exécution des ordres et la discipline du personnel et le processus et la 
prise de décision par les responsables pour assurer une bonne gestion de l'aire protégée. Compte tenu de la difficulté de cette analyse, l'évaluation comporte une double notation selon le barème : $0-1-2-3$. Il est donc nécessaire de choisir le barème relatif le plus représentatif de la réalité dans chacune des deux séries de quatre catégories.

Section un peu délicate, car c'est l'évaluation du leadership interne donc de la chaîne de commandement ou de prise de décision.

\section{PR5}

Processus

\section{Gestion comptable et financière}

Le point PR5 permet d'analyser l'efficacité comptable et financière, y compris budgétaire des ressources financières disponibles pour couvrir les besoins essentiels de gestion de l'aire protégée. L'analyse est facilitée par la proposition de quatre cas de figure par rapport à la relation entre le degré de gestion comptable et financière et son impact sur l'efficacité de la gestion de l'aire protégée. L'efficacité de la gestion comptable et financière pour assurer le bon fonctionnement de l'aire protégée doit être estimée en fonction des barèmes indiqués. Étant donné la complexité de la question, les évaluateurs internes sont invités à préciser les aspects les plus importants relatifs à l'adoption des objectifs de gestion, dans l'espace dédié aux observations.

\section{PR6}

Processus

\section{Entretien des infrastructures, des équipements et des installations}

Le point PR6 permet de déterminer le niveau d'attention porté sur l'entretien des infrastructures, des équipements et des installations comme opérations importantes en appui à la gestion de l'aire protégée. L'analyse est facilitée par la présentation de la liste des infrastructures, des équipements et des installations adoptés au point CTX 3.3 du contexte de l'intervention. Le degré d'entretien des infrastructures, des équipements et des installations par rapport aux exigences de gestion doit être identifié, puis estimé en fonction de critères organisés selon les barèmes indiqués. Étant donné la complexité de la question, les évaluateurs internes devront préciser les aspects les plus importants relatifs aux entretiens des infrastructures, des équipements et des installations dans l'espace dédié aux observations.

\section{Protection/gestion}

\section{PR7}

Processus

Gestion des valeurs et des aspects importants de l'aire protégée

Le point PR7 permet d'analyser l'existence et le niveau d'efficacité des mesures actives adoptées par l'aire protégée au profit de la gestion active des espèces et des habitats, des processus écologiques, des ressources naturelles, des menaces, des effets du changement climatique, des services écosystémiques, etc. L'analyse impose de lister les éléments de gestion active les plus importants sur la base des éléments du contexte d'intervention, points CTX 4 et CTX 5 (les éléments relatifs aux CTX 6 et CTX 7 sont examinés dans les points PR 18 et PR 19). Pour la gestion active, on considère les activités autre que le contrôle, la protection, le suivi et la recherche au profit des valeurs de l'aire protégée (par ex. réalisation de points d'eau, d'infrastructures d'intervention même à l'extérieur de l'aire protégée comme l'atténuation des menaces, du changement climatique, etc. à la seule condition qu'elles soient liées aux valeurs et aux aspects importants de l'aire protégée). Pour faciliter l'analyse, une double liste est prévue : i) les mesures actives de gestion et ii) les cibles de gestion. Le niveau, l'existence et les mesures actives adoptées pour chaque valeur et l'importance de l'aire protégée doivent être estimés selon les barèmes indiqués. Étant donné la complexité de la question, les évaluateurs internes sont invités à préciser les aspects les plus importants relatifs à la gestion des valeurs et les aspects essentiels de l'aire protégée dans l'espace dédié aux observations.

Mots clés ici : mesure active c'est-à-dire qu'il s'agit d'actions volontaires impliquant la mobilisation de temps, de personnel ou de moyens pour assurer la gestion des valeurs du parc.

\section{PR8}

Processus

\section{Systèmes de protection des valeurs et des aspects importants de l'aire protégée}

Le point PR8 permet d'analyser le degré d'efficacité de la protection et de la conservation du patrimoine en biodiversité, les valeurs et les éléments importants de l'aire protégée. L'analyse impose de lister les éléments de gestion les plus importants sur la base des valeurs et des éléments d'importance listés dans le contexte 
d'intervention, points CTX 4 ; CTX 5 et CTX 7. Le degré d'efficacité de la protection et de la limitation à l'accès au patrimoine en biodiversité et aux ressources naturelles et culturelles de l'aire protégée doit être estimé selon les barèmes indiqués. Étant donné la complexité de la question, les évaluateurs internes sont invités à préciser les aspects les plus importants relatifs à la gestion des valeurs et les aspects essentiels de l'aire protégée dans l'espace dédié aux observations.

\section{PR9}

\section{Contrôle de l'aire protégée}

Le point PR9 permet d'analyser le niveau de contrôle territorial de l'aire protégée. L'analyse est facilitée par la proposition de cinq cas de figure (valeur « 4 " seulement dans le cas du contrôle de $100 \%$ de la surface de l'aire protégée) par rapport au niveau de contrôle de l'aire protégée. Si les gestionnaires de l'aire protégée ont la connaissance exacte de la valeur du contrôle de la surface de l'aire protégée (voir point CTX 2.3), cette valeur doit être enregistrée. Dans le cas contraire, il est nécessaire de choisir une des quatre catégories indiquées dans le barème relatif.

\section{PR10}

Processus

\section{Application des lois}

Le point PR10 permet d'analyser le niveau et les capacités d'application des lois et des réglementations qui régissent la gestion de l'aire protégée. L'analyse s'appuie sur une liste des cas d'application des lois et des réglementations. I est possible de compléter les champs d'analyse avec des indications spécifiques ou nécessaires à la gestion de l'aire protégée étudiée. Les cas d'application des lois et des réglementations pour assurer la bonne gestion de l'aire protégée doivent être estimés en fonction des barèmes indiqués.

\section{Relations avec les parties prenantes}

\section{PR11}

Processus

Implication des communautés, des ayants droit et des parties prenantes

Le point PR11 estime le degré d'implication des communautés locales, des ayants droit et des parties prenantes dans les décisions de gestion de l'aire protégée. L'analyse s'appuie sur une liste indicative des ayants droit principaux et plus fréquents dans les relations avec les aires protégées. II est possible de compléter la liste avec tous les ayants droit qui collaborent avec l'aire protégée étudiée. Le niveau d'implication des communautés, des ayants droit et des parties prenantes dans les décisions de gestion de l'aire protégée doit être estimé en fonction des barèmes indiqués.

PR12 Processus Avantages/assistance appropriés au profit des collectivités

Le point PR12 évalue l'état, l'adéquation et les effets des activités et des programmes en cours dans l'aire protégée visant des avantages ou l'assistance appropriés vis-à-vis des collectivités riveraines de l'aire protégée. L'analyse s'appuie sur la suggestion des activités les plus courantes et des programmes initiés par les aires protégées au profit des collectivités riveraines de l'aire protégée. II est possible de compléter les champs d'analyse avec d'autres activités initiées par l'aire protégée étudiée. L'état, l'adéquation et les effets des activités et des programmes en cours dans l'aire protégée visant des avantages ou l'assistance appropriée au profit des collectivités, doivent être estimés en fonction des barèmes indiqués.

PR13

Processus

\section{Relations avec les acteurs}

Le point PR12 évalue l'état, l'adéquation et les effets des programmes d'éducation environnementale (connaissance de la nature) et d'éducation à l'environnement (aptitude à une gestion durable des ressources naturelles) généralistes ou spécifiquement liées aux objectifs et aux besoins de la conservation et de la gestion des ressources naturelles de l'aire protégée. L'analyse s'appuie sur une liste des activités les plus courantes d'éducation environnementale liées aux objectifs et nécessités de la conservation et de la gestion des ressources naturelles. II est possible de compléter les champs d'analyse avec des programmes et activités spécifiques ou nécessaires d'éducation environnementale ou à l'environnement de l'aire protégée étudiée. L'état, l'adéquation et les effets des activités et des programmes d'éducation environnementale ou à l'environnement, généralistes ou spécifiquement liés aux objectifs et nécessités de la conservation et de la gestion des ressources naturelles au profit de la gestion de l'aire protégée, doivent être estimés en fonction des barèmes indiqués.

\section{Gestion du tourisme}

PR14

Processus

\section{Gestion des visiteurs}

Le point PR14 analyse la mise en application des conditions nécessaires pour avoir une bonne adéquation des installations et des services au profit 
du tourisme et de l'éducation environnementale. L'analyse concerne exclusivement les aires protégées qui sont en mesure d'assurer la valorisation du patrimoine naturel. L'analyse s'appuie sur la suggestion des conditions nécessaires pour avoir une bonne adéquation des installations et des services pour les visiteurs dans une aire protégée (tourisme et éducation environnementale). II est possible de compléter les champs d'analyse avec des indications spécifiques ou nécessaires à la gestion touristique dans l'aire protégée en examen. Le niveau d'application des conditions nécessaires pour avoir une bonne adéquation des installations et des services pour les visiteurs dans l'aire protégée doit être estimé en fonction des barèmes indiqués.

\section{PR15}

Processus

\section{Visiteurs et impacts}

Le point PR15 permet d'analyser les dispositions de prise en charge et d'atténuation des impacts des activités touristiques de manière appropriée pour assurer le maintien des valeurs et de l'importance de l'aire protégée. L'analyse s'appuie sur la suggestion des conditions nécessaires pour la prise en charge et l'atténuation des impacts des visites touristiques dans l'aire protégée. II est possible de compléter les champs d'analyse avec des indications spécifiques ou nécessaires à la gestion touristique dans l'aire protégée en examen. Le niveau d'application des dispositions pour la prise en charge et l'atténuation des impacts des visites touristiques dans l'aire protégée doit être estimé en fonction des barèmes indiqués.

\section{Suivi et recherche}

\section{PR16}

Processus

\section{Systèmes de suivi des valeurs et des aspects importants de l'aire protégée}

Le point PR16 analyse l'état, l'adéquation et les effets des activités de suivi des valeurs et des aspects importants de l'aire protégée par rapport aux efforts de gestion et à l'ampleur et à la gravité des menaces. L'analyse s'appuie sur une liste des conditions les plus importantes pour effectuer un suivi pertinent des efforts de gestion et des menaces portées aux valeurs et aux aspects importants de l'aire protégée (points CTX 4 ; CTX 5 ; CTX 6 et CTX 7). II est possible de compléter les champs d'analyse avec des programmes et activités de suivi spécifiques ou nécessaires à la gestion de l'aire protégée en examen. L'état, l'adéquation et les effets des activités et des programmes de suivi des valeurs et des aspects importants liés aux objectifs et besoins de la conservation et de la gestion des ressources naturelles de l'aire protégée doivent être estimés en fonction des barèmes indiqués.
PR17

Processus

Recherche et biosuivi (biomonitoring)

Le point PR17 évalue l'état, l'adéquation et les effets des activités de recherche et de biosuivi en appui à la gestion des valeurs et des aspects essentiels de l'aire protégée. L'analyse impose de lister les éléments de recherche et de biosuivi liés à l'amélioration de la conservation et de la gestion des valeurs les plus importantes, à identifier sur la base des éléments du contexte d'intervention, points CTX 4 ; CTX 5 ; CTX 6 et CTX 7. Les activités de recherche et de biosuivi en appui à la gestion des valeurs et des aspects essentiels de l'aire protégée doivent être identifiées, puis évaluées en fonction de critères d'efficacité sur la base des besoins de gestion de l'aire protégée selon les barèmes indiqués.

\section{Gestion des effets du changement climatique et des services écosystémiques}

PR18

Processus

\section{Gestion des effets du changement climatique}

Le paramètre PR18 permet d'évaluer les dispositions d'atténuation et d'adaptation des effets du changement climatique dans la planification et la gestion de l'aire protégée. L'analyse s'appuie sur une liste des dispositions d'atténuation et d'adaptation au changement climatique dans la planification et la gestion sur la base des éléments du contexte d'intervention, points CTX 6.1 et CTX 6.2. L'existence et l'évaluation des activités/programme d'atténuation et adaptation des effets du changement climatique dans la planification et la gestion des aspects naturels et culturels doivent être estimées en fonction de critères organisés selon les barèmes indiqués.

\section{PR19}

Processus

\section{Services écosystémiques}

Le paramètre PR19 permet d'évaluer l'état, l'adéquation et les effets des activités des actions et des programmes en faveur de la conservation et de la valorisation des services écosystémiques rendus par l'aire protégée pour le bien-être humain. Il est important de noter que l'attribution du caractère légal ou illégal de l'approvisionnement dépend de la catégorie de classification de l'aire protégée et des éventuelles utilisations coutumières tolérées, autorisées ou légalisées à l'intérieur du domaine classé. L'analyse impose de lister les services écosystémiques réellement rendus par l'aire protégée sur la base des éléments du contexte d'intervention, point CTX 7.1 et CTX 7.2. L'importance des interventions de l'aire protégée pour le maintien et la valorisation des services écosystémiques fournis pour le bien-être humain doivent être estimées selon les barèmes indiqués. 


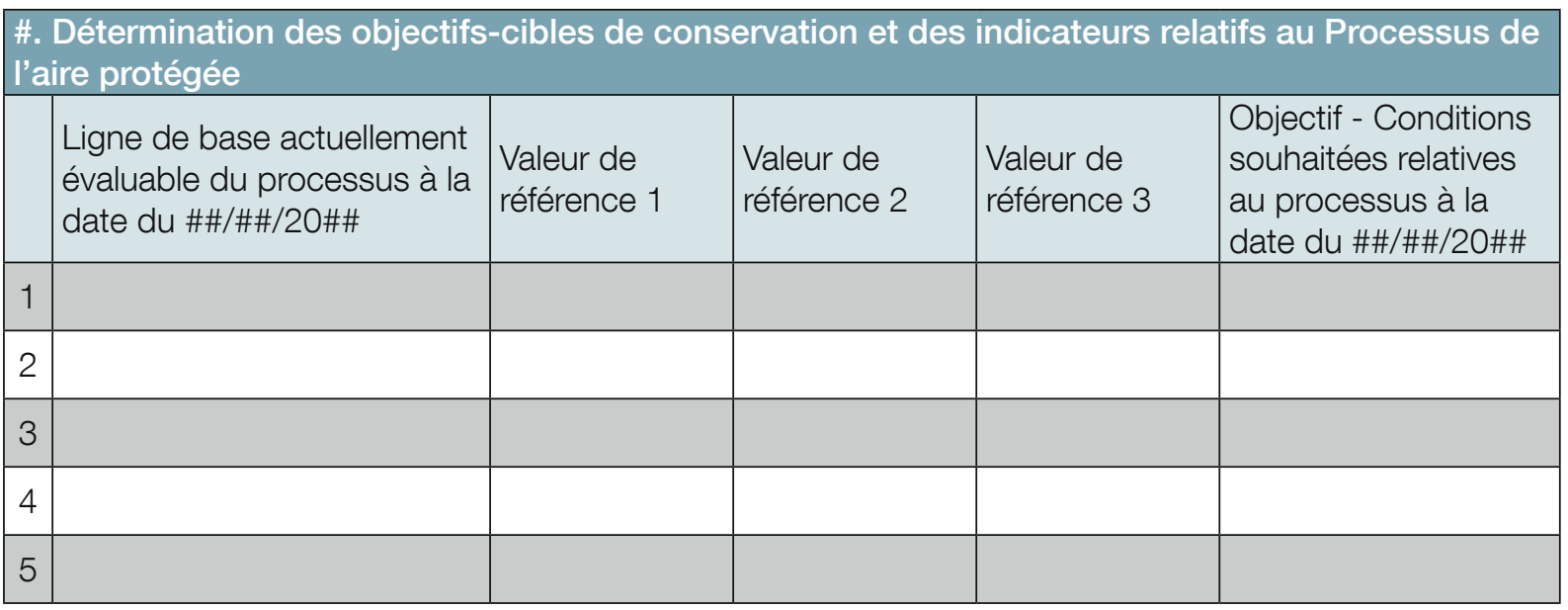

À la fin du chapitre "Processus " du Formulaire, un tableau permet d'identifier les éléments importants à prendre en compte dans la gestion de l'aire protégée. Le tableau est organisé pour insérer :

- les lignes de base de L'état de conservation ;

- les conditions souhaitées L'état de conservation favorable ou à atteindre (objectif) ;

- et les valeurs de référence (benchmarks) à utiliser pour l'amélioration de la gestion.

Les valeurs définies peuvent être utilisées comme éléments importants pour la gestion et le suivi des activités de l'aire protégée et plus spécifiquement pour les phases de planification, recherche des ressources (intrants), processus, détermination des résultats et des objectifs d'impact.

\section{RÉSULTATS (Outputs)}

\section{R1}

Résultats

\section{Mise en œuvre des principales activités du programme de travail annuel ou pluriannuel}

Le point R1 permet d'analyser la mise en œuvre du plan de travail annuel (ou pluriannuel). Le questionnaire propose de lister au maximum cinq activités principales du plan de travail pour évaluer leur mise en œuvre. La meilleure solution est de reporter les mesures envisagées par le plan de travail annuel (ou pluriannuel) accompagnées des valeurs de mise en œuvre attendus. Les activités du plan de travail doivent être listées, puis estimées sur la base de leur mise en œuvre selon les barèmes indiqués. Étant donné la complexité de la question, les évaluateurs internes sont invités à préciser les aspects les plus importants relatifs à la mise en œuvre des activités envisagées dans la programmation annuelle du travail de l'aire protégée dans l'espace dédié aux observations.

\section{$\mathrm{R} 2$}

Résultats

Réalisation des principaux résultats attendus du plan de travail annuel ou pluriannuel
Le point R2 permet d'estimer dans quelle proportion l'aire protégée a atteint les principaux résultats du plan de travail annuel (ou pluriannuel). Le questionnaire propose de lister au maximum cinq résultats principaux du plan de travail pluriannuel pour évaluer la part de réalisation estimée. La meilleure solution est de reporter les résultats attendus par le plan de travail annuel (ou pluriannuel) accompagnés des valeurs des résultats attendus. Les résultats du plan travail doivent être listés, puis leur degré de mise en œuvre estimé selon les barèmes indiqués. Étant donné la complexité de la question, les évaluateurs internes sont invités à préciser les aspects les plus importants relatifs à la mise en œuvre des résultats envisagés dans la programmation du travail de l'aire protégée dans l'espace dédié aux observations.

\section{EFFET/IMPACT (Outcomes)}

\section{E/l1}

Effets et impact

\section{Réalisation des objectifs de conservation}

Le point E/11 cherche à estimer dans quelle mesure l'aire protégée a atteint les objectifs du plan de gestion (ou du plan de travail pluriannuel en l'absence de plan de gestion) auquel il se réfère. Le questionnaire propose de lister cinq objectifs principaux (ou plus) du plan de travail pluriannuel ou du plan de gestion, afin d'évaluer l'impact atteint par les efforts de gestion de l'aire protégée. La meilleure solution serait de rapporter tous les objectifs fixés par le plan de travail pluriannuel ou le plan de gestion, accompagnés des valeurs de référence attendues, de la condition souhaitée ou de l'état de conservation souhaitée par rapport à un niveau de base. L'analyse impose que le plan de travail pluriannuel et le plan de gestion disposent d'indicateurs d'impact ou, d'une manière plus simple, de valeurs de référence à atteindre par les interventions. Les objectifs du plan de travail pluriannuel ou du plan de gestion doivent être listés, puis le degré de réalisation estimé selon 
les barèmes indiqués. Étant donné la complexité de la question, les évaluateurs internes sont invités à préciser les aspects les plus importants relatifs à la réalisation des objectifs identifiés dans le plan de travail pluriannuel ou le plan de gestion de l'aire protégée dans l'espace dédié aux observations.

\section{$E / 12$}

Effets et impact

\section{État de conservation des valeurs désignées de l'aire protégée}

Le point E/l2 se propose, en complément de l'évaluation de la réalisation des objectifs, d'estimer l'état de conservation des valeurs désignées de l'aire protégée. Le questionnaire propose de lister les principales valeurs de l'aire protégée utilisées comme indicateurs. L'analyse s'appuie sur la liste des catégories, et la meilleure solution serait de rapporter toutes les valeurs les plus importantes sur la base des éléments du contexte d'intervention aux points CTX 4 ; CTX 5 ; CTX 6 et CTX 7 . Cette évaluation est possible si la planification rapporte les valeurs de référence attendues, la condition souhaitée ou l'état de conservation souhaité par rapport à un état de conservation de base (baseline). Les valeurs désignées de l'aire protégée doivent être listées, puis l'état de conservation estimé en fonction de critères positifs ou négatifs selon les barèmes indiqués. Étant donné la complexité de la question, les évaluateurs internes sont invités à préciser les aspects les plus importants relatifs à l'état de conservation des valeurs désignées de l'aire protégée, dans l'espace relatif aux observations. On rappelle que l'état de conservation se réfère à la condition de la valeur examinée au moment de l'évaluation et dont l'analyse qui suivra (voir point E/ 13) estimera la tendance. Selon ces critères, l'état de conservation d'une aire protégée peut avoir une valeur négative, mais afficher quand même une tendance positive, et vice-versa.

\section{$E / 13$}

Effets et impact

\section{Tendances de l'état de conservation des} valeurs désignées de l'aire protégée

Le point E/I3 se propose, en complément de l'évaluation de la réalisation des objectifs et de l'état de conservation, d'estimer la tendance des valeurs désignées de l'aire protégée. Le questionnaire propose de lister les principales valeurs de l'aire protégée utilisées comme indicateurs. L'analyse s'appuie sur la liste des catégories, et la meilleure solution serait de rapporter toutes les valeurs les plus importantes sur la base des éléments du contexte d'intervention aux points CTX 4 ; CTX 5 ; CTX 6 et CTX 7 et précédemment analysés dans le point E/ 12. Les valeurs désignées de l'aire protégée doivent être listées, puis la tendance estimée en fonction de critères positifs ou négatifs selon les barèmes indiqués. Étant donné la complexité de la question, les évaluateurs internes sont invités à préciser les aspects les plus importants relatifs à la tendance des valeurs désignées de l'aire protégée, dans l'espace dédié aux observations. On rappelle que la tendance se réfère à l'évolution des valeurs par rapport à leur état antérieur, et que l'état de conservation se réfère à la condition au moment de l'évaluation analysée dans le point E/l2. Selon ces critères, la tendance d'une valeur d'une aire protégée peut être positive même si l'état de conservation a une valeur négative, et vice-versa.

\section{$E / 14$}

Effets et impact

\section{Effets et impact sur les communautés locales}

Le point E/14 analyse les effets et l'impact de la gestion de l'aire protégée sur les communautés locales en ce qui concerne les avantages et les inconvénients économiques. Le questionnaire propose de lister les activités ayant un impact économique positif ou négatif sur les communautés locales comme conséquence de la gestion de l'aire protégée. La meilleure solution est de lister toutes les actions les plus importantes rapportées dans le point PR12 de l'Évaluation de la gestion de l'aire protégée. À ce niveau, l'analyse est facilitée par la liste des activités et des programmes les plus couramment entrepris par les aires protégées et visant des avantages/assistance appropriés vis-à-vis des collectivités. Les activités et les programmes de gestion de l'aire protégée au profit des communautés locales doivent être listés, puis leurs effets et impacts économiques estimés en fonction de critères positifs ou négatifs selon les barèmes indiqués. Étant donné la complexité de la question, les évaluateurs internes sont invités à préciser les aspects les plus importants relatifs aux effets et à l'impact économique de la gestion de l'aire protégée sur les communautés locales dans l'espace dédié aux observations.

\section{E/15}

Effets et impact

\section{Effets et impact de l'atténuation et de l'adaptation au changement climatique}

Le point E/15 permet d'évaluer les effets et l'impact des dispositions d'atténuation et d'adaptation au changement climatique obtenus par la gestion de l'aire protégée. Le questionnaire propose d'évaluer les effets et les impacts d'atténuation et d'adaptation au changement climatique comme conséquence de la gestion de l'aire protégée, par rapport aux catégories des valeurs les plus importantes du contexte d'intervention aux 
points CTX 4 ; CTX 5 et CTX 7. II est possible de compléter les champs d'analyse avec des éléments spécifiques ou nécessaires à la gestion de l'aire protégée étudiée. Les dispositions d'atténuation et d'adaptation au changement climatique doivent être identifiées, puis le degré de réalisation actuel des effets et de l'impact de l'atténuation et de l'adaptation au changement climatique au profit de la conservation estimé en fonction de critères positifs ou négatifs selon les barèmes indiqués. Étant donné la complexité de la question, les évaluateurs internes sont invités à préciser les aspects les plus importants relatifs aux effets et à l'impact de l'action de l'aire protégée sur l'atténuation et l'adaptation au changement climatique, dans l'espace relatif aux observations.

\section{E/16}

Effets et impact

\section{Effets et impact sur les services écosystémiques}

Le point E/I6 essaye d'évaluer les effets et l'impact de la gestion de l'aire protégée sur le maintien et la valorisation des services écosystémiques. Le questionnaire propose d'évaluer les effets et les impacts sur le maintien et la valorisation des services écosystémiques, comme conséquences de la gestion de l'aire protégée. L'analyse s'appuie sur l'élaboration d'une liste rapportant tous les services écosystémiques les plus importants sur la base des éléments du contexte de l'intervention aux points CTX 7.1. Les services écosystémiques maintenus ou valorisés par la gestion de l'aire protégée doivent être identifiés, puis le degré actuel des effets et de l'impact de la gestion de l'aire protégée sur les services écosystémiques estimé en fonction de critères positifs ou négatifs selon les barèmes indiqués. Étant donné la complexité de la question, les évaluateurs internes sont invités à préciser les aspects les plus importants relatifs aux effets et à l'impact de l'action de l'aire protégée sur le maintien et la valorisation des services écosystémiques, dans l'espace dédié aux observations.

\subsection{Analyse des résultats}

\subsubsection{Analyser l'évaluation de l'efficacité de la gestion}

L'analyse des résultats permet de détecter les faiblesses de la gestion et les opportunités d'amélioration pour le futur. Comme de nombreux outils d'évaluation tels que l'Évaluation rapide et l'établissement des priorités de la gestion des AP (RAPPAM) ou l'Outil de suivi de la Banque mondiale (PAMETT), le Formulaire BIOPAMA utilise des notes simples.

L'évaluation des six éléments du Cadre de la CMAP de I'UICN (contexte de la gestion, planification, intrants, processus, résultats, effets et impacts) permet de savoir dans quelle mesure la gestion atteint

La plupart des évaluations de l'efficacité de la gestion se basent sur des informations recueillies antérieurement et comparant une ou plusieurs années d'exercice. Pour cette première année de campagne BIOPAMA de renforcement des capacités, les résultats fournis par le Formulaire serviront de niveau de base (baseline). L'analyse des résultats du Formulaire BIOPAMA permettra avant tout de bien comprendre la situation actuelle de gestion de l'aire protégée, sans comparaison par rapport à une année précédente.
: Revoir les FICHES 4 et 5 pour bien assimiler les aspects conceptuels de l'analyse de l'efficacité de gestion d'une aire protégée.

les cibles qu'elle s'est fixée et quelle est l'efficacité avec laquelle l'aire protégée maintient sa biodiversité et autres valeurs intrinsèques. Une partie importante de l'analyse est celle où l'on identifie dans quelle mesure les résultats sont dus aux interventions de la gestion ou à d'autres facteurs qui relèvent, ou pas, du gestionnaire concerné. II est possible qu'une aire protégée bien gérée voie encore disparaître sa biodiversité (par exemple, à cause du changement climatique) alors qu'inversement, des aires protégées gérées peu efficacement peuvent, dans certaines circonstances, conserver leurs valeurs. II est

: Les notes permettent de donner un aperçu instantané des efforts de conservation mais en même temps de simplifier les problématiques complexes de gestion des aires protégées. Néanmoins, il faudra utiliser la visualisation du Formulaire BIOPAMA pour aider à identifier des problématiques potentielles de gestion, solubles grâce à des analyses approfondies. Se limiter aux notes pour prendre des décisions peut conduire à de mauvaises interprétations et à choisir des solutions erronées dans la gestion d'une aire protégée. 
important de comprendre les causes des succès et des échecs de la gestion : sans cette analyse, toute tentative pour améliorer les performances serait inefficace.

\subsubsection{Protocoles d'analyse}

Une fois le Formulaire rempli intégralement, l'interprétation et l'analyse des résultats peuvent commencer. II est important de faire cette analyse en présence de toutes les personnes ressources liées à la gestion du parc.

Les étapes suivantes sont données pour guider le coach dans l'analyse :

- Faire un bref rappel des démarches de remplissage aboutissant au résultat final, avec le radar.

- Afficher la visualisation des résultats : les radars et les histogrammes.

- Afficher le niveau de collecte des informations sur l'état du contexte de l'intervention et l'état de l'efficacité de la gestion obtenus au cours du test et estimé et reporté ci-dessous. (Ex : PN 4, Pays 1 : état du contexte $=60 \%$; état de l'efficacité de la gestion $=80 \%$ ).

- Commencer par une analyse générale de la visualisation : y a-t-il des contradictions ? Quels sont les points concordants? Les points importants?

- Voir le tableau de compilation des informations.
II peut s'avérer nécessaire de revoir certaines rubriques et de changer les notations puisque en contradiction entre eaux.

- Entrer dans les détails. L'analyse suit le contexte d'intervention et les éléments du cycle de gestion. La section suivante vous permettra de mieux analyser les différents aspects des résultats obtenus.

: II n'est absolument pas professionnel de changer les évaluations pour améliorer les notes. Les modifications sont admises en cas d'erreur ou d'incohérence entre les valeurs. II s'agit d'appréciations qui pourraient se produire une fois terminé le remplissage d'une section ou du Formulaire en entier.

\subsubsection{Analyse du contexte de l'intervention}

L'analyse du contexte de l'intervention permet d'évaluer la prise en compte des valeurs importantes de l'aire protégée dans la gestion de l'aire protégée.

\subsubsection{Analyse de l'efficacité de la gestion}

\section{Contexte de la gestion, planification et intrants}

Le contexte de la gestion, la planification et les intrants sont liés puisque ces trois éléments fixent

\footnotetext{
Exercice 10 - Analyse Contrainte - Support - Pouvoir des acteurs 2

Dans l'AP 3, Pays 3, l'analyse des contraintes et supports extérieurs avait facilement révélé l'existence d'une forte aptitude négative d'une des quatre communautés participantes au comité de gestion. L'équipe de gestion de la réserve semblait ne pas donner trop d'importance à la problématique, étant donné la possibilité d'assurer la cogestion grâce à une majorité (trois communautés sur les quatre). L'interaction entre l'équipe de gestion de la réserve et les coaches a permis d'analyser plus en profondeur la problématique et les raisons de divergence, et de proposer des initiatives visant à une gouvernance de l'aire protégée plus équilibrée et de meilleure qualité.
}

\section{Questions}

1. Sans entrer dans une analyse détaillée, un coach aurait-il pu recevoir directement l'information de la part de l'équipe de gestion sur l'aptitude négative d'une des quatre communautés participantes au comité de gestion?

2. Pourquoi l'analyse des contraintes et appuis qui peuvent influencer la décision de gestion et de gouvernance d'une aire protégée doit-elle être aussi analysée par rapport au pouvoir des acteurs ?

3. Considérez-vous que même dans le cas d'une gouvernance par le gouvernement, l'analyse " Contrainte - Support - Pouvoir des acteurs » est nécessaire ?

4. Vous êtes en faveur d'une amélioration de la gouvernance : dans ce cas, seriez-vous en mesure de préciser les autres éléments d'analyse du Formulaire à ce sujet ?

5. Estimez-vous que la qualité de la gouvernance puisse aussi être identifiée comme un objectif et un impact recherchés, au même titre que la sauvegarde d'une population animale ou d'habitats ? 
les aspects de l'intervention directe qui sont ensuite mis en œuvre par le processus.

\section{Contexte de la gestion}

Le contexte de la gestion est déterminé sur la base des éléments du contexte de l'intervention.

Le contexte de l'intervention correspond à l'ensemble des états ou conditions dans lesquels s'inscrit la conservation de l'aire protégée.

Le contexte de la gestion fixe comme sujets clés de gestion par l'aire protégée un certain nombre d'éléments du contexte de l'intervention.

Selon cette distinction, le contexte de l'intervention identifie plusieurs sujets importants de gestion (aspects de gouvernance, dispositions de statut de classement, espèces animales ou végétales, habitats, changements dans la couverture des sols, atténuation et adaptation au changement climatique, services écosystémiques) et le contexte de la gestion choisit les éléments sur lesquels focaliser les interventions de gestion et de gouvernance de l'aire protégée. Les principaux sujets de gestion doivent absolument constituer la base des indicateurs pour le suivi et l'évaluation des efforts de conservation.

La détermination des sujets clés de gestion passe généralement par la démarche suivante :

- Analyse des indications générales et des sujets clés de gestion, fournis par le contexte de l'intervention ;

- $\quad$ Prise en compte des sujets clés dans la gestion, qui devront être ensuite utilisés pour les analyses des éléments du cycle de gestion des aires protégées.

Les sujets clés de gestion constituent les valeurs et les éléments importants de l'aire protégée. L'analyse du contexte de la gestion prend également en compte les

\section{Exercice 11 - Analyse du contexte de la gestion}

L'exercice vise à analyser et utiliser les résultats des analyses du contexte de l'intervention pour définir les éléments à insérer dans le contexte de la gestion, et à établir les liens avec les autres éléments du cycle de gestion d'une aire protégée. Le Formulaire accompagne la démarche d'analyse des efforts de gestion sur la base des choix effectués dans le cas du contexte de la gestion. Le choix des éléments du contexte de la gestion apparait pourtant fondamental puisqu'il déterminera la validité de l'analyse.

L'analyse du contexte de l'intervention dans le Parc National 4, Pays 1 avait relevé que les éléments de conservation les plus importants étaient, pour la plupart, correctement listés dans le document de planification, mais que leur priorisation en terme de gestion n'était pas précisée. À l'aide de la liste des espèces potentiellement présentes dans l'aire protégée, associée avec les indications de la Liste rouge de l'UICN disponible sur le site DOPA Explorer, l'exercice avait permis d'approfondir les priorités d'intervention pour les sujets de conservation. Ensuite, l'exercice de priorisation d'intervention avait identifié les différents efforts de conservation pour les valeurs et les aspects importants de l'aire protégée. L'analyse avait mis en évidence d'importants sujets de conservation avec peu ou aucun effort de conservation par rapport à d'autres valeurs prioritaires. L'analyse était rendue significative par l'absence d'objectifs de gestion (voir exercice 4).

Pour le bon déroulement de cet exercice, il est important de préciser que le PN 4, Pays 1 est soumise à de fortes contraintes d'accessibilité et de disponibilité des ressources.

\section{Questions}

1. Pensez-vous qu'une analyse approfondie pourrait se faire sans le support d'instruments tels que le Formulaire, le DOPA Explorer, la Liste rouge de l'UICN, etc. ?

2. Pensez-vous que le personnel de l'aire protégée, une des plus riches zones en biodiversité d'Afrique, avait concentré son attention sur un nombre très réduit de sujets de conservation en raison de l'insuffisance des ressources, d'informations, de suivi-évaluation, de bonne planification,...

3. Quels sont les autres éléments à prendre en compte dans le contexte de la gestion ?

4. Quels sont les critères pour définir un élément du contexte de l'intervention comme élément du contexte de la gestion, à analyser dans le cycle de gestion d'une aire protégée ? 


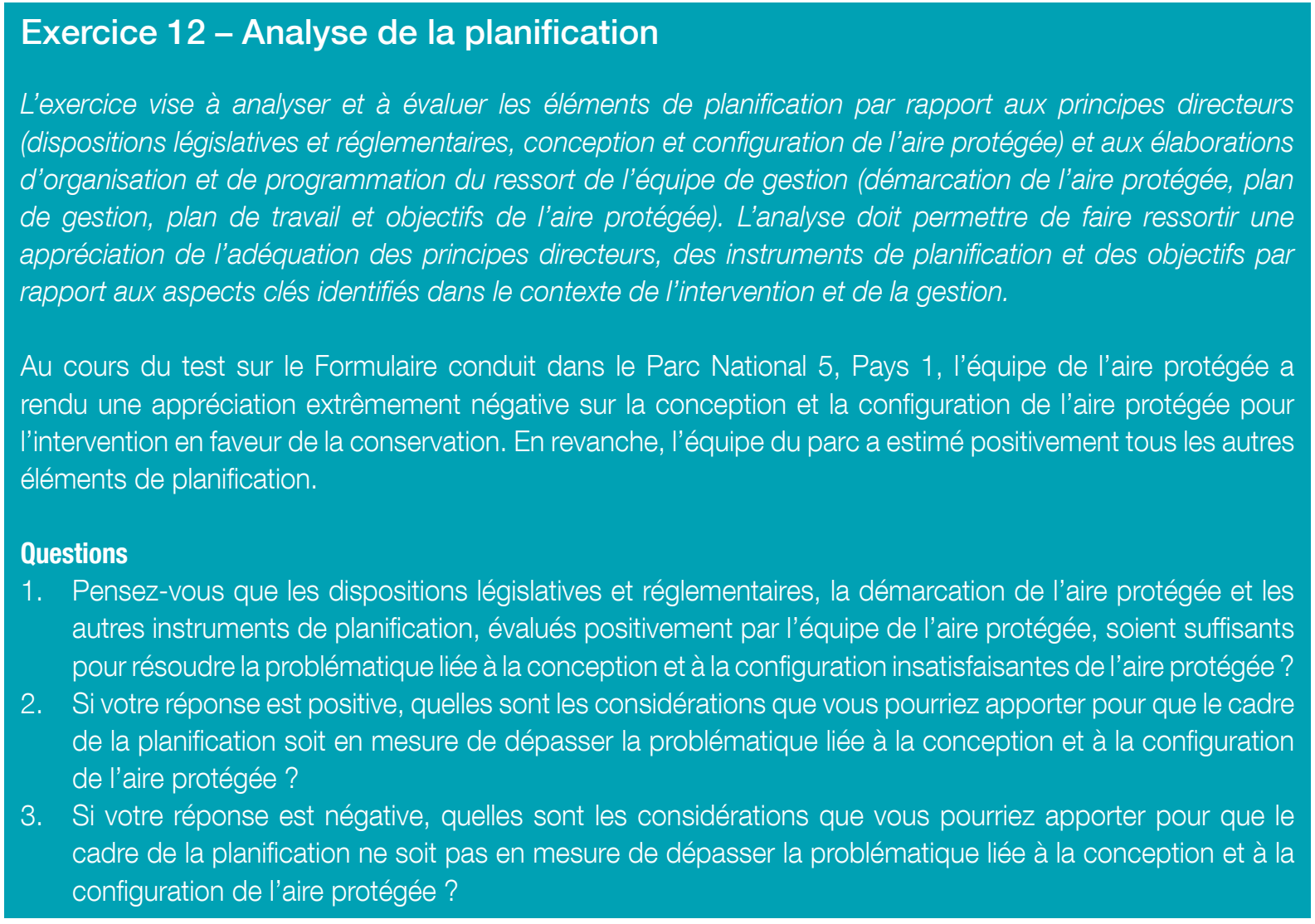

contraintes ou soutiens par l'environnement politique et civil externe, et les menaces (pressions, menaces et vulnérabilités) qui pèsent sur l'aire protégée suite à l'analyse effectuée dans le cadre du contexte d'intervention afin de constituer un cadre complet de la situation de l'intervention.

Les résultats de l'analyse des différents sujets permettent de déterminer l'état du contexte de la gestion par rapport à l'analyse plus large du contexte de l'intervention.

\section{Planification}

Sur la base des considérations tirées du contexte de l'intervention et de la gestion, les éléments de la planification analysent les sujets guides à respecter dans les dispositions de programmation (dispositions législatives et réglementaires, conception et configuration de l'aire protégée) et les dispositions spécifiques d'organisation des interventions définies par l'équipe de gestion (démarcation de l'aire protégée, plan de gestion, plan de travail et objectifs de l'aire protégée).

Les résultats de l'analyse des sujets de planification permettent de vérifier l'adéquation des dispositions guides (par ex. les dispositions législatives et réglementaires et la conception de l'aire protégée peuvent favoriser ou non la gestion et la gouvernance de l'aire protégée) et la prise en compte des sujets clés par les instruments de planification (par ex. le plan de gestion et le plan de travail annuel peuvent avoir identifié d'autres valeurs et éléments importants par rapport à l'analyse effectuée à l'aide du Formulaire, en raison de l'évolution du contexte, ou plus simplement d'une analyse insuffisante).

\section{Intrants}

Les sujets d'analyse de l'élément de gestion "Intrants » concernent les ressources diverses dont l'aire protégée dispose pour assurer les interventions et les objectifs définis par les éléments de planification.

Les éléments relatifs aux intrants permettent d'évaluer l'adéquation entre les interventions et les objectifs définis par la planification, et les ressources dont dispose l'aire protégée (informations de base, ressources humaines, financières et infrastructures, équipements et installations).

\section{Analyse du processus de gestion}

Le processus de gestion représente certainement l'élément le plus important du cycle de gestion. Les précédents éléments : le contexte de la gestion, la planification et les intrants permettent d'introduire les différents aspects de l'intervention directe qui sont introduits dans le processus de gestion. Les éléments suivants - résultat (output) et effets/impact 


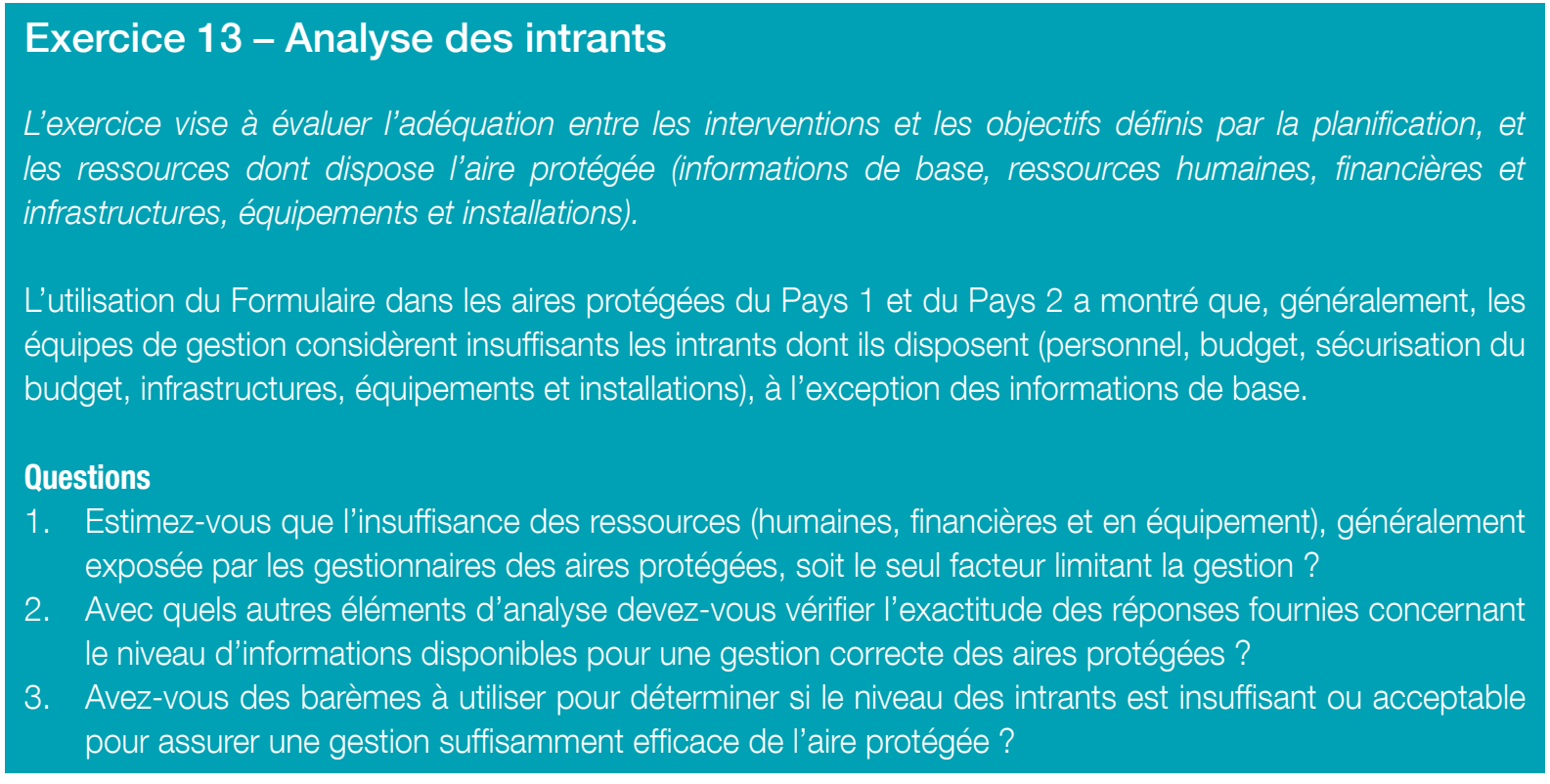

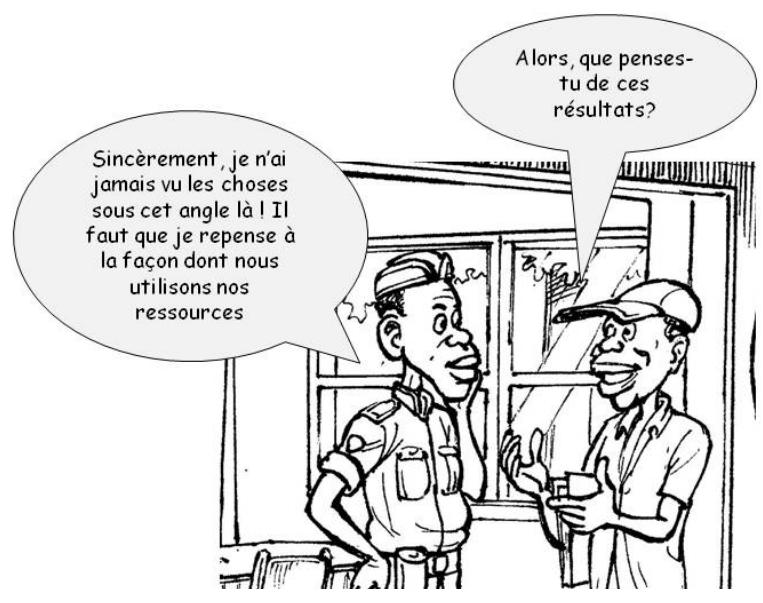

(outcome) - fournissent la valeur des produits et l'impact de l'action du processus de gestion.

En raison de l'importance de cet élément dans le cycle de gestion, le Formulaire présente plusieurs éléments d'analyse correspondant en majorité aux indications de l'étude Global Study 2010 (Hockings et al, 2008). Les sujets du processus sont classés en six groupes :

- Systèmes et processus de gestion interne ;

- Protection/gestion ;

- Relations avec les parties prenantes ;

- Gestion du tourisme ;

- Suivi et recherche ;

- Gestion des effets du changement climatique et des services écosystémiques.

Le premier groupe (systèmes et processus de gestion interne) concerne l'organisation des activités de gestion, les autres concernent les interventions diverses au profit de la conservation et de la valorisation des ressources naturelles et de la biodiversité.
Les résultats de l'analyse des sujets du processus de gestion permettent d'évaluer l'organisation et le déroulement des interventions de conservation des sujets identifiés par le contexte, programmés par la planification, mis en œuvre avec les moyens précisés dans les intrants, et qui enfin doivent produire les résultats et les effets/impacts programmés et espérés.

\section{Systèmes et processus de gestion interne :}

Ce groupe permet d'analyser six aspects : 1) les capacités et le niveau de formation du personnel ; 2) les politiques et les procédures de gestion des ressources humaines ; 3 ) les systèmes et les processus de gestion des ressources humaines ; 4) l'administration et le leadership interne ; 5) la gestion administrative-comptable et financière ; et 6) l'entretien des infrastructures, des équipements et des installations.

\section{Protection et gestion}

Cette section vise à analyser l'existence et l'efficacité des mesures adoptées au profit de la protection et de la gestion active en faveur du maintien des espèces, des habitats, des processus écologiques, de la gestion durable des ressources naturelles, de la réduction des menaces, de l'atténuation et de l'adaptation aux effets du changement climatique, du maintien des services écosystémiques, etc. À ce propos, quatre aspects sont analysés : 1) la gestion des valeurs et des aspects importants de l'aire protégée ; 2) les systèmes de protection des valeurs et des aspects importants de l'aire protégée ; 3) le contrôle de l'aire protégée ; et 4) l'application des lois. 


\section{Exercice 14 - Analyse des systèmes et processus de gestion}

L'utilisation du Formulaire dans les aires protégées dans le Pays 1 et le Pays 2 a montré que, généralement, les équipes de gestion considèrent positivement le système et le processus de gestion interne. Cette appréciation n'est pas souvent confortée par les résultats et les effets produits en termes de conservation. Les résultats affichés peuvent être interprétés de plusieurs manières :

- Difficulté à l'autoévaluation du personnel de gestion des aires protégées ;

- Idée que les systèmes et les processus de gestion interne répondent à des objectifs spécifiques et séparés des objectifs globaux de conservation ;

- Absence d'une liaison action - résultat - effet - impact - efficacité de la gestion ;

- Estimations favorables pour maintenir de bonnes relations interpersonnelles du staff de gestion des aires protégées ;

- Potentiel existant de gestion des aires protégées qui ne manque que de moyens (intrants) pour produire plus de résultats et les effets attendus ;

- Insuffisances du Formulaire pour l'analyse des systèmes et des processus de gestion interne ;

- Autres.

Questions

1. D'après vous, quelles considérations justifient l'évaluation générale positive du système et du processus de gestion interne par les équipes des aires protégées ?

2. Que pourriez-vous suggérer au personnel de gestion des aires protégées pour analyser le plus objectivement possible leur système et le processus de gestion interne?

3. Comme pourriez-vous essayer de mieux évaluer et ensuite mieux orienter les systèmes et les processus de gestion interne vers l'obtention de résultats et d'impacts de conservation ?

\section{Relations avec les parties prenantes}

Cette rubrique essaie d'estimer le degré d'implication des différents acteurs dans la gestion du contexte de l'intervention et de l'aire protégée (le cas échéant), l'adéquation et les effets des interventions visant à obtenir des avantages pour les collectivités riveraines y compris des programmes d'éducation environnementale (connaissance de la nature) et d'éducation à l'environnement (aptitude à une gestion durable des ressources naturelles). Les sujets sont analysés selon trois aspects : 1) l'implication des communautés locales, des ayants droit et des parties prenantes ; 2) les avantages/ assistance appropriés au profit des collectivités ; et 3) les relations avec les acteurs.

\section{Gestion du tourisme}

Cette section doit permettre d'évaluer l'adéquation des installations et des services au profit des visiteurs (tourisme et éducation environnementale) et les dispositions de prise en charge et d'atténuation des impacts des activités touristiques, appropriées pour

\section{Exercice 15 - Analyse de la protection}

Les phases test du Formulaire dans les aires protégées dans le Pays 1 et Pays 2 ont montré que, généralement, les équipes de gestion accordent des estimations positives (valeurs de 60-70 \%) pour le contrôle du territoire et d'application de la loi, mais des valeurs inférieures pour la protection et la gestion spécifique des sujets et des éléments importants pour la conservation.

\section{Questions}

1. D'après vous, pourquoi les équipes de gestions attribuent-elles une évaluation positive au système de contrôle du territoire et d'application de la loi, et des valeurs inférieures pour la protection et la gestion spécifiques des sujets de conservation ?

2. Considérez-vous que le contrôle du territoire soit une mesure suffisante pour assurer la sauvegarde du patrimoine en biodiversité d'une aire protégée ?

3. Pourquoi les équipes de gestion semblent-elles plus orientées vers un contrôle traditionnel et territorial que vers des cibles de conservation spécifique (habitats, espèces, etc.) ?

4. Enfin, considérez-vous que l'estimation de contrôle sur environ $3 / 4$ de la surface d'une aire protégée est une valeur : 1) insuffisante ; 2) suffisante ; 3) bonne ou 4) excellente ? 


\section{Exercice 16 - Analyse des relations avec les parties prenantes}

Les résultats de l'utilisation du Formulaire dans les aires protégées dans le Pays 1 et Pays 2 ont montré que, généralement, les équipes de gestion estiment positivement l'implication des acteurs, mais les résultats en termes d'avantages pour les riverains sont assez faibles. De plus, les résultats des tests de terrain montrent que les actions d'éducation environnementale, qui auraient pu contribuer à la réduction des pressions et menaces à court terme, sont insuffisantes.

\section{Questions}

1. Etes-vous certain de pouvoir faire comprendre aux gestionnaires des aires protégées la distinction entre : 1) implication dans les décisions ; et 2) communication des interventions et entre : a) implication dans la gestion d'une aire protégée ; et b) communication sur les dispositions de gestion d'une aire protégée ?

2. Pourquoi les avantages en faveur des riverains sont-ils aussi faibles, malgré les valeurs positives d'implication dans la prise de décision des ayants droit ?

3. Que suggérez-vous d'initier pour une réduction des conflits parc-populations, entre : 1) des programmes d'éducation environnementale (connaissance de la nature) ; et 2) des actions liées à l'éducation à l'environnement (aptitude à une gestion durable des ressources naturelles)

assurer le maintien des valeurs et de l'importance de l'aire protégée. L'analyse est organisée en deux points : 1) la gestion des visiteurs ; et 2) l'impact des activités de tourisme.

\section{Suivi et recherche}

L'analyse évalue l'état, l'adéquation et les effets des activités de suivi, de recherche et de biosuivi en appui à la gestion des valeurs et des aspects importants de l'aire protégée par rapport aux efforts de gestion et à l'ampleur et à la gravité des menaces. L'évaluation prévoit deux aspects : 1) les systèmes de suivi des valeurs et des aspects importants de l'aire protégée ; et 2) la recherche et le biosuivi.

\section{Gestion des effets du changement climatique et des services écosystémiques}

L'analyse doit permettre d'évaluer les dispositions prises par l'aire protégée pour atténuer et adapter les effets du changement climatique, en faveur de la conservation et de la valorisation des services écosystémiques rendus pour le bien-être humain. L'évaluation concerne deux aspects : 1) la gestion des effets du changement climatique ; et 2) les services écosystémiques rendus.

\section{Exercice 17 - Analyse de la gestion du tourisme}

L'utilisation du Formulaire dans les aires protégées dans le Pays 1 et le Pays 2 pour les aspects liés à la valorisation de la biodiversité par le tourisme a montré une large gamme de situations : a) aucune activité touristique ni visite d'éducation environnementale ; b) des activités touristiques en gestion directe ; c) des actions d'écotourisme attribuées à des agences privées. À l'exception d'une aire protégée, qui affiche une forte organisation du secteur du tourisme en raison de l'exceptionnalité de son patrimoine, les autres sites de conservation présentent une faible organisation des actions d'écotourisme et d'éducation environnementale, et en conséquence une encore plus faible activité de gestion de l'impact du tourisme.

\section{Questions}

1. Avant ces points d'analyse, disposiez-vous des indicateurs concernant la valeur ou le potentiel touristique et, dans ce cas, par quels autres éléments du Formulaire ?

2. Dans le cas où la gestion touristique est attribuée à un opérateur privé, les points d'analyse sont-ils à évaluer : 1) avec l'équipe du parc ; 2) avec l'opérateur touristique ; 3) avec l'équipe du parc et l'opérateur touristique?

3. En présence de faibles ou très faibles actions touristiques et d'éducation environnementale, estimezvous qu'il est préférable : 1) de ne pas évaluer ce secteur d'intervention ; ou bien 2) de l'analyser malgré l'activité de faible importance dans la gestion générale de l'aire protégée ? 


\section{Exercice 18 - Analyse du suivi et de la recherche}

L'auto-évaluation de la gestion des aires protégées au cours de la phase test du Formulaire, dans le Pays 1 et au Pays 2, a toujours affiché des estimations positives pour le suivi, et très faibles, voire inexistantes, pour la recherche et le biosuivi (biomonitoring). Or des activités de recherche réduites ou inexistantes dans les aires protégées est compréhensible et acceptable en raison de la nature des interventions très spécialisées et couteuses. En revanche, l'estimation positive des actions de suivi ne correspond souvent pas à la réalité puisque les estimations des valeurs de la biodiversité, en termes par ex. de tendance et de répartition, sont généralement insuffisantes ou le niveau de fiabilité de l'information est moyen ou faible.

\section{Questions}

1. Pourquoi les équipes des aires protégées considèrent-elles que leur système de suivi est moyennement valable, alors qu'elles ne disposent pas des informations et d'un suffisant niveau de fiabilité des données à afficher et pour assurer la bonne gestion des valeurs et des éléments importants ?

2. Estimez-vous que, en raison des moyens mis à la disposition des équipes de gestion, l'information mise à la disposition des responsables des aires protégées soit suffisante pour assurer une gestion correcte de l'aire protégée?

3. La recherche ou le biosuivi sont-ils vraiment indispensables pour la gestion d'une aire protégée et méritentt-ils d'être inclus dans les systèmes d'évaluation?

\section{Analyse des résultats et effets/impacts}

Les éléments du cycle de gestion qui analysent les résultats (Outputs) et les effets/impacts (Outcomes) sont associés puisque les deux conjointement permettent d'analyser les réalisations des efforts de conservation d'une aire protégée. L'analyse de ces deux éléments contribue à évaluer la chaine Action Résultat - Effet - Impact - Efficacité de la gestion décrite dans l'exercice 5.

\section{Résultats (Outputs)}

L'analyse se propose d'évaluer la mise en œuvre du plan de travail et d'estimer dans quelle mesure l'aire protégée a atteint les principaux résultats de la programmation annuelle ou pluriannuelle des efforts de conservation. Pour y arriver, l'exercice prévoit deux aspects : 1) le degré de mise en œuvre des principales activités du programme de travail annuel (R1) ; et 2) le niveau de réalisation des principaux résultats attendus du plan de

\section{Exercice 19 - Analyse de la gestion des effets du changement climatique et des services écosystémiques}

Les tests du Formulaire ont permis d'introduire ou de mieux préciser, dans presque toutes les aires protégées visitées, les éléments de base et des exemples de possibles interventions à entreprendre en faveur de l'atténuation et de l'adaptation des effets du changement climatique, en faveur de la conservation et de la valorisation des services écosystémiques. Les résultats obtenus permettent de considérer que ces mesures sont faibles ou inexistantes en raison aussi de l'absence d'informations, de la perception des phénomènes ou d'un impact évident par l'équipe de gestion. Toutefois, la schématisation des services écosystémiques à l'aide d'un tableau récapitulatif permet aux équipes de l'aire protégée d'identifier, d'afficher et d'estimer leurs interventions et éventuellement de programmer des actions à l'avenir en faveur des services rendus pour le bien-être humain.

\section{Questions}

1. Estimez-vous que le Formulaire, en introduisant des aspects connus de gestion, mais pas encore standardisés (par ex. changement climatique et services écosystémiques), dans le système de gestion d'une aire protégée, puisse apporter un renforcement des capacités et indirectement l'amélioration de la gestion d'un site de conservation?

2. L'attention insuffisante portée aux effets du changement climatique doit être interprétée comme : 1) le résultat d'une information vague sur le sujet ; 2) un manque de ressources pour entreprendre des actions ; ou 3) l'absence d'une approche proactive par l'équipe de gestion ?

3. Les services écosystémiques doivent-ils être mieux valorisés et que proposez-vous pour améliorer l'affichage de ces importants résultats et effets de l'aire protégée ? 
travail annuel ou pluriannuel (R2). L'exercice vise à analyser la mise en œuvre et les produits fournis par les interventions de gestion. L'articulation de la première question (R1) doit permettre d'estimer le niveau de mise en œuvre des actions qui contribuent à générer des résultats. La deuxième question (R2) de manière conjointe doit permettre d'évaluer les résultats que l'aire protégée a atteints en agissant dans un but défini à travers les actions.
En général, la difficulté pour obtenir des réponses sur ce sujet dépend de la logique et de l'organisation des instruments de planification. II est pourtant important de donner le maximum d'attention à cette phase de l'analyse de l'évaluation de l'efficacité de la gestion d'une aire protégée.

\section{Effets/impacts (Outcomes)}

L'analyse se propose d'estimer dans quelle mesure les produits du processus de gestion

\section{Exercice 20 - Analyse des résultats}

Les phases tests dans les Pays 1, Pays 2 et Pays 3 montrent que l'organisation des interventions des aires protégées ne répond pas pleinement au processus Action - Résultat - Effet - Impact - Efficacité de la gestion. Plusieurs cas de figure apparaissent dans le montage du plan de travail :

- II est organisé seulement par actions ;

- II ne détermine pas les résultats des actions annuelles et encore moins ceux des actions pluriannuelles ;

- II ne fait pas de distinction entre actions et résultats ;

- II diffère des indications du Plan de gestion.

En conclusion, l'évaluation de l'efficacité de la gestion est rendue très difficile par l'absence ou le non-respect d'une logique d'intervention Action - Résultat - Effet - Impact divergent. Le résultat final est que souvent, le coach doit constater :

- L'absence d'une séparation claire et nette entre la mise en œuvre des actions et la réalisation des résultats ;

- L'absence de liaison fonctionnelle entre le plan de gestion et le plan de travail annuel.

Ci-dessous, trois cas de résultats de produits fournis par les interventions de conservation sont présentés pour analyse.

Exemple 1

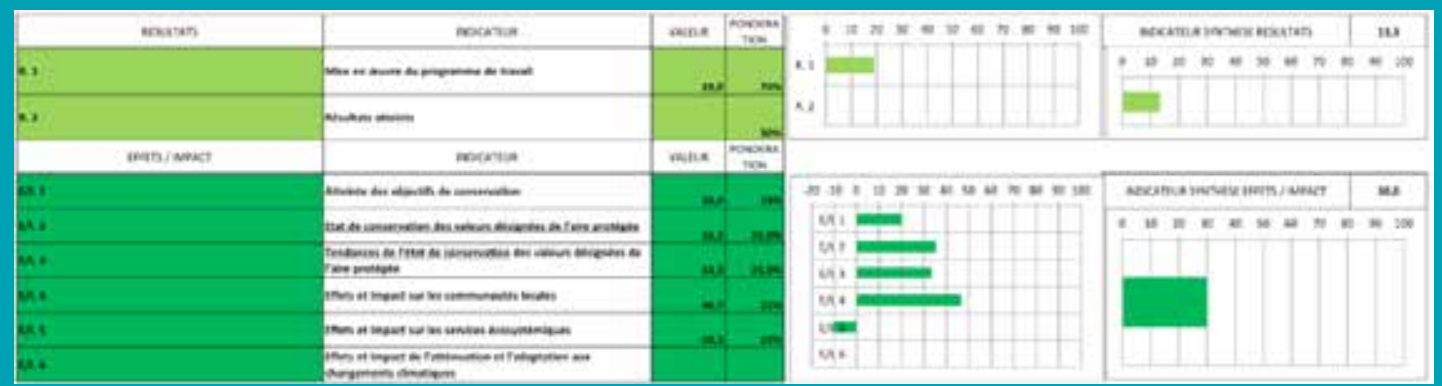

Exemple 2

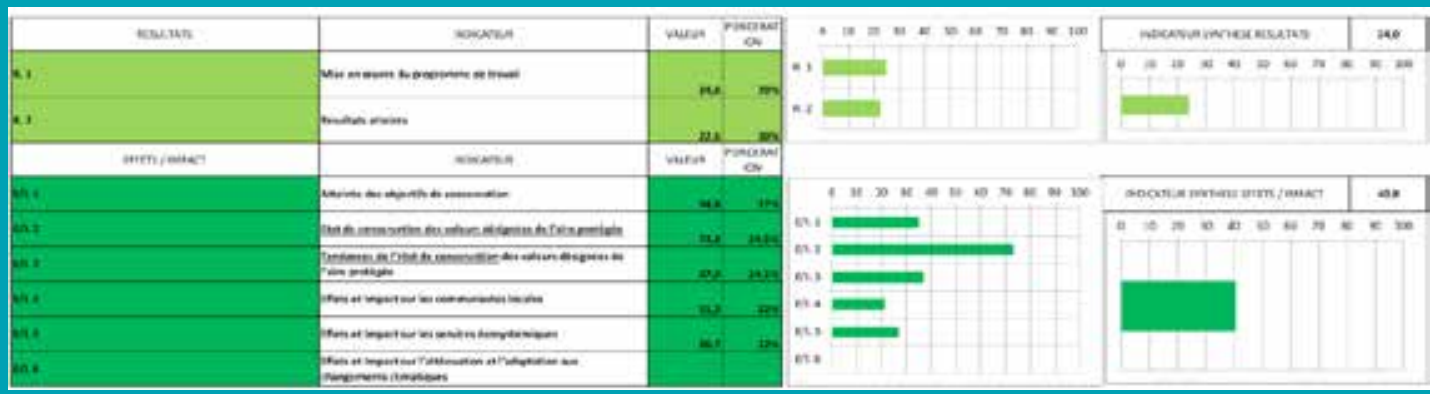




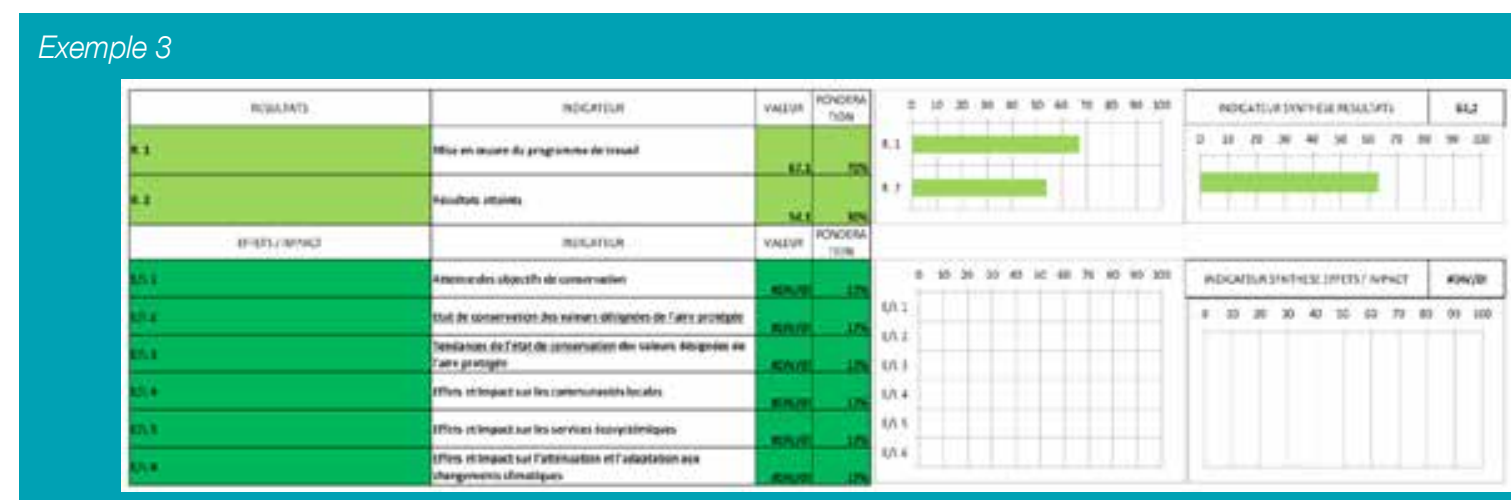

Questions

Avant de répondre, analysez bien les trois exemples tirés des tests de terrain et formulez vos analyses sur les résultats affichés.

Analyse générale

1. À votre avis, quel exemple correspond à une analyse apparemment complète et satisfaisante des résultats (outputs) : exemple 1 ; exemple 2 ; exemple 3 ?

2. Pourriez-vous repérer les éléments apparemment inexacts qui sont présents dans les trois exemples ? Faites-le pour chaque exemple.

Analyse spécifique :

3. Comment jugez-vous le fait que les exemples 1 et 3 affichent tous les deux un ou plusieurs éléments hors de la logique Action - Résultat - Effet - Impact ? S'agit-il d'erreurs commises au cours de l'évaluation ? Est-il possible que les résultats affichés soient corrects et dans ce cas quel aspect constitue toutefois une faiblesse dans l'organisation des instruments de planification, qu'il faudra noter dans les observations de l'analyse et éventuellement corriger en accord avec l'équipe de gestion de l'aire protégée ?

4. Comment jugez-vous le fait que l'exemple 2 montre des valeurs pour les résultats plus faibles que celles de l'élément d'évaluation effets/impacts ? S'agit-il d'erreurs commises au cours de l'évaluation ? Est-il possible que les résultats affichés soient corrects et, dans ce cas, quel aspect constitue toutefois une faiblesse dans l'organisation des instruments de planification, qu'il faudra noter dans les observations de l'analyse et éventuellement corriger en accord avec l'équipe de gestion de l'aire protégée ?

5. À quel niveau de la réflexion l'existence ou l'élaboration de valeurs de référence (benchmarks) pourrait-elle vous aider dans l'analyse?

de l'aire protégée ont eu des effets/impacts directs et ont contribué à réaliser les principaux objectifs de conservation ou de gouvernance. L'évaluation est complexe et pour cela six aspects d'analyse sont prévus : 1) réalisation des objectifs de conservation ; 2) état de conservation des valeurs désignées de l'aire protégée ; 3) tendances de l'état de conservation des valeurs désignées de l'aire protégée ; 4) effets et impacts sur les communautés locales ; 5) effets et impacts de l'atténuation et de l'adaptation au changement climatique ; 6) effets et impacts sur les services écosystémiques.
En général, la difficulté pour obtenir des réponses sur ce sujet dépend de l'application des principes de définition des objectifs par la chaine :

Ligne de base de conservation (baseline) - Conditions souhaitées de conservation; soutenus par la définition des valeurs des différents aspects:

Ligne de base (baseline) - Valeurs de référence (benchmarks) - Indicateurs - Objectif. 


\section{Exercice 21 - Analyse des effets et impacts (outcomes)}

Les phases tests dans les Pays 1, Pays 2 et Pays 3 montrent que l'orientation des interventions dans les aires protégées ne répond pas pleinement au processus de définition d'un objectif ou d'une cible de conservation. Selon ce principe théorique, il est nécessaire de définir une ligne de base de conservation au point « zéro » (baseline) pour définir les conditions de conservation souhaitées. En disposant d'une ligne de base et de cibles de conservation, il est possible de fixer l'objectif ou les objectifs de gestion. II apparait évident aussi que les indicateurs et les valeurs de référence, éléments en support de l'évaluation des effets et impacts entre la ligne de base et les conditions de conservation souhaitées, sont difficiles à trouver dans le document de planification. En conclusion, et à l'exception de quelques exemples positifs, l'estimation des effets et des impacts des interventions de conservation s'avère difficile, voire très difficile.

Plusieurs cas de figure concernant les objectifs des documents de planification et de gestion des aires protégées apparaissent :

- II n'y a pas d'objectifs ;

- Les objectifs sont très généraux, sans valeurs ciblées à atteindre ;

- Les objectifs précisent la cible de conservation, mais en l'absence de définition d'une ligne de base, il est complexe d'estimer les effets et l'impact attendus de l'intervention ;

- Il y a trop d'objectifs et en conséquence les interventions ne sont pas priorisées (par ex. sur la base des menaces) ;

- Les objectifs du plan de gestion sont ajustés, voire modifiés, dans les plans de travail ;

- $\quad .$.

En conclusion, l'évaluation de l'efficacité de la gestion est rendue très difficile par l'absence ou le non-respect de la logique d'intervention Ligne de base - Valeurs de référence - Indicateurs Objectif (Conditions souhaitées de conservation). Le résultat final est que, souvent, le coach doit constater :

- L'absence de repères pour estimer les effets et l'impact, parfois simplement la tendance générale positive ou négative de l'intervention, dans les différents domaines de la conservation ou de la gouvernance ;

- L L'impossibilité d'interagir fonctionnellement avec l'équipe de gestion des aires protégées pour effectuer des analyses approfondies et ciblées sur l'amélioration de l'efficacité de la gestion, avec obligation de limiter les conclusions à de simples recommandations générales.

Ci-dessous, trois cas de résultats de produits fournis par les interventions de conservation sont présentés pour analyse.

Exemple 4

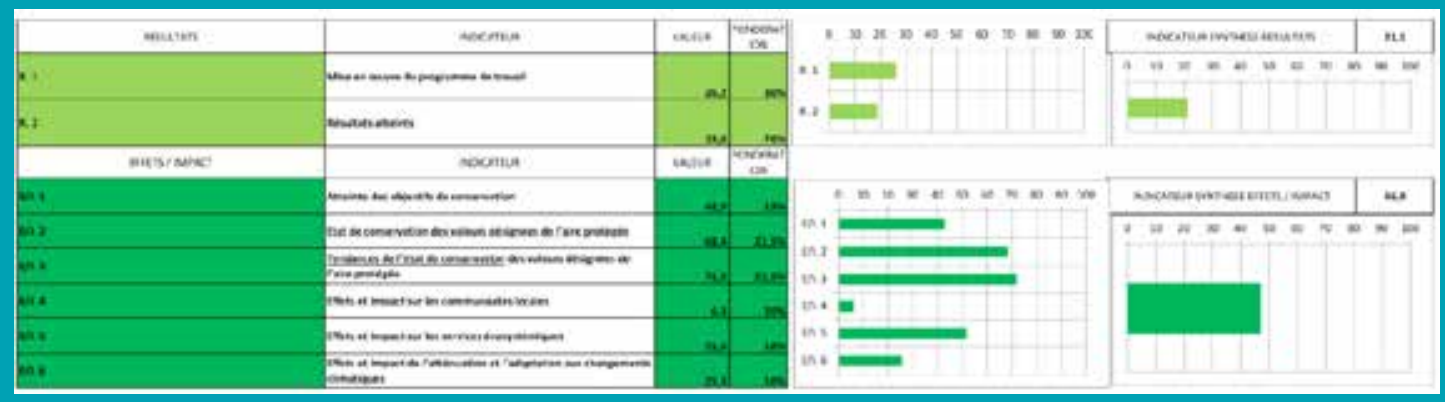


Exemple 5

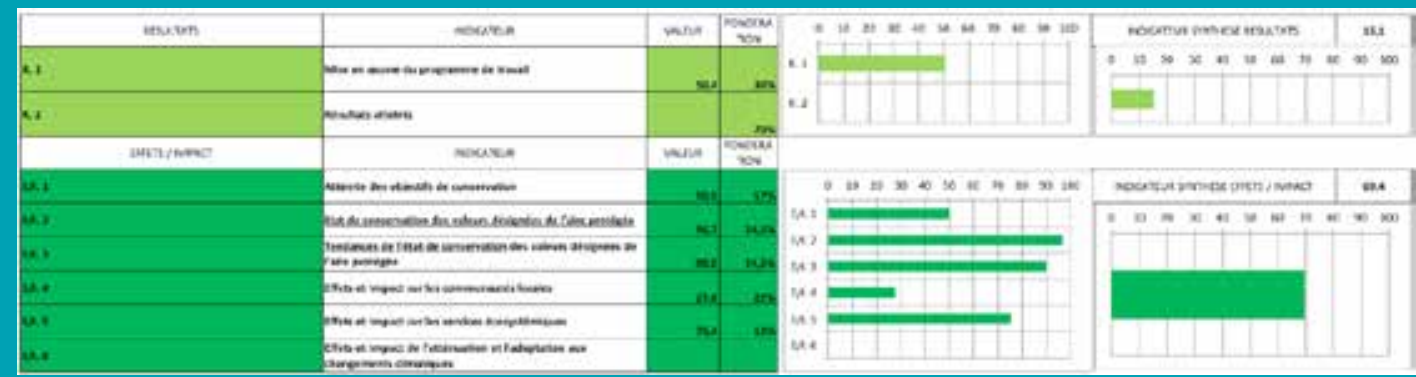

Exemple 6

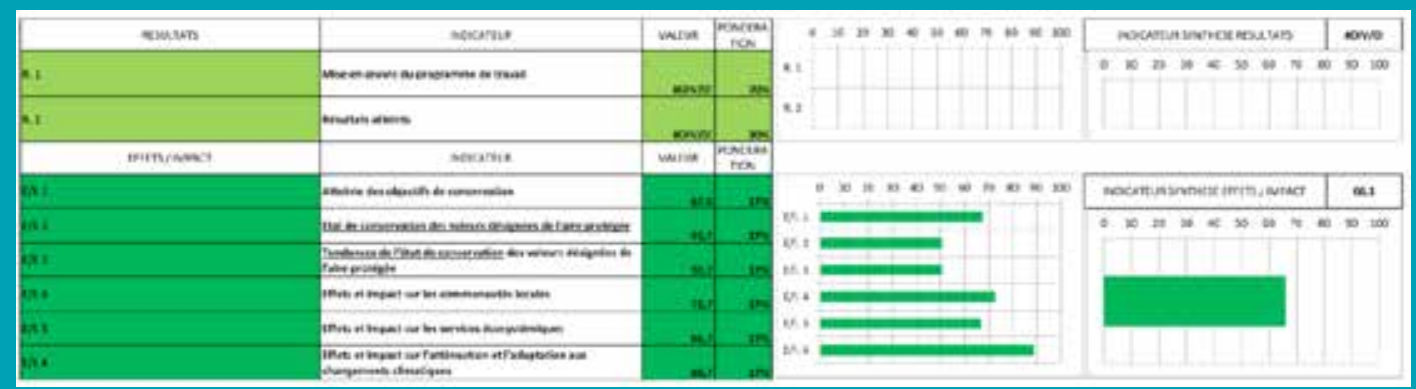

\section{Questions}

Avant de répondre, analysez bien les trois exemples tirés des tests de terrain et formulez vos analyses sur les estimations des effets et des impacts affichés.

Analyse générale

1. À votre avis, quel exemple, correspond à une analyse apparemment satisfaisante des effets/impacts (Outcomes) : exemple 4) ; exemple 5 ; exemple 6 ?

2. Quelles sont les différences entre les exemples 4 et 5 et 6 ?

3. Pourriez-vous repérer les éléments apparemment présents dans les trois exemples ?

4. Serait-il possible de déterminer une relation directe entre les objectifs du plan de gestion et les résultats à atteindre par les plans de travail annuels ?

5. Quelle valeur importante n'est pas mentionnée dans les exemples 4, 5 et 6 ?

Analyse spécifique

6. Comme jugez-vous le fait que l'exemple 4 affiche de faibles valeurs de mise en œuvre des actions et de réalisation des résultats, et que la valeur générale des effets/impacts soit en voie de devenir satisfaisante ?

S'agit-il d'erreurs commises au cours de l'évaluation ?

Est-il possible que les valeurs affichées et relatives aux effets/impacts soient correctes ? Et dans ce cas, quels aspects de cette évaluation faudra-t-il approfondir et éventuellement améliorer dans leur définition, en accord avec l'équipe de gestion de l'aire protégée?

7. Comme jugez-vous le fait que l'exemple 5 affiche seulement des valeurs de mise en œuvre des actions, aucun résultat atteints et malgré cela les estimations des effets/impacts sont bien définis pour tous les sujets d'analyse?

S'agit-il d'erreurs commises au cours de l'évaluation ?

Est-il possible que les valeurs affichées et relatives aux effets/impacts soient correctes et, dans ce cas, quels aspects de cette évaluation faudra-t-il approfondir et éventuellement améliorer dans leur définition, en accord avec l'équipe de gestion de l'aire protégée ?

8. Comment jugez-vous le fait que l'exemple 6 affiche seulement les estimations des Effets/Impacts ? S'agit-il d'erreurs commises au cours de l'évaluation ?

Existe-t-il la possibilité que les valeurs affichées et relatives aux effets/impacts soient correctes et, dans ce cas, quels aspects de cette évaluation faudra-t-il approfondir et éventuellement améliorer dans leur définition, en accord avec l'équipe de gestion de l'aire protégée ? 


\subsection{Formulation des propositions d'amélioration}

Le Formulaire constitue un support à la collecte et à l'organisation des informations sur le contexte de l'intervention et l'efficacité de la gestion des aires protégées. Une fois que l'outil affiche les résultats, ces données doivent être analysées pour faire comprendre à l'ensemble de l'équipe de gestion de l'aire protégée les points positifs et négatifs des interventions de la conservation, tirer des conclusions et formuler des propositions d'amélioration. À la fin de chacun des six éléments de la gestion des aires protégées, un tableau aide à faire un résumé de la situation et, dans ce cas précis, à fixer des objectifs ou les états de conservation souhaités. Les objectifs rapportés dans ce tableau constituent déjà des propositions d'amélioration pour la gestion de l'aire protégée.

Ce processus d'analyse et de recherche de solutions peut être supporté par des instruments de résolution des problèmes et de prise de décision (problem solving et decision making). Cidessous, quelques principes de ces instruments puis des études de cas issues des phases tests.

\subsubsection{Résolution des problèmes et prise de décision}

La résolution des problèmes et la prise de décision sont des compétences importantes pour les gestionnaires d'aire protégée. La résolution des problèmes implique souvent la prise de décision, et la prise de décision est particulièrement importante pour la gestion et le leadership. La résolution des problèmes et la prise de décision sont étroitement liées, et chacune exige de la créativité dans l'identification et l'élaboration de solutions. II est extrêmement important de connaitre et d'adopter les processus les plus simples pour la résolution de problèmes et la prise de décision

\section{Résolution des problèmes}

La résolution des problèmes est le processus d'identification puis de mise en œuvre d'une solution à un problème. Les méthodes de résolution des problèmes s'articulent selon la démarche présentée dans le schéma 6.

Les huit étapes peuvent se résumer à quatre phases :

- $\quad$ Déterminer le problème ;

- Rechercher les causes ;

- Rechercher les solutions;

- Application et suivi.

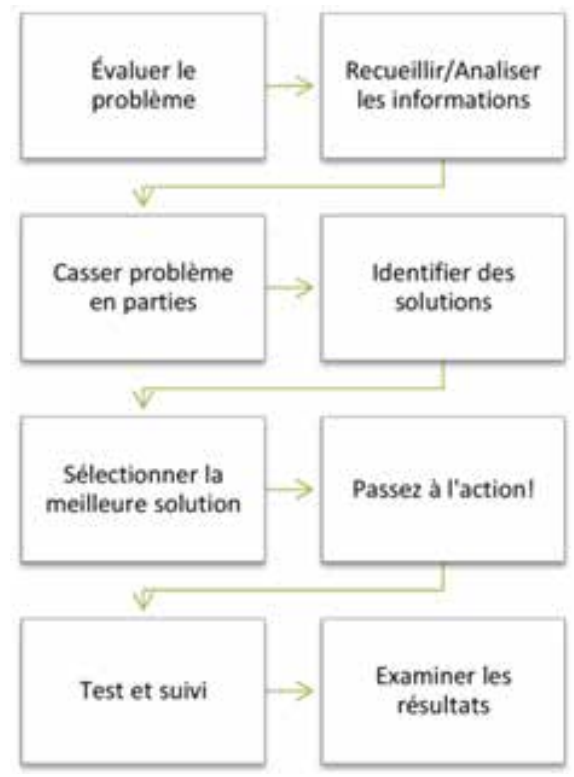

Schéma 6 - Étapes de la résolution des problèmes

\section{Prise de décision}

Une bonne prise de décision nécessite un mélange de compétences : le développement créatif et l'identification des solutions, la clarté de jugement, la fermeté de la décision et la mise en œuvre efficace. Certains techniciens ont une capacité de prise de décision plus immédiate, d'autres sont souvent en mesure de faire des évaluations

\#. Détermination des objectifs-cibles de conservation et des indicateurs

\begin{tabular}{|l|l|l|l|l|l|}
\hline $\begin{array}{l}\text { Ligne de base actuellement } \\
\text { évaluable de conservation } \\
\text { des valeurs et importance } \\
\text { de l'aire protégée à la date } \\
\text { du \#\#/\#\#/20\#\# }\end{array}$ & $\begin{array}{l}\text { Valeur de } \\
\text { référence 1 }\end{array}$ & $\begin{array}{l}\text { Valeur de } \\
\text { référence 2 }\end{array}$ & $\begin{array}{l}\text { Valeur de } \\
\text { référence 3 }\end{array}$ & $\begin{array}{l}\text { Objectif - Conditions } \\
\text { souhaitées des } \\
\text { valeurs et importance } \\
\text { de l'aire protégée à la } \\
\text { date du \#\#/\#\#/20\#\# }\end{array}$ \\
\hline 1 & & & & & \\
\hline 3 & & & & & \\
\hline
\end{tabular}



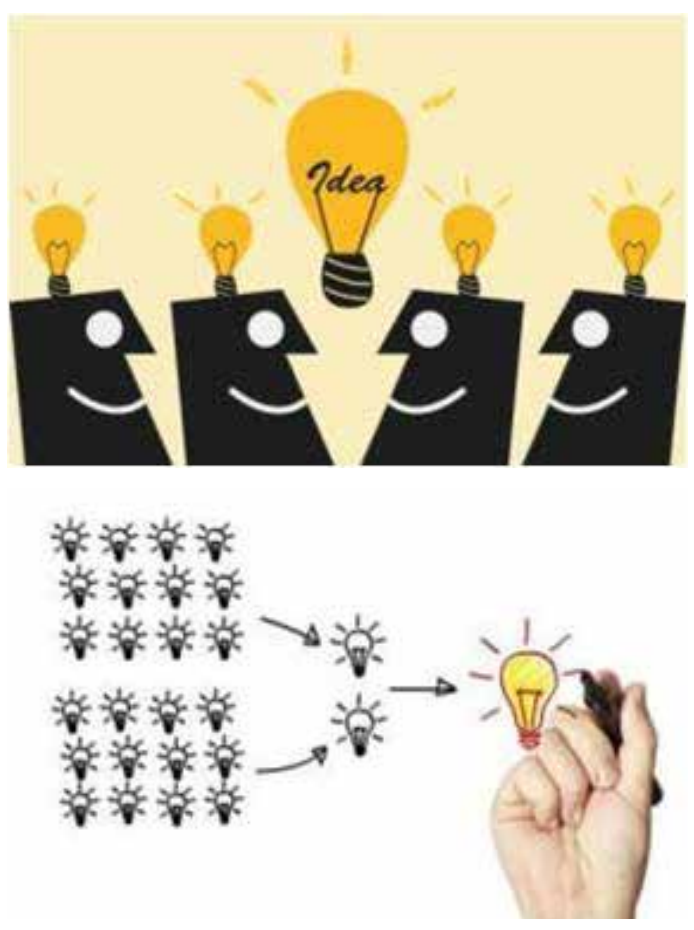

de qualité, mais doivent être plus décisifs sur les conclusions. Pour ces derniers, il existe des processus et techniques pour améliorer la prise de décision et la qualité des décisions.

Ci-dessous, les outils les plus employés pour une résolution des problèmes et une prise de décision efficace : la séance de réflexion (remue-méninges, ou brainstorming), l'arbre à problèmes, l'analyse de cause à effet, l'analyse SWOT, l'analyse PESE.

\section{La séance de réflexion (brainstorming)}

Le brainstorming est un outil de résolution de problèmes extrêmement puissant et flexible. II permet de générer un grand nombre d'idées créatives. Outre la résolution des problèmes, il peut être utilisé pour la pensée critique et la prise de décision.

\section{Arbre à problèmes}

L'arbre à problèmes permet de schématiser une situation problématique pour en analyser les causes et les conséquences. L'outil offre une image de synthèse du ou des problèmes à

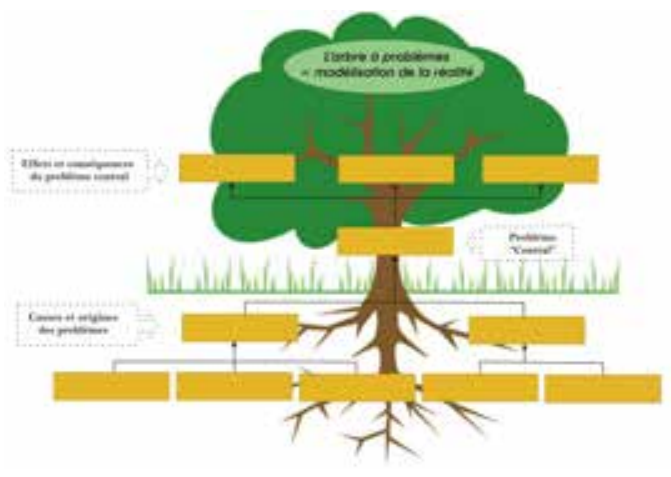

résoudre. Les causes et les conséquences sont identifiées, ce qui permet d'être lucide dans l'élaboration d'une intervention.

\section{L'analyse de cause à effet}

L'analyse de cause à effet (Root cause analysis) est une technique étape par étape, structurée, qui se focalise sur la recherche de la cause réelle des problèmes et la résolution, ou l'évitement, de ces problèmes.

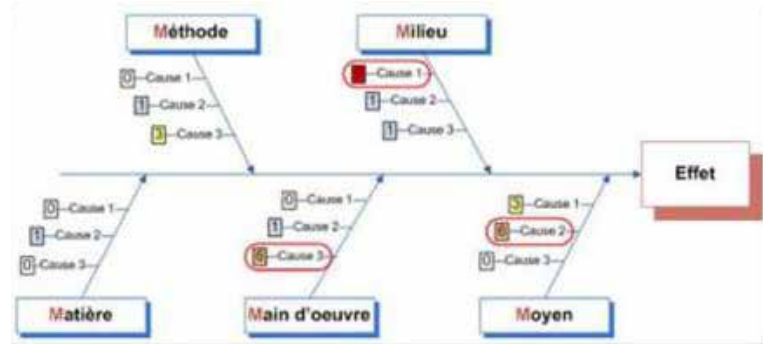

\section{L'analyse SWOT}

L'analyse SWOT ou matrice SWOT est un outil de stratégie d'intervention permettant de déterminer les options stratégiques envisageables au niveau d'un domaine d'activité. Le terme SWOT est un acronyme anglais : Strengths (forces), Weaknesses (faiblesses), Opportunities (opportunités), Threats (menaces). L'analyse SWOT permet d'évaluer les forces, les faiblesses, les opportunités et les contraintes de la gestion. Beaucoup de problèmes ont des causes profondes qui sont importantes pour leur résolution. Les symptômes sont l'indicateur d'un problème (ou de plusieurs problèmes) à résoudre.

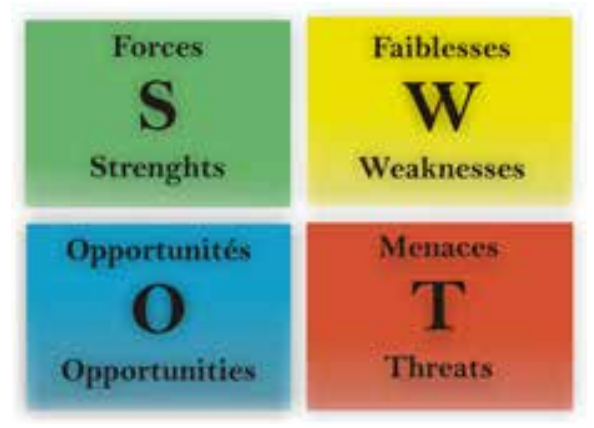

\section{L'analyse PESE (PEST)}

L'analyse PESE est l'adaptation del'analyse PEST10 (politique, économique, social, technologique) où l'analyse technologique est remplacée par l'analyse environnementale. L'analyse PESE décrit un cadre de facteurs de la gestion stratégique.

10 L'importance croissante des facteurs environnementaux ou écologiques a donné naissance à une version PEST dénommée STEER qui considère les facteurs suivants : socioculturel, technologique, économique, écologique, réglementaire. 
Elle est une partie d'une analyse stratégique et donne un aperçu des différents macro-facteurs que l'aire protégée doit prendre en considération pour ses interventions. L'analyse PESE est un outil stratégique utile pour la compréhension de la viabilité de la conservation. Comme dans un puzzle, les quatre facteurs doivent se combiner ensemble en réduisant le chevauchement d'un ou plus facteurs sur les autres.

- Les facteurs politiques comprennent des

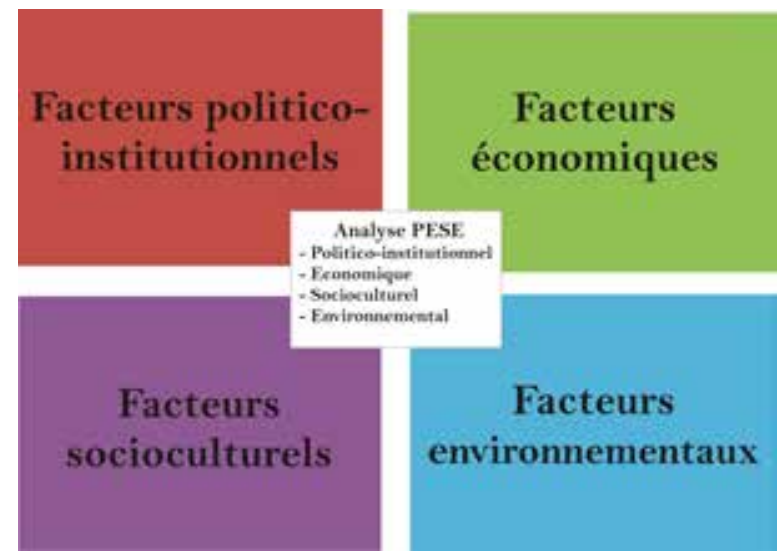

domaines tels que la politique fiscale, le droit du travail, le droit de l'environnement, les restrictions commerciales, les droits de douane, et la stabilité politique.

- Les facteurs économiques et socioéconomiques ont des répercussions majeures sur la façon dont les intervenants, dans le contexte de l'intervention de l'aire protégée, fonctionnent et prennent des décisions. Par exemple, les coûts des biens produits, l'approvisionnement, le prix des marchandises.

- Les facteurs sociaux comprennent les aspects culturels, par ex. la santé, le taux de croissance de la population, la répartition par âge, les attitudes de carrière et l'accent mis sur la sécurité.

- Les facteurs environnementaux comprennent les aspects écologiques et environnementaux pouvant notamment affecter la biodiversité et les activités dans la zone d'intervention de l'aire protégée tels que le tourisme, l'agriculture, la foresterie.

\section{Exercice 22 - Éléments prioritaires à gérer}

Cas 1 : L'analyse du contexte de l'intervention de plusieurs aires protégées a permis de définir une liste restreinte des espèces clés et des habitats sur la base desquelles établir une stratégie d'intervention. Dans le cas du PN 6, Pays 2, la base de la stratégie d'intervention était de focaliser l'action sur l'habitat - les espèces - la valorisation : 1. forêt de plaine - gorilles + chimpanzés - tourisme ; 2. savanes - cobe défassa ou waterbuck; 3. Habitats d'eau douce - crocodiles nains + hippopotames - paysages + tourisme.

\section{Questions}

1. Pourquoi doit-on définir des lignes stratégiques d'intervention ?

2. Quelles sont les démarches pour définir ces lignes stratégiques d'intervention ?

3. Le besoin de définir des lignes stratégiques en faveur du parc a été pris en compte

- en discutant avec les responsables ;

- dans l'analyse croisée entre planification et résultats-impacts ;

- dans l'analyse des menaces :

- par une autre analyse (préciser, SVP) : ..

4. Pourquoi la stratégie était-elle seulement focalisée sur trois habitats ?

Cas 2 : L'analyse du contexte de l'intervention du PN 7, Pays 4, a permis de conclure que suite au massacre des éléphants au cours de la période 2005-2010, qui a réduit la population initiale d'environ 4000 animaux à 450, la principale et seule condition de conservation souhaitée pour la sauvegarde de l'aire protégée était d'atteindre le chiffre d'environ 1000 éléphants en l'espace de 18-20 ans.

\section{Questions}

1. Pourquoi pour le PN 7 un seul objectif de gestion a-t-il été défini ?

2. Pouvons-nous considérer que le seul objectif de gestion relative à la sauvegarde de l'éléphant est suffisant pour assurer la bonne gestion du parc ?

3. L'équipe du parc devra-t-elle focaliser toutes les interventions à la faveur de la sauvegarde de l'éléphant ou bien s'agit-il d'une approche d'urgence qui doit être accompagné dans le temps par d'autres actions de gestion et gouvernance ? 


\section{FICHE 6 : Élaboration d'un indicateur}

Un élément essentiel dans la logique d'intervention « ligne de base - conditions souhaitées (de conservation) » est la définition d'un indicateur approprié. Les éléments essentiels pour l'élaboration d'un indicateur sont présentés ci-dessous.

\section{Définitions de l'indicateur}

"Un indicateur quantifie et agrège des données qui peuvent être mesurées et surveillées pour déterminer si un changement est en cours. II permet de simplifier des phénomènes en nous aidant à comprendre des réalités complexes. Les indicateurs sont sélectionnés pour fournir des informations sur le fonctionnement d'un système spécifique, dans un but spécifique (aide à la gestion, aide à la décision, aide à la communication, etc.). » http://www.dictionnaire-environnement.com.

"Les indicateurs sont essentiels à l'efficacité de tout processus de décision. Ils permettent également d'informer, de favoriser l'évaluation de l'efficacité des décisions prises en réponse à une question, et de mettre la question et les réponses en contexte. Ainsi, les indicateurs offrent une interface décisive entre la politique et les scientifiques spécialistes de la biodiversité. » Biodiversity Indicators Partnership (BIP).

\section{Étapes clés}

Les étapes pour le développement d'indicateurs couvrent trois volets :

1. Définition de l'objectif - dispositions nécessaires pour le choix et la détermination des indicateurs.

2. Processus d'élaboration - essentielle pour générer des indicateurs.

3. Déterminer les conditions pour la durabilité - mécanismes pour assurer la continuité dans l'utilisation des indicateurs.

Les étapes clés pour l'élaboration d'indicateurs de la biodiversité offrent également des avantages dans la gestion des aires protégées (AP).

\section{Dépendant d'un objectif}

Les indicateurs doivent être développés en fonction des besoins ou du but spécifique de l'utilisateur. Les indicateurs se rapportent à un objectif et sont donc « dépendants d'un objectif». La relation entre la mesure choisie comme indicateur et l'objectif de l'indicateur doit être scientifiquement/techniquement valide et facile à comprendre. Ceci est particulièrement important pour la biodiversité qui est un sujet complexe et qui est ouverte à de multiples interprétations et il est souvent difficile à traiter ou à communiquer.

L'interprétation ou la signification fournie par l'indicateur dépend de l'objet ou du sujet examiné.

\section{2. Élaboration}

\section{Question clé}

Puisque les indicateurs sont dépendants d'un objectif, leur développement ou leur sélection doit commencer par l'identification du problème à résoudre ou du support à la décision que l'indicateur doit apporter. Une question clé décrit ce que l'utilisateur ou le public souhaite comprendre à ce sujet. Quelques modèles de questions clés :

- «Quel est le statut de nos aires protégées ?

- "Quels sont les bénéfices fournis par l'AP aux communautés locales ?»

- «Quelles sont les priorités de gestion pour nos aires protégées ? »

\section{Modèle conceptuel}

Pour aider à déterminer et expliquer la relation entre un indicateur et son objectif, il est très utile d'élaborer un modèle conceptuel du sujet d'intervention et de la problématique à résoudre. Le point de départ dans la production d'un modèle conceptuel est la question clé formulée pour vérifier la correspondance entre les indicateurs et les objectifs de gestion identifiés. Un modèle conceptuel est globalement un diagramme ou un schéma qui représente les principaux sujets de préoccupation et la façon dont ceux-ci sont liés 
les uns aux autres. Le diagramme ou schéma permet de clarifier le sujet traité pour tous les intéressés et facilite la communication sur les interventions et les indicateurs adoptés. II aide à évaluer : i) la pertinence des indicateurs potentiels et leur capacité à répondre à la ou aux question(s) clé ; et ii) la validité scientifique/ technique, compte tenu de la façon dont le/les indicateur(s) représente(nt) les sujets d'intervention et les changements suite aux interventions de conservation. Un bref texte d'accompagnement peut compléter et donner plus d'explications au schéma du modèle conceptuel.

Un schéma conceptuel peut être confondu avec les cadres analytiques et de présentation comme celui de Pression-État-Réponse (PER). La différence entre eux est essentiellement une question d'échelle, car il y a un certain chevauchement dans leur utilisation. Les cadres analytiques tels que le cadre PER sont un guide très large pour aider à organiser les questions clés et l'analyse d'un vaste sujet comme l'état de l'environnement. Un diagramme de modèle conceptuel des indicateurs est une représentation plus détaillée des questions spécifiques résultant du traitement d'une question clé. Une question clé très générale peut être étudiée d'abord avec un modèle conceptuel général de la question, qui permet d'offrir un aperçu, puis à l'aide de modèles plus détaillés des questions plus spécifiques.

\section{Narratif}

Un aspect important à retenir lors de l'élaboration ou de la sélection des indicateurs, est de considérer son élaboration en termes d'une " histoire " ou d'un " texte narratif " que vous voulez transmettre à l'utilisateur. Cette étape du processus d'élaboration doit permettre de vérifier si l'indicateur répond à la question clé, de fournir des éléments explicatifs, de communiquer l'évolution du phénomène à observer et d'entamer les interventions pour atteindre un objectif.

\section{Composition}

Une fois que toutes les indications conceptuelles sont disponibles, on doit passer à la composition des différents éléments qui constituent un indicateur et qui assurent son utilisation. La fiche annexe page 128 permet de synthétiser et d'afficher le résultat final de l'élaboration de l'indicateur. Les éléments à fournir sont précisés ci-dessous, et les éléments complémentaires sont en italique :

- Nom de l'indicateur ;

- Agence de gestion ;

- Question(s) clé auquel l'indicateur permet de répondre ;

- Modèle conceptuel ;

- Objectif ;

- Effets/impacts attendus ;

- Résultats - Produits ;

- Activités ;

- Méthodologie et méthode de calcul ;

- Description des données sources ;

- Unités dans lesquelles elle est exprimée ;

- Utilisateurs de l'indicateur ;

- Échelle de l'utilisation appropriée ;

- Limites à l'utilité et à la précision ;

- Mise à jour de l'indicateur ;

- Ligne de base ;

- Conditions souhaitées ;

- Valeurs de référence indicatives ;

- Visualisations les plus efficaces de présentation ;

- Potentiel pour l'agrégation ;

- Signification de tendances à la hausse ou à la baisse ;

- Raisons possibles de tendances à la hausse ou à la baisse ;

- Indicateurs étroitement liés ;

- Informations complémentaires et commentaires. 


\section{Informations}

II y a presque toujours des données pertinentes disponibles pour commencer à produire des indicateurs de biodiversité. II est indispensable de connaitre les données utilisées - leur fiabilité, leurs limites, la source, etc. Les mêmes données peuvent être utilisées dans un indicateur à des fins multiples ou pour plusieurs indicateurs.

\section{Durabilité}

Les mécanismes pour assurer la durabilité dans l'utilisation des indicateurs sont multiples et ne dépendent pas exclusivement des ressources financières.

Un indicateur efficace doit être :

- Scientifiquement fondé :

A. puisqu'il y a une connexion acceptée entre l'indicateur et son objectif, avec un consensus que tout changement de l'indicateur entraînera un changement dans la valeur de l'objectif ;

B. les données utilisées sont fiables et vérifiables.

- Produit au fil du temps, selon les données disponibles ;

- Représentatif des changements de l'objectif ;

- Facilement compréhensible :

A. conceptuellement : de la façon dont la mesure se rapporte à l'objectif ;

B. dans sa présentation;

C. pour l'interprétation des données.

- Appropriés aux besoins de l'utilisateur ;

- Utilisé pour mesurer les progrès, fonctionner en alerte rapide en cas de problème, comprendre un problème, élaborer des rapports, accroître la conscientisation, etc.

\section{Typologie}

Du point de vue des cibles à atteindre, les indicateurs possibles à utiliser dans les interventions de conservation des aires protégées peuvent être classifiés en quatre typologies :

1. Indicateurs de gestion (permettent de suivre l'évolution des orientations de gestion fournies par l'objectif général ou les objectifs stratégiques) ;

2. Indicateurs de conservation (mesurent l'évolution de la réalisation des effets/impacts définis au niveau stratégique et liés à l'orientation de la gestion) ;

3. Indicateurs de résultats - produits (mesurent quantitativement et qualitativement la nature et le niveau du résultat à atteindre) ;

4. Indicateurs d'activités ou des biens et services produits (mesure du volume de travail s'exprimant en unités).

Dans le cas de la gestion des aires protégées, les indicateurs relatifs aux objectifs et aux effets/ impacts sont les plus utilisés.

Du point de vue de leur affichage, la plupart des indicateurs de la biodiversité peuvent être classés en deux types fondamentaux :

- Indicateurs spatiaux basés sur des cartes ou des représentations spatiales ;

- Indicateurs basés sur des indices ou des graphiques.

Les indicateurs cartographiques séduisent souvent les utilisateurs. Cependant, puisque les élaborations SIG sont relativement nouvelles et chères, les jeux de données cartographiques ne sont pas toujours disponibles en séries chronologiques. Néanmoins, les cartes peuvent être utiles comme références permettant de mesurer les changements. 


\section{Conclusion}

Les intervenants dans la conservation sont diversifiés et dispersés dans une grande variété de secteurs. Cependant, ils ont un problème commun : les interventions manquent souvent d'objectifs clairement définis et de mécanismes pour mesurer les progrès, de sorte que la définition des besoins du monde de la conservation n'est pas toujours simple. Dans ce cas, les indicateurs peuvent aider à une meilleure définition des objectifs et des stratégies de conservation et ainsi sensibiliser et faciliter la compréhension des décideurs pour l'obtention d'appuis futurs. Les indicateurs sont un aspect fondamental des interventions de conservation, et leur définition requiert une analyse détaillée.

L'accès à des indicateurs clairs et facilement compréhensibles à tous a fait ses preuves, et s'avère être la solution la plus efficace pour communiquer sur un sujet difficile comme la conservation de la biodiversité auprès du grand public.
II est important de préciser qu'un indicateur ne pourra jamais représenter tout ce que vous voulez observer, puisque l'indicateur montre souvent une situation plus complexe.

Les indicateurs font partie d'un processus et doivent conduire à des décisions éclairées. Ils ne sont donc pas des fins en soi.

\section{Sources :}

Guidance for National Biodiversity Indicator Development and Use - www.bipnational.net et intégration par le projet Biopama)

Partenariat relatif aux indicateurs de la biodiversité. (2011) Guide de développement et d'utilisation des indicateurs nationaux de la biodiversité. Centre mondial de suivi de la conservation du PNUE, Cambridge, R-U. 40pp

Schéma synthétique du formulaire BIOPAMA, élément d'analyse de l'évaluation de l'efficacité de la gestion des aires protégées

Les indicateurs de gestion et de conservation doivent normalement se référer à la partie relative au contexte de la gestion.

\begin{tabular}{|c|c|c|c|}
\hline \multirow{2}{*}{$\begin{array}{l}\text { CONTEXTE DE LA } \\
\text { GESTION }\end{array}$} & \multicolumn{3}{|c|}{ PART ACTIVE } \\
\hline & \multicolumn{2}{|c|}{$\begin{array}{c}\text { ÉLÉMENTS SPÉCIFIQUES DU } \\
\text { PROCESSUS }\end{array}$} & \\
\hline $\begin{array}{l}\text { - Gouvernance } \\
\text { - Contrainte ou soutien par } \\
\text { l'environnement politique et } \\
\text { civil externe }\end{array}$ & $\begin{array}{l}\text { - Relations avec les parties } \\
\text { prenantes }\end{array}$ & $\begin{array}{l}\text { - Suivi/ } \\
\text { recherche }\end{array}$ & \multirow{7}{*}{$\begin{array}{l}\text { - PLANIFICATION } \\
\text { - INTRANTS } \\
\text { - PROCESSUS } \\
\text { (Systèmes et processus } \\
\text { de gestion interne) }\end{array}$} \\
\hline \multicolumn{3}{|l|}{ - Classifications } & \\
\hline - Espèces clés & - Protection et gestion & \multirow{5}{*}{$\begin{array}{l}\text { - Suivi/ } \\
\text { recherche }\end{array}$} & \\
\hline - Habitats & - Protection et gestion & & \\
\hline - Changement climatique & $\begin{array}{l}\text { - Gestion des effets du } \\
\text { changement climatique } \\
\text { et des services } \\
\text { écosystémiques } \\
\end{array}$ & & \\
\hline - Services écosystémiques & $\begin{array}{l}\text { - Gestion des effets du } \\
\text { changement climatique } \\
\text { et des services } \\
\text { écosystémiques } \\
\text { - Gestion du tourisme } \\
\end{array}$ & & \\
\hline - Menaces & $\begin{array}{l}\text { - Relations avec les parties } \\
\text { prenantes } \\
\text { - Protection et gestion }\end{array}$ & & \\
\hline
\end{tabular}




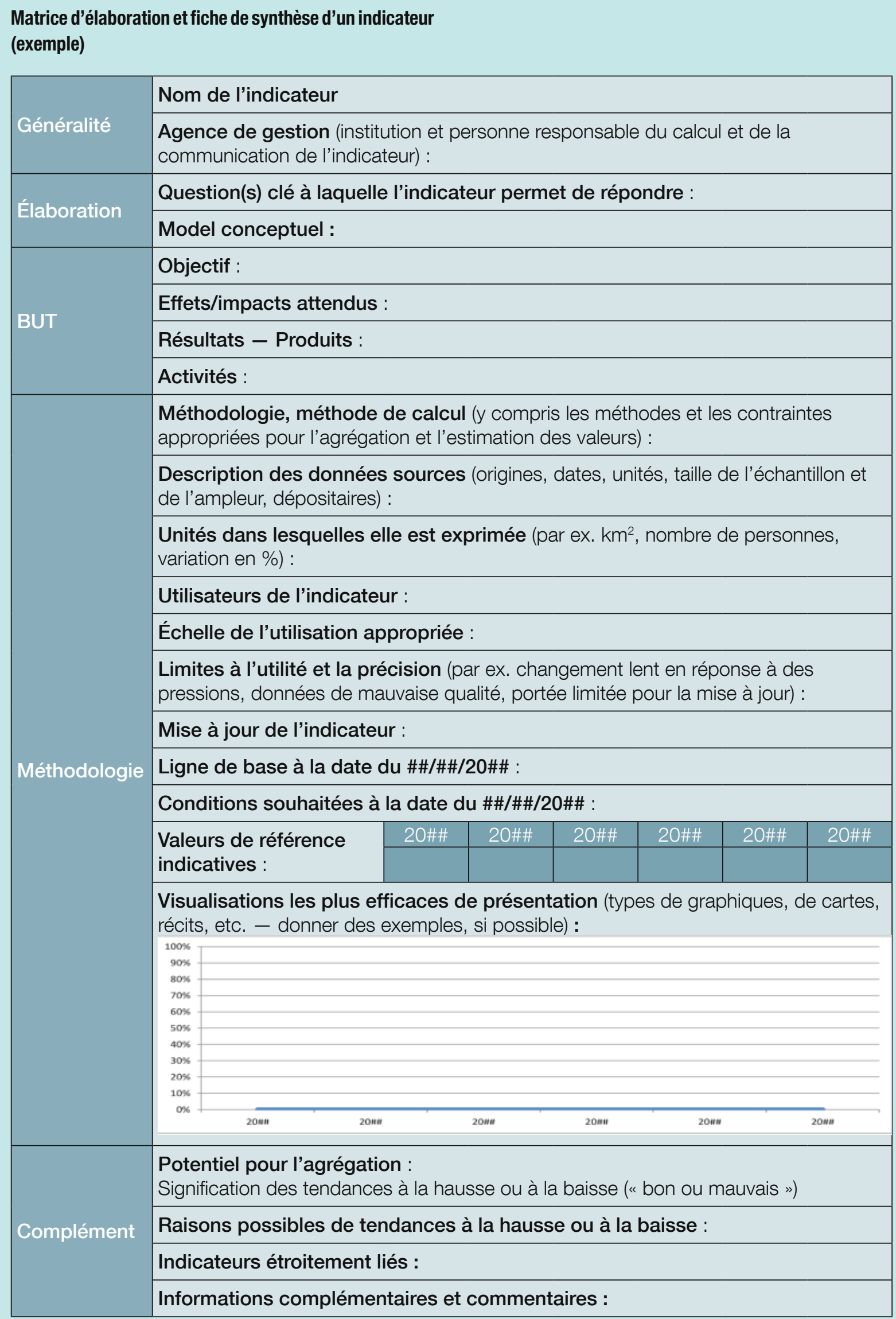




\subsubsection{Des propositions : où, pour qui, comment?}

Les recommandations sont destinées en premier lieu à l'équipe de gestion elle-même afin de mettre à jour le plan de gestion et/ou le plan de travail annuel de l'aire protégée. II est cependant important d'estimer dans quelle mesure certaines de ces recommandations sont urgentes, ou nécessitent des discussions avec des échelons supérieurs ou des partenaires externes avant d'être décidées.

II existe différentes méthodes pour communiquer les résultats et les propositions. Le coach doit discuter étroitement avec le Conservateur, les responsables de la planification et du suiviévaluation de l'aire protégée (au niveau du site et au niveau central) pour déterminer comment présenter les propositions. Les options, souvent compatibles, sont :

- Un rapport verbal ;

- Un document Powerpoint, surtout si le public est nombreux (plus de 20 personnes) ;

- Les rapports écrits : très importants pour la mémoire de l'organisation et pour servir de futures références.

\subsubsection{Organiser la restitution}

La restitution " officielle " peut constituer un sérieux défi pour de nombreux gestionnaires d'aire protégée. En effet, elle peut être vue comme la proclamation des résultats devant un groupe. Le coach aura pour mission de faciliter cette étape, notamment en décontractant l'atmosphère pour qu'elle soit propice aux discussions, aux réflexions et à la collaboration.

La restitution, qu'elle soit au niveau du site ou au niveau central, doit comprendre un certain nombre de composantes standards :

- Un bref rappel du contexte de l'évaluation, pourquoi celle-ci a été réalisée, la méthodologie utilisée et les personnes consultées ;

- Un résumé des données et de l'analyse, notamment la visualisation par le radar ;

- Une analyse claire qui décrit comment l'évaluation a été faite ;

- Des recommandations détaillées.

La restitution doit ensuite permettre au public de poser des questions et d'émettre des remarques ou des recommandations. Le coach jouera le rôle de facilitateur afin de faire entendre tous les points de vue.

\section{Encadré 14 - Des recommandations constructives}

Les recommandations doivent:

- S'assurer que tous les conseils sont suffisamment clairs et spécifiques pour améliorer les pratiques de la conservation, et assez réalistes pour garantir que l'on trouvera des solutions faisables pour les problèmes prioritaires.

- Comprendre les priorités à court et à long terme, un calendrier et un budget (avec les besoins de fonds supplémentaires, si nécessaire). Les activités pour le court terme doivent être claires, concrètes, réalisables dans les délais impartis et avec les ressources disponibles, et classées par ordre de priorité. Les recommandations à long terme doivent identifier les changements de ressources et de politiques nécessaires à leur mise en œuvre.

- Avoir un effet retour sur les systèmes de gestion pour influencer les planifications futures, l'attribution des ressources et les activités.

- Se focaliser d'abord sur les activités des gestionnaires et des gardes mais, si nécessaire, identifier aussi les réponses requises au-delà des limites du parc.

- Être contrôlées par les plans de travail annuels et par les évaluations suivantes, pour vérifier si les activités identifiées ont été réalisées et aussi (ce n'est pas la même chose) si elles ont relevé les défis correspondants.

Hockings, M., Stolton, S., Leverington, F., Dudley, N. et Courrau, J. (2008). Évaluation de l'efficacité : Un cadre pour l'évaluation de l'efficacité de la gestion des aires protégées, 2ème édition. Gland, Suisse : UICN. p.40 


\section{Liens web et bibliographie}

Classification UICN : aires protégées

Les différents statuts des AP : Zones importantes pour les oiseaux, Zones importantes pour la biodiversité.

Liste rouge de I'UICN

http://www.iucnredlist.org/

Liste CITES des espèces protégées

http://www.cites.org/

Évaluation de l'efficacité de la gestion des aires protégées

www.cbd.int/protected-old/PAME.shtml

\section{Fichiers électroniques de référence}

1-1Catégories UICN de gestion des AP

1-4No.10 Lignes directrices pour la planification de la gestion des AP

1-9Triplet (2009) Manuel de gestion des AP francophones

2-3 Hockings et al (2008) Évaluation de l'efficacité Un cadre pour l'évaluation de l'efficacité de la gestion des aires protégées

2-4 Leverington et al (2008) Management

effectiveness evaluation in protected areas - a global study

2-5 Leverington et al (2008) Management effectiveness evaluation in protected areas - a global study Overview of approaches and methodologies 2-6 Leverington et al (2010) Management effectiveness evaluation in protected areas - a global study. $2^{\text {nd }}$ Edition.

\section{En cas de difficulté}

Le binôme du coach peut avoir recours aux points focaux

\section{Afrique Centrale}

Jean Nestor Bouengue (Gabon)

nestorboue@yahoo.fr

Radar Nishuli Birhashirwa (RDC)

radarnishu@yahoo.fr

\section{Afrique de l'Ouest}

Comlan Aristide Tehou (Bénin)

tehouaristidecomlan@yahoo.fr tehouaristide@gmail. com tehouaristide@hotmail.com

Pour les questions relatives au remplissage du Formulaire :

Carlo Paolini

carlopaolini@yahoo.it 


\section{PARTIE 4}

\section{Encodage des données dans le formulaire biopama}

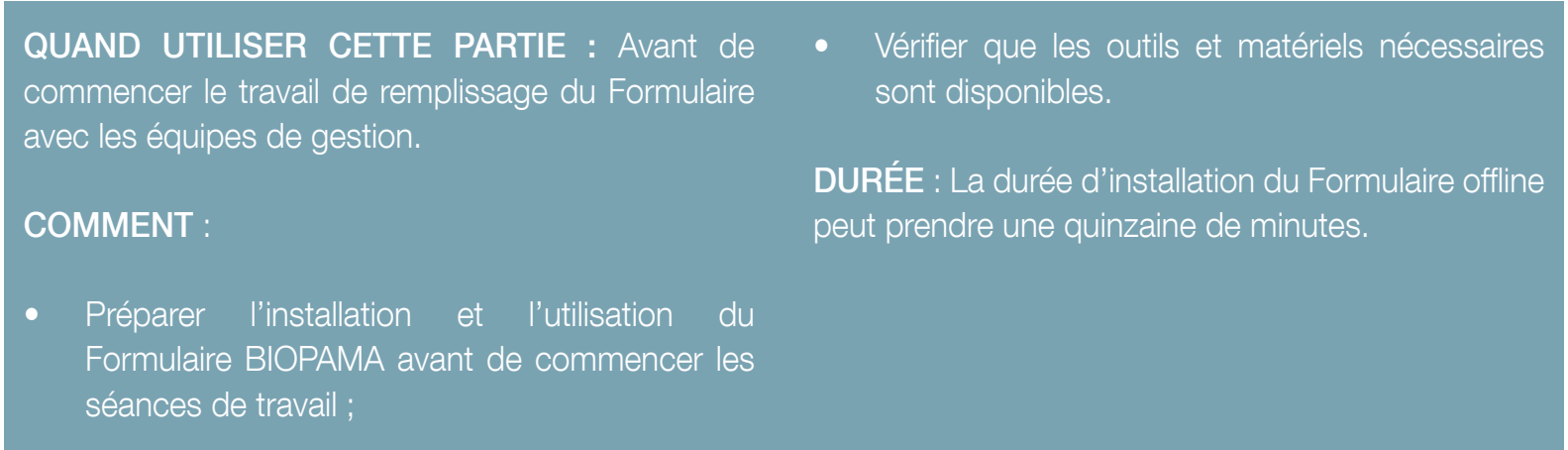

\subsection{Introduction}

Cette partie se focalise sur les aspects liés à la manipulation informatique du Formulaire. Les questions de compréhension des indicateurs à renseigner sont détaillées dans la partie 3.

II est fortement recommandé de transcrire les données sur l'aire protégée à travers le Formulaire BIOPAMA disponible en ligne, afin d'éviter le double travail de ré-encodage en ligne avec les données collectées à partir de la version hors-ligne (offline) ainsi que d'éventuelles pertes de données dans les ordinateurs privés des gestionnaires ou des coaches.

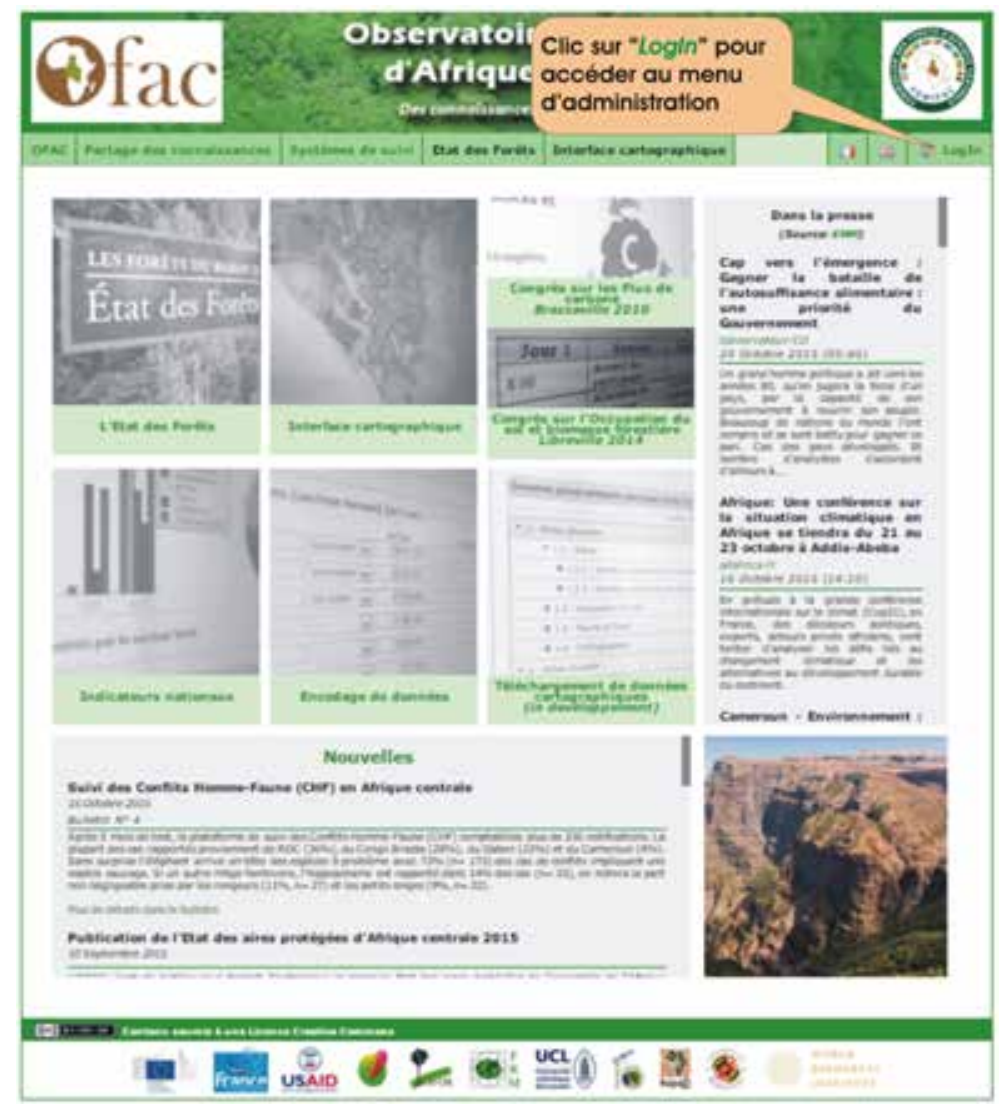

Schéma 7 - Page d'accueil du site de l'OFAC 


\subsection{Accès au formulaire}

Les coaches disposent de deux méthodes pour remplir le Formulaire BIOPAMA.

- À travers le Formulaire en ligne (online) ;

- À travers le Formulaire hors ligne (offline), qui requiert l'installation d'une application qui aura été préalablement remise aux coaches.

\subsubsection{Formulaire en ligne (online)}

Cette section présente les étapes d'utilisation du Formulaire en ligne.

\section{Étape 1: Accès au site de l'OFAC}

Allez à la page d'accueil du site de l'Observatoire, à l'adresse : http://www.observatoire-comifac.net. La page suivante s'affiche :

\section{Étape 2 : Connexion à la base de données}

\section{(7) observafoire des Forèts d'Afrique centrale Acministration Area}

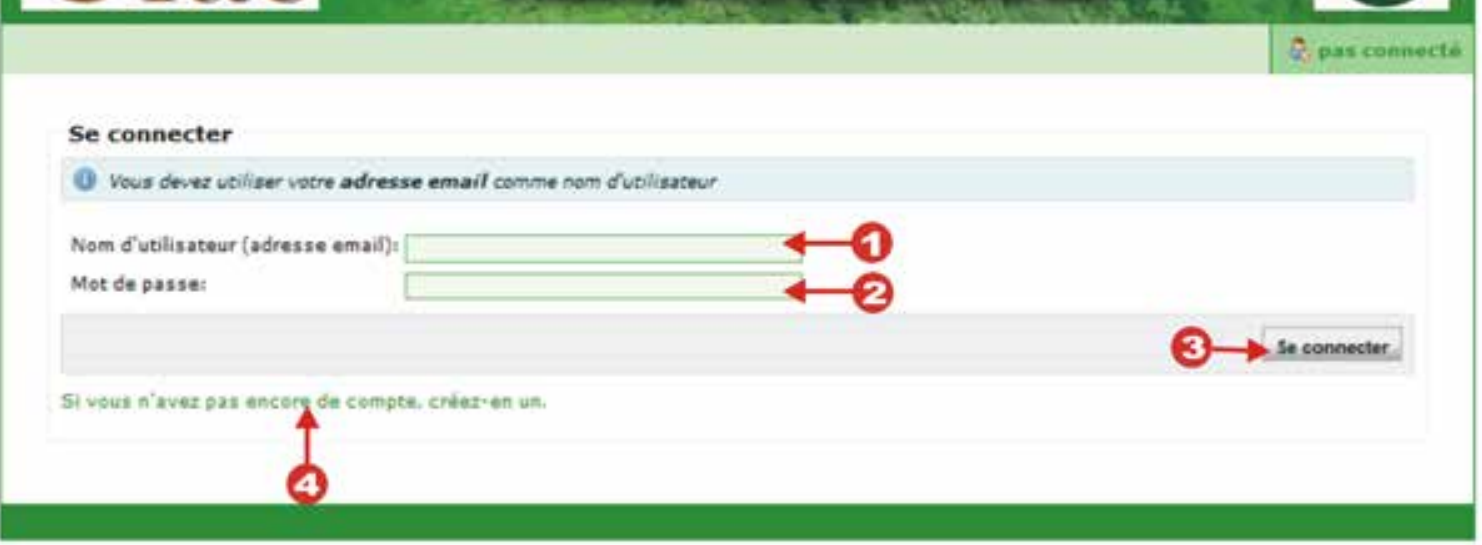

Schéma 8 - Page de connexion en ligne

- Cliquez sur le lien « Logln» dans la partie supérieure droite de la page d’accueil (cf. Schéma 7). La page de connexion permet d'une part l'accès au menu d'administration, et d'autre part d'initier la création d'un compte OFAC.

- Saisissez votre Nom d'utilisateur (cf. Schéma 8[1]), votre Mot de passe (cf. Schéma 8[2]) et cliquez sur le bouton Se connecter (cf. Schéma 8[3]).

- Si la connexion est réussie, le menu principal d'administration s'affiche (cf. Schéma 9).

- Dans le menu principal d'administration, les liens d'accès aux différentes catégories de formulaires sont visibles en fonction des droits d'accès dont dispose l'utilisateur. Les liens «Projets ", "Experts " et "Formations » sont visibles par défaut à tout utilisateur sans avoir besoin de droits d'accès.

- Le lien «Les aires protégées » qui concerne ce manuel est un lien sécurisé qui demande à l'utilisateur d'avoir un droit d'accès pour y accéder. Ainsi, si l'utilisateur ne trouve pas le lien «Les aires protégées » dans le menu principal d'administration, il devra contacter l'administrateur de la base de données afin que les droits lui soient accordés (voir les coordonnées à la fin du présent manuel).

Note : Si l'utilisateur n'a pas encore un compte dans la base de données de l'OFAC, il peut en créer un en cliquant sur le lien «Si vous n'avez pas encore de compte, créez-en un " (Voir 4.4 : Comment créer et gérer son compte?). 


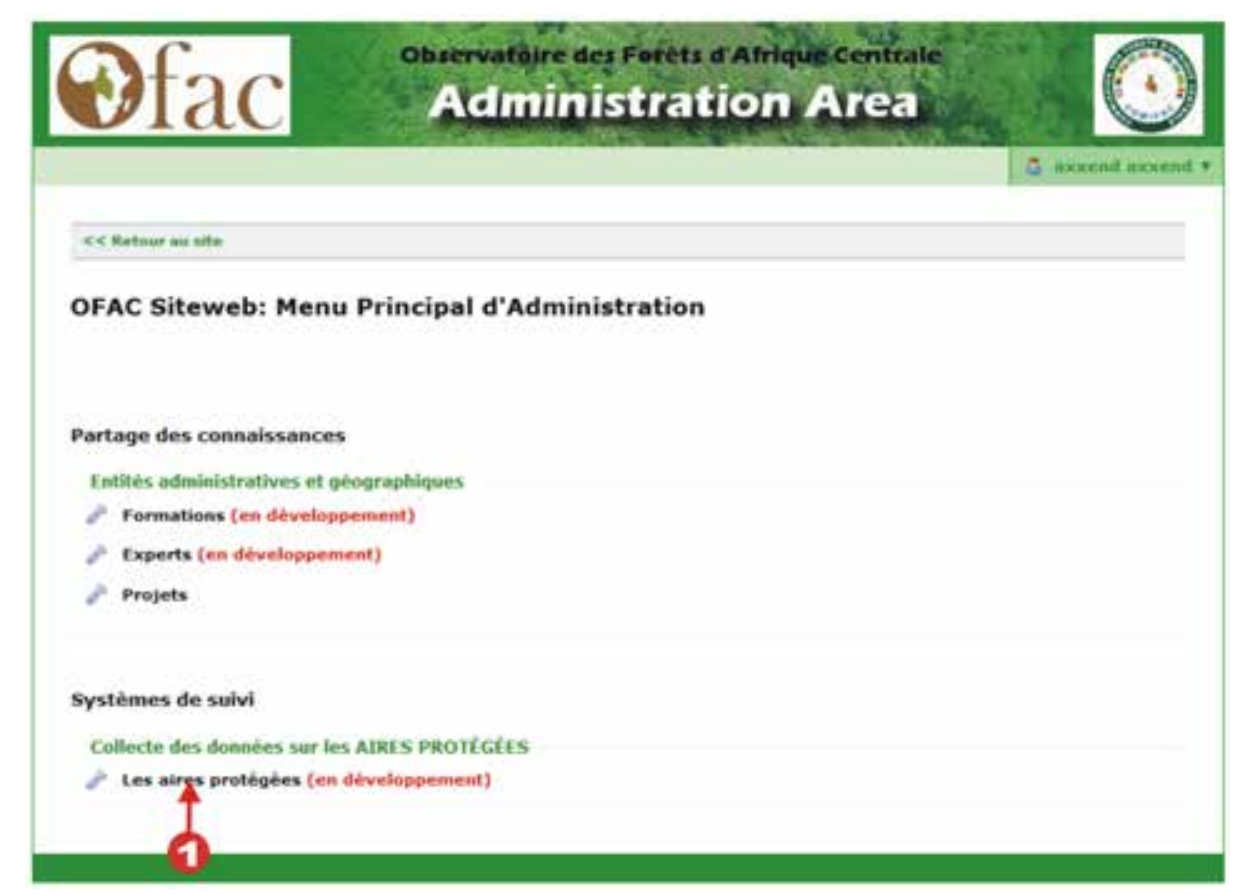

Schéma 9 - Menu principal d'administration

\subsubsection{Formulaire hors ligne (offline)}

La version hors ligne a été développée afin de pallier au problème de connexion Internet dans certaines aires protégées. Ainsi, si l'aire protégée dispose d'une connexion Internet fiable, cette application ne lui sera pas utile.

Cette section donne la démarche à suivre pour l'installation et l'utilisation de l'application.

\section{Étape 1 : Installation de l'application}

L'utilisateur recevral'application sous la forme d'un fichier ZIP. Ainsi pour pouvoir exploiter l'ensemble des éléments contenus dans le fichier ZIP, l'utilisateur doit décompresser l'archive. Windows XP, Vista, 7 et 8 contiennent un assistant de compression/décompression de fichiers ZIP qui peut simplifier la tâche.

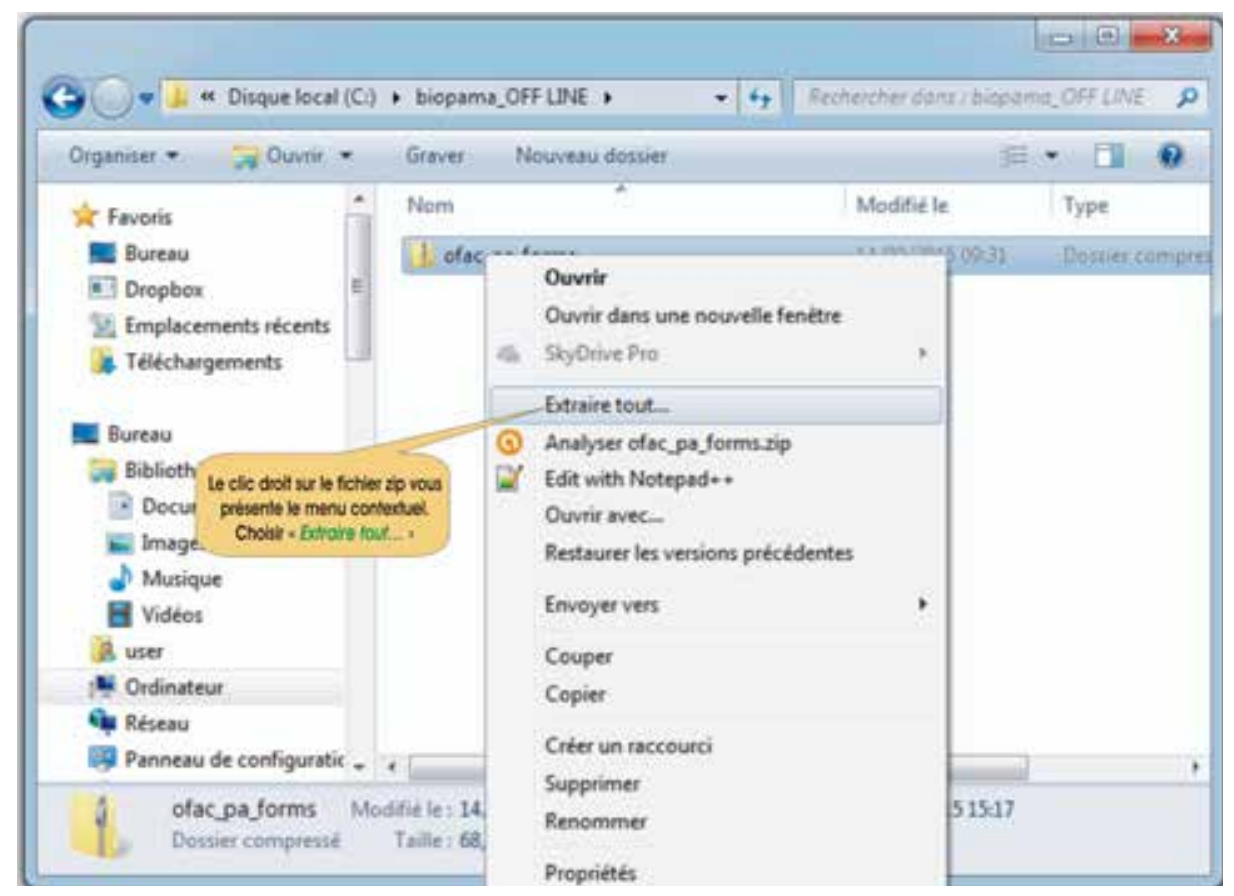

Schéma 10 - Extraction du fichier ZIP 
- Faites un clic droit sur le fichier ZIP, choisissez «Extraire tout ... » et suivez les instructions.

- Au cours du processus d'extraction, l'utilisateur doit choisir un endroit approprié sur son ordinateur où les fichiers seront extraits. II est important de noter que chacun des fichiers est indispensable pour le bon fonctionnement de l'application. Ainsi, il est conseillé d'extraire l'ensemble de ces fichiers dans un endroit sécurisé où les manipulations sont réduites (cf. Schéma 11).

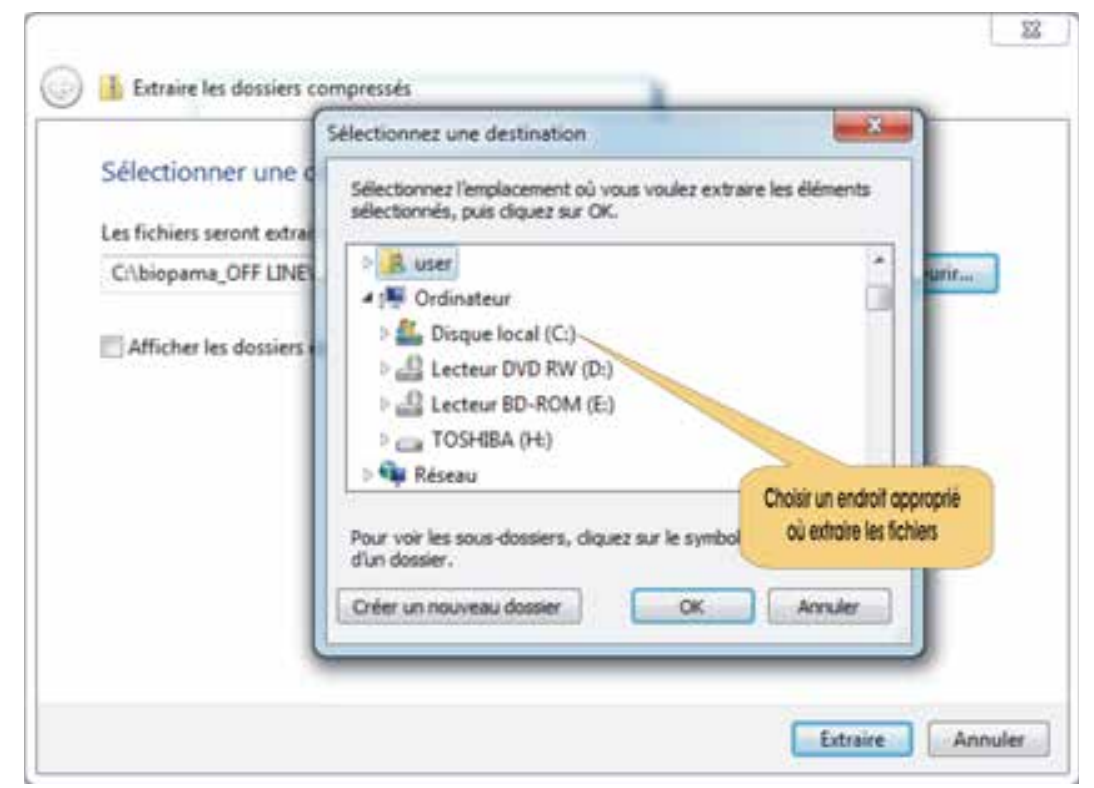

Schéma 11 - Choix de l'emplacement du dossier

Une fois l'extraction terminée, l'application est prête à être utilisée. Mais, afin d'éviter d'éventuelles suppressions de fichiers dans le répertoire qui rassemble l'ensemble des fichiers de l'application, nous vous conseillons de créer un raccourci à partir du menu Démarrer sur votre bureau. Dès lors, l'utilisateur pourra lancer l'application directement à partir du raccourci créé sur son bureau, sans rentrer dans le dossier contenant les fichiers qui constituent l'application. Pour créer un raccourci bureau, suivre les étapes du schéma 9.

: Dans le cadre de l'utilisation du Formulaire hors ligne, il peut arriver que l'utilisateur ne se sente pas à l'aise avec l'espace de travail proposé par Desktop chrome. Il peut ainsi se rendre dans son navigateur par défaut en faisant un clic droit dans un espace vide et en choisissant "Open page in external browser » tout en laissant le premier espace de travail et la console tourner.

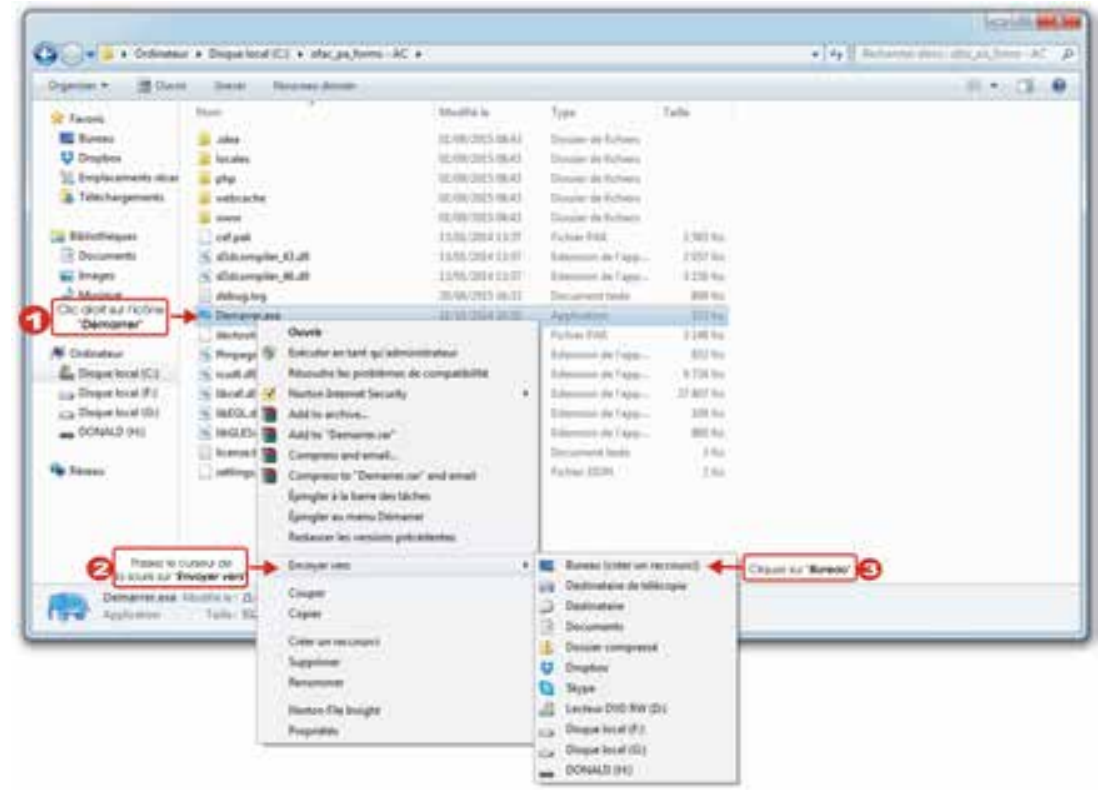

Schéma 12 - Création du raccourci sur le bureau 
II convient de noter que, pour les utilisateurs ayant installé sur leur ordinateur un logiciel de compression/ décompression de fichier tel que WinZip, WinRar, 7-Zip, le fichier compressé apparaitra avec l'icône de l'utilitaire en question. Ces utilisateurs doivent utiliser ce logiciel en suivant les étapes de décompression susmentionnées pour extraire l'application.

\section{Étape 2 : Utilisation de l'application}

- Cliquez sur l'icône du menu Démarrer.

- Deux fenêtres s'ouvrent : un serveur console qui tournera en arrière-plan et l'application elle-même. Les deux doivent toujours fonctionner simultanément pendant l'utilisation de l'application.

- L'application hors ligne s'ouvre directement sur la page de connexion (cf. Schéma 10). Tout utilisateur a besoin d'un compte (profil d'utilisateur) pour pouvoir avoir accès aux formulaires.

- L'application vous sera remise avec un profil d'utilisateur par défaut déjà créé qui vous permettra d'accéder pour la première fois au menu d'administration. Ce profil devra être modifié en utilisant vos propres informations (Voir 4.4 : Comment créer et gérer son compte ?)

Vous serez orienté vers la page de connexion (cf. Schéma 10), qui est identique à la page de connexion en ligne (cf. Schéma 5)

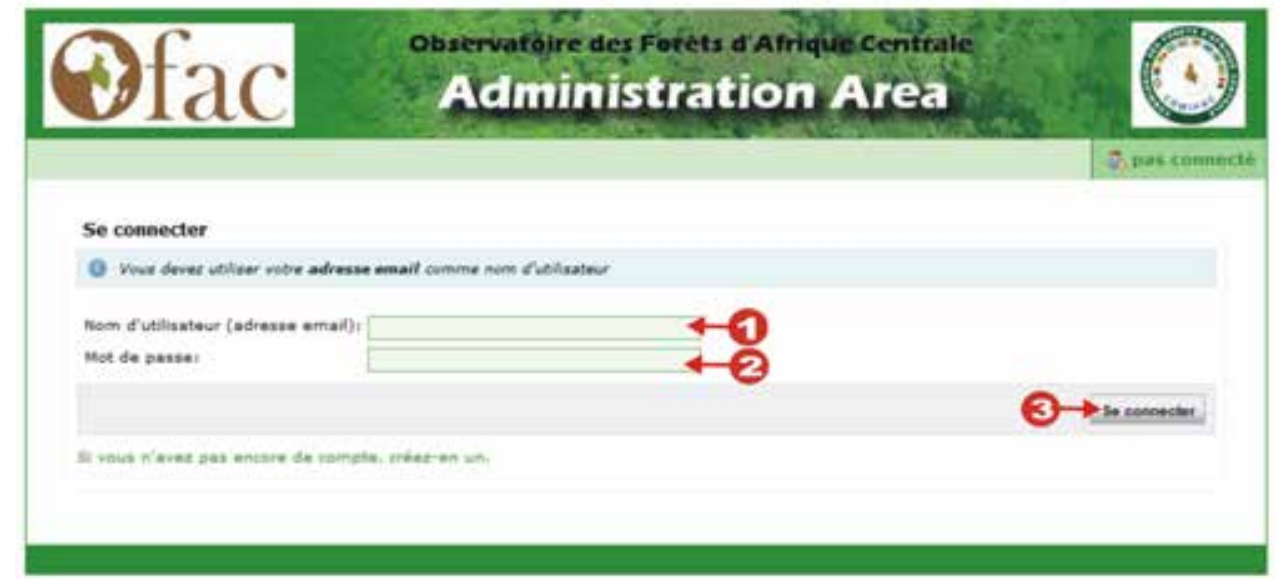

Schéma 13 - Page de connexion hors ligne

- Saisissez votre Nom d'utilisateur (cf. Schéma 13, [1]), votre Mot de passe (cf. Schéma 13, [2]) et cliquez sur le bouton Se connecter (cf. Schéma 13, [3]).

- Si la connexion est réussie, le menu principal d'administration s'affiche (cf. Schéma 9)

\subsection{Encodage des données}

Qu'il utilise le Formulaire en ligne ou hors ligne, l'utilisateur doit partir du menu principal d'administration (Schéma 14) pour commencer à encoder les données.

IMPORTANT : Le processus est le même qu'il s'agisse du Formulaire en ligne ou hors ligne.

\subsubsection{Gestion du Formulaire}

Une fois le pays sélectionné, la liste des formulaires d'aires protégées déjà créés pour ce pays se déploie (cf. Schéma 5)

- Cliquez sur « Les aires protégées » (cf. Schéma 14 [1]) pour se rendre dans l'espace réservé aux formulaires Aires Protégées.

- Dans cet espace, les formulaires sont regroupés par pays. L'utilisateur doit sélectionner un pays pour afficher la liste des formulaires d'aires protégées préalablement encodés pour ce pays (cf. Schéma 15) 


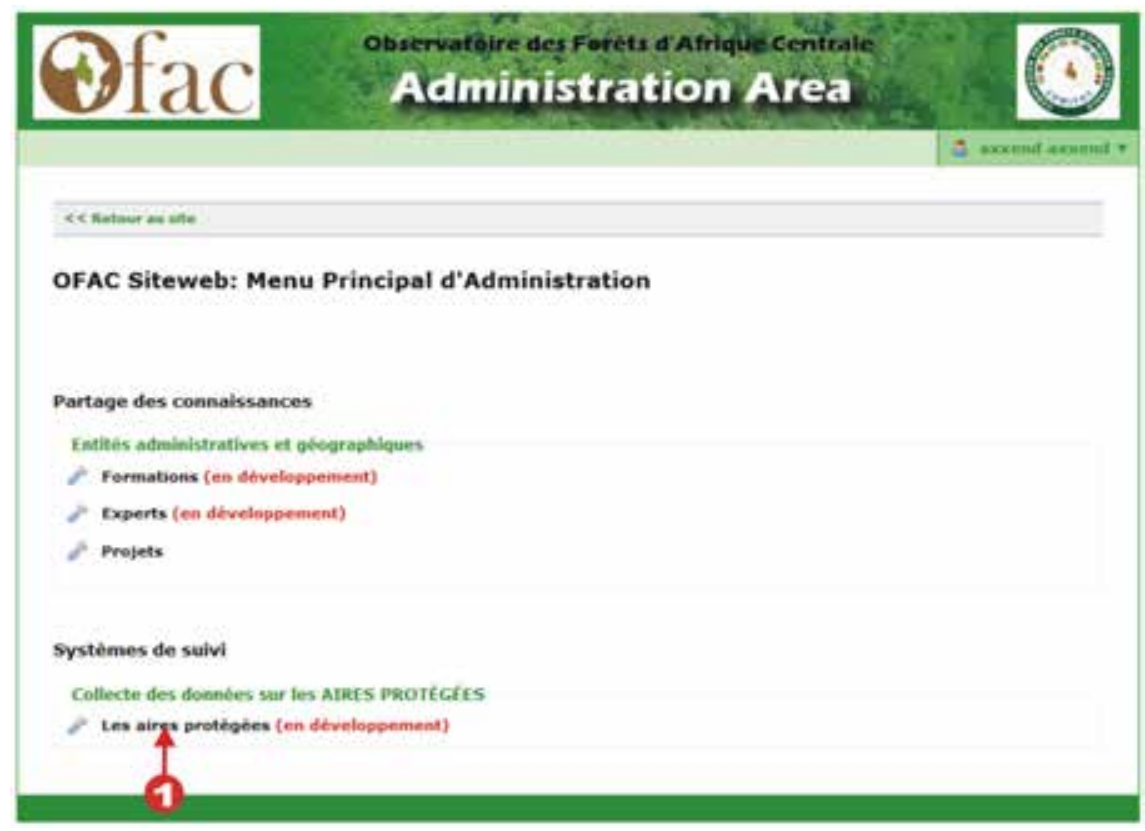

Schéma 14 - Menu principal d'administration

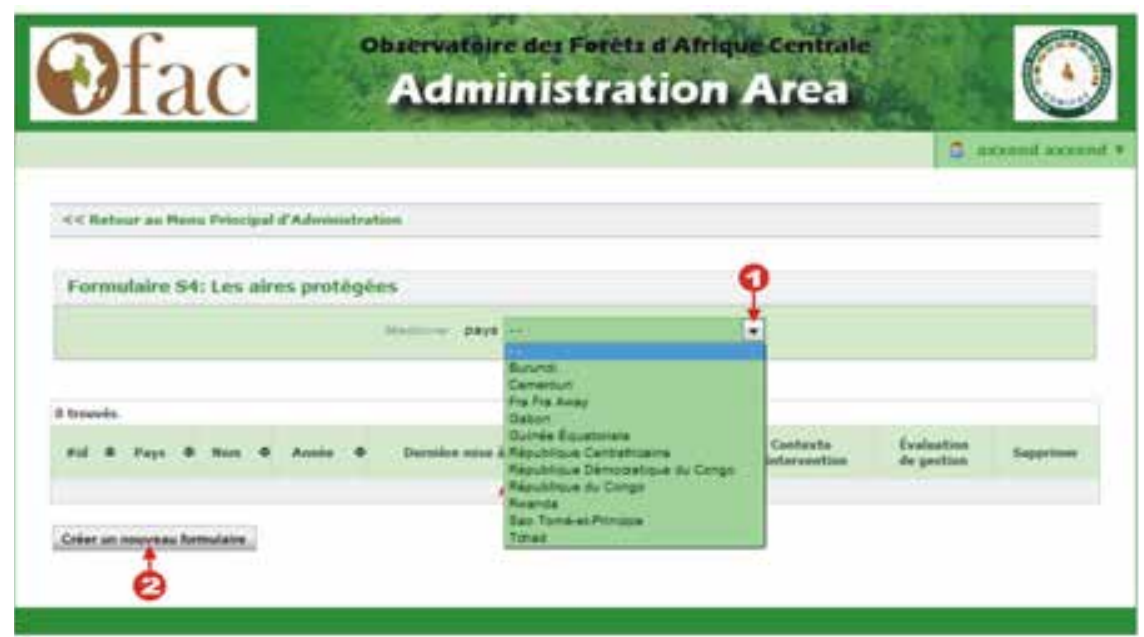

Schéma 15 - Sélection de pays
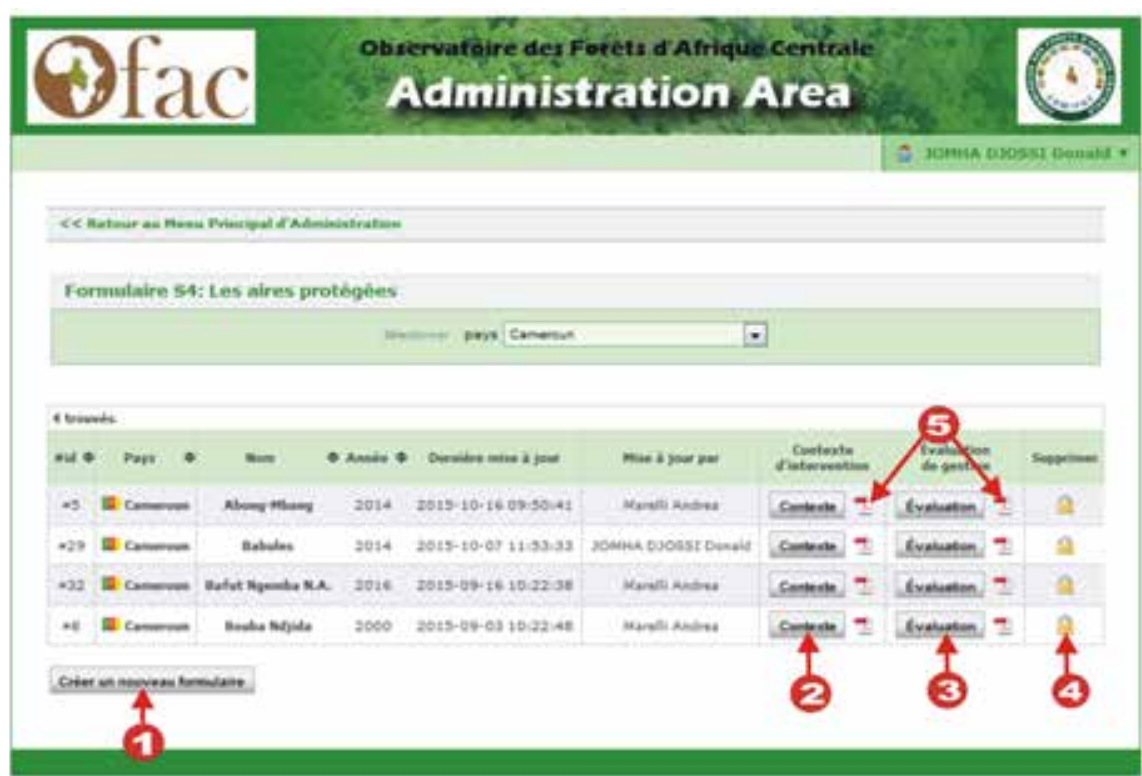

Schéma 16 - Liste de formulaires créés par pays 


\subsubsection{Opérations possibles dans le Formulaire}

À ce niveau, cinq types d'opérations sont envisageables :

1. Création d'un nouveau formulaire : En cliquant sur le bouton "Créer un nouveau formulaire " (cf. Schéma 17), I'utilisateur initie la création d'un nouveau formulaire. Le même formulaire ne peut pas être créé plusieurs fois ; en d'autres termes le système n'admet la création que d'un seul formulaire par aire protégée par année.

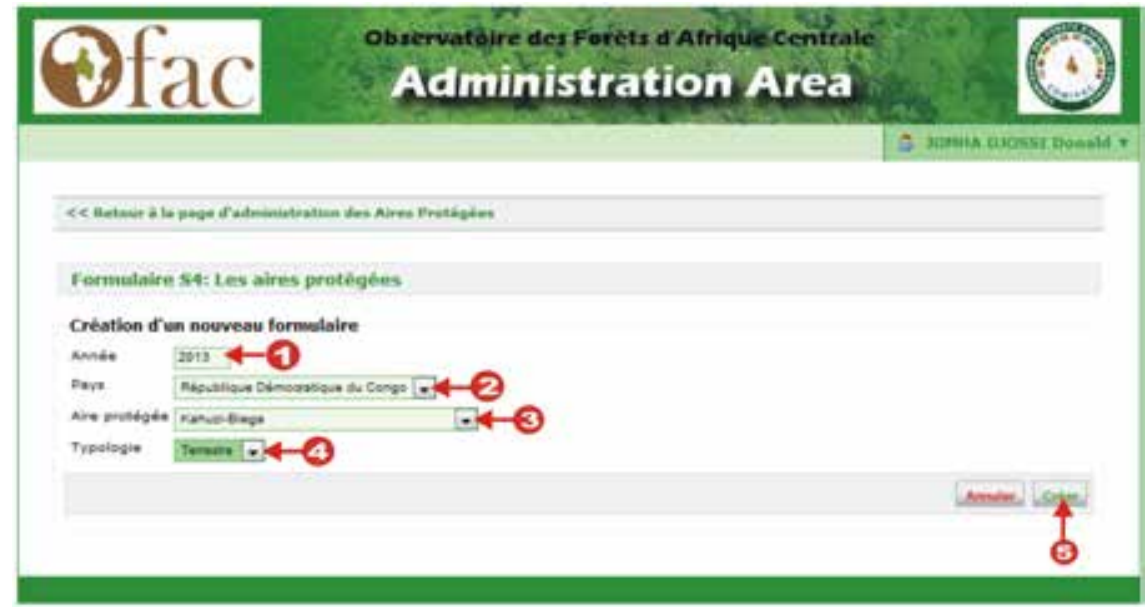

Schéma 17 - Création d'un formulaire

La création d'un nouveau formulaire demande de renseigner :

- l'année de campagne de la collecte de données (cf. Schéma 17, [1]);

- le pays où se trouve l'aire protégée (cf. Schéma 17, [2]) ;

- le nom de l'aire protégée (cette liste apparaît en fonction du pays choisi, seules les AP du pays choisi seront listées) (cf. Schéma 17, [3]) ;

- et le type d'aire protégée (cf. Schéma 17, [4]).

Une fois ces informations fournies, cliquez sur le bouton "Créer » (cf. Schéma 17, [5]). Cette opération vous dirige directement vers la première page du formulaire sur le contexte d'intervention avec le titre "Informations générales » où l'encodage proprement dit commence.

2. Encodage ou modification des données dans le formulaire du Contexte de l'intervention : En cliquant sur le bouton " Contexte " (cf. Schéma 16, [2]) l'utilisateur a la possibilité de voir toute les données du contexte d'intervention déjà encodées, de les modifier ou de les compléter pour le formulaire choisi. L'absence de ce bouton indique que l'utilisateur n'est pas concerné par l'AP.

3. Encodage ou modification des données dans le formulaire d'Evaluation de la gestion : En cliquant sur le bouton « Évaluation » (cf. Schéma 16, [3]) I'utilisateur à la possibilité de voir toutes les données et l'analyse graphique de l'Évaluation de la gestion déjà encodées, de les modifier ou de les compléter. L'absence de ce bouton indique que l'utilisateur n'est pas concerné par l'AP.

4. Suppression de formulaire : En cliquant sur l'icône (1-6) l'utilisateur supprime entièrement un formulaire, y compris ses données.

5. Extraction de données sous format PDF : En cliquant sur l'icône (프) (cf. Schéma 16, [5]) l'utilisateur génère le formulaire d'une aire protégée y compris ses données en format .pdf. II peut s'agir bien évidemment du formulaire relatif au Contexte de l'intervention ou à l'Évaluation de la gestion.

Note : bien que la liste complète des formulaires soit visible, l'utilisateur n'a la possibilité d'agir que sur certains formulaires et pour des actions dont il a les droits, d'où la présence éventuelle de l'icône cadenas (?) (cf. Schéma 16, [4]) indiquant " non autorisé ". 


\subsubsection{Structure générale du Formulaire : page et modules}

- Le Formulaire est organisé sous forme de pages, elles-mêmes constituées de modules. Un module est un ensemble de champs associés représentés dans un cadre. Chaque module est indépendant l'un de l'autre. Une barre supérieure (cf. Schéma 18) permet de naviguer entre les pages.

- L'intitulé de la page active est sur un fond vert foncé (cf. Schéma 18, [1]) contrairement à d'autres pages non actives.

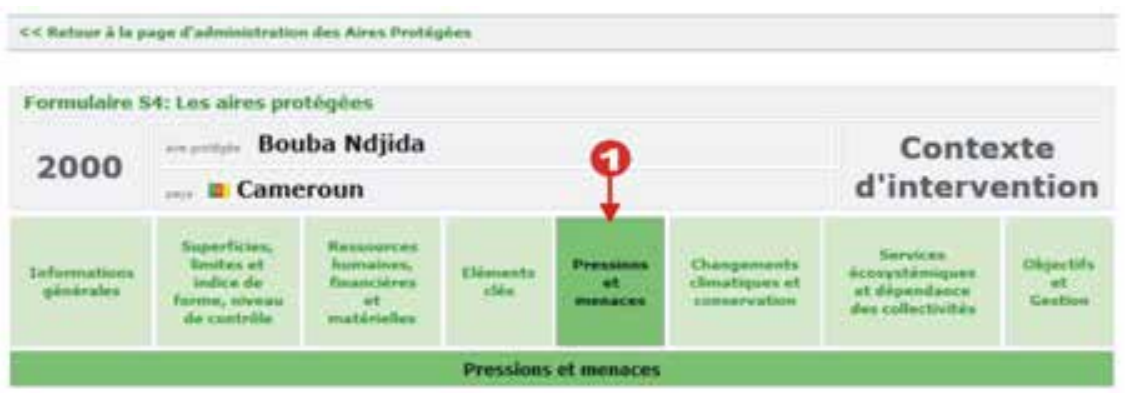

Schéma 18 - Barre de sélection de pages

- Dans chaque page, il existe plusieurs modules. Un module est un ensemble de champs associés présenté dans un cadre. De façon générale, les modules sont constitués de 2 grandes parties :

1. Les orientations et les indications. II est fortement conseillé de bien lire cette partie avant de passer à la deuxième qui est l'encodage proprement dit. II s'agit notamment de :

- L'indicateur : qui présente singulièrement l'ensemble de l'information sollicitée par le module (cf. Schéma 19, [1]).

- Méthodologie : guide l'utilisateur à clairement comprendre ce qui est demandé, ceci étant généralement exprimé sous forme de question (cf. Schéma 19, [2]).

- Critères - Concept mesuré - Variables : oriente les informations fournies par l'utilisateur à l'essentiel, et permet dans certains cas de garantir une cohérence avec les autres indicateurs déjà renseignés (cf. Schéma 19, [3]).

- Barème : précise littéralement le niveau affecté à chaque valeur de l'échelle (cf. Schéma 19, [4]).

2. L'ensemble des champs à remplir (cf. Schéma 19). Cette partie comprend des éléments tels que :

- des zones de texte : pour des saisies de textes ;

- des listes déroulantes : pour des insertions à choix unique ;

- des listes déroulantes à choix multiple : pour des insertions à plusieurs choix ;

- des cases à cocher : pour des choix avec des possibilités multiples ;

- des barres d'échelle : pour estimer le degré ;

- des boutons charger : pour charger des fichiers.

Note : Chaque module est indépendant et par conséquent enregistré individuellement.

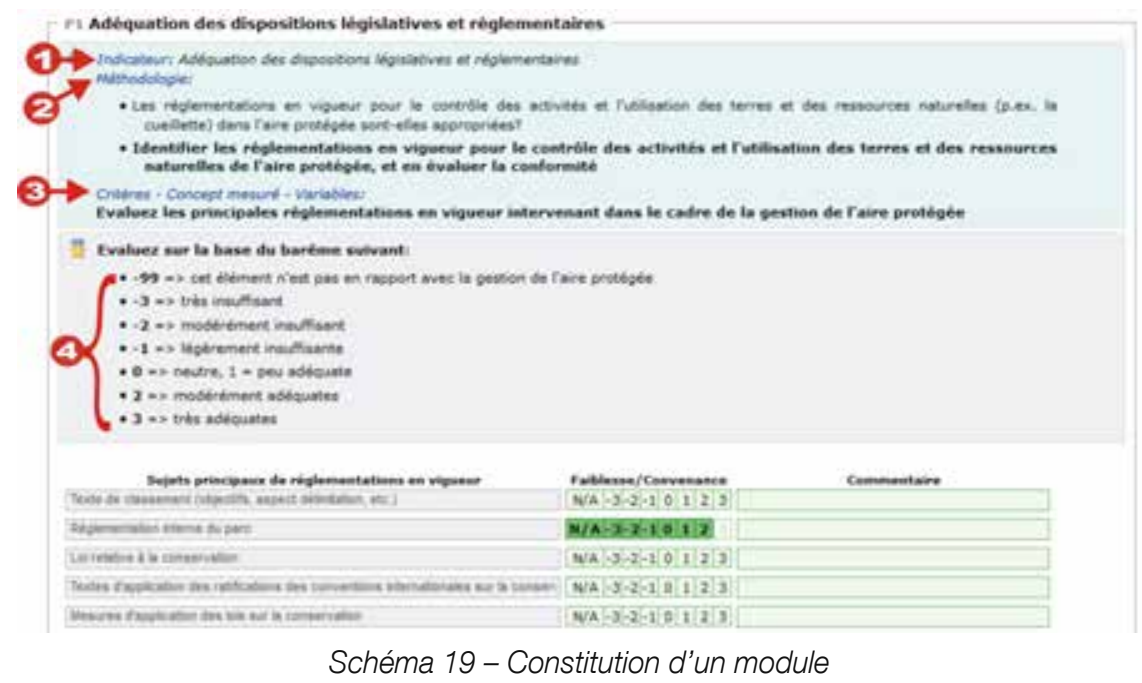




\subsubsection{Autres spécificités de l'encodage}

\section{Boite de dialogue Invite de saisie}

Pour certains champs, l'utilisateur est appelé à choisir à partir d'une recherche initiale l'élément à insérer dans le champ en question. Ceci s'applique par exemple à tous les champs où il est demandé de remplir une espèce : par exemple les champs de la page «Éléments clés » dans la partie "Contexte de la gestion ».

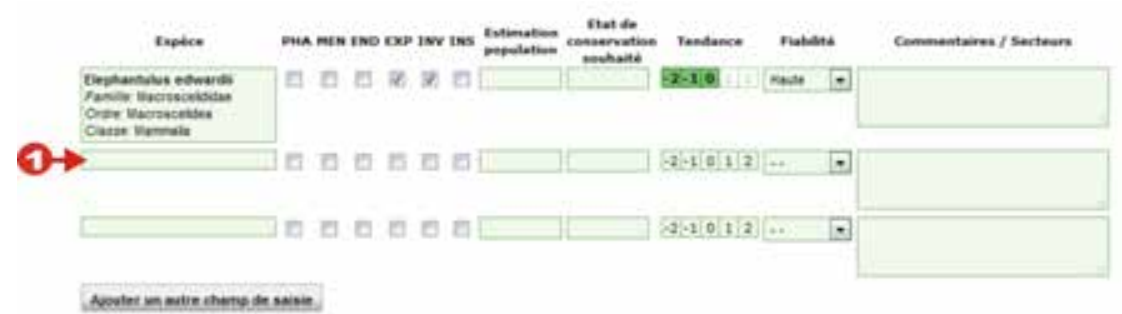

\section{Schéma 20 - Insertion d'éléments}

- Cliquez sur le champ comme pour insérer le curseur pour une saisie de texte (cf. Schéma 21, [1]).

- La boîte de dialogue d'Invite de recherche apparait (cf. Schéma 21).

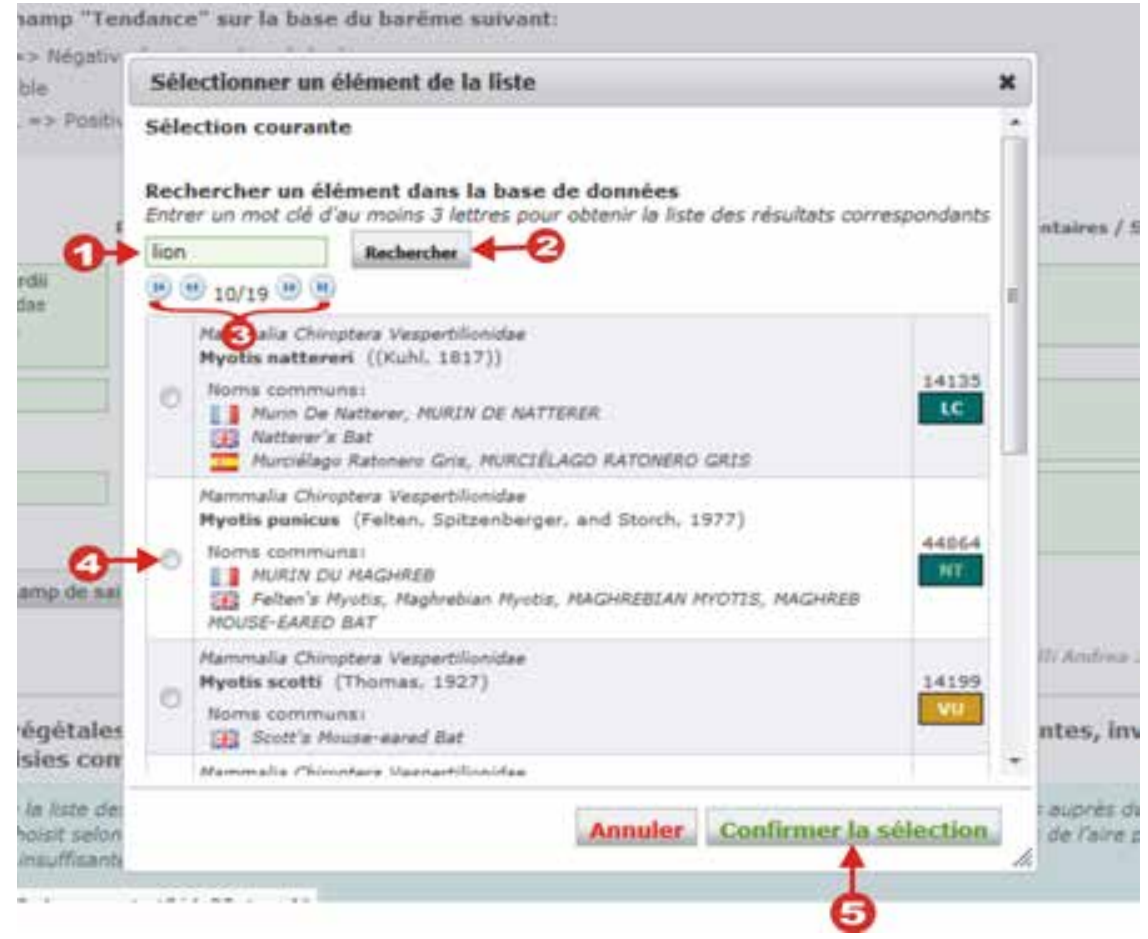

Schéma 21 - Boite de dialogue d'invite de recherche

Les numéros sur fond rouge donnent les étapes à suivre pour insérer un élément :

- Saisir un mot clé (saisir un seul mot sans espace, trois lettres minimum) (cf. Schéma 21, [1]) ;

- Exécuter la recherche (cf. Schéma 21, [2]) ;

- Naviguer entre les pages de la liste et Sélectionner l'élément recherché (cf. Schéma 21, [3]) ;

- Choisir l'élément à insérer (cf. Schéma 21, [4]) ;

- Confirmer votre sélection (cf. Schéma 21, [5]).

Note : Chaque module est indépendant et par conséquent enregistré individuellement. 


\section{Les menus « accordéon »}

Pour une meilleure lisibilité et un gain d'espace dans les pages du Formulaire, une série d'ensemble de champs identiques sont regroupés en panneaux que l'utilisateur peut ouvrir et fermer par un jeu de clicks (cf. Schéma 22, [1]), avec dans la majorité des cas la possibilité d'en rajouter.

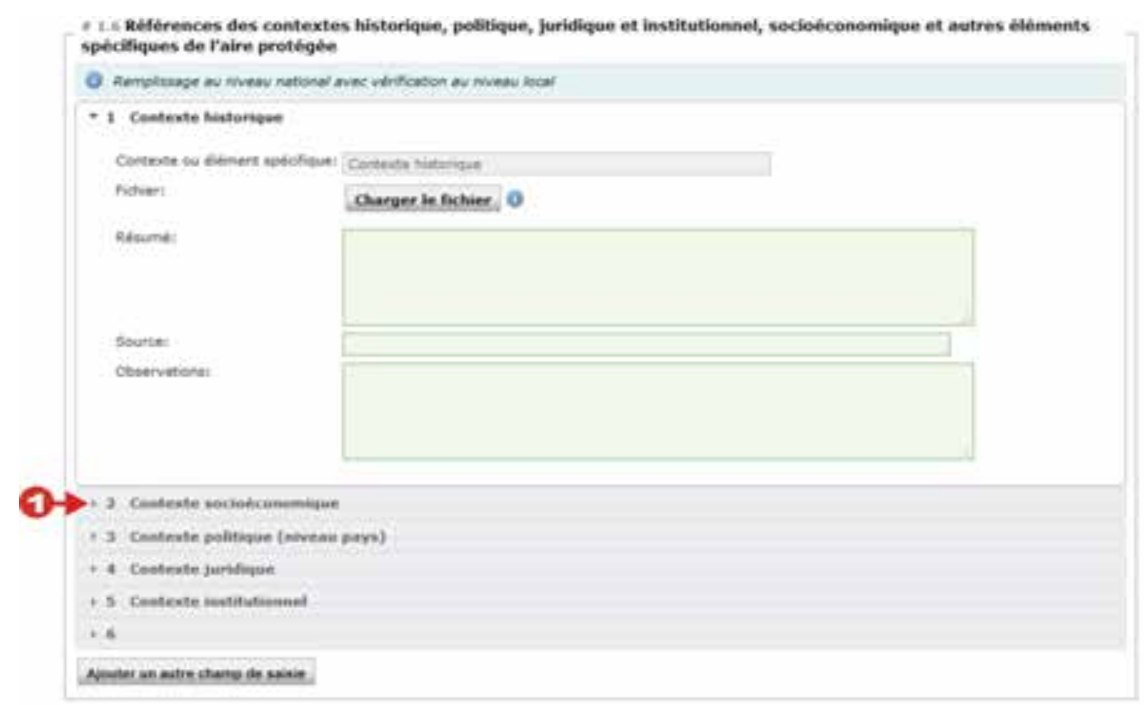

Schéma 22 - Les menus accordéon

\section{Les barèmes}

II est possible, après avoir choisi une valeur sur la barre de barème, que l'utilisateur veuille annuler son choix et réinitialiser la barre. Pour ce faire, double-cliquer sur la valeur la plus faible ou sur « N/A (Non Applicable)» (cf. Schéma 23, [1]).

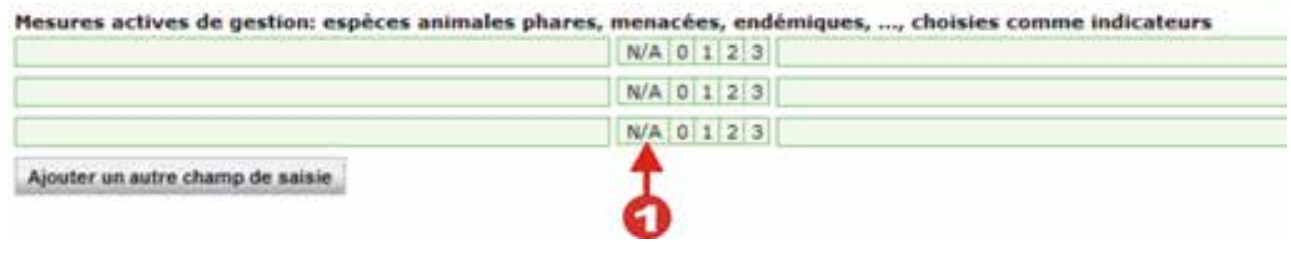

Schéma 23 - Réinitialisation de barème

\subsection{Créér et gérer son compte d'utilisateur}

\subsubsection{Comment créer un compte ?}

- Les étapes de création d'un compte commencent au niveau de la page de connexion, en cliquant sur le lien "Si vous n'avez pas encore de compte, créez-en un » (cf. Schéma 24, [1])

- Un ensemble d'informations est requis lors de la création d'un compte, d'ordre général et d'authentification (cf. Schéma 25)

- L'utilisateur a la possibilité de modifier ultérieurement les informations fournies, et de compléter les informations pertinentes à son profil.

Au terme de la création d'un compte, l'utilisateur a besoin des droits pour accéder au formulaire des aires protégées.

Note : N'oubliez pas le mot de passe que vous choisirez, car il sera impossible de le recouvrer. Toutefois vous pouvez le modifier quand vous le souhaitez. Retenez également que l'adresse e-mail entrée représente votre " Nom d'utilisateur ". 


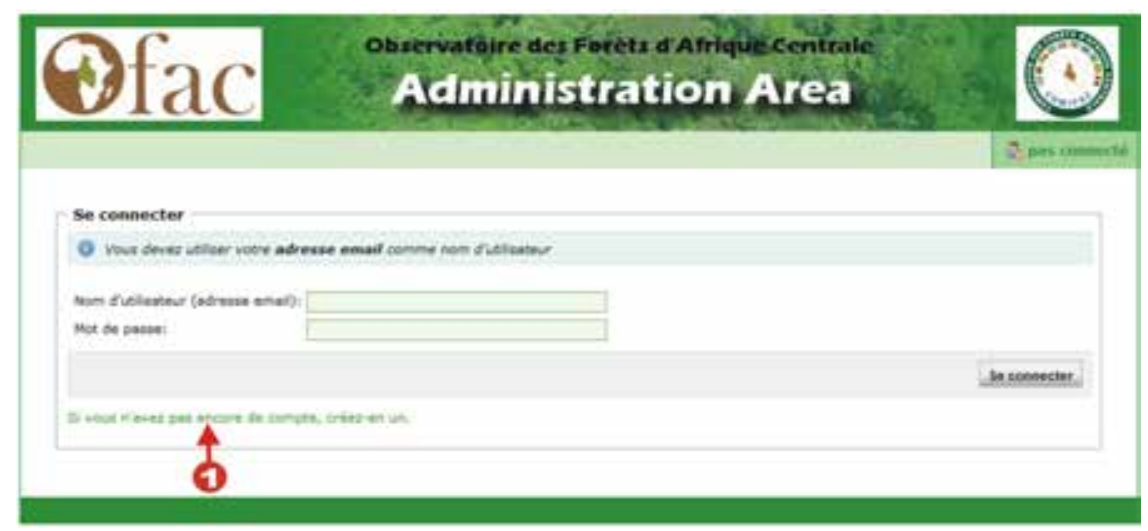

Schéma 24 - Page de connexion (Initier la création d'un compte)

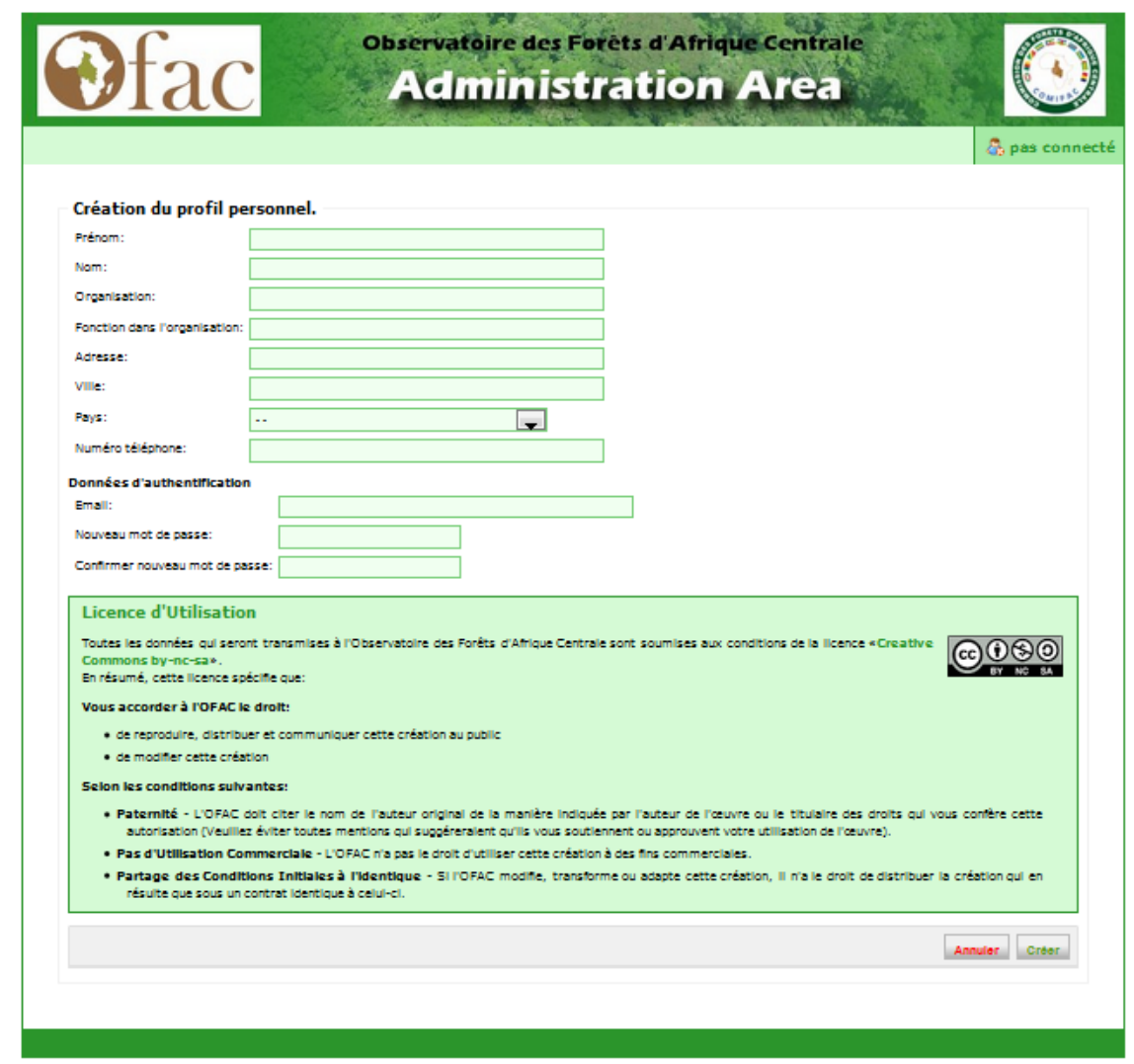

Schéma 25 - Création de compte d'utilisateur

\subsubsection{Comment gérer son profil d'utilisateur?}

Une fois connecté, l'utilisateur a la possibilité de modifier à tout moment les infor mations de son profil (adresse, email, téléphone, fonction, ...) et ses données d'authentification en cliquant sur le lien « Profil » (cf. Schéma 26, [1]) situé dans la partie supérieure droite de la page sous le menu intitulé par son nom.

\subsection{Sécurisation}

Étant donné que la version hors-ligne tourne sur des PC, les risques de pertes de données sont beaucoup plus nombreux qu'on ne le pense : simple panne de matériel due à la surtension électrique, vol, tasse de café qui se renverse, suppression par erreur, infection par un virus, malveillance d'un collaborateur ou encore catastrophes naturelles (incendies ou inondations)... Nous ne sommes jamais à l'abri d'une perte de données. C'est pour cela que nous ne pouvons pas nous contenter d'avoir ces données sur un seul et unique support numérique. 


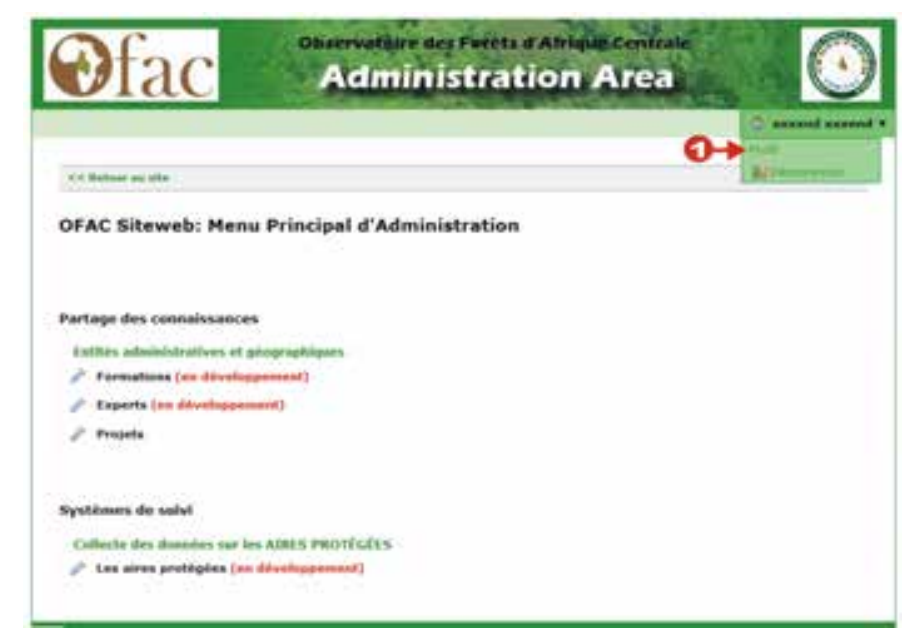

Schéma 26 - Menu d'administration (Modifier son profil)

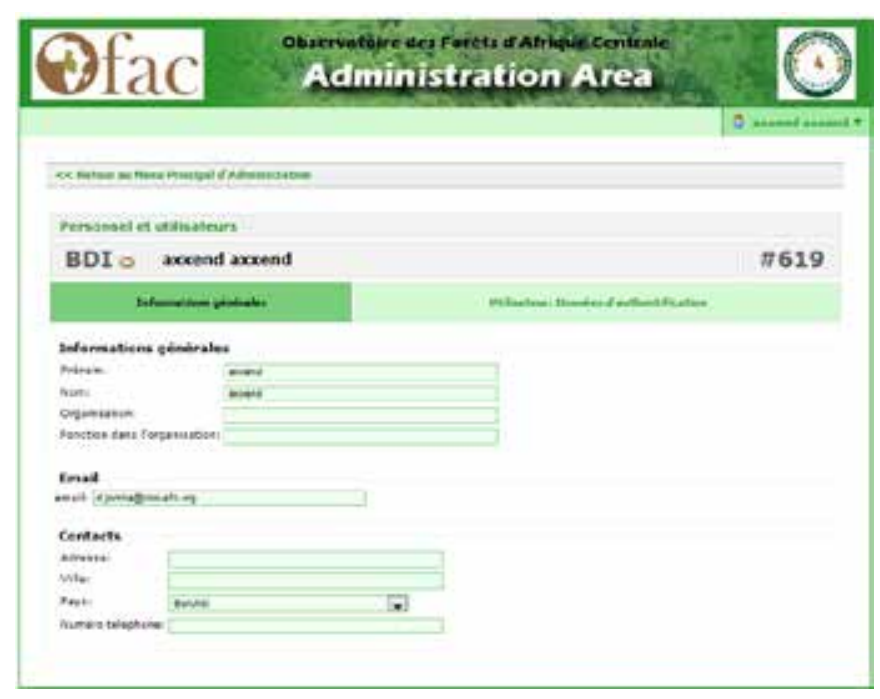

Schéma 27 - Modification des données de profil d'utilisateur

Cette section donne des astuces pour sécuriser vos données :

- Pour chaque aire protégée, il est fortement conseillé d'utiliser un seul ordinateur dans lequel fonctionnera l'application.

- Toutefois, face à la densité du Formulaire et aux délais de collecte de données, il peut arriver que les membres se partagent les parties pour la collecte de données. Même dans ce cas, la centralisation des données doit se faire sur un seul ordinateur.

- Après chaque séance de travail, il est important de faire une sauvegarde sur d'autres supports que la machine principale (disque dur externe, CD, clé USB, etc.). II faut alors copier tout le dossier contenant l'application.

- Après chaque séance de travail, n'oubliez pas de remplacer le dossier sur les supports de sauvegarde par le dossier actualisé.

\subsection{Ressources pour le coach}

En cas de difficultés

Carlos de Wasseige, OFAC

colewasseige@observatoire-comifac.net

Donald Jomha Djossi, OFAC

ddjossi@observatoire-comifac.net 


\section{PARTIE 5}

\section{Le Contrôle qualité}

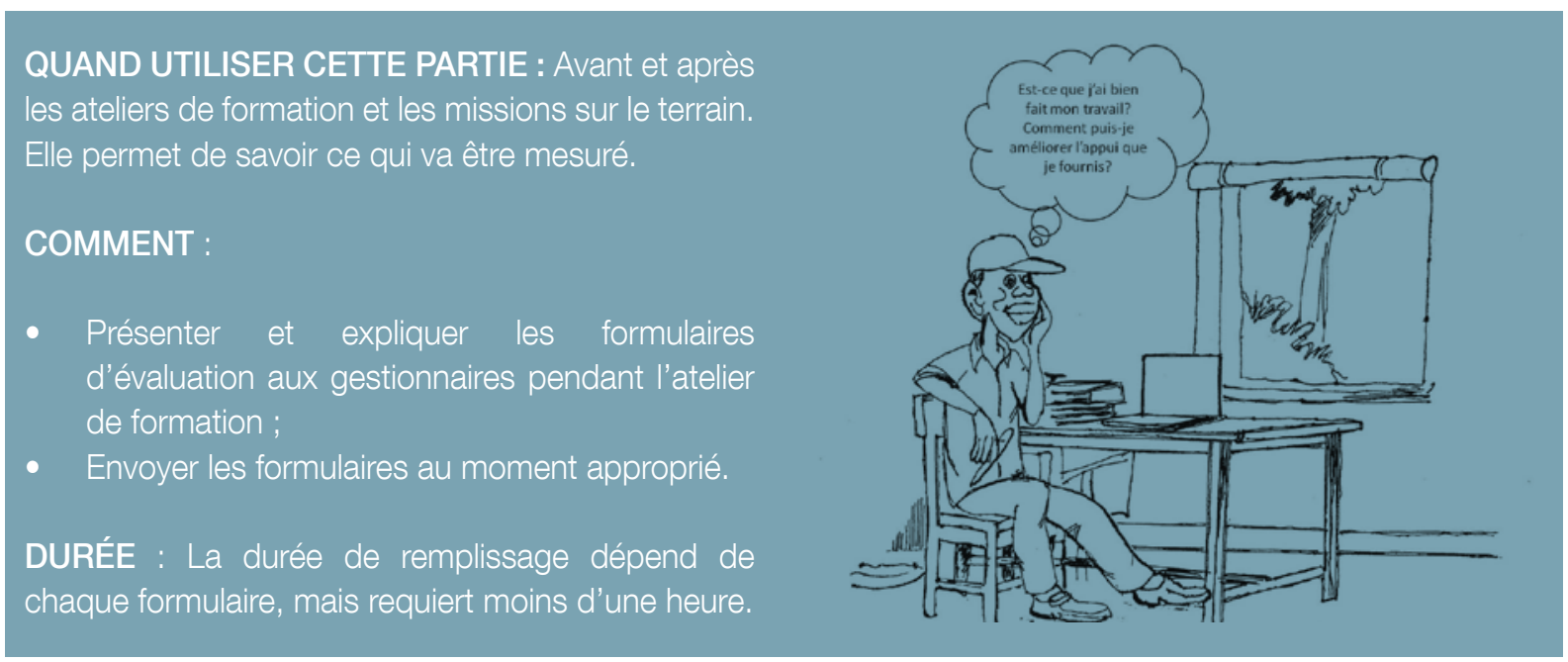

\subsection{Formulaires d'évaluation}

Cette partie comporte les outils d'évaluation qui visent à mesurer la qualité et l'efficacité des interventions des coaches, notamment en termes de renforcement des capacités des gestionnaires. Les résultats permettront de contribuer à l'évaluation générale du système d'information et de la phase I du Programme BIOPAMA en Afrique centrale et occidentale.

Pour les participants à la campagne de renforcement des capacités, l'évaluation fait partie du processus d'apprentissage. Elle aide le participant à mieux cerner ce qu'il a appris, à se connaître davantage et aussi à terminer un cycle d'apprentissage en formulant des recommandations qu'il souhaite pour le futur.

Trois principaux outils d'évaluation sont proposés aux coaches p our évaluer les parties prenantes dans le coaching.

Quelques astuces pour bien utiliser les formulaires d'évaluation :

- Le formulaire d'évaluation n'a rien de secret. Il est même important d'informer au préalable les personnes qui vont les remplir. Le formulaire peut être partagé même avant son utilisation pour expliquer son objectif.

- Si le formulaire est utilisé en présentiel : lire ensemble les différentes rubriques pour s'assurer que tout le monde comprend bien les questions et sait comment le remplir. Vous pouvez donner des exemples concrets.

- Tout le monde doit le remplir en même temps, pendant une durée déterminée.

- II faut s'assurer que les formulaires puissent être collectés en préservant l'anonymat.

Les formulaires d'évaluation présentés ci-après sont disponibles en fichiers distincts dans la clé USB de la COMIT. Les résultats de ces formulaires sont à rapporter dans les rapports à soumettre. 


\begin{tabular}{|l|l|l|l|}
\hline \multicolumn{2}{|l|}{ Outil d'évaluation 1} & C-EVAL1 & Formulaire d'évaluation des compétences de gestion d'une AP \\
\hline & Cibles de l'évaluation & Le conservateur d'une AP. \\
\hline & Évaluateur & $\begin{array}{l}\text { Le coach en utilisant la norme internationale de compétences en } \\
\text { gestion des aires protégées (CMAP/UICN). }\end{array}$ \\
\hline Objectifs de l'évaluation & $\begin{array}{l}\text { - Évaluer les compétences de gestion des Conservateurs selon des } \\
\text { normes internationales; } \\
\text { - Elvaluer les gains de compétences ; } \\
\text { Obtenir des orientations sur les efforts futurs de renforcement des } \\
\text { capacités. }\end{array}$ \\
\hline & Quand & Avant la formation et après la mission de coaching. \\
\hline Durée & $30-45$ min. \\
\hline Comment & À remplir à la main ou sous format électronique. \\
\hline
\end{tabular}

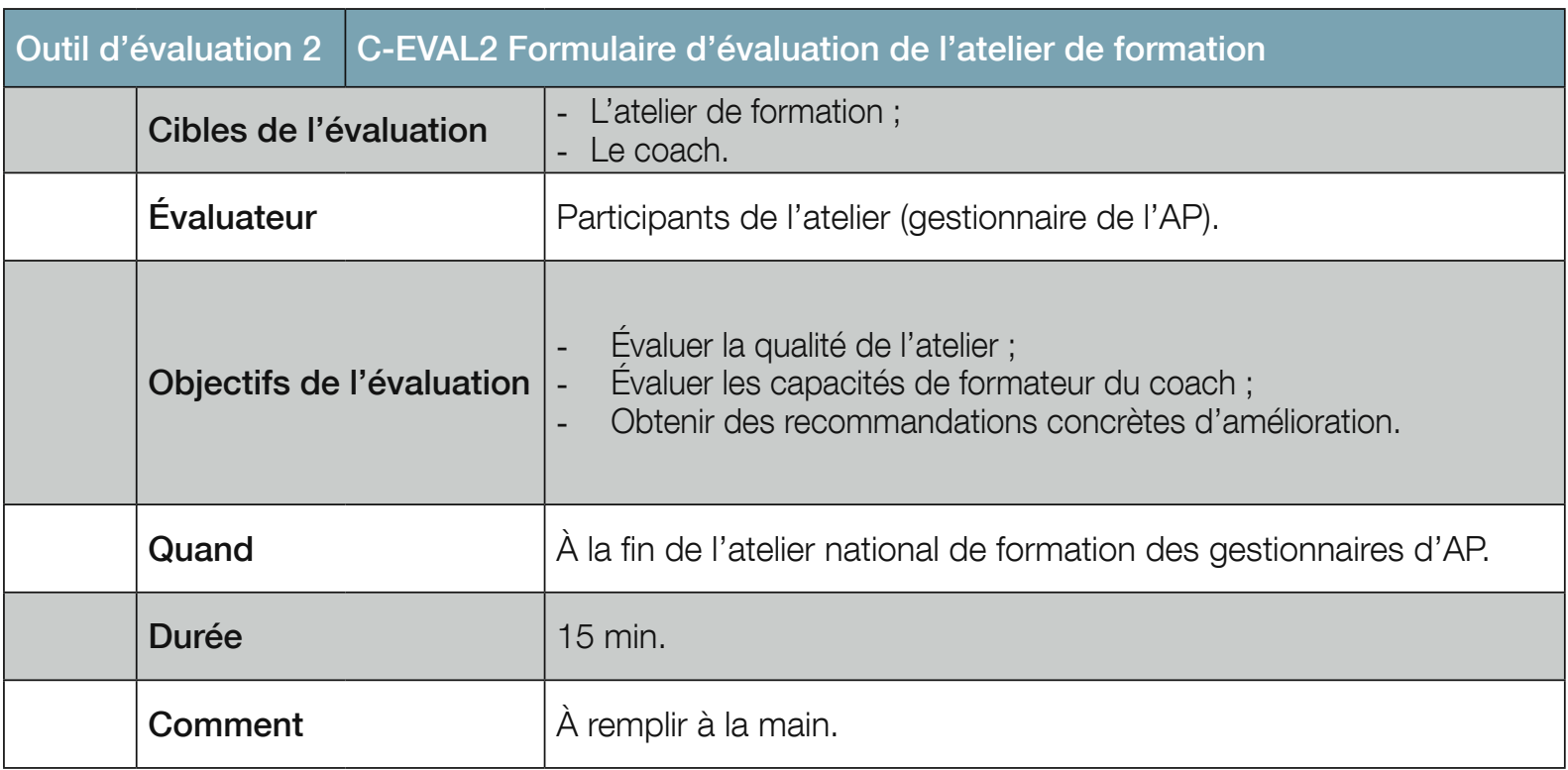

\begin{tabular}{|c|c|}
\hline Évaluation 3 & C-EVAL3 Formulaire d'évaluation de la mission de coaching \\
\hline Cibles de l'évaluation & $\begin{array}{ll}\text { - } & \text { Le coach ; } \\
\text { - } & \text { Les missions ; } \\
\text { - } & \text { Le gestionnaire. }\end{array}$ \\
\hline Évaluateur & Le conservateur et/ou l'équipe de gestion ; Institution nationale \\
\hline Objectifs de l'évaluation & $\begin{array}{l}\text { - Évaluer la qualité du coaching; } \\
\text { - } \quad \text { Mesurer les gains de compétences des gestionnaires ; } \\
\text { - Mesurer les capacités d'utilisation du Formulaire et du système } \\
\text { d'information par les gestionnaires; } \\
\text { - Mesurer les transformations institutionnelles dans le cadre de } \\
\text { l'utilisation du Système d'information; } \\
\text { - Obtenir des indications claires pour l'amélioration future de } \\
\text { l'approche de coaching. }\end{array}$ \\
\hline Quand & $\begin{array}{l}\text { À la fin de l'atelier national de formation des gestionnaires d'AP. À } \\
\text { intégrer dans le rapport technique. }\end{array}$ \\
\hline Durée & $30 \mathrm{~min}$. \\
\hline Comment & À remplir sous format électronique. \\
\hline
\end{tabular}


FORMULAIRE D'ÉVALUATION DES

COMPÉTENCES DE GESTION D'UNE AP

AUTO-ÉVALUATION DES COMPÉTENCES DANS LES AIRES PROTÉGÉES - Informations générales

\begin{tabular}{|c|c|c|c|c|c|}
\hline \multicolumn{6}{|l|}{ PAYS } \\
\hline \multicolumn{6}{|l|}{$\begin{array}{l}\text { NOM. Prénom } \\
\text { Écrivez clairement svp }\end{array}$} \\
\hline SEXE & M & $F$ & & & \\
\hline AGE (Entourez une réponse) & $<30$ & $31-45$ & $46-60$ & $>60$ & \\
\hline \multicolumn{6}{|l|}{$\begin{array}{l}\text { TITRE OFFICIEL DE L'EMPLOI } \\
\text { (Grade) }\end{array}$} \\
\hline \multicolumn{6}{|l|}{ EMPLOYEUR } \\
\hline \multicolumn{6}{|l|}{ LIEU DE TRAVAIL } \\
\hline $\begin{array}{l}\text { ANNÉES D'EXPÉRIENCE } \\
\text { PERTINENTE } \\
\text { Entourez une réponse }\end{array}$ & $0-5$ & $6-10$ & $11-15$ & $16+$ & \\
\hline $\begin{array}{l}\text { NIVEAU D'ÉTUDES LE PLUS } \\
\text { ÉLEVÉ } \\
\text { Entourez une réponse }\end{array}$ & $\begin{array}{c}\text { École } \\
\text { élémentaire }\end{array}$ & Lycée & Licence & Master & Doctorat \\
\hline
\end{tabular}

FORMATIONS LIÉES AU TRAVAIL DURANT LES 3 DERNIERES ANNÉES

\begin{tabular}{|l|l|l|l|}
\hline \multirow{2}{*}{ THÈME } & \multirow{2}{*}{ Fournisseur } & \multicolumn{2}{|c|}{ Durée } \\
\cline { 3 - 5 } & & Mois & Jours \\
\hline & & & \\
\hline & & & \\
\hline & & & \\
\hline & & & \\
\hline
\end{tabular}

A COMPLÉTER PAR LE SUPERVISEUR DE L'ÉVALUATION

\begin{tabular}{|c|c|c|c|c|c|}
\hline NIVEAU GÉNÉRAL & (1) & (2) & (3) & (4) & \\
\hline \multirow{3}{*}{ CATÉGORIES ÉVALUÉES } & PPP & ORG & HRM & ADR & $\mathrm{BIO}$ \\
\hline & LAR & COM & TRP & AWA & FLD \\
\hline & TEC & CAC & UNI & & \\
\hline \multicolumn{6}{|l|}{ NOM DE L'ÉVALUATEUR } \\
\hline \multicolumn{6}{|l|}{$\begin{array}{l}\text { DATE DE L'ÉVALUATION } \\
\text { (D M A) }\end{array}$} \\
\hline \multicolumn{6}{|l|}{ LIEU DE L'ÉVALUATION } \\
\hline Code d'évaluation & & & & & \\
\hline
\end{tabular}




\section{AUTO-ÉVALUATION DES COMPÉTENCES DANS LES AIRES PROTÉGÉES. NIVEAU 3.}

\begin{tabular}{|l|l|}
\hline \multicolumn{1}{|c|}{ NOM } & \\
\hline \multicolumn{1}{|c|}{ CODE } & \\
\hline X- Cette capacité n'est pas pertinente pour mon travail. \\
0 - Cette capacité est partiellement pertinente pour mon travail, mais je n'en ai pas régulièrement besoin. \\
$\mathbf{1}$ - Cette capacité est régulièrement requise dans mon travail, mais je n'ai pas ou peu cette compétence. \\
J'ai besoin de formation ou d'appuis importants à ce sujet. \\
2 - Cette capacité est régulièrement requise dans mon travail, et j'ai une légère compétence à ce sujet. \\
J'ai besoin de formation et d'appui. \\
3 - Cette capacité est régulièrement requise dans mon travail, et j'ai une bonne compétence à ce sujet. \\
J'ai seulement besoin d'actualisation/de recyclage périodique. \\
4 - Cette capacité est régulièrement requise dans mon travail et j'ai une haute compétence à ce sujet. Je \\
pourrais former d'autres personnes sur cette capacité.
\end{tabular}

\begin{tabular}{|c|c|c|}
\hline GROUPE & PLANIFICATION, GESTION ET ADMINISTRATION & \\
\hline CATEGORIE & PPP. POLITIQUES, PLANIFICATION ET PROJETS LIÉS AUX AP & \multirow{3}{*}{$\begin{array}{c}\times 012 \\
34\end{array}$} \\
\hline PPP 3 & POLITIQUES, PLANIFICATION ET PROJETS LIÉS AUX AP. NIVEAU 3 & \\
\hline Code & Compétences. Si nécessaire, l'individu devra être capable de : & \\
\hline PPP 3.1 & $\begin{array}{l}\text { Diriger le développement participatif du plan de gestion d'une aire protégée en } \\
\text { utilisant les formats et processus reconnus. }\end{array}$ & \\
\hline PPP 3.2 & Diriger une évaluation structurée des menaces pour une aire protégée. & \\
\hline PPP 3.3 & Diriger l'élaboration d'un système de zonage d'une aire protégée. & \\
\hline PPP 3.4 & $\begin{array}{l}\text { Diriger l'élaboration de propositions et de plans de projets pour une aire } \\
\text { protégée en utilisant les formats et les processus reconnus. }\end{array}$ & \\
\hline PPP 3.5 & Diriger la mise en œuvre de plans et de projets. & \\
\hline PPP 3.6 & $\begin{array}{l}\text { Diriger la réalisation d'évaluations des risques/désastres et des plans } \\
\text { d'urgence. }\end{array}$ & \\
\hline PPP 3.7 & $\begin{array}{l}\text { Diriger l'identification et la mise en œuvre des mesures pour faire face aux } \\
\text { impacts du changement climatique. }\end{array}$ & \\
\hline PPP 3.8 & $\begin{array}{l}\text { Superviser la planification, la mise en œuvre et le suivi des projets } \\
\text { d'infrastructure. }\end{array}$ & \\
\hline PPP 3.9 & $\begin{array}{l}\text { Coordonner la gestion de l'aire protégée avec les activités des propriétaires et } \\
\text { des utilisateurs des terres et ressources environnantes. }\end{array}$ & \\
\hline PPP 3.10 & $\begin{array}{l}\text { Contribuer aux Études d'impacts environnementaux (EIE) des projets et des } \\
\text { propositions affectant l'aire protégée. }\end{array}$ & \\
\hline
\end{tabular}

\begin{tabular}{|c|c|c|}
\hline CATEGORY & ORG. LEADERSHIP ET DÉVELOPPEMENT ORGANISATIONNEL & \multirow{3}{*}{$\begin{array}{c}\times 012 \\
34\end{array}$} \\
\hline ORG 3 & LEADERSHIP ET DÉVELOPPEMENT ORGANISATIONNEL. NIVEAU 3 & \\
\hline Code & Compétence. Si nécessaire, l'individu devra être capable de : & \\
\hline ORG 3.1 & $\begin{array}{l}\text { Assurer les progrès envers l'acquisition des ressources humaines, physiques et } \\
\text { financières adéquates pour la gestion effective de l'aire protégée. }\end{array}$ & \\
\hline ORG 3.2 & $\begin{array}{l}\text { Établir des procédures pour une gestion structurée, planifiée et adaptative de } \\
\text { l'aire protégée. }\end{array}$ & \\
\hline ORG 3.3 & Établir une planification et un suivi systématique de la gestion des activités. & \\
\hline ORG 3.4 & $\begin{array}{l}\text { Établir des systèmes et des procédures pour assurer des normes élevées } \\
\text { d'éthique et de comportement envers le personnel et les partenaires. }\end{array}$ & \\
\hline
\end{tabular}




\begin{tabular}{|c|l|l|}
\hline ORG 3.5 & $\begin{array}{l}\text { Construire des réseaux et développer des relations collaboratives avec les } \\
\text { autres organisations. }\end{array}$ & \\
\hline ORG 3.6 & Assurer la mise en place et l'application d'une gouvernance participative. & \\
\hline ORG 3.7 & $\begin{array}{l}\text { Établir des systèmes et des procédures pour assurer la santé, la sûreté et la } \\
\text { sécurité dans une aire protégée. }\end{array}$ & \\
\hline ORG 3.8 & $\begin{array}{l}\text { Promouvoir et mettre en place le changement et l'innovation dans la gestion } \\
\text { d'une aire protégée. }\end{array}$ & \\
\hline ORG 3.9 & $\begin{array}{l}\text { Mettre en œeuvre des processus pour des consultations et des prises de } \\
\text { décisions inclusives, transparentes et participatives. }\end{array}$ & \\
\hline ORG 3.10 & Assurer la gestion efficace de l'information et de la connaissance. & \\
\hline ORG 3.11 & $\begin{array}{l}\text { Sécuriser les reconnaissances certifiées de la qualité de la gestion d'une aire } \\
\text { protégée. }\end{array}$ & \\
\hline
\end{tabular}

\begin{tabular}{|c|c|c|}
\hline CATEGORY & ADR. DOCUMENTATION ET NOTIFICATION ADMINISTRATIVE & \multirow{3}{*}{$\begin{array}{c}\times 012 \\
34\end{array}$} \\
\hline ADR 3 & DOCUMENTATION ET NOTIFICATION ADMINISTRATIVE. NIVEAU 3 & \\
\hline Code & Compétence. Si nécessaire, l'individu devra être capable de : & \\
\hline ADR 3.1 & $\begin{array}{l}\text { Compiler et préparer les rapports majeurs sur l'aire protégée et sur une activité } \\
\text { de projet. }\end{array}$ & \\
\hline ADR 3.2 & $\begin{array}{l}\text { Organiser, gérer et assurer la documentation dans les réunions, les } \\
\text { consultations et les négociations. }\end{array}$ & \\
\hline ADR 3.3 & $\begin{array}{l}\text { Garantir que les archives et les documentations complètes sont maintenues et } \\
\text { sécurisées pour une aire protégée. }\end{array}$ & \\
\hline ADR 3.4 & $\begin{array}{l}\text { Mettre en œuvre des mesures pour le suivi et la notification exhaustifs sur la } \\
\text { performance de l'aire protégée. }\end{array}$ & \\
\hline
\end{tabular}

\begin{tabular}{|c|l|c|}
\hline GROUPE & \multicolumn{1}{|c|}{ PERMETTRE LES COMPÉTENCES PERSONNELLES } & \\
\hline CATEGORY & CAC. COMMUNICATION ET COLLABORATION & X 012 \\
$\mathbf{3} 4$ \\
\hline CAC 3 & COMMUNICATION ET COLLABORATION. NIVEAU 3 & \\
\hline Code & Compétence. Si nécessaire, l'individu devra être capable de : & \\
\hline CAC 3.1 & Maintenir une communication efficace au sein de l'organisation. & \\
\hline CAC 3.2 & $\begin{array}{l}\text { Maintenir une communication et de bonnes relations de travail avec les parties } \\
\text { prenantes et les partenaires. }\end{array}$ & \\
\hline CAC 3.3 & Négocier des accords et résoudre les différends et les conflits. & \\
\hline CAC 3.4 & $\begin{array}{l}\text { Garantir des consultations et des prises de décisions transparentes et } \\
\text { participatives. }\end{array}$ & \\
\hline
\end{tabular}

Nous vous remercions d'avoir pris le temps de remplir ce formulaire.

Traduction non officielle. Utilisé pour le programme BIOPAMA Afrique centrale et occidentale avec l'aimable autorisation de Appleton, M.R. (2015) Global Register of Protected Area Competences. Version provisoire. Disponible auprès de : protectedareacompetences@gmail.com 
C-EVAL 2

FICHE D'ÉVALUATION DE L'ATELIER NATIONAL DE FORMATION DES GESTIONNAIRES D'AIRES PROTÉGÉES ET DES ACTEURS DE LA CONSERVATION
Nous vous remercions de bien vouloir contribuer à l'évaluation de l'atelier afin d'améliorer les futures activités. Veuillez entourer les réponses qui correspondent à votre choix.

À remplir à la fin de l'atelier de formation.

Modifiable selon les objectifs fixés

Dates de l'atelier

Lieu (ville, pays)

\begin{tabular}{|l|c|c|c|c|c|}
\hline 1. En général & & & & & \\
\hline $\begin{array}{l}\text { Quelle note donneriez-vous à la qualité } \\
\text { générale de la formation? }\end{array}$ & $\begin{array}{c}\text { Très faible } \\
1\end{array}$ & $\begin{array}{c}\text { Faible } \\
2\end{array}$ & $\begin{array}{c}\text { Moyenne } \\
3\end{array}$ & $\begin{array}{c}\text { Bonne } \\
4\end{array}$ & $\begin{array}{c}\text { Excellente } \\
5\end{array}$ \\
\hline $\begin{array}{l}\text { Quel pourcentage des sujets présentés } \\
\text { et discutés avez-vous compris ? }\end{array}$ & $0-20 \%$ & $20-40 \%$ & $40-60 \%$ & $60-80 \%$ & $80-100 \%$ \\
\hline
\end{tabular}

\begin{tabular}{|l|c|c|c|c|c|}
\hline 2. Contenu de l'atelier & \multicolumn{2}{|c|}{ Pas du tout d'accord } & & \multicolumn{2}{c|}{ Tout à fait d'accord } \\
\hline Les objectifs de l'atelier étaient clairs & 1 & 2 & 3 & 4 & 5 \\
\hline $\begin{array}{l}\text { Les thèmes présentés étaient cohérents } \\
\text { par rapport aux objectifs }\end{array}$ & 1 & 2 & 3 & 4 & 5 \\
\hline $\begin{array}{l}\text { Le contenu était présenté et organisé } \\
\text { dans une suite logique }\end{array}$ & 1 & 2 & 3 & 4 & 5 \\
\hline $\begin{array}{l}\text { Les activités pratiques (exercices) } \\
\text { de l'atelier ont permis d'atteindre les } \\
\text { objectifs }\end{array}$ & 1 & 2 & 3 & 4 & 5 \\
\hline $\begin{array}{l}\text { L'atelier a constitué une bonne occasion } \\
\text { de se familiariser avec les coaches }\end{array}$ & 1 & 2 & 3 & 4 & 5 \\
\hline $\begin{array}{l}\text { L'atelier a constitué une bonne } \\
\text { occasion de créer des liens avec } \\
\text { d'autres participants }\end{array}$ & 1 & 2 & 3 & 4 & 5 \\
\hline
\end{tabular}

\begin{tabular}{|l|c|c|c|c|c|}
\hline 3. Organisation et présentation & $\begin{array}{c}\text { Très faible/ } \\
\text { Très } \\
\text { insuffisant }\end{array}$ & $\begin{array}{c}\text { Faible/ } \\
\text { Insuffisant }\end{array}$ & $\begin{array}{c}\text { Moyen/ } \\
\text { Neutre }\end{array}$ & $\begin{array}{c}\text { Bon/ } \\
\text { Satisfaisant }\end{array}$ & $\begin{array}{c}\text { Excellent/ } \\
\text { Très } \\
\text { satisfaisant }\end{array}$ \\
\hline L'endroit choisi était... & 1 & 2 & 3 & 4 & 5 \\
\hline L'organisation logistique était... & 1 & 2 & 3 & 4 & 5 \\
\hline Le nombre de participants était... & 1 & 2 & 3 & 4 & 5 \\
\hline La durée de l'atelier était... & 1 & 2 & 3 & 4 & 5 \\
\hline L'équilibre entre les activités était... & 1 & 2 & 3 & 4 & 5 \\
\hline $\begin{array}{l}\text { Le temps alloué à chaque activité } \\
\text { était... }\end{array}$ & 1 & 2 & 3 & 4 & 5 \\
\hline La diversité des activités était... & 1 & 2 & 3 & 4 & 5 \\
\hline $\begin{array}{l}\text { La productivité des travaux de groupe } \\
\text { et autres activités était... }\end{array}$ & 1 & 2 & 3 & 4 & 5 \\
\hline
\end{tabular}


4. ÉVALUATION DES SESSIONS

\begin{tabular}{|l|c|c|c|c|c|}
\hline Veuillez évaluer les différentes sessions & Très faible & Faible & Moyen & Bon & Excellent \\
\hline $\begin{array}{l}\text { SESSION 1 : Présentation générale } \\
\text { : BIOPAMA, Observatoire régional, } \\
\text { Système d'information }\end{array}$ & 1 & 2 & 3 & 4 & 5 \\
\hline $\begin{array}{l}\text { SESSION 2 : La gestion et la } \\
\text { gouvernance des aires protégées }\end{array}$ & 1 & 2 & 3 & 4 & 5 \\
\hline SESSION 3 : Explication du Formulaire & 1 & 2 & 3 & 4 & 5 \\
\hline $\begin{array}{l}\text { SESSION 4 : Exercices sur des } \\
\text { éléments du Formulaire : contexte de } \\
\text { l'intervention }\end{array}$ & 1 & 2 & 3 & 4 & 5 \\
\hline $\begin{array}{l}\text { SESSION 5 : Exercices sur des } \\
\text { éléments du Formulaire : efficacité de la } \\
\text { gestion }\end{array}$ & 1 & 2 & 3 & 4 & 5 \\
\hline $\begin{array}{l}\text { SESSION 6 : Étude de cas/Visite sur le } \\
\text { terrain }\end{array}$ & 1 & 2 & 3 & 4 & 5 \\
\hline $\begin{array}{l}\text { SESSION 7 : Préparation de l'étape de } \\
\text { terrain }\end{array}$ & 1 & 2 & 3 & 4 & 5 \\
\hline SESSION 8 : Analyse et restitution & 1 & 2 & 3 & 4 & 5 \\
\hline
\end{tabular}

5. ÉVALUATION DES COACHES FORMATEURS

Nom du Coach 1

\begin{tabular}{|l|c|c|c|c|c|}
\hline Le coach a..... & \multicolumn{2}{|c|}{ Pas du tout d'accord } & & \multicolumn{2}{|c|}{ Tout à fait d'accord } \\
\hline $\begin{array}{l}\text { Aidé à se familiariser avec le Système } \\
\text { d'information sur les aires protégées et } \\
\text { la biodiversité }\end{array}$ & 1 & 2 & 3 & 4 & 5 \\
\hline $\begin{array}{l}\text { Donné les instructions nécessaires pour } \\
\text { remplir correctement le Formulaire }\end{array}$ & 1 & 2 & 3 & 4 & 5 \\
\hline $\begin{array}{l}\text { Fourni des explications et des exemples } \\
\text { pertinents pour mieux comprendre le } \\
\text { Formulaire }\end{array}$ & 1 & 2 & 3 & 4 & 5 \\
\hline $\begin{array}{l}\text { Stimulé et favorisé l'esprit d'analyse des } \\
\text { participants }\end{array}$ & 1 & 2 & 3 & 4 & 5 \\
\hline
\end{tabular}

\begin{tabular}{|l|c|c|c|c|c|}
\hline Mis les participants en confiance & 1 & 2 & 3 & 4 & 5 \\
\hline $\begin{array}{l}\text { Géré efficacement les différentes } \\
\text { interventions des participants et les } \\
\text { discussions }\end{array}$ & 1 & 2 & 3 & 4 & 5 \\
\hline A facilité les progrès de l'atelier & 1 & 2 & 3 & 4 & 5 \\
\hline $\begin{array}{l}\text { A maintenu l'intérêt et la participation de } \\
\text { tous les participants }\end{array}$ & 1 & 2 & 3 & 4 & 5 \\
\hline $\begin{array}{l}\text { A bien aidé les participants qui } \\
\text { rencontraient des problèmes }\end{array}$ & 1 & 2 & 3 & 4 & 5 \\
\hline Était efficace et sympathique & 1 & 2 & 3 & 4 & 5 \\
\hline
\end{tabular}


Nom du Coach 2

\begin{tabular}{|l|c|c|c|c|c|}
\hline Le coach a.... & \multicolumn{2}{|c|}{ Pas du tout d'accord } & \multicolumn{2}{|c|}{ Tout à fait d'accord } \\
\hline $\begin{array}{l}\text { Aidé à se familiariser avec le Système } \\
\text { d'information sur les aires protégées et } \\
\text { la biodiversité }\end{array}$ & 1 & 2 & 3 & 4 & 5 \\
\hline $\begin{array}{l}\text { Donné les instructions nécessaires pour } \\
\text { remplir correctement le Formulaire }\end{array}$ & 1 & 2 & 3 & 4 & 5 \\
\hline $\begin{array}{l}\text { Fourni des explications et des exemples } \\
\text { pertinents pour mieux comprendre le } \\
\text { Formulaire }\end{array}$ & 1 & 2 & 3 & 4 & 5 \\
\hline $\begin{array}{l}\text { Stimulé et favorisé l'esprit d'analyse des } \\
\text { participants }\end{array}$ & 1 & 2 & 3 & 4 & 5 \\
\hline
\end{tabular}

\begin{tabular}{|l|c|c|c|c|c|}
\hline Mis les participants en confiance & 1 & 2 & 3 & 4 & 5 \\
\hline $\begin{array}{l}\text { Géré efficacement les différentes } \\
\text { interventions des participants et les } \\
\text { discussions }\end{array}$ & 1 & 2 & 3 & 4 & 5 \\
\hline A facilité les progrès de l'atelier & 1 & 2 & 3 & 4 & 5 \\
\hline $\begin{array}{l}\text { A maintenu l'intérêt et la participation de } \\
\text { tous les participants }\end{array}$ & 1 & 2 & 3 & 4 & 5 \\
\hline $\begin{array}{l}\text { A bien aidé les participants qui } \\
\text { rencontraient des problèmes }\end{array}$ & 1 & 2 & 3 & 4 & 5 \\
\hline Était efficace et sympathique & 1 & 2 & 3 & 4 & 5 \\
\hline
\end{tabular}

\section{QUESTIONS SUPPLÉMENTAIRES}

Pour vous, quelle a été la partie la plus importante ou la plus utile de cette formation?

Qu'auriez-vous aimé apprendre davantage lors de cette formation?

Avez-vous d'autres commentaires pour améliorer la formation dans le futur?

Nous vous remercions d'avoir pris le temps de remplir ce formulaire 
C-EVAL 3

\section{FICHE D'ÉVALUATION DE LA MISSION DE COACHING DES AIRES PROTÉGÉES ET ACTEURS DE LA CONSERVATION}

Nous vous remercions de bien vouloir contribuer à l'évaluation de l'atelier afin d'améliorer les futures activités. Veuillez choisir les réponses qui correspondent à votre choix.

\section{Pays concerné}

\section{En général}

Échelle : 1 : Très faible - 2 : Faible - 3 : Moyenne $-4:$ Bonne - 5 : Excellente Votre appréciation

Quelle note donneriez-vous à la qualité générale du coaching ?

\section{Qualité du coaching}

Échelle : 1 : Pas du tout d'accord - 2 : Un peu d'accord - 3: Assez d'accord - 4 D'accord -5 : Tout à fait d'accord

Les remontées d'informations (feedbacks) donnés par les coaches ont été utiles Les interventions des coaches ont été fondamentales dans l'élaboration des propositions d'amélioration de l'efficacité de la gestion

Les coaches ont renforcé mon habilité et ma confiance à prendre des décisions et à réagir sur des problèmes spécifiques de gestion de mon aire protégée

Les interventions des coaches ont amélioré l'esprit d'équipe et de collaboration entre le personnel de l'AP, et entre le personnel et les autres interlocuteurs et partenaires clés

La fréquence d'intervention des coaches était suffisante pour obtenir des résultats

Les coaches étaient disponibles pour tout supplément d'information ou demande d'aide

\section{Auto-évaluation de mes compétences}

\begin{tabular}{|l|l|}
\hline $\begin{array}{l}\text { Échelle : } 1: \text { Pas du tout capable - } 2: \text { Un peu capable - 3: Assez capable - } 4: \\
\text { Capable - 5: Très capable et confiant dans mes capacités }\end{array}$ & Votre appréciation \\
\hline À la suite de la mission de coaching, je suis capable de : & \\
\hline Expliquer et présenter sur & \\
\hline $\begin{array}{l}\text { Le fonctionnement général du Système d'information d'aide à la décision : } \\
\text { l'Observatoire régional des aires protégées et la biodiversité }\end{array}$ & \\
\hline Les avantages de l'utilisation du Système d'information & \\
\hline Expliquer les concepts clés du Formulaire BIOPAMA & \\
\hline La différence entre contexte de l'intervention et contexte de la gestion & \\
\hline Les menaces et les pressions sur les aires protégées et la biodiversité & \\
\hline Le cycle de gestion d'une aire protégée & \\
\hline
\end{tabular}




\begin{tabular}{|c|c|}
\hline \multicolumn{2}{|l|}{ Les méthodologies d'évaluation de l'efficacité de la gestion d'une aire protégée } \\
\hline La différence et la complémentarité entre gouvernance et gestion d'une AP & \\
\hline Les services écosystémiques & \\
\hline Remplir le Formulaire & \\
\hline Effectuer un pré-remplissage de la partie « Contexte de l'intervention » & \\
\hline $\begin{array}{l}\text { Remplir correctement la partie "Évaluation de l'efficacité de la gestion » : contexte de } \\
\text { la gestion, planification, intrants, processus, résultats, effets \& impacts }\end{array}$ & \\
\hline Analyser les résultats du Formulaire & \\
\hline $\begin{array}{l}\text { Formuler des propositions claires avec les valeurs de référence (benchmarks) dans le } \\
\text { Formulaire }\end{array}$ & \\
\hline Présenter les résultats & \\
\hline $\begin{array}{l}\text { Présenter l'analyse des résultats auprès des hiérarchies supérieures et partenaires } \\
\text { locaux }\end{array}$ & \\
\hline $\begin{array}{l}\text { Présenter et discuter des propositions d'améliorations concrètes au niveau des } \\
\text { hiérarchies supérieures et partenaires locaux }\end{array}$ & \\
\hline Mettre en œuvre les améliorations & \\
\hline $\begin{array}{l}\text { Mettre à jour mon plan de gestion ou plan de travail annuel en fonction des } \\
\text { propositions issues du Formulaire }\end{array}$ & \\
\hline Mobiliser mon équipe de gestion pour mettre en œuvre ces améliorations & \\
\hline Mobiliser des ressources matérielles et financières pour les projets d'améliorations & \\
\hline Pérenniser le système & \\
\hline Utiliser le Système d'information relayé par l'Observatoire des AP & \\
\hline Remplir le Formulaire l'année prochaine sans l'aide d'un coach & \\
\hline $\begin{array}{l}\text { Communiquer régulièrement et clairement les résultats de la gestion auprès des } \\
\text { partenaires }\end{array}$ & \\
\hline
\end{tabular}

\section{QUESTIONS ET REMARQUES SUPPLÉMENTAIRES}

Quelles sont vos suggestions pour renforcer vos capacités de gestion pour votre aire protégée à l'avenir?

Nous vous remercions d'avoir pris le temps de remplir ce formulaire.

\subsection{Ressources pour le coach}

\section{Fichiers électroniques}

0-4 C-EVAL1

0-5 C-EVAL2

0-6 C-EVAL3

\section{En cas de difficultés}

Évaluation des compétences et utilisation des formulaires

Domoina Rakotobe

domoina.rakotobe@ext.iucn.org 


\section{ANNEXE}

GLOSSAIRE

\begin{tabular}{|c|c|}
\hline Acteurs locaux & $\begin{array}{l}\text { Institutions, organisations, sociétés ou individus opérants dans le contexte } \\
\text { territorial d'intervention d'une AP. }\end{array}$ \\
\hline Aires protégées & $\begin{array}{l}\text { Territoires qui bénéficient d'un statut de conservation et qui, de ce fait, sont } \\
\text { l'objet d'une protection spéciale de la part des autorités gouvernementales. }\end{array}$ \\
\hline $\begin{array}{l}\text { Approche écosystémique } \\
\text { (approche par écosystème) }\end{array}$ & $\begin{array}{l}\text { Méthode de planification et/ou de gestion intégrant les terres, l'eau et les } \\
\text { ressources vivantes afin de favoriser leur conservation et leur utilisation durable } \\
\text { et de respecter leurs interactions dans des écosystèmes dont les hommes } \\
\text { dépendent. }\end{array}$ \\
\hline Biodiversité & $\begin{array}{l}\text { Désigne la diversité du monde vivant à tous les niveaux : diversité des milieux } \\
\text { (écosystèmes), diversité des espèces, diversité génétique au sein d'une même } \\
\text { espèce. Synonyme de diversité biologique. }\end{array}$ \\
\hline Bonne gouvernance & $\begin{array}{l}\text { Gestion transparente et responsable des ressources humaines, naturelles, } \\
\text { économiques et financières d'un pays ou d'une institution aux fins d'un } \\
\text { développement équitable et durable dans un environnement politique et } \\
\text { institutionnel respectueux des droits humains. Cette définition n'implique pas } \\
\text { une approche participative, mais relève plutôt d'une approche du haut vers le } \\
\text { bas. }\end{array}$ \\
\hline Cycle de la CMAP & $\begin{array}{l}\text { Enchaînement logique d'étapes de planification et d'intervention dans les AP, } \\
\text { recommandé par la Commission mondiale des aires protégées (CMAP) de } \\
\text { I'UICN. }\end{array}$ \\
\hline DDDAP & $\begin{array}{l}\text { Diminution des espaces de conservation, dégradation de la valeur et des } \\
\text { services écosystémiques, déclassement des Aires Protégées. Michael B } \\
\text { Mascia \& Sharon Pailler, World Wildlife Fund. }\end{array}$ \\
\hline Durabilité & $\begin{array}{l}\text { Par simplification, et dans le cas spécifique d'une gestion écosystémique, } \\
\text { la durabilité peut être assimilée à la résolution d'un puzzle complexe, qui } \\
\text { demanderait de rassembler et de relier harmonieusement, par un processus } \\
\text { adaptatif, les éléments relevant d'aspects écologiques, économico-financiers, } \\
\text { socio-territoriaux et politico-institutionnels et qui sous-tendent un système } \\
\text { d'interaction homme-nature. }\end{array}$ \\
\hline Écosystème & $\begin{array}{l}\text { Complexe dynamique, composé de plantes, d'animaux, de micro-organismes } \\
\text { et de la nature morte environnante agissant en interaction en tant qu'unite } \\
\text { fonctionnelle. Les éléments constituant un écosystème développent un résea } \\
\text { d'échange d'énergie et de matière permettant le maintien et le développemen } \\
\text { de la vie. }\end{array}$ \\
\hline Écosystème de référence & Écosystème dominant dans une aire protégée et dans sa zone contiguë. \\
\hline $\begin{array}{l}\text { Écosystèmes de référence } \\
\text { des AP }\left(^{*}\right)\end{array}$ & $\begin{array}{l}\text { Complexes territoriaux composés de zones sous différents régimes de } \\
\text { conservation ou de gestion des ressources naturelles (protection, exploitations } \\
\text { vivrières, artisanales, industrielles, etc.), régies par des juridictions diverses } \\
\text { (légale, légitime, d'interface, etc.) et des dispositifs multiples (cognitifs, } \\
\text { techniques, financiers, etc.). }\end{array}$ \\
\hline Environnement & $\begin{array}{l}\text { L'environnement désigne l'ensemble des conditions naturelles ou artificielles } \\
\text { (physiques, chimiques et biologiques) et culturelles (sociologiques) dans lequel } \\
\text { les organismes vivants se développent (dont l'homme, les espèces animales et } \\
\text { végétales). Le mot anglais environment signifie milieu. }\end{array}$ \\
\hline Gestior & $\begin{array}{l}\text { La gestion des AP est le processus par lequel les ressources humaines } \\
\text { et matérielles sont mises à profit pour atteindre un objectif connu dans une } \\
\text { structure institutionnelle donnée. }\end{array}$ \\
\hline
\end{tabular}




\begin{tabular}{|c|c|}
\hline $\begin{array}{l}\text { Gouvernance } \\
\text { environnementale }\end{array}$ & $\begin{array}{l}\text { "Conduite collective décisionnelle réunissant toutes les institutions et tous } \\
\text { les sujets qui sont dépositaires d'un pouvoir non-hiérarchique, ce qui impose } \\
\text { de mieux intégrer la planification et la gestion des ressources et de prendre } \\
\text { en considération les conséquences environnementales et sociales des } \\
\text { changements planifiés dans le temps". Selon cette définition, la gouvernance } \\
\text { environnementale respecte et renforce les principes de subsidiarité (sensu UE) } \\
\text { et les principes de l'approche participative des différentes parties prenantes } \\
\text { (approche du bas vers le haut). }\end{array}$ \\
\hline Instance de conservation & L'ensemble constitué des systèmes et des réseaux de conservation. \\
\hline Menaces & $\begin{array}{l}\text { Pressions, menaces et vulnérabilités actuelles et potentielles qui risquent d'avoir } \\
\text { un impact préjudiciable sur l'avenir, à court ou long terme, mais qui n'existent } \\
\text { pas encore aujourd'hui dans l'écosystème de référence (AP, périphérie ou } \\
\text { autre). }\end{array}$ \\
\hline Paysage & $\begin{array}{l}\text { Ensemble territorial constitué d'aires protégées, de zones de communautés de } \\
\text { base et de zones extractives - Programme CARPE. }\end{array}$ \\
\hline $\begin{array}{l}\text { Réseaux de coordination } \\
\text { de la conservation }\end{array}$ & $\begin{array}{l}\text { Ensemble des instances de conservation qui déterminent l'orientation, } \\
\text { l'organisation et l'intervention des systèmes de conservation selon les } \\
\text { dispositions au niveau local, national, transfrontalier, régional et global. }\end{array}$ \\
\hline $\begin{array}{l}\text { Services des (rendus } \\
\text { par les) écosystèmes ou } \\
\text { services écosystémiques } \\
\text { (source EM) }\end{array}$ & $\begin{array}{l}\text { Bienfaits que les humains obtiennent des écosystèmes. Ceux-ci comprennent } \\
\text { les services d'approvisionnement tels que la nourriture et l'eau, les services de } \\
\text { régulation tels que la régulation des inondations et des maladies, les services } \\
\text { culturels tels que les bénéfices spirituels, récréatifs et culturels, et les services } \\
\text { de soutien qui maintiennent des conditions favorables à la vie sur Terre, tels que } \\
\text { le cycle des éléments nutritifs. }\end{array}$ \\
\hline Subsidiarité & $\begin{array}{l}\text { Principe (sensu UE) visant à privilégier le niveau inférieur d'un pouvoir de } \\
\text { décision aussi longtemps que le niveau supérieur n'est pas capable d'agir plus } \\
\text { efficacement. }\end{array}$ \\
\hline $\begin{array}{l}\text { Système de conservation } \\
\text { (ou système protégé) }\end{array}$ & $\begin{array}{l}\text { Complexe territorial composé de zones soumises à différents régimes de } \\
\text { protection et/ou de gestion des ressources naturelles, par ex. : AP et sa } \\
\text { périphérie, parc et ses réserves de chasse voisines, AP et ses forêts contiguës, } \\
\text { AP et ses couloirs biologiques, etc. }\end{array}$ \\
\hline $\begin{array}{l}\text { Valeurs et importance des } \\
\text { aires protégées }\end{array}$ & $\begin{array}{l}\text { Les aires protégées sont gérées pour préserver les valeurs qu'elles renferment } \\
\text { et maintenir l'importance pour laquelle elles ont été classées. }\end{array}$ \\
\hline Vulnérabilités & $\begin{array}{l}\text { Aspects de faiblesse d'un système de conservation (par ex. épizooties } \\
\text { sporadiques, niveau élevé de pauvreté, etc.) qui, s'ils se déclenchent, risquent } \\
\text { d'avoir un impact préjudiciable sur le patrimoine et les ressources naturelles à } \\
\text { court ou long terme. }\end{array}$ \\
\hline
\end{tabular}







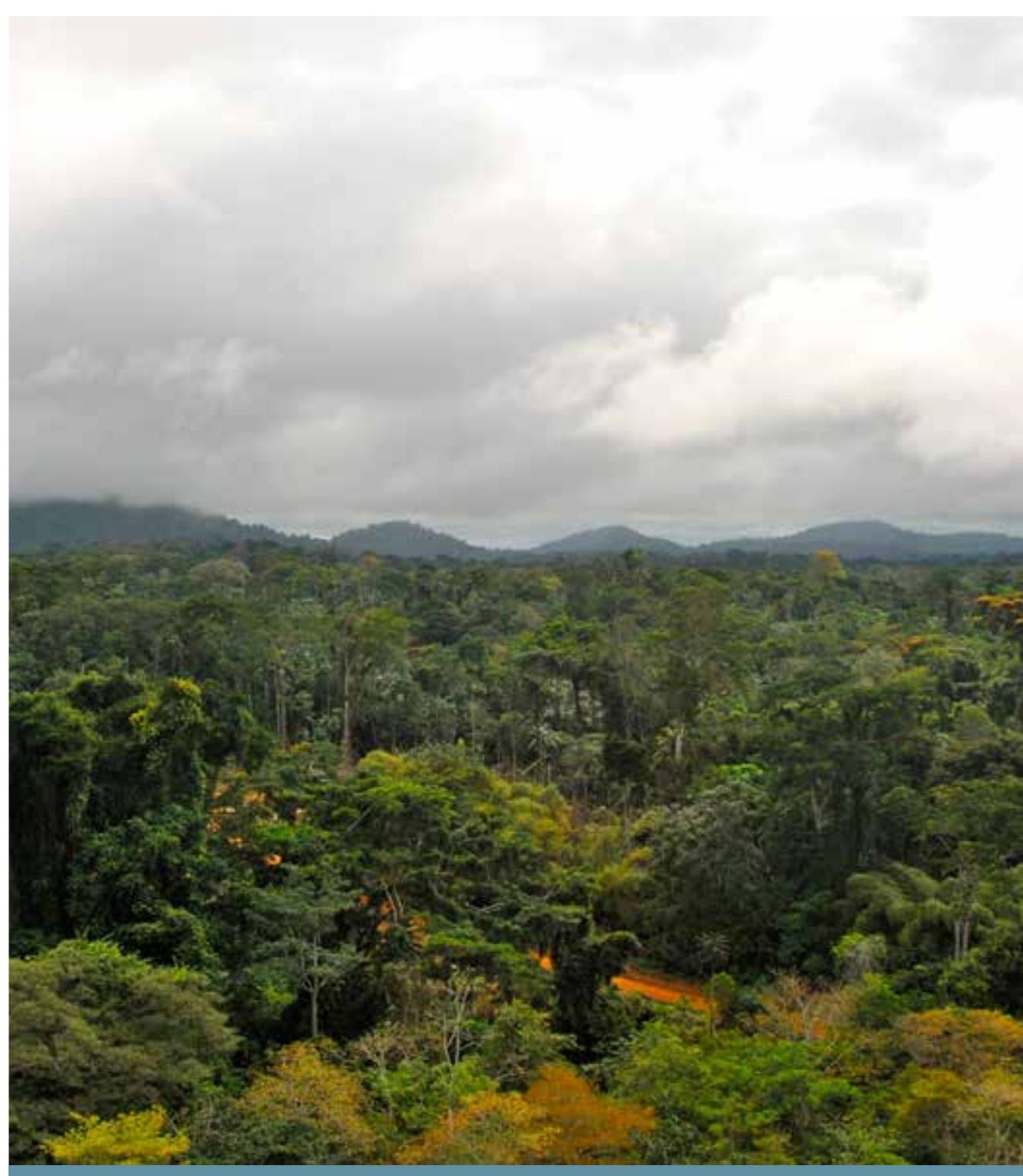

UNION INTERNATIONALE POUR LA CONSERVATION DE LA NATURE

BIOPAMA -

Le Programme pour la biodiversité et la

gestion des aires protégées

Rue Mauverney 28

1196 Gland, Suisse

biopama@iucn.org

www.biopama.org 